\title{
The Mechanical Response of a Hybrid Foam Glass-Epoxy Composite Corrugated Cellular Structure Sandwich Panel
}

\author{
A Dissertation \\ Presented to \\ the faculty of the School of Engineering and Applied Science \\ University of Virginia \\ in partial fulfillment \\ of the requirements for the degree of
}

Doctor of Philosophy

Mechanical and Aerospace Engineering

by

Adam J. Malcom

August

2014 


\section{Approval Sheet}

The dissertation

is submitted in partial fulfillment of the requirements

for the degree of

Doctor of Philosophy

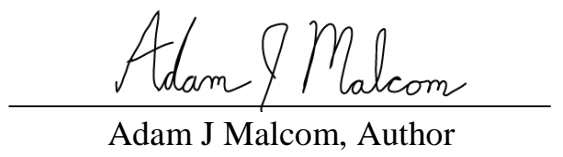

This dissertation has been read and approved by the examining committee:

\begin{tabular}{c} 
Pamela Norris \\
\hline Advisor \\
Mark Aronson \\
\hline Advisor \\
Dana Elzey \\
\hline Committee Chair \\
Hilary Bart-Smith \\
\hline Jim Howe \\
\\
Robert Kelly \\
\\
Richard Kent
\end{tabular}

Accepted for the School of Engineering and Applied Science:

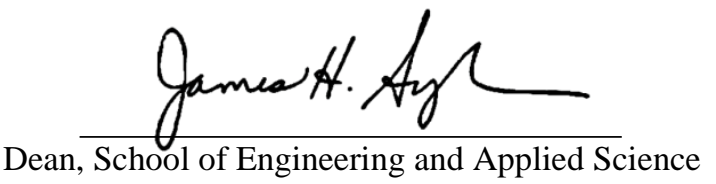

August

2014 


\section{Abstract}

The mechanical response of a novel hybrid glass-fiber composite corrugated cellular structure sandwich panel when subjected to out-of-plane compressive loading is investigated to evaluate the structure's potential adaptation into vehicle armor. Inspired by the persistent military need to develop effective, lightweight armor for vehicle protection against buried Improvised Explosive Devices (IEDs), this study provides a detailed analysis of the compressive response of the hybrid corrugated sandwich structure under quasi-static and dynamic loading. The through-thickness (out-of-plane) compressive strength, stiffness, densification strain, and energy absorption of the core is measured through quasi-static loading, along with peak failure strengths and impulse mitigation under dynamic compression and high explosive sand blast loading. The goal is to identify and characterize the quasi-static response of this novel hybrid composite corrugated sandwich panel core, core struts, and associated constitutive materials through empirical testing, develop quasi-static analytic predictions for the strength and modulus of the core and core struts, and finally, empirically investigate the dynamic strength and impulse mitigation performance along with the associated dynamic failure mechanisms. A vehicle armor must be able to provide through-structure pressure reduction and impulse mitigation.

The hybrid composite corrugated sandwich panel concept is the first all-inclusive, singlestep infused composite sandwich panel ever to be manufactured. Constructed using a delamination resistant, three dimensionally (3D) woven fiber architecture, E-glass is used to construct a corrugated core with struts oriented at $60^{\circ}$ (from the horizontal) and a stronger S2glass is used for the facesheets. Divinycell H130 PVC foam is used to support the fiber structure in the corrugated pattern during infusion and provide a foam-strut stabilization to increase the compressive strength. A modified-Vacuum Assisted Resin Transfer Molding (VARTM) process was used in conjunction with a novel pressure differential technique created to construct core struts with fiber volume fraction ranging from 30-60\% . Compressive strut failure was observed to be governed by Euler buckling at low strut slenderness ratios $(\mathrm{t} / 1 \leq 0.07)$ and completely transitioned to plastic microbuckling at higher ratios $(\mathrm{t} / \mathrm{l} \geq 0.14)$. For low fiber volume fraction struts, $v_{\mathrm{f}} \approx 35 \%$, strengths ranged from $85-125 \mathrm{MPa}$ while for high fiber fraction struts, $\mathrm{v}_{\mathrm{f}} \approx 56 \%$, strengths ranged from 100-275 $\mathrm{MPa}$. The elastic modulus relation was approximately linear to 
the fiber volume fraction ranging from 12-25 GPa. Negligible differences in the strength and modulus of the E-glass and S2-glass composite struts under compressive loading were observed, revealing a significant factor in material choice for glass based composites used in compressive design. Struts failing by plastic microbuckling were hypothesized to be largely influenced by the matrix shear strength and the initial average fiber tow misalignment angles rather than fiber properties. Therefore, the 3D woven fiber architecture, which imparted significant fiber waviness and misalignment angles to the axis of loading, was characterized using high resolution X-Ray Computed Tomography and optical microscopy, revealing an average misalignment angle of $1.5^{\circ}$ and $1.8^{\circ}$ in for the E-glass fabric and $1.6^{\circ}$ and $1.5^{\circ}$ in the S2-glass for the weft and warp fiber tows respectively. Localized peak tow misalignment angles of $11.2^{\circ}$ and $22.4^{\circ}$ were also measured for E-glass and S2-glass, respectively. Model predictions were created and indicate initial average misalignment range from $1.5^{\circ}$ to $5^{\circ}$ for all struts tested, indicating failure is due to the averaged misalignments angles and not a localized peak misalignment of the fiber tows throughout the strut. Model predictions were developed to provide first-order prediction of the compressive strength and modulus of a 3D woven composite. These predictions provide better agreement than first-order models previously developed and presented by outside researchers.

The quasi-static compressive stress-strain response of the panels was experimentally investigated as a function of the strut slenderness ratio $(\mathrm{t} / \mathrm{l})$, fiber volume fraction, relative density, and core fill (i.e. foam-filled versus open core). Cores with relative densities ranging from $15-45 \%$ were constructed by varying the strut slenderness ratios from $0.07<\mathrm{t} / 1<0.25$ providing cores with densities ranging from $280-775 \mathrm{~kg} / \mathrm{m}^{3}(3.5-10 \%$ the density of steel) providing a lightweight, high strength structure. Open core strengths were governed by Euler buckling of the struts for low slenderness ratios $(\mathrm{t} / 1 \leq 0.07)$ and strut plastic microbuckling $(\mathrm{t} / 1 \geq$ 0.14), confirming predictions of the strut analysis. All foam-filled cores failed by plastic microbuckling indicating a stabilization effect of the foam when failure occurred by Euler buckling. Average open core compressive strengths ranged from 5-33 MPa while foam-filled cores ranged 12-36 MPa. Strength differences between open and foam-filled cores that fail by plastic microbuckling indicate an approximate $3 \mathrm{MPa}$ strength increase, equivalent to the compressive strength of the foam inserts indicating no synergism. A strength increase of 6-7 MPa was observed for the samples failing by Euler buckling, indicating that the foam provides strut stiffening and a synergistic effect on compressive strength. 
Micromechanical models are developed to predict the compressive strength, modulus, densification strain, and energy absorption of the core. Strength models use experimentally measured Euler and plastic microbuckling strengths and successfully predict core strength. The compressive modulus prediction uses a small strain, pin-jointed strain analysis of the core struts and closes matches the idealized loaded structures, but poorly predicts structures with inherent imperfections that create unequal strut loading. A new densification strain model is developed to reflect the mechanical "hinge" response observed in the strut during loading and exhibits good agreement between the model and experiments, with densification strains ranging from 53-62\% and 40-44\% for open and foam-filled cores, respectively. Finally, for comparison purposes, the hybrid corrugated sandwich panel response is compared with classic stochastic foam models to illuminate the change in response of this structure from a purely stochastic material.

The dynamic out-of-plane compressive response of the foam-filled and open core corrugated sandwich panels was measured for impact velocities up to $175 \mathrm{~ms}^{-1}$ of a cylindrical projectile when fired from a ballistic gas gun. Stresses on the rear faces of the samples were measured using a direct impact Kolsky Bar showing dynamic peak strengths plateauing at around $100 \mathrm{MPa}$, and transition of failure from plastic-microbuckling to fiber crushing. The foam inserts provided a negligible beneficial effect on the measured response. This result illuminates the potential upper limit for peak strength of this structure at high strain rates.

Finally, high-explosive blast loading was used to evaluate the response under high velocity impacts at sand velocities up to $400 \mathrm{~ms}^{-1}$ on multi-tier corrugated sandwich panels. Through the use of a novel vertical pendulum rig designed for this experiment, an impulse mitigation of up to $30 \%$ is observed for this structure, indicating the existence of a critical impulse mitigation regime. Rear-face pressure measurements are measured through the unique conversion of the sliding bars of the vertical pendulum into a series of fixed Kolsky Bars. Maximum rear-face pressure reductions of 30-40\% were observed in the structure as compared to a rigid (aluminum) material comparison. The results show a light-weight structure that is capable of significant impulse mitigation and pressure reduction under specific loading parameters. Combined with this structures unique ability to be easily redesigned, optimized, and manufactured to meet alternate loading scenarios, the continued evaluation as a vehicle blast protection armor under real-world threat criteria is warranted. 
Nothing in life is to be feared, it is only to be understood. Now is the time to understand more, so that we may fear less.

Madame Marie Curie

Any intelligent fool can make things bigger, more complex, and more violent. It takes a touch of genius -- and a lot of courage -- to move in the opposite direction.

Albert Einstein

I've learned that people will forget what you said, people will forget what you did, but people will never forget how you made them feel.

Maya Angelou 


\section{Acknowledgements}

My progress and education successes were the result of continuous encouragement, support, and motivation from so many people, particularly my family, friends, and colleagues. Their support was critical to understanding the technical aspects of this project, participating in new and exciting research opportunities, and finding the inspiration and motivation to push forward when times were tough. The greatest lessons and most useful help came from the opportunities I've been given to work alongside some of the finest people I've met in my life. The compliments, criticisms, discussions, and adventures have proven to be the most invaluable aspect of my time spent at UVA. I wish to express my deepest and most sincere gratitude to everyone who supported me throughout my research.

I would like to express my gratitude to all of my advisors, Mark Aronson, Haydn Wadley, and Pam Norris, for their help, support, and challenges throughout this research. Each of you made a sacrifice to help me succeed and your efforts have made every step of this journey a lesson in life. I deeply thank Mark Aronson for his consistent guidance and confidence in this research and my abilities. I sincerely thank Haydn Wadley for his time, effort, and resources that he invested in both me and this research project. I wholeheartedly thank Pam Norris for her willingness to listen, help, and make my education a success. I would also like to also thank my committee members, Hilary Bart-Smith, Dana Elzey, James Howe, Robert Kelly, and Richard Kent for their time, energy, patience, and willingness to help me reach my goals.

I additionally would like to thank the following people for their instrumental efforts and support:

- To Ryan Holloman, Mark O’Masta, Scott Kasen, Toni Kember, and J.J. Wetzel for their support in the "trenches". Thanks for looking out for each other along the way. 
- To Kumar Dharmasena for being the most patient man I have ever met in my life.

Don't ever underestimate your importance to those around you.

- To Lewis Steva and Richard White, I learned more working in your labs and workshops under your guidance than in any classroom.

- To James Fitz-Gerald, Bill Johnson, and Kim Fitzhugh-Higgins for caring about the well-being of a student struggling to stay afloat.

- To Sherri Sullivan and Tommy Eanes for their help and support throughout this project.

- To Arve Hanssen, Wayne Mindle, and Lars Olovsson for their help with my modeling efforts with IMPETUS. Modeling with IMPETUS was a highlight in my research.

- To Vikram Deshpande and Benjamin Russell for the opportunity to collaborate on dynamic testing and your assistance throughout the analysis of this structure.

- To Keith Williams, Mike Lipscomb, and James White, along with the resources of Force Protection., General Dynamics, and NEWTEC for providing the ability and environment to perform high explosive blast testing.

- To Dawn Brotherton, Tom McCormick, and Bert Hawkins for helping balance the demands of school and work.

- To Tannis Fuller, for being the sound of reason and such a good friend.

Most importantly, I thank my family for their unyielding love, motivation, and confidence. I am continually amazed at the absolute confidence you have in my abilities and unconditional love you have shown to me all of my life. I am blessed to be so fortunate to have your support every day of my life. 


\section{Funding Source}

This research was funded through the Office of Naval Research (ONR) under grant number N00014-07-1-0764 (Program manager, Dr. David Shifler). There is much gratitude for this support. 


\section{Table of Contents}

APPROVAL SHEET I

$\begin{array}{lll}\text { ABSTRACT } & \text { II }\end{array}$

ACKNOWLEDGEMENTS VI

FUNDING SOURCE VII

TABLE OF CONTENTS IX

LIST OF FIGURES XII

LIST OF TABLES TXIV

LIST OF SYMBOLS IXV

CHAPTER 1 INTRODUCTION 1

1.1 Rationale for the Study 1

1.2 Hybrid Corrugated Sandwich Panel - Preliminary Blast Loading 6

1.3 Dissertation Goals and Outline 15

CHAPTER 2 HIGH PERFORMANCE COMPOSITE MATERIALS 19

2.1 High Performance Materials 19

2.1.1 Fibers 21

2.1.2 Matrix Materials 27

2.1.3 Manufacture Methods of Fiber Reinforced Polymer (FRP) Composites 29

CHAPTER 3 STRUCTURAL SYSTEMS 33

3.1 Cellular Structures $\quad 34$

3.2 Cellular Structure Failure Mechanisms 38

3.2.1 Bending-Dominated Structures 38

3.2.2 Stretch-Dominated Structures

3.2 Sandwich Panel Structures 44

3.3 High Explosive Blast on Sandwich Panel Designs 48

CHAPTER 4 STRUCTURE DESIGN AND MATERIAL SELECTION 51

4.1 Hybrid Corrugated Sandwich Panel Design $\quad 52$

4.2 Material Selection and Properties $\quad 55$

4.2.1 E-Glass Fiber $\quad 55$

4.2.2 S2-Glass Fiber $\quad 56$

4.2.3 Kevlar $^{\circledR}$ Fiber $\quad 57$

4.2.4 SC-11 Epoxy 59

4.2.5 Divinycell H130 Foam $\quad 62$ 
4.3 3D Woven Fiber Architecture $\quad 63$

$\begin{array}{ll}4.4 \text { Relative Density } & 67\end{array}$

CHAPTER 5 FABRICATION METHODOLOGY 69

5.1 Dry Fabric Hybrid Structure Manufacture $\quad 70$

5.2 Vacuum Infusion Process

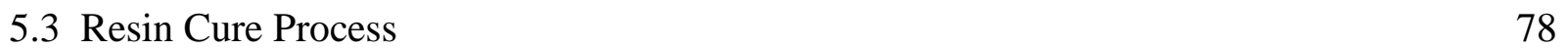

5.4 Post-Cure Panel Fabrication $\quad 81$

5.5 Manufacturing Imperfections $\quad 83$

CHAPTER 6 3D WOVEN COMPOSITE STRUT QUASI-STATIC LOADING 87

6.1 Characterization $\quad 89$

6.1.1 X-Ray Computed Tomography $\quad 91$

6.1.2 Optical Microscopy 99

$\begin{array}{ll}6.2 \text { Tension } & 103\end{array}$

6.2.1 Test Methodology- Strut Tension 103

6.2.2 Experimental Results-Strut Tension 104

6.2.3 Analytical Predictions-Strut Tension 106

6.3 Compression 109

6.3.1 Test Methodology- Strut Compression 109

6.3.2 Experimental Results - Strut Compression 110

6.3.3 Analytical Predictions - Strut Compression 116

CHAPTER 7 HYBRID CORRUGATED CORE SANDWICH PANEL QUASI-STATIC COMPRESSION $\quad 123$

7.1 Single-Tier Sandwich Panel 123

7.1.1 Test Methodology - Single-Tier 123

7.1.2 Experimental Results - Single-Tier 124

7.1.3 Analytical Predictions - Single-Tier 138

7.2 Multi-Tier Sandwich Panel 146

7.2.1 Test Methodology - Multi-Tier 146

7.2.2 Experimental Results - Multi-Tier 147

7.3 Comparison with a Foam Material Response 152

CHAPTER 8 HYBRID CORRUGATED SANDWICH PANEL DYNAMIC COMPRESSION AND HIGH-EXPLOSIVE BLAST LOADING 158

8.1 Dynamic Compressive Loading 158

$\begin{array}{ll}\text { 8.1.1 Test Methodology } & 158\end{array}$ 
8.1.1 Experimental Results - Strut 163

8.1.2 Experimental Results - Single-Tier 165

8.2 High-Explosive Compressive Blast Loading 169

8.2.1 Test Methodology - High Explosive Blast Loading - Multi-Tier 170

8.2.2 Experimental Results - High Explosive Blast Loading - Multi-Tier 183

CHAPTER 9 RESEARCH CONTRIBUTIONS AND CONCLUSIONS 206

$\begin{array}{ll}9.1 \text { Contributions } & 207\end{array}$

9.2 Conclusion - Quasi-Static Response 210

9.3 Conclusion - Dynamic Response 213

CHAPTER 10 FUTURE RECOMMENDATION AND RESEARCH EVALUATION 216

10.1 Finite Element Modeling 216

10.2 Potential Improvements 218

10.3 Overall Research Assessment 220

$\begin{array}{ll}\text { REFERENCE } & 222\end{array}$ 


\section{List of Figures}

Figure 1: Explosive devices have been used by terrorist to damage both sea and land vehicles such as a) the USS Cole [6] and b) the Cougar MRAP vehicle [7] ........................... 3

Figure 2: Common boundary conditions associated with explosive blast testing include a) edgeclamped conditions allowing for panel stretching and deformation and b) rear-face supported conditions used to evaluate panel crushing...........................................

Figure 3: The substation of a portion of a foam core sandwich structure with a periodic corrugated cellular structure resulted in the hybrid foam glass-epoxy composite corrugated sandwich panel concept developed for analysis as an armor technology... 8

Figure 4: Edge clamped corrugated core blast panel ...................................................... 9

Figure 5: Hybrid foam glass-epoxy composite corrugated sandwich panel manufactured for an edge-clamp blast loading scenario. a) A top-down view of the untested panel shows the core and integrated mounting frame. b) The panel as mounted for the edgeclamped explosive sand-blast test.

Figure 6: Edge-clamped loading scenario of the hybrid foam glass-epoxy corrugated composite sandwich panel. a) The explosive charge is created with a spherical 150 gram charge of $\mathrm{C} 4$ explosive surrounded by a $150 \mathrm{~mm}$ diameter sphere comprised of glass microspheres saturated in water. b) The sample is rigidly mounted with the explosive charge/sand ball suspended above the panel with a distance of $15 \mathrm{~cm}$ from the charge center to the panel front face. 10

Figure 7: Normalized rear-face deflections $\left(\delta / L_{p}\right)$ measured for the hybrid composite sandwich panel and equivalent mass laminated composite and aluminum panels under three different impulse loads corresponding to a bare, dry, and wet charge type all using 150 grams of $\mathrm{C} 4$ explosive.

Figure 8: Post-blast images of the hybrid panel tested in an edge-clamped scenario with a 150 gram C4 explosive charge encapsulated in a water saturated glass microsphere soil layer. Observed failure included: a) and c) S2-glass facesheet tensile failure and burning in sample center and front facesheet shear along core/frame boundary, b) Rear facesheet shear and matrix crazing at positions of core displacement, and d) nodal failure exhibiting stitch and matrix failure along with foam core/facesheet bond failure.

Figure 9: Analysis of the failure mechanisms of the edge-clamped test samples show that that the boundary conditions created a "point-of-contact" variable between the facesheets and either the aluminum mounting frame or permanent rig. Shear failure was prematurely induced by sharp edges that could transmit large shear loads to the facesheets. 
Figure 10: Shear failure was observed in the nodal stitching and Divinycell foam core resulting from the large deformations of the hybrid composite sandwich structure. 15

Figure 11: Strength plotted against density for structural materials (yield strength is plotted for metals and polymers, compressive strength for ceramics, and tensile strength for composites) [21]

Figure 12: Young's modulus, E, plotted against failure strength [21].

Figure 13: Specific tensile strength versus specific modulus for select metals, polymers, and composite fibers.

Figure 14: A comparison of yarn-to-fabric preforms including weave, braid, and knitting processes [24]. 25

Figure 15: Variations in a biaxial 2D woven fabric [23] 26

Figure 16: Comparison of a woven roving and knitted biaxial fiber architecture illustrate the fiber waviness/misalignment that is inherent to a given fiber architecture design [23].

Figure 17: Usable temperature ranges for composites with different matrix materials [22]...... 28

Figure 18: Variations in the elastic modulus of polymers with temperature: (a) thermoplastic amorphous, (b) thermoset amorphous, (c) thermoplastic semicrystalline [22].

Figure 19: Examples of cellular structures morphology to include (a) open-cell and (b) closed cell structures. Both structures represent a stochastic cellular structure [30]. 34

Figure 20: Stretch-dominated (a) unit cell and (b) lattice structure [30]. 36

Figure 21: Bending-dominated structures fail by slightly different methods depending upon the material. (a) Ductile material causes cell edge bending. (b) Elastomeric materials cause cell edge buckling. (c) Brittle materials collapses by successive fracturing of cell edges $[26,28,30]$.

Figure 22: Classic stress-strain response of a bending-dominated cellular structure including both open and closed-cell stochastic foams. 40

Figure 23: A stretch-dominated structure is stiff when stressed because loads are transmitted axially along the ligaments. For comparisons a diamond cell is (a) unloaded, (b) loaded without a transverse stiffener, and (c) loaded with a transverse stiffener to create a stretch-dominated structure [30].

Figure 24: Classic stress-strain response of a stretch-dominated cellular structure. 
Figure 25: A sandwich panel structure is created by attaching a core (such as a honeycomb cellular structure as shown) to facesheet [62].

Figure 26: Examples of sandwich panel structures with various core topologies $[28,52,59] \ldots 46$

Figure 27: Design parameters associated with the hybrid foam glass-epoxy corrugated composite celluar structure sandwich panel.

Figure 28: Measured tensile stress-strain responses of E and S2-glass fiber tows removed from the 3D woven fiber architectures compared to the theoretical response of ideal silane coated fibers used in this fabric.

Figure 29: A representative tensile stress measurement for the Kevlar thread and compared to virgin fiber tensile tenacity at strain failure [103]. 58

Figure 30: Compressive and shear stress-strain response for the rubber toughened SC-11 epoxy resin used as the matrix in the hybrid composite structure.

Figure 31: The loss tangent $(\tan \delta)$ is plotted as a function of temperature to reveal the glass transformation temperature of the SC-11 epoxy resin. SC-1 and vinyl ester are shown for comparison.

Figure 32: The elastic modulus of SC-11 epoxy is plotted as a function of temperature. SC-1 and vinyl ester resins are plotted for comparison.

Figure 33: Compressive stress-strain response of H130 Divinycell foam.

Figure 34: Fiber architecture of the 3D woven glass fiber fabrics used in the core struts and face sheets of the corrugated core sandwich structure. The 3D woven geometry consists of three weft tows, two warp tows and one z-yarn per repeating volume element. 65

Figure 35: Photograph of the (a) E-glass 3D woven fabric (3Tex 3 Weave ${ }^{\circledR}$ fabric) used for the core struts and (b) the S2-glass $3 \mathrm{Weave}^{\circledR}$ used for the face sheets. The E-glass 3 Weave ${ }^{\circledR}$ fabric had a larger $z$-yarn spacing resulting in a looser weave which facilitated fabric folding to form a core corrugation.

Figure 36: Schematic illustration of the assembly process of the hybrid corrugated sandwich panel.

Figure 37: A locking straight stitch, commonly called a lockstitch, was used for nodal core-tofacesheet connections.

Figure 38: Basic layout of the Vacuum Infusion Process (VIP) used for resin infiltration of the composite structure. 
Figure 39: A slightly modified Vacuum Assisted Resin Transfer Mold (VARTM) technique uses a vacuum bag sealed to a rigid glass substrate to create a vacuum cavity for the part infusion. 75

Figure 40: The vacuum infusion process of the multi-tier structure utilized a resin inlet line positioned at the top of the part and a vacuum line positioned $360^{\circ}$ around the base of the part. 77

Figure 41: Econoclave EC3X5 composite autoclave was used to provide the necessary temperature and pressure for resin cure. The modified VARTM process occurs inside the autoclave at ambient temperature and pressure, as shown. 78

Figure 42: Illustration of the temperature and pressure cure process of the autoclave and vacuum bagged part. 80

Figure 43: Cured composite structures are precisely cut into the desired shape for quasi-static or dynamic testing. The two-cell structures were used for laboratory dynamic impact testing.

Figure 44: The multi-tier panel created for high explosive blast testing. 82

Figure 45: a) Photograph of an as-manufactured panel (Design 3) and b) schematic illustration of node height variations resulting from stitch offset during manufacture. Imperfections resulted in small variations of strut length, 1 , and strut orientation angle, $\omega$.

Figure 46: Unit cell manufactured with epoxy filler on the facesheets to minimize imperfections and uniformly distribute pressure. 85

Figure 47: Manufacturing limitations create nodal misalignments throughout the multi-tier structure. These misalignments create moments around the node during loading and can alter the failure mode from a stretch-dominated design to bending-dominated... 86

Figure 48: Schematic illustration of a hybrid polymer foam/corrugated composite core sandwich panel utilizing 3D woven E-glass fiber textile to create the core struts and S2-glass for the face sheets. When subjected to bending, the face sheets and core struts are loaded in either in-plane tension or compression. 88

Figure 49: Kink bands are formed under axial compressive loading of fibers where a group of fibers locally bends along a plane inclined at angle $\beta$. Drastically influenced by the initial fiber misalignment angle, $\phi$, failure can result in the formation of plastic kink bands or even fractured kink bands. [114]. 
Figure 50: XCT images of a single laminate E-glass strut. (a) The 3D structure illustrates maximum fiber waviness at the point the z-yarn tows contacts the weft fiber tows. Cross sectional slices show the (b) warp and (c) weft fiber tows respectively. The dotted lines illustrate the epoxy surface location and the vertical bands A and B indicate planes for which tow misalignment data is presented. This structure is void free and internal void areas are filled with epoxy.

Figure 51: Initial fiber tow misalignment angles are measured for a single laminate E-glass composite strut. Warp fiber tow misalignment angles are provided for positions (a) adjacent to a "mid-weft" tow (Position A, Figure 4b) and (b) furthest position away from the weft tow (Position B, Figure 4b). Weft fiber tow misalignment angles are provided for positions (c) adjacent to the z-yarn (Position A, Figure 4c) and (d) "midwarp" tow position (Position B, Figure 4c). These positions provide the relative extremes observed in the fiber waviness along the length of the sample. 94

Figure 52: XCT images of a 2 and 3 laminate E-glass strut illustrate similar fiber waviness as a single laminate E-glass strut. Cross sectional slices show the warp and weft fiber. This structure is void free and internal void areas are filled with epoxy. 96

Figure 53: XCT images of a single laminate S2-glass strut. (a) The 3D structure illustrates maximum warp and weft fiber tow waviness near the point where the z-yarn tow contacts the weft fiber tows. Cross sectional slices containing the (b) warp and (c) weft fiber directions. The dotted lines illustrate the epoxy surface location and the vertical bands A and B indicate planes for which tow misalignment data is presented. This structure is void free and internal void areas are filled with epoxy. 97

Figure 54: Initial fiber tow misalignment angles are measured for a single laminate S2-glass composite strut. Warp fiber tow misalignment angles are provided for positions (a) adjacent to a "mid-weft" tow (Position A, Figure 7b) and (b) furthest position away from the weft tow (Position B, Figure 7b). Weft fiber tow misalignment angles are provided for positions (c) adjacent to the z-yarn (Position A, Figure 7c) and (d) "midwarp" tow position (Position B, Figure 7c). These positions provide the relative extremes observed in the fiber waviness along the length of the sample.

Figure 55: Optical micrographs of the E-glass $3 \mathrm{Weave}{ }^{\circledR}$ composite. Both the horizontal warp tows (a) and horizontal weft tows (b) exhibit waviness resulting from out of plane zyarn compression. (c) Weft tow misalignment was greatest at the position of z-yarn impingement. (d) The diameter of the E-glass fibers was $18 \mu \mathrm{m} \pm 4 \mu \mathrm{m}$ for the approximately 2,300 fibers within a weft tow. 101

Figure 56: Low magnification of optical micrographs of the S2-glass 3 Weave ${ }^{\circledR}$ composite illustrate the (a) z-yarn square weave binding the weft fiber tows, (b) the misalignment of the horizontal weft tows resulting from the localized pressure applied by the binding z-yarn, (c) weft tow misalignment near a z-yarn contact point, and (d) the diameter of the $\mathrm{S} 2$-glass fibers was $9 \mu \mathrm{m} \pm 1 \mu \mathrm{m}$ for the approximately 8,000 fibers within a weft tow. 
Figure 57: Tensile test coupons of the 3D woven composite. a) Samples where cut to length and bonded to gripping tabs to prohibit stress concentrations. b) The specimen was clamped in wedge action compression grips with the weft fiber direction oriented parallel to the axis of loading.

Figure 58: The fiber volume fraction dependence of the tensile modulus for E and S2-glass composite struts. Predictions use the experimentally measured modulus of the postwoven silane coated fibers which closely matches reported virgin fiber properties. 105

Figure 59: The fiber volume fraction dependence of the tensile strength for the E and S2-glass composites. Model predictions, Equation 6.4,are bounded using the theoretical strength of a silane coated fiber and the experimentally measured strength of the postwoven fiber tow rovings. 106

Figure 60: The Combined Load Compression (CLC) rig was used for confinement of the composite samples. a) The test specimen was cut to length and clamped in the CLC fixture. b) The weft fiber tows were aligned parallel the axis of loading. 110

Figure 61: (a) Compressive stress-strain response of the E-glass struts. (b) and (c) are examples of compressive fiber microbuckling and (d) Euler elastic buckling failure of E-glass struts loaded parallel to the weft tow direction with thickness to length ratios of (b) $0.18(5.25 \mathrm{~mm}),(\mathrm{c}) 0.12(3.5 \mathrm{~mm})$, and (d) $0.07(1.75 \mathrm{~mm})$.

Figure 62: Measured and predicted Young's modulus verses fiber volume for E and S2-glass composite struts tested in compression parallel to the weft fiber tows. Fiber waviness has been shown by Cox et al. [109] to reduce the elastic modulus by up to $40 \%$ in 3D woven composites. It is hypothesized that misalignment is the predominant factor for the data scatter of this experiment.

Figure 63: Measured and predicted compressive strength for E and S2 glass composite struts loaded parallel to the weft fiber tows. At low ratios of $t / 1<0.07$ the struts failed by Euler (elastic) buckling. As $\mathrm{t} / \mathrm{l}$ increased, strut failure occurred by fiber microbuckling.

Figure 64: Dependence of compressive strength upon fiber volume fraction for E and S2 glass composite struts with $\mathrm{t} / \mathrm{l}>0.07$ loaded parallel to the weft fiber tows. Failure of weft tow laminates is dependent upon the fiber misalignment angle, $\phi$, but independent of the fiber strength, while failure of the warp tow laminates is dependent upon the fiber strength but independent of the fiber misalignment angle. 114

Figure 65: (a) Optical micrographs of an E-glass strut that failed by microbuckling under compressive load. (b) Fiber fracture in weft tows and matrix shear in warp tows accompany the double kink microbuckling mechanism. 
Figure 66: Illustration of the iso-strain loading diagram for the micromechanical model of a single laminate 3D woven strut. The micromechanical model assumes including equal tow spacing, equal tow sizes, and the absence of the z-yarn.

Figure 67: The compression fixture for the hybrid sandwich panel provides rear face and lateral boundary condition support during testing.

Figure 68: Compressive stress-strain response for the multi-cell glass-epoxy corrugated sandwich structures for the a) Divinycell H130 foam-filled structure and b) open core structure.

Figure 69: Compressive stress-strain response for unit-cell glass-epoxy corrugated sandwich panel structures for the a) Divinycell H130 foam-filled structure and b) open core structure.

Figure 70: Photographs taken during compression of the foam-filled "Design 3" sandwich structure. Initial failure of the trusses occurs by micro-buckling of the struts. Parts a-f correspond to the multi-core structure and parts g-l correspond to the single, unit-cell structure for strains of $0 \%, 10 \%, 20 \%, 30 \%, 40 \%$, and $50 \%$ respectively. The "white" regions correspond to matrix crazing. 128

Figure 71: Photographs taken during compression of the open core "Design 3" structure. Initial failure of the trusses occurred by micro-buckling of the struts. Parts a-f correspond to the multi-core structure and parts g-l correspond to the single, unit-cell structure for strains of $0 \%, 10 \%, 20 \%, 30 \%, 40 \%$, and $50 \%$ respectively. The "white" regions correspond to matrix crazing.

Figure 72: The (a) composite corrugated sandwich structure exhibited many failure modes (b to g) during the compression testing up to the densification limit. Predominant failure was initiated by (b) elastic buckling, (c) unfractured double kinking of the fiber tows or (d) fractured fiber kinking of the fiber tows. Subsequent shear fractures were observed after fiber kinking occurred.

Figure 73: Elastic modulus data and micromechanical predictions for the foam-filled and open core structures as a function of relative density. Imperfections in multi-cell samples resulted in non-uniform strut loading and a measured modulus significantly lower than model predictions. The measured modulus of the single unit-cell samples are in good agreement with the micromechanical model predictions representative of the fiber volume fractions measured in the core struts $\left(30 \%<v_{\mathrm{f}}<40 \%\right)$.

Figure 74: Peak compressive strength measurements and predictions for the corrugated composite core. a) Divinycell H130 foam-filled core and b) the open core. Results are shown as a function of the calculated relative density of the open core structure only. The predicted strengths of the corrugated structures are based on the experimentally measured critical strengths of the strut, Table 8 . The range and average measured strength is shown 
Figure 75: The specific strength versus the specific modulus for the hybrid composite corrugated sandwich panel is compared with several metals and polymers. The hybrid structure provides a higher specific strength than common structural steel and cast iron, but does not match the performance of aluminum 6061 or stainless steel 304L, previously studies for blast applications. 133

Figure 76: Densification strain measurements are shown for the unit cell structures (open and foam-filled) and various grades of Divinycell foam. The densification strain data for the foams is closely approximated by a linear model proposed by Maiti et al. [58]. Core predictions are based on the developed strut hinge model using specific parameters unique to each core geometry (green markers). The strut hinge densification model is simplified using fixed constants $x=0.70$ and $\omega=60^{\circ}$ (solid black line) with anticipated trends illustrated to the bounding limits (dotted lines). 135

Figure 77: The energy absorbed per unit volume during compression to the densification strain. Data is shown for the empty and foam-filled corrugated core composite structures investigated here and compared to Divinycell foams. Energy absorption predictions (based on the micromechanical models developed in this study) are illustrated for comparison with experimental data.

Figure 78: The energy absorbed per unit mass of the composite corrugations and metal foams plotted against plateau stress, $\sigma_{\mathrm{pl}}$. Metal foam data and tube upper bound tube predictions are presented from Ashby et al. [38]. Each foam is labeled with its density in $\mathrm{Mg} / \mathrm{m}^{3}$.

Figure 79: a) Loading diagram for the determination of effective elastic compressive modulus b) illustration of strut deflection assuming small strain and pin jointed approximation and c) free body diagram of strut loading.

Figure 80: The strut collapse model is developed to predict densification strain of the corrugated sandwich panels. The densification strain is reached when the deformed strut aspect ratio and inclination angle result in cell strengths sufficient to support the structures initial peak strength.

Figure 81: The compression set-up for the hybrid sandwich panel provides only a rear face boundary condition during testing.

Figure 82: Compressive stress-strain response for multi-tier (3-tier) glass-epoxy corrugated sandwich panel structures for the a) Divinycell H130 foam-filled structure and b) open core structure.

Figure 83: Photographs taken during compression of the multi-tier corrugated sandwich structure (Design 3, $t / l=0.14$ ). Many failure modes are observed including truss Euler buckling, microbuckling, nodal rotation, outer edge nodal shear, and matrix crazing. Parts a-f correspond to the foam-filled structure and parts g-l correspond to the open core structure. 
Figure 84: The modulus response of the corrugated structures compared to Maiti, Gibson, and Ashby's [58] bending-dominated foam model. Modulus is normalized with the bulk material modulus of the 155

Figure 85: The strength response of the corrugated structures compared to empirically determined bending-dominated and stretch-dominated foam models developed by Maiti, Gibson, and Ashby's [58].....

Figure 86: Illustration of the dynamic compressive loading of the corrugated sandwich panels. A Kolsky Bar is used for rear face pressure measurements as a cylindrical projectile impacts the sample.

Figure 87: Sketches of the Kolsky Bar setup arrangements for dynamic testing of the (a) corrugated core specimens and (b) the E-glass test coupons cut from the struts of the corrugated cores [37].

Figure 88: Stress versus time history measured in the $28.5 \mathrm{~mm}$ diameter maraging steel Kolsky Bar during a calibration test in which a $460 \mathrm{~mm}$ long steel striker (28.5 mm diameter) was fired at Kolsky Bar at $v_{0}=6.6 \mathrm{~ms}^{-1}$. The theoretical prediction based on $1 \mathrm{D}$ elastic wave theory is included in the figure along with an inset that magnifying the early time history of the stress pulse [37].

Figure 89: The measured dynamic stress versus nominal strain responses of the E-glass material coupons for a range of impact velocities. (b) Summary of the measured peak stresses E-glass specimens as a function of applied strain rate. The compressive response of the E-glass composite was measured along the weft fiber direction [37].

Figure 90: A montage of photographs showing the sequences of deformation in the (a) foamfilled and (b) open corrugated core specimens impacted at a velocity $v_{0}=150 \mathrm{~ms}^{-1}$. Impact occurs on the top face of the specimens in the photographs, illustrating the effect of dynamic impact and failure of the stress wave to propagate sufficiently fast enough through the sample.

Figure 91: The measured dynamic stress versus nominal strain responses of the foam filled and open core corrugated specimens. The stresses are measured on the rear faces of the specimen opposite from the impacted face. Little effect is observed from the foam filed cores.

Figure 92: Summary of the measured peak stresses in the filled and unfilled corrugated core specimens as function of the impact velocity (lower $\mathrm{x}$-axis) and applied nominal strain rate (upper X-axis). The measured peak stresses in Divinycell H130 foam are also included.

Figure 93: Photograph of the vertical pendulum rig used for rear-face supported testing of a core when loaded by high explosive driven high velocity sand. 172 
Figure 94: A diagram depicting the design of the explosive charge designed to accelerate watersaturated microspheres onto the front face of the specimens [128].

Figure 95: Photographs of the vertical pendulum during a test, illustrating the movement of the vertical pendulum after loading. 175

Figure 96: An illustration of the vertical pendulum set-up, showing the relative positioning of the explosive charge assembly, sample, sliding aluminum bar assembly, and soil containment box. The vertical pendulum measures transmitted impulses through the vertical height measurement of a sliding bar assembly after blast loading occurs... 176

Figure 97: A series of pressure films, designed to change color depending on the applied load, were used to measure sample pressures during vertical pendulum sliding tests. ..... 179

Figure 98: Rigidly fixing the vertical pendulum to the frame allows the aluminum bars to be instrumented with strain gauges and used as Kolsky Bars to measure rear face pressures and impulse of the structure.

Figure 99: The stress wave developed in the Kolsky Bars transverses through the material providing a clean strain signal until the stress wave reflection returns to the strain gauges, approximately $598 \mu \mathrm{sec}$ after the initial measurement.

Figure 100: The a) front edge of the high velocity glass microspheres is plotted as function of time, showing a linear response and b) constant velocities based on the explosive charge quantity (relative to the Detasheet only).

Figure 101: High speed photography captures the sand front of the glass microspheres impacting the hybrid corrugated sandwich structure while the Kolsky Bars measure rear-face stresses [134].

Figure 102: Photographs showing progressive structure damages for a series of samples tested at $14 \mathrm{~cm}$ standoff and 300 gram Detasheet explosive charge for structures with varying quasi-static plateau strengths, $\sigma_{\mathrm{pl}}$, including a) the Divinycell H130 foam-only panel ( $\left.\left.\sigma_{p l}=3 M P a\right), b\right)$ open core $\bar{\rho}=15 \%$ panel $\left.\left(\sigma_{p l}=4 M P a\right), \mathrm{c}\right)$ open core $\bar{\rho}=27 \%$ panel $\left(\sigma_{p l}=6 M P a\right)$, and d) foam-filled $\bar{\rho}=27 \%$ panel $\left(\sigma_{p l}=12 M P a\right)$. 184

Figure 103: Failure modes observed in the crushing of the hybrid corrugated sandwich panels included strut microbuckling, fractured fiber kinking, facesheet shear, matrix crazing, nodal rotation, and both shear and compressive failure. Failure is always more predominant in the top (impact side) tier with progressive failure through the structure 
Figure 104: Photograph comparison of hybrid foam-filled and open core structures tested at 14 $\mathrm{cm}$ standoff with either a 300 gram or 400 gram Detasheet charges to illustrate the failure observed in varying core strength panels. Full densification and core failure occurs at the low density cores while only partial core (top tiers) is observed for cores of higher strength. Facesheet failure was observed in all test cases. 186

Figure 105: Vertical height displacement comparisons of sliding pendulum for the hybrid glass fiber corrugated core (GFCC), $\sigma_{y}=12 \mathrm{MPa}$, at standoff distances of a) $14 \mathrm{~cm}$, b) $19 \mathrm{~cm}$, c) $24 \mathrm{~cm}$, and d) $29 \mathrm{~cm}$ standoff distances using a $300 \mathrm{~g}$ Detasheet charge. 188

Figure 106: Pressure-time response obtained from the Kolsky Bar measurement technique tested at a $14 \mathrm{~cm}$ standoff with a $300 \mathrm{~g}$ Detasheet charge for a) the solid aluminum calibration block, b) foam-filled core, c) open core, and d) Divinycell H130 foam only samples.

Figure 107: Deformed Divinycell H130 foam samples with composite (non-rigid) front facesheet after being tested at different standoff and explosive charges (i.e. different applied impulse levels). 192

Figure 108: Comparison of peak pressure measurements from the pressure film and Kolsky Bar methods show nearly a $4 \mathrm{x}$ difference for all impulse levels.

Figure 109: The pressure film provided a method to analyze both the rear-face and front-face pressure distribution. a) The rear-face pressure measurement shows localized pressures in the center of the core. The front-face pressure on the solid calibration block shows the pressure distribution using the b) medium grade (10-50 MPa), c) high grade (50-130 MPa), and d) super-high grade (130-300 MPa) pressure films. 194

Figure 110: Peak pressures measured by the Kolsky Bars on the vertical pendulum while in fixed mode shows rear-face pressure reductions until full densification is reached during dynamic crushing, at which time pressure spikes are observed.

Figure 111: Impulse measurements can be measured in the Kolsky Bar pressure-time response, but clean measurements can only be made before the stress wave reflection in the material returns to the strain gauge position. Results shown are for a $14 \mathrm{~cm}$ standoff and $300 \mathrm{~g}$ Detasheet charge.

Figure 112: Normalized impulse levels for the sandwich structure cores as measured through use of the pressure measurements from the Kolsky Bars, plotted against the normalized quasi-static core strength over the incident dynamic pressure of the calibration block as measured by the Kolsky Bars. All samples shown have an S2-glass composite facesheet. 
Figure 113: Normalized impulse levels for the sandwich structure cores as measured by the vertical pendulum (sliding mode) and plotted against the normalized quasi-static core strength over the incident dynamic pressure of the calibration block as measured by the Kolsky Bars. Several samples with rigid facesheets were tested to investigate the facesheet effect and are included in this figure and denoted as such. 199

Figure 114: The shape design can affect the transmitted impulse of dynamic soil loading on a structure.

Figure 115: Divinycell H130 foam samples with a $12.7 \mathrm{~mm}$ aluminum (rigid) front facesheet were tested at different standoff distances and explosive charges to vary the applied impulse level. These samples showed a reduced front face deformation by the removal of the concave deformation observed with the composite front facesheet. 202

Figure 116: Peak and permanent strains measured for the Divinycell H130 foam. A nickel-foam core inside of the sample was used to measure peak strains.....

Figure 117: Normalized impulse measurement for the hybrid corrugated cores with rigid aluminum front facesheets added to minimize facesheet effects as measured by the vertical pendulum (sliding mode) and plotted against the relative density. 205

Figure 118: Advanced Finite Element Analysis (AFEA) can be used to predict the high explosive blast interaction with air, soil and structural elements. Primary failure mechanisms can be estimated before testing even begins.

Figure 119: Pretension placed on the fibers could straighten the core struts and increase the fiber volume fraction, creating a much stronger corrugated cellular structure.

Figure 120: Syntactic foams used within the core could provide increased strength and energy absorption while maintaining a lightweight structure as well as provide a synergetic effect to strut buckling stabilization. 


\section{List of Tables}

Table 1: Mechanical properties of reinforcement fiber materials [22, 23]

Table 2: Design parameters of the E-glass fiber corrugated core along with the measured panel core density and relative density of the test structures with measured and predicted values for the open corrugated core.

Table 3: $3 \mathrm{Weave}^{\circledR}$ fiber distribution in the warp, weft, and $\mathrm{z}$ tows for both E-glass and S2-glass. 66

Table 4: Infusion and pressure differential transition cure cycle.

Table 5: Measured initial average fiber misalignment angles throughout the warp and weft tows in single laminate struts as measured with XCT imaging corresponding to Figures 4 and 7 for E and S2, respectively. This study reveals the peak misalignment angles do not primarily control the critical failure strength and an average is a better representation of the overall tow misalignment. 95

Table 6: Measured densification strain values of the tested structures based on the relative density of the composite core web.

Table 7: Approximate energy absorption efficiencies for the composite sandwich structures and Divinycell H130 foam.

Table 8: Replicas of the E-glass struts within the corrugated cores were manufactured and tested to provide "as-manufactured" strut properties for modeling the overall structure properties.

Table 9: Predicted densification strain, core strength, and energy absorption from the developed micromechanical models. Average dimensional parameters for each core geometry were used in the prediction. The hinge position (position of strut hinge at time of densification), $\mathrm{x}$, was experimentally measured for each core geometry and utilized as an empirical fit in the hinge model.

Table 10: Summary of the strength data for the foam-filled and open core multi-tier panels... 149

Table 11: Corresponding impulse measurements and reductions for the vertical pendulum heights for the three-tier corrugated core (Design 3: $\sigma_{\mathrm{pl}}=12 \mathrm{MPa}$ ) and corresponding calibration block along with the corresponding impulse reduction. 


\section{List of Symbols}

\begin{tabular}{|c|c|}
\hline A & cross sectional area \\
\hline$A_{\text {warp }}$ & area occupied by warp tow laminate \\
\hline$A_{\text {weft }}$ & area occupied by weft tow laminate \\
\hline$\beta$ & kink band inclination angle \\
\hline c & node thickness \\
\hline$c_{\text {long }}$ & longitudinal elastic wave speed \\
\hline $\mathrm{C}_{1}$ & constant of proportionality \\
\hline $\mathrm{C}_{2}$ & constant of proportionality \\
\hline$\delta$ & displacement/deflection/elongation \\
\hline$\delta_{1}$ & strut deflection \\
\hline$\delta_{33}$ & through thickness core deflection \\
\hline$\varepsilon$ & strain \\
\hline$\varepsilon_{D}$ & densificaton strain \\
\hline$\dot{\varepsilon}$ & strain rate \\
\hline $\mathrm{E}$ & elastic (Young's) modulus \\
\hline$E^{*}$ & elastic modulus of foam \\
\hline$E^{\prime}$ & storage modulus \\
\hline$E^{\prime \prime}$ & loss modulus \\
\hline$E_{c}$ & elastic modulus of the composite \\
\hline$E_{s}$ & elastic modulus of bulk/solid material \\
\hline$E_{f}$ & modulus of the fiber \\
\hline$E_{m}$ & modulus of the matrix \\
\hline$E_{\text {weft }}$ & modulus of the weft fiber tow \\
\hline$E_{\text {warp }}$ & modulus of the warp fiber tow \\
\hline$E_{\text {strut }}$ & modulus of the strut \\
\hline$E_{\text {core }}$ & through thickness modulus of open core \\
\hline$E_{f f-c o r e}$ & through thickness modulus of foam-filled core \\
\hline$f_{\text {warp }}$ & fractional area of warp fiber tows \\
\hline$f_{\text {weft }}$ & fractional area of weft fiber tow \\
\hline$F_{33}$ & through thickness core loading \\
\hline$F_{t}$ & axial strut loading \\
\hline$F_{v}$ & transverse strut loading \\
\hline$\phi$ & initial average fiber tow misalignment angle \\
\hline $\mathrm{g}$ & gravitational acceleration \\
\hline
\end{tabular}




\begin{tabular}{|c|c|}
\hline$G^{*}$ & shear modulus of foam \\
\hline$h_{f}$ & facesheet thickness \\
\hline$h_{f}$ & final height of vertical pendulum rig \\
\hline $\mathrm{H}$ & core height \\
\hline I & impulse \\
\hline $\mathrm{j}$ & momentum \\
\hline $\mathrm{k}$ & preform permeability \\
\hline $\mathrm{K}$ & effective length factor/clamping condition coefficient \\
\hline$K E_{o}$ & initial kinetic energy \\
\hline$K E_{f}$ & final kinetic energy \\
\hline 1 & strut length \\
\hline $\mathrm{L}$ & strut length \\
\hline $\mathrm{L}_{\mathrm{p}}$ & half width of panel blast panel \\
\hline $\mathrm{m}$ & mass \\
\hline$n_{1}$ & exponential proportion constant \\
\hline$n_{2}$ & exponential proportion constant \\
\hline $\mathrm{P}$ & pressure \\
\hline$P$ & applied loading \\
\hline$P_{\text {weft }}$ & load on weft tow laminate \\
\hline$P_{\text {warp }}$ & load on warp tow laminate \\
\hline$P E_{o}$ & initial potential energy \\
\hline$P E_{f}$ & final potential energy \\
\hline$\rho$ & density \\
\hline$\rho_{s}$ & density of solid/bulk material \\
\hline $\bar{\rho}$ & relative density \\
\hline$\rho^{*}$ & density of foam \\
\hline $\begin{array}{l}\mathrm{r} \\
\sigma\end{array}$ & $\begin{array}{l}\text { standoff distance between the explosive center and front face } \\
\text { stress }\end{array}$ \\
\hline$\sigma_{c}$ & critical strength \\
\hline$\sigma_{c r}^{*}$ & crushing/fracture strength \\
\hline$\sigma_{c r, s}{ }^{*}$ & tensile fracture strength of bulk solid \\
\hline$\sigma_{e l}^{*}$ & elastic buckling collapse strength \\
\hline$\sigma_{\text {Euler }}$ & Euler buckling strength \\
\hline$\sigma_{f}$ & yield strength of composite loaded axially along fibers direction \\
\hline$\sigma_{f s}$ & modulus of rupture of the cell wall/strut \\
\hline$\sigma_{p l}$ & plateau strength \\
\hline$\sigma_{y}$ & yield strength \\
\hline$\sigma_{y s}$ & yield strength of bulk/solid material \\
\hline
\end{tabular}




$\begin{array}{ll}\sigma_{y}^{*} & \text { yield strength of the cellular material } \\ \sigma_{\text {core }} & \text { compressive strength of the core } \\ \sigma_{\text {critical }} & \text { critical strength of the strut } \\ \sigma_{f f-c o r e} & \text { strength of the foam-filled core } \\ \sigma_{\text {warp }} & \text { warp fiber tow laminate strength } \\ \sigma_{\text {weft }} & \text { weft fiber tow laminate strength } \\ \sigma_{33-c r i t} & \text { critical through thickness strength } \\ \psi & \text { porosity } \\ \mathrm{t} & \text { time } \\ \mathrm{t} & \text { strut thickness } \\ \mathrm{T}_{\mathrm{g}} & \text { glass transformation temperature } \\ \mathrm{T}_{\mathrm{m}} & \text { melting temperature } \\ \tau & \text { shear strength } \\ \tau_{m} & \text { matrix shear strength } \\ \mathrm{tan} \delta & \text { ratio of loss modulus to the storage modulus } \\ v_{f} & \text { fiber volume fraction } \\ v_{f a} & \text { fiber volume fraction of axial fibers } \\ v_{m} & \text { matrix volume fraction } \\ v_{o} & \text { initial velocity } \\ v_{f} & \text { final velocity } \\ \mathrm{V} & \text { interstitial velocity } \\ V_{u n i t C e l l} & \text { volume occupied by the unit cell } \\ V_{\text {core }} & \text { volume occupied by the core material } \\ \mathrm{U} & \text { absorbed energy } \\ \mu & \text { fluid viscosity } \\ \omega & \text { orientation angle of strut } \\ \mathrm{X} & \text { resin front distance } \\ & \end{array}$




\section{Chapter 1}

\section{Introduction}

\subsection{Rationale for the Study}

Structures capable of mitigating high pressures and impulses generated through dynamic loading are needed to protect both personnel and equipment in a high explosive blast threat environment. Threats from Improvised Explosive Devices (IEDs) are the greatest impetus for investigating alternative armor technologies. IEDs accounted for over 50\% of hostile fatalities during Operation Enduring Freedom between 2001 and 2014 [1] . As a result of the persistent threat from these weapons, interest in novel, high performance structures capable of energy absorption, impulse mitigation, through-structure pressure reductions, and blast deflection as a potential use as vehicle armor (or for a use in conjunction with an existing armor) has grown significantly throughout the past two decades for both land and sea vehicles. Specific interest exists to develop underbody armor for vehicles designed to protect the vehicles structure which, in turn, will help protect the soldier.

Strength, survivability, weight, and cost are essential factors to developing a viable armor technology. These parameters provide guidelines for basic design and development of an impulse mitigating structure and lead a designer to consider modern composite materials as a 
solution. Modern advanced composite materials provide a wide range of material properties capable of peak strengths 15 times greater and moduli ${ }^{1} 10$ times greater than steel [2] counterparts, but historically costing much more than an equivalent aluminum or steel counterpart. While composite materials have been under development for decades, technological advances in materials and manufacturing methods along with reduced production costs have opened more opportunities for composite materials in recent years. The use of composite materials has grown and now is frequently used in the transportation, construction, and marine industries, as well as applications related to electrical and corrosive protection [2]. The adaptability of composites to unique structural applications requiring strict mechanical performance has driven the desire for composite use in military applications including applications ranging from vehicle [3] and body armor [4] to structural members in air and land vehicles [5].

The increased capability and lethality of terrorist groups against military assets has grown over the past 15 years. On October 12, 2000, the USS Cole was attacked by a small boat with explosives while refueling in Aden Harbor in Yemen. The attack resulted in a 40ft x $60 \mathrm{ft}$ hole blown into the side of the USS Cole, Figure 1(a), killing 17 sailors and immediately crippled the vessel. Even more devastating has been the IED threat against land vehicles in the Middle East regions. Throughout Operation Enduring Freedom alone, there have been nearly 1400 fatalities due to IEDs. Rigorous design in blast protection vehicles has been underway for decades to provide safe transport of personnel and equipment. Even some of the best designs deployed today are still vulnerable to IED attacks, such as the Cougar Mine-Resistant Ambush Protected

\footnotetext{
${ }^{1}$ Properties for strength and modulus are for quasi-static loading.
} 
(MRAP) vehicle, as shown Figure 1(b). As a result, continued interest in blast armor technologies continues to this day.
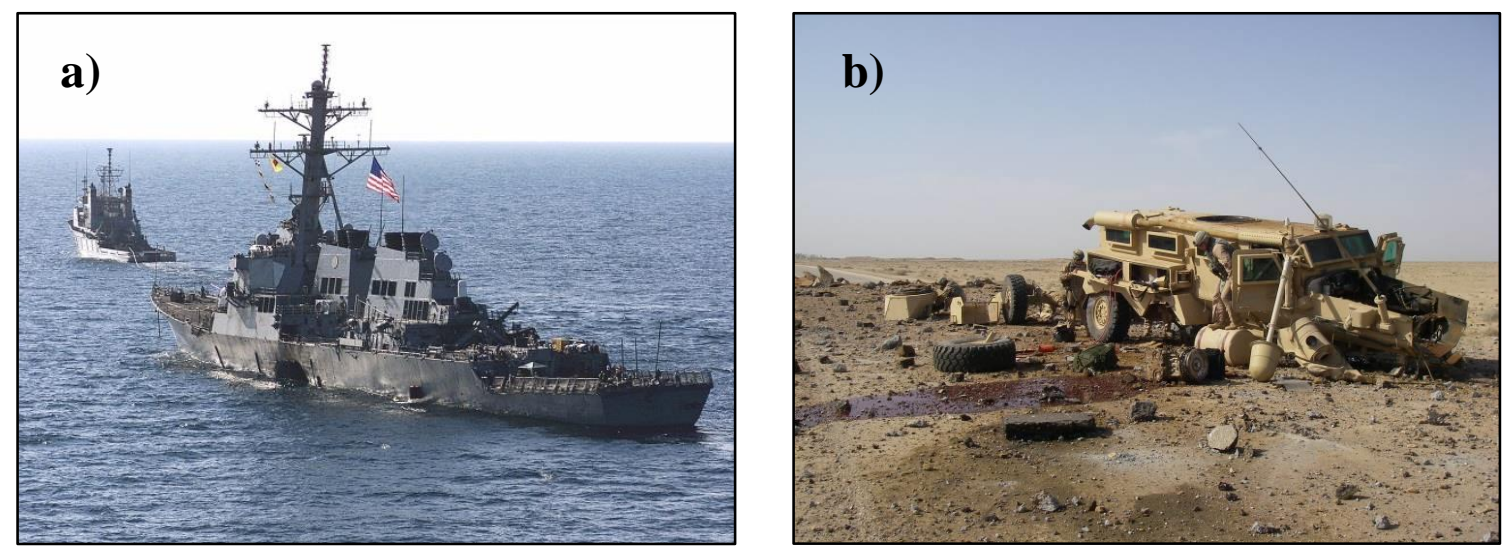

Figure 1: Explosive devices have been used by terrorist to damage both sea and land vehicles such as a) the USS Cole [6] and b) the Cougar MRAP vehicle [7].

In the development of an armor technology, a thorough understanding of the loading scenario is required. Due to the high loads that are generated in dynamic blast events, a panel designed for a specific loading scenario might perform very poorly if that scenario is altered. In the case of an IED detonating under a vehicle, not only is the protection against blast waves critical, but protection from fragmentation is also needed. Buried IEDs rely on soil and debris to be propelled at high velocities toward the vehicle. The energy transfer of the blast shock wave and generated explosive gases to this debris and sand/soil ejecta will greatly affect the lethality of the IED. Many factors contribute to the effectiveness of the energy transfer including explosive type, explosive quantity, depth of burial, moisture content of the soil, and the boundary conditions of the soil surrounding the IED. A tangential effort to the research of the composite sandwich structure has investigated the parameters associated with buried IED effectiveness. 
The research presented in this study focuses on a single explosive scenario for the scientific analysis of the structure itself.

Academic research on blast events typically focuses on idealized, scaled blast to investigate the mechanical response of structures that may exhibit viability as an armor technology. While this tact creates results non-specific to an actual threat, the method allows for research to progress at a non-classified level to provide a basis for understanding the mechanics and failure mechanisms of a structure. Academic research studies on armor technologies are typically simplified by avoiding a specific vehicle-armor design and focuses on research of the armored panel only. As a result, representative boundary conditions are created to evaluate the structures response in a realistic manner.

The boundary conditions of the panel prove to be equally as important to the overall performance of the structure as the IED parameters and mechanical structure \& design of the armor panel. Common boundary conditions include an edge-clamped scenario, Figure 2(a), and a rear-face supported scenario, Figure 2(b). The edge-clamped boundary condition allows the structure to bend and stretch under testing, inducing a complex internal loading response. Rearface supported boundary conditions investigate the crushing behavior of a panel with the stretching of the overall panel minimized. Edge-clamped scenarios provide a more representative case of the survivability of a structure under dynamic conditions, providing a measurement of structure deflection and failure mechanism [8, 9]. Rear-face supported conditions allow for dynamic pressure and impulse measurements to be determined [10]. In the implementation of an armor technology, specific boundary conditions would need to be evaluated. 

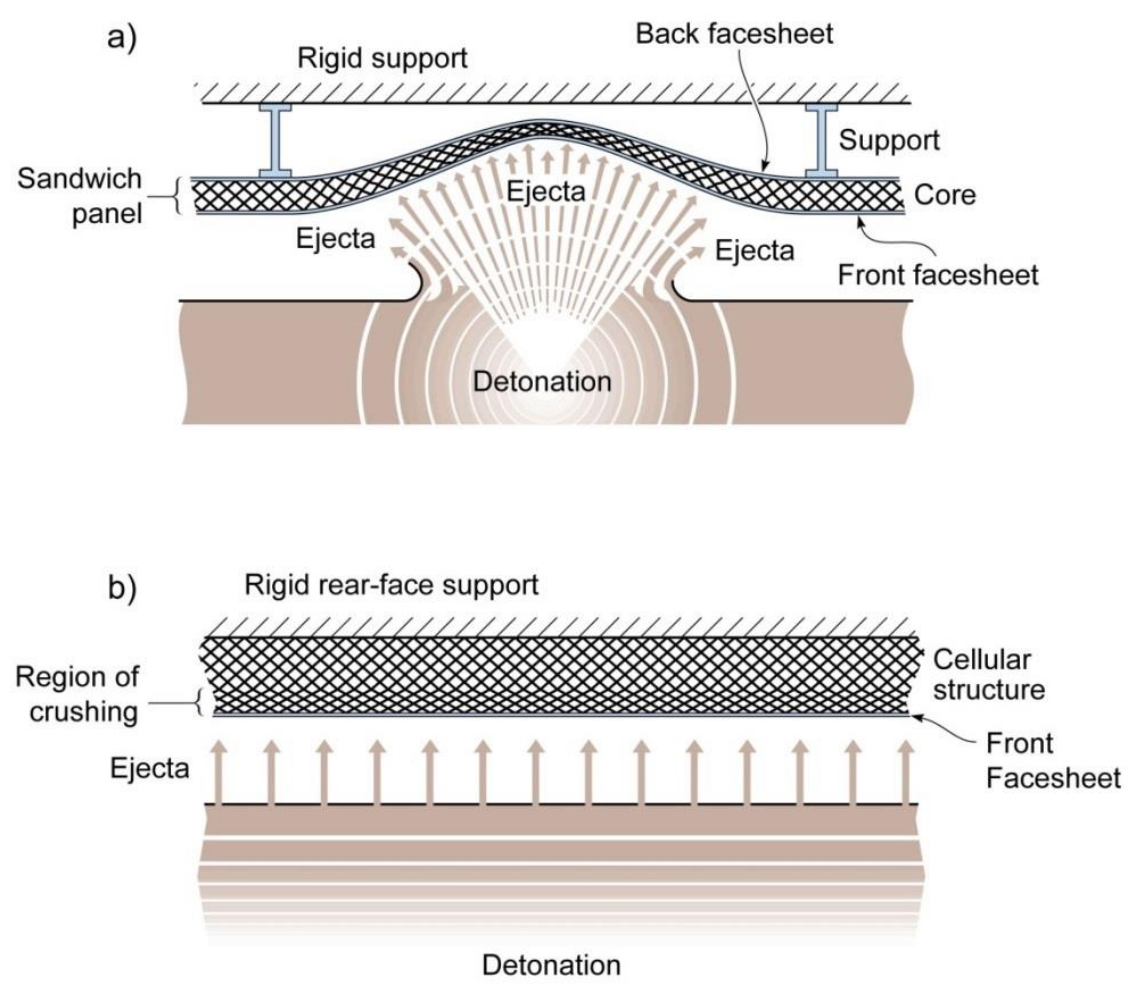

Figure 2: Common boundary conditions associated with explosive blast testing include a) edge-clamped conditions allowing for panel stretching and deformation and b) rear-face supported conditions used to evaluate panel crushing.

Armor technologies have been created from a multitude of materials, with the most common armors being created from sheets of bulk metal steel or aluminum. These metal sheet armors, while survivable in a blast event, lack the desired ability to absorb energy and provide through structure pressure reduction. As a result, shock waves can pass through the material imparting significant damage on the internal structure and personnel. Building on the premise that an armor technology needs to absorb energy, versus transmit the energy, along with a combined motivation to reduce weight, ultralight materials and structures are being investigated as a solution. Foams materials show excellent energy absorption ability in compressive applications. Existing research has combined foam materials with rigid, strong face sheets to 
create a sandwich panel system for use in impulsive loading applications $[11,12]$. Alternately, researchers have maintained the use of common metal, including steel and aluminum, as the material of construction, but designed and created a periodic cellular structure sandwich panel to minimize weight and create localized internal areas where failure initiates and is able to absorb and dissipate the imparted blast energy [13-15]. Both structures show potential as a viable blast armor technology and are the basis for this research.

\subsection{Hybrid Corrugated Sandwich Panel - Preliminary Blast Loading}

The preliminary design concept for the hybrid composite corrugated sandwich panel was built from the energy absorbing foam sandwich panel designed by Latourte [11] and Zenkert et al. $[11,12,16]$. Their structure uses a foam core to provide energy absorption, compressive strength, and facesheet separation for loading in both edge-clamped and rear-face supported scenarios. Specifically, Latourte and Zenkert use a closed-cell PVC Divinycell H250 series foam with laminated E-glass composite facesheets to provide structural resistance to impulsive loading underwater [11]. It was concluded that a performance improvement was observed at sufficiently high impulse per areal mass ratios of the sandwich panel versus the solid composite panels. Primary failure mechanisms included extensive delamination of the composite facesheets and crack propagation through the foam-composite interface, foam core, and composite facesheets.

The sandwich structure developed by Latourte and Zenkert et al. was a simple concept not intended for an armor technology but rather for the pursuit of scientific knowledge. Regardless, it showed potential for survivability under dynamic loading, energy absorption, and 
impulse mitigation. Simultaneously, research on complex cellular structure sandwich panel designs were being studied to better understand the advantages of changing the internal structure response from a bending-dominated to stretch-dominated failure mechanism [17]. Recent theoretical [18] and experimental $[19,20]$ studies of impulsively loaded, edge clamped metallic panels have shown that sandwich panel concepts with cellular cores suffer even smaller deflections than equivalent (same mass per unit area) solid plates, provided the cellular core is sufficiently strong to maintain face sheet separation (and sandwich panel action) during loading.

The hybrid composite corrugated sandwich panel concept modifies the basic foam structure developed by Latourte and Zenkert et al. and selectively replaces select areas of foam with a periodic corrugated cellular structure manufactured from composite materials. The design intent was to exploit the higher compressive strength provided by a corrugated cellular structure made from advanced composite materials. This change is hypothesized to alter the failure mechanism from a bending dominated mechanism (as seen by stochastic foams) to a stretchdominated failure mode (as seen in cellular structures). The results are hypothesized to increase the modulus, increase the critical compressive strength, and provide strut stabilization from the surrounding foam which could provide a synergetic strengthening effect. The foam core is also hypothesized to provide increased energy absorption and is a key component to facilitate the successful manufacture of a dry fabric structure. The resulting preliminary design uses E-glass to manufacture a corrugated composite core integrated with PVC foam and bonded through $\operatorname{Kevlar}^{\circledR}$ stitching and matrix infusion to stronger S2-glass facesheets, Figure 3. Details on the hybrid foam glass-epoxy composite corrugated sandwich structure are presented later throughout this research work. 


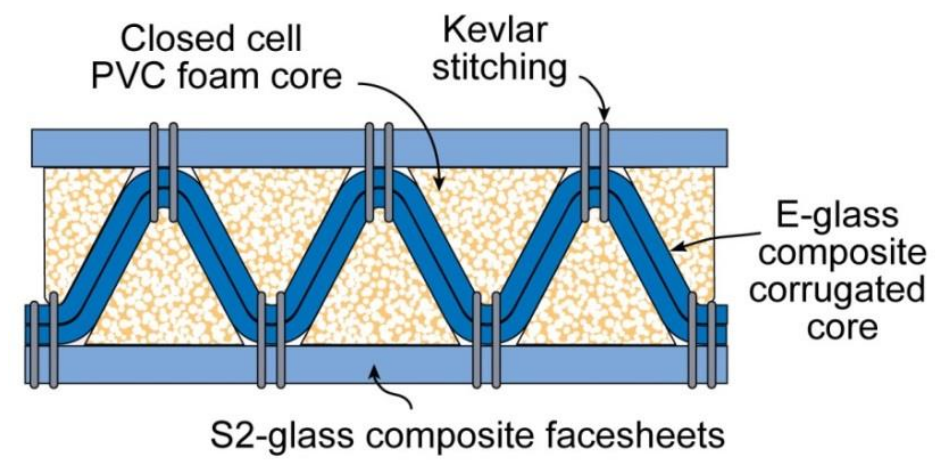

Figure 3: The substation of a portion of a foam core sandwich structure with a periodic corrugated cellular structure resulted in the hybrid foam glass-epoxy composite corrugated sandwich panel concept developed for analysis as an armor technology.

The opportunity to test large scale panels under an edge-clamped boundary condition was available early in the research to provide experimental analysis in a high explosive sand blast environment. After initial design and development of the hybrid sandwich panel, this structure was immediately manufactured to provide insight on the failure mechanisms, flaws, and limitations of the design. The sample panels were fabricated to be $406 \mathrm{~mm}$ (16 in) by $406 \mathrm{~mm}$ (16 in) with an additional 101.6mm (4 in) aluminum metal frame integrated around the panel resulting in a $610 \mathrm{~mm}$ square panel ( $2 \mathrm{ft}$ square), Figure 4 . The test section was made from a Divinycell H130 PVC foam core with an integrated E-glass corrugated core constructed from a dual laminate of three-dimensional (3D) woven fiber mat (areal density $3.66 \mathrm{~kg} / \mathrm{m}^{2}$ ) and bonded to an S2-glass 3D woven fiber mat facesheets (areal density $3.29 \mathrm{~kg} / \mathrm{m}^{2}$ ) through both Kevlar ${ }^{\circledR}$ stitching and matrix infusion. A rubber toughened epoxy matrix was used (see section 3.2.4 for further details). 


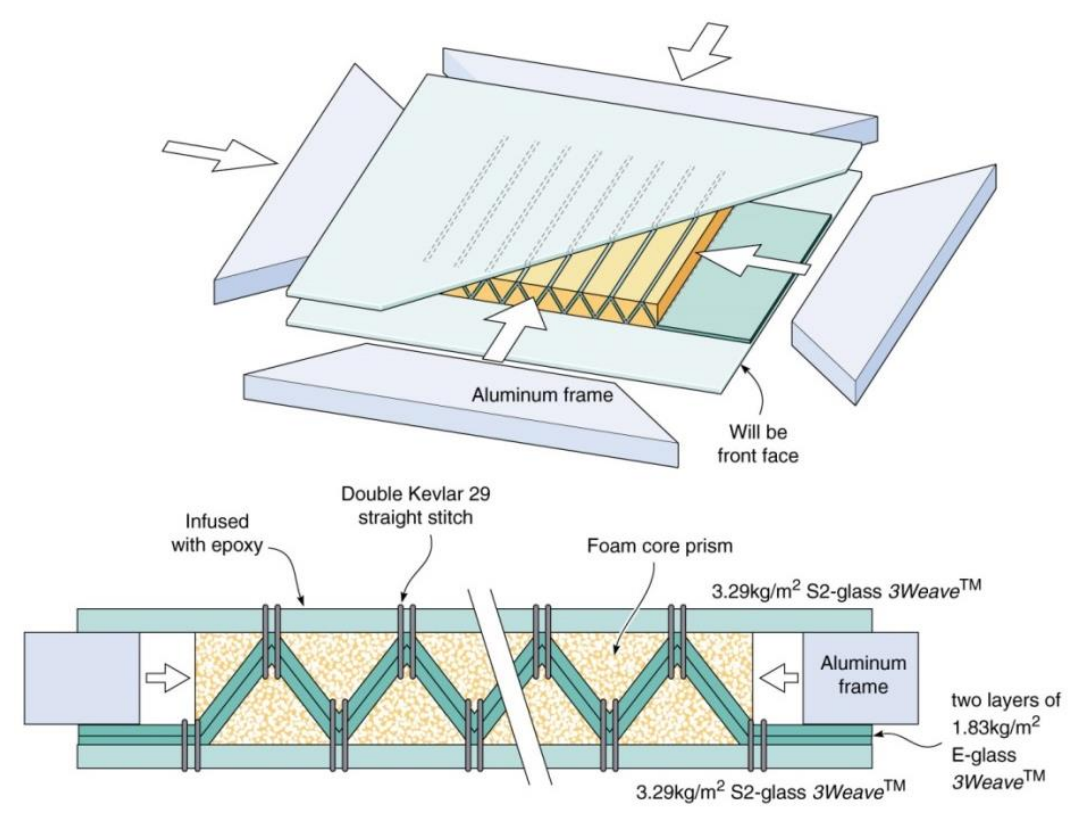

Figure 4: Edge clamped corrugated core blast panel

The resulting panels were $25.4 \mathrm{~mm}$ in thickness with a resulting areal density of the composite core of $27 \mathrm{~kg} / \mathrm{m}^{2}$. The S2-glass facesheets where manufactured to extend over the aluminum mounting frame and through holes were manufactured to the necessary specifications of the test fixture. A sample edge-clamped test specimen is shown in Figure 5(a).
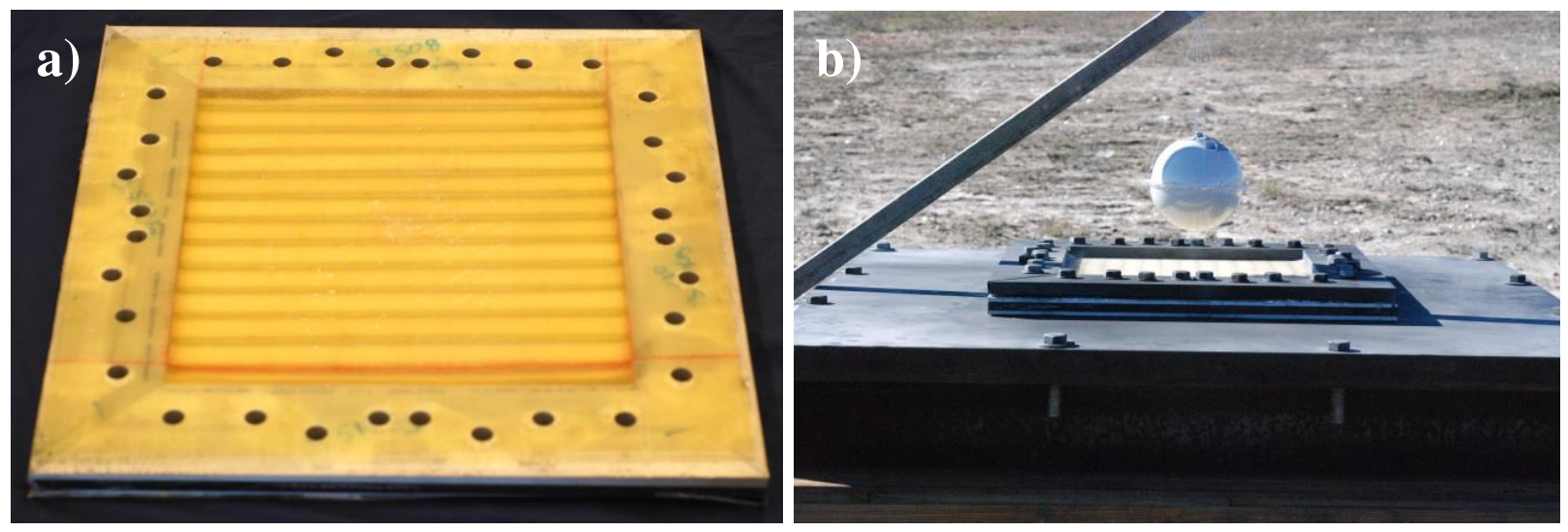

Figure 5: Hybrid foam glass-epoxy composite corrugated sandwich panel manufactured for an edge-clamp blast loading scenario. a) A top-down view of the untested panel shows the core and integrated mounting frame. b) The panel as mounted for the edge-clamped explosive sand-blast test. 
The test conditions implemented where designed to simulate a scaled version of a buried IED. The explosive charge used was 150 grams of $\mathrm{C} 4$ plastic explosive initiated by a single detonator. To simulate a buried IED, the C4 charge, was formed into a $60 \mathrm{~mm}$ sphere and centered within a $150 \mathrm{~mm}$ plastic shell. This shell was filled with $200 \mu \mathrm{m}$ diameter glass microspheres and filled to saturation with water to provide the maximum energy transfer between the explosive and the ejecta [19], see Figure 6(a). The panel is bolted to a rigid steel fixture with an aperture opening of $406.4 \mathrm{~mm} \times 406.4 \mathrm{~mm}$, Figure $6(\mathrm{~b})$ and the explosive charge/sand ball was suspended a distance of $15 \mathrm{~cm}$ from the center of the charge to the panel surface to build from prior research [20] by Dharmasena et al. A series of samples was tested against a dry and fully water saturated charges as well as a bare $\mathrm{C} 4$ explosive charge. Equivalent mass aluminum panels and laminated 3D woven panels were simultaneously tested for comparison purposes.

a) Explosive Charge Set-up

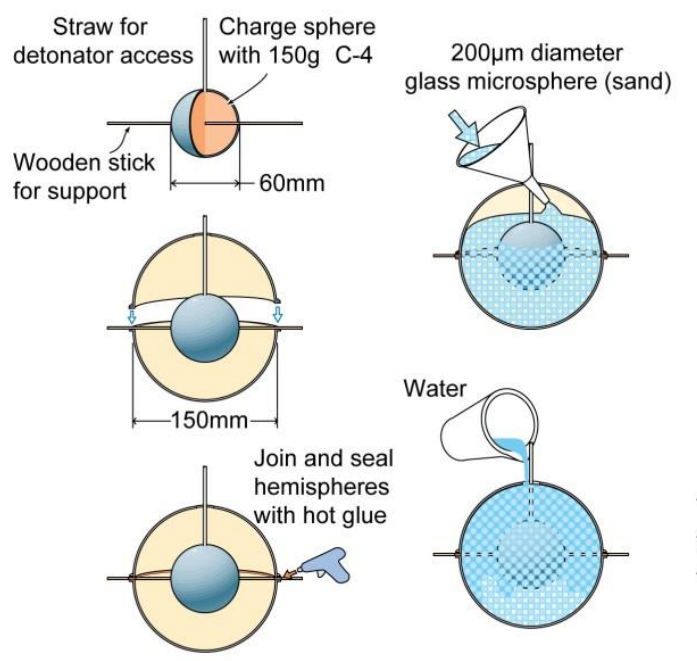

b) Experimental Test Set-up

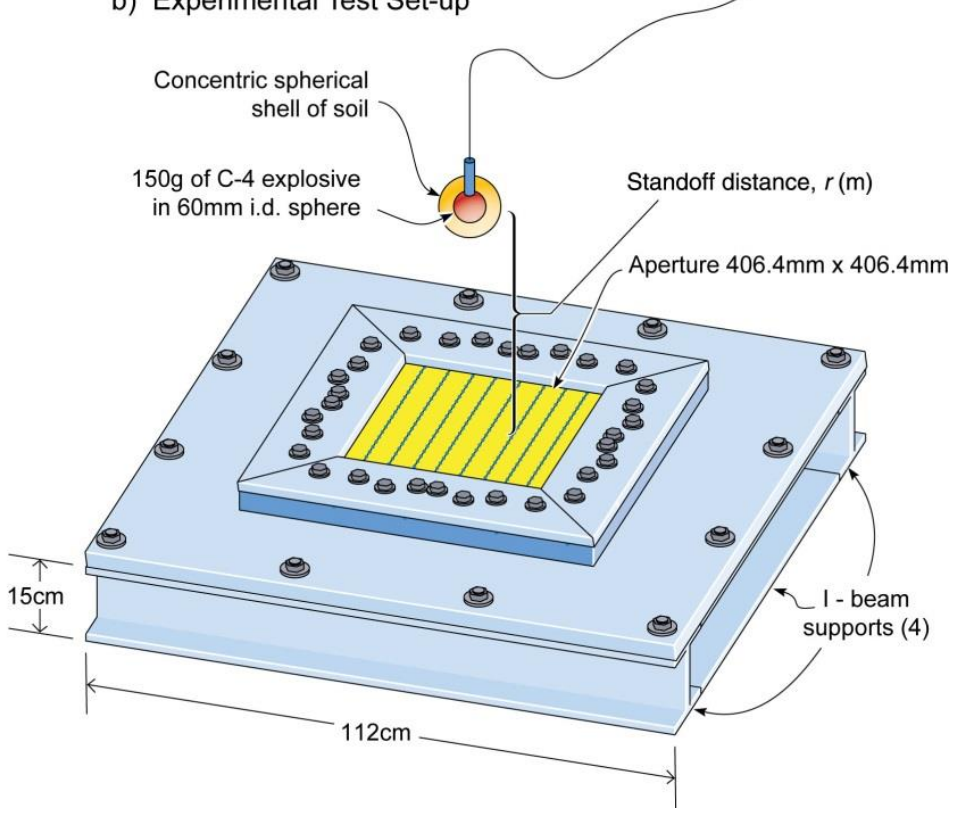

Figure 6: Edge-clamped loading scenario of the hybrid foam glass-epoxy corrugated composite sandwich panel. a) The explosive charge is created with a spherical 150 gram charge of $\mathrm{C} 4$ explosive surrounded by a $150 \mathrm{~mm}$ diameter sphere comprised of glass microspheres saturated in water. b) The sample is rigidly mounted with the explosive charge/sand ball suspended above the panel with a distance of $15 \mathrm{~cm}$ from the charge center to the panel front face. 
The results of the edge-clamped blast tests showed a positive response for the panel design. Three separate transmitted impulse levels were tested to include $0.65,3.1$, and $4.7 \mathrm{kN}$ $\mathrm{sec} / \mathrm{m}^{2}$ corresponding to $\mathrm{C} 4$ charges surrounded by no ejecta, dry ejecta, and fully saturated ejecta, respectively. The resulting rear-face deflections, $\delta$, normalized by the half width of the panel, $\mathrm{L}_{\mathrm{p}}$, are shown in Figure 7. It was discovered that the hybrid composite sandwich panel and laminated composite equivalent mass panels exhibited significantly lower normalized deflections $\left(\delta / L_{p}\right)$ relative to the equivalent mass aluminum panel at the lower impulse levels.

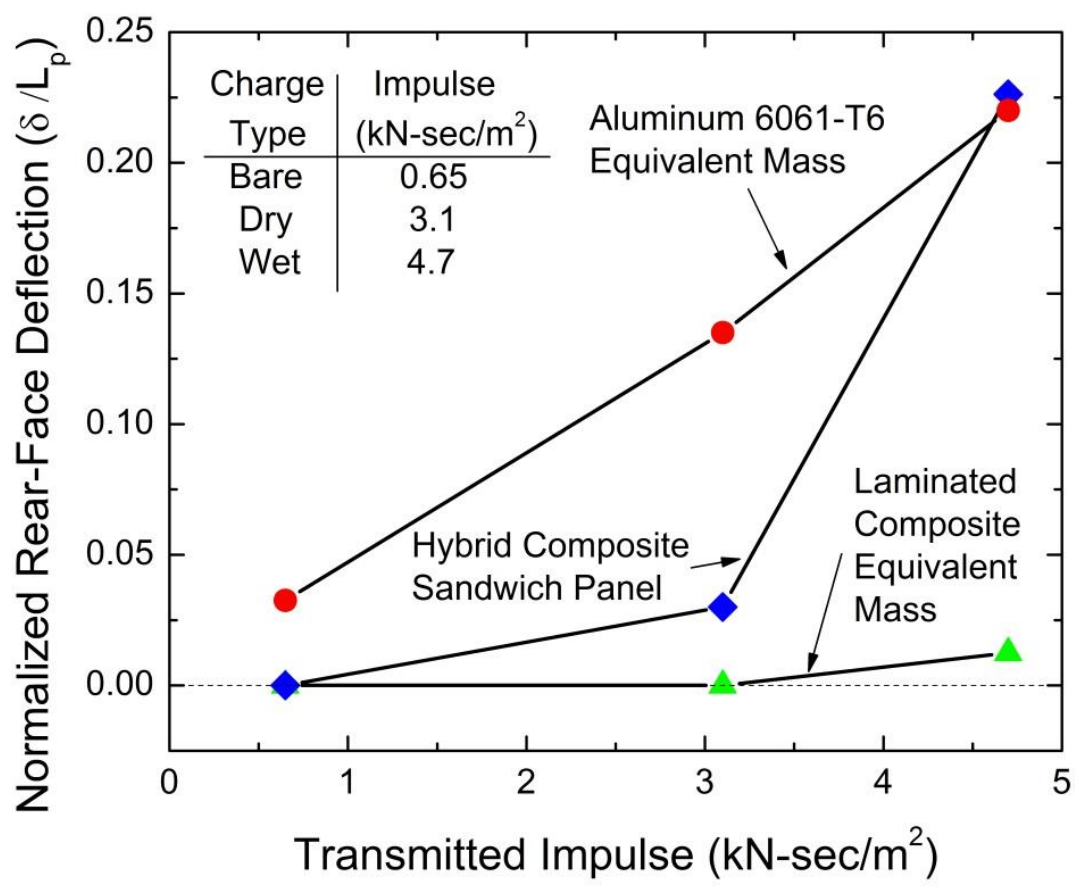

Figure 7: Normalized rear-face deflections $\left(\delta / L_{p}\right)$ measured for the hybrid composite sandwich panel and equivalent mass laminated composite and aluminum panels under three different impulse loads corresponding to a bare, dry, and wet charge type all using 150 grams of $\mathrm{C} 4$ explosive.

The rear-face deflection of the hybrid composite sandwich panel at the high impulse level was artificially high due to failure of the front and rear facesheets at the core/frame interface.

This failure, Figure 8, allowed the core to flex along the nodal points and pivot along the apexes of the corrugations. A qualitative analysis showed little change in deflections along the length of 
the prismatic core. The laminated composite equivalent mass panel showed excellent resiliency with almost zero permanent deflection measured post-blast and minimal damage observed. No delamination or crack formations where discovered beyond the surface level and only surface burning of the panel was observed when exposed to the bare charge detonation. Additional failures observed in the hybrid panel at the high impulse level include localized tensile failure at the sample surface, matrix crazing, stitch failure, and foam core/facesheet bond failure.
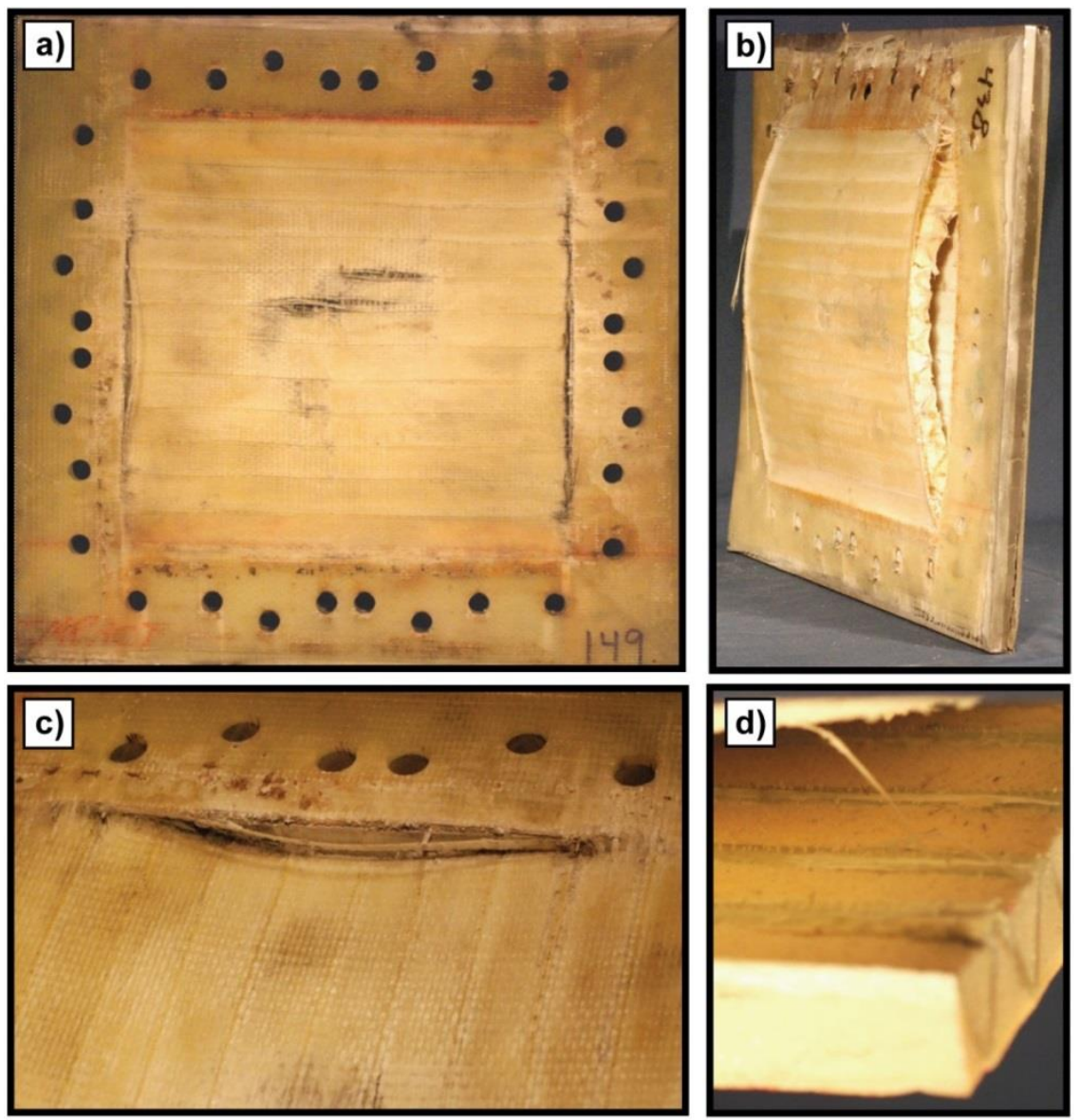

Figure 8: Post-blast images of the hybrid panel tested in an edge-clamped scenario with a 150 gram $\mathrm{C} 4$ explosive charge encapsulated in a water saturated glass microsphere soil layer. Observed failure included: a) and c) S2-glass facesheet tensile failure and burning in sample center and front facesheet shear along core/frame boundary, b) Rear facesheet shear and matrix crazing at positions of core displacement, and d) nodal failure exhibiting stitch and matrix failure along with foam core/facesheet bond failure. 
While the hybrid corrugated sandwich panel appeared to exhibit the greatest rear-face deformations at large impulse levels, the large deformations were not primarily due to core failure but rather interface failure. The edge-clamped boundary condition introduces a "point of contact" variable inherent to this test scenario. As seen in Figure 9, the aluminum mounting frame and the permanent rig that provides the necessary support for bolting the test panel rigidly to the rig provides a point of contact at the core/frame interface. When loaded, both the front and rear facesheets are loaded vertically downward, while the frame provides vertical resistance upward creating a large shear force at the interface causing failure in the fiber and matrix. This "point of contact" failure varies significantly based on the radius of the edge of the frame and rig and are less easily measured as the material of the core is altered. As anticipated, the edgeclamped scenario placed the composite facesheets under tension when loaded. It was observed that local areas of fiber tensile failure were observed in the facesheets along the perpendicular axis to the corrugated prismatic axes. Tensile failure was accompanied by matrix crazing commonly seen in composites. Finally, failure of the Kevlar ${ }^{\circledR}$ stitches at the nodes occurred closest to the outer edges of the core along with an observed shear failure of the foam core along the facesheet interface, Figure 10. The large deformations of the hybrid composite sandwich panel created a situation where the stitching was placed in a shear load as certain members of the core and facesheets where placed under tensile and compressive loading. 

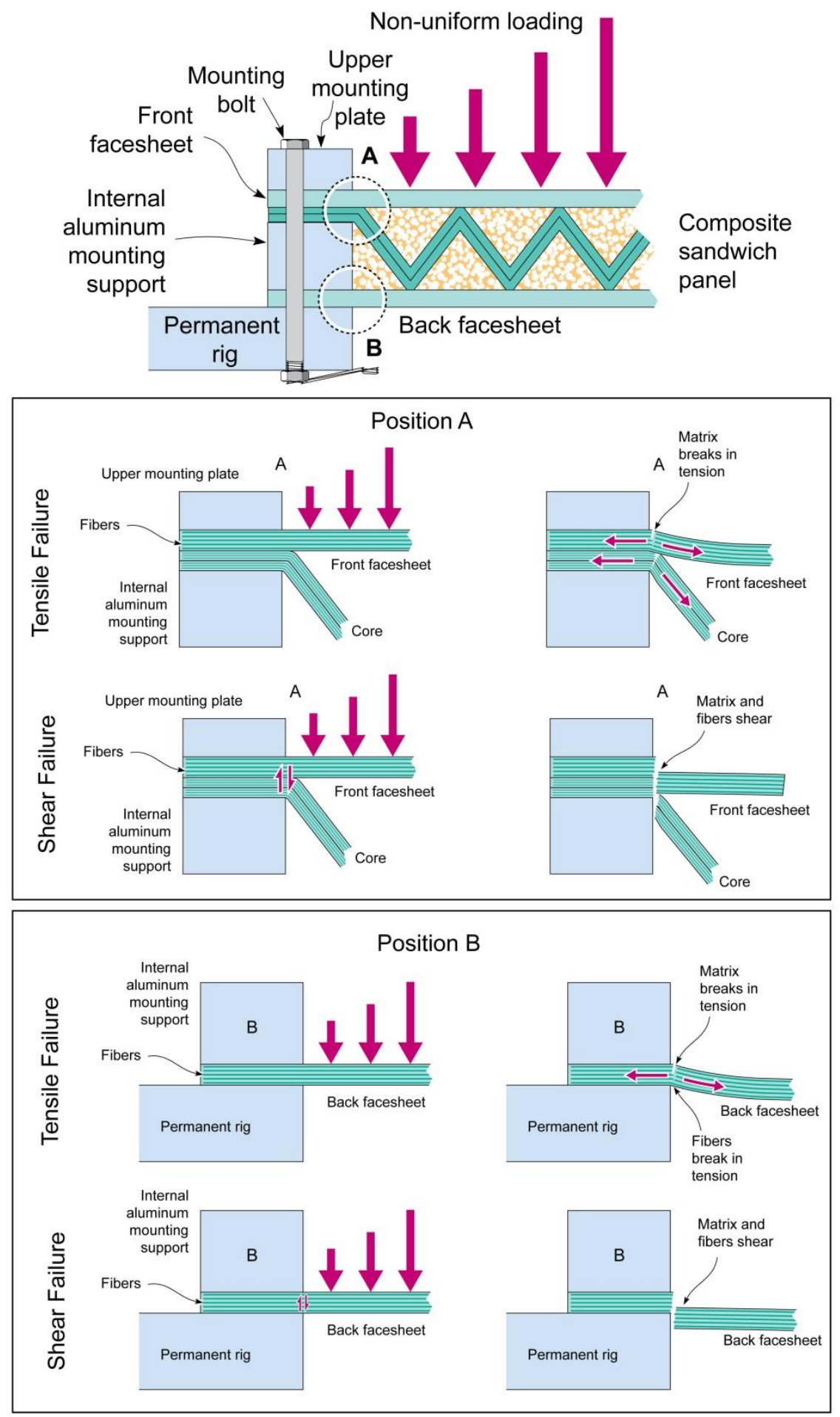

Figure 9: Analysis of the failure mechanisms of the edge-clamped test samples show that that the boundary conditions created a "point-of-contact" variable between the facesheets and either the aluminum mounting frame or permanent rig. Shear failure was prematurely induced by sharp edges that could transmit large shear loads to the facesheets. 


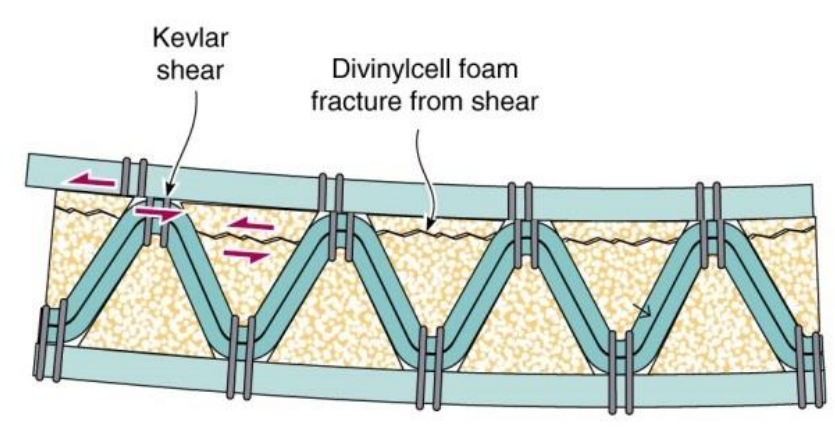

Figure 10: Shear failure was observed in the nodal stitching and Divinycell foam core resulting from the large deformations of the hybrid composite sandwich structure.

The results of the preliminary blast experiments of the hybrid composite sandwich panel show that both the composite material and the structure have significant potential for use as a blast armor. The best route to evaluating this structure in detail would be to limit the variables and create a repeatable method of loading. As a result, future dynamic tests will occur using a rear-face supported boundary condition to properly evaluate the compression response of the core itself. A detailed quasi-static study of the core is needed to understand the structural loading and mechanisms by which imperfections and failure occur. The ability to tailor advanced composite materials to alter the structural properties and behavior provides an excellent platform to designing a blast armor.

\subsection{Dissertation Goals and Outline}

This research investigates the mechanical response of a hybrid foam glass-epoxy composite corrugated sandwich panel under quasi-static and dynamic loading with rear-face supported boundary conditions. Rear-face supported boundary conditions provide the ability to instigate the through-thickness core compression, a critical starting point for underbelly vehicle 
armor. With the ultimate goal of creating a path forward for developing a viable vehicle armor for structural protection, the research goals in this study are to accomplish the following:

- Identify and characterize the quasi-static response of this novel hybrid composite corrugated sandwich panel core along with the core struts and associated constitutive materials through empirical testing.

- Develop quasi-static analytic predictions for the strength and modulus of the core and core struts.

- Empirically investigate the dynamic strength and impulse mitigation performance along with the associated dynamic failure mechanisms.

The successful development of empirically validated quasi-static loading micromechanical models will allow for the optimization of this structure in future studies. The successful measurement of dynamic strength and impulse reductions will provide the preliminary evaluation of this structure as armor for vehicle protection. Successful vehicle armor must provide through-structure pressure reduction and impulse mitigation.

This research is very significant for several reasons. First, a hybrid foam glass-epoxy composite corrugated cellular structure sandwich panel has never been manufactured or evaluated to the extent of this study. The successful manufacture of this structure will provide the ability to investigate the mechanical response of the constitutive materials, composite materials and structures, providing both empirical results and analytic predictions for the mechanical properties. Second, this research develops a single-step, all-inclusive infusion method for an entire dry fabric composite sandwich panel that can minimize both air and resin vapor voids. Third, the optical characterization of a 3D woven fabric has been poorly performed 
in other studies. This research investigates the fiber structure and fiber waviness, discovering that the average initial fiber tow misalignment is critical to the strength of the core strut. Fourth, the structures are experimentally investigated under loading both quasi-statically and dynamically to investigate the overall structure response and assess the adaptability for use as an armor technology. Finally, development of analytic predictions for strength and modulus are created for the quasi-static compression of the 3D woven composite strut and core. A new model to predict the compressive core strut strength and the hybrid core densification strain are developed and validated experimentally.

This dissertation is organized as follows: Chapter $\mathbf{2}$ presents the necessary background on advanced composites including fibers, matrix materials, and composite systems. Chapter $\mathbf{3}$ provides background on energy absorbing materials and structures including foam materials and periodic cellular structures along with the adaptation of these materials/structures into sandwich panel structures. Chapter $\mathbf{4}$ describes the design of the hybrid foam glass-epoxy composite corrugated core cellular structure sandwich panel and the constitutive materials used in the fabrication of the design. Rationale and design detail on the 3D woven fabric is also presented. Chapter 5 presents the fabrication methodology of both the dry (non-infused) fabric structure and single step vacuum infusion process used to infuse the entire composite structure. Details on manufacture of base composite materials, single tier (used for quasi-static and dynamic testing) and multi-tier (used for dynamic blast testing), along with associated imperfections are presented. Chapter 6 presents details pertaining to the empirical testing and analytic modeling of the 3D woven composite strut in both tension and compression along with the material characterization of the structure using optical microscopy and X-Ray Computed Tomography (XCT). Chapter 7 is a comprehensive study of the hybrid corrugated structure under rear-face 
supported quasi-static compressive loading including empirical results and analytic predictions of the overall structure response. Chapter $\mathbf{8}$ empirically investigates the dynamic behavior of the structure under purely a rear-face supported compressive loading via a projectile and then high-explosive driven sand blast loading. Chapter 9 discusses the results and conclusions of the quasi-static and dynamic loading of the structure. Highlights on the important findings and novel contributions are presented. Finally, Chapter $\mathbf{1 0}$ presents potential improvements, future uses of the structure, suggested research paths forward, and an assessment of the structures viability for future research. 


\section{Chapter 2}

\section{High Performance Composite Materials}

\subsection{High Performance Materials}

High performance materials are characterized through their properties as related to common materials used in the industry. Many advanced composites are considered high performance in the area of mechanical response because the materials are extremely strong, stiff, and light-weight as compared to metal counterparts such as steel, titanium, and aluminum. With the capability of being designed and manufactured for high structural demand applications in the aerospace, transportation, marine, and military industries, advanced composites offer a promising solution to optimize and improve structural systems. Figure 11 and Figure 12 show the material property space as developed by Ashby [21] and provide a necessary comparison of strength, stiffness, and density for a multitude of materials including composites and metal materials. Strength versus density, plotted in Figure 11, and modulus (Young's Modulus) versus stiffness, Figure 12, reveals that some solid materials such as engineering ceramics, metallic alloys, metal matrix composites, and ceramic matrix composites are strong and stiff but exhibit relatively high densities. Materials such as polymers and elastomers are lighter but lack the required strength and stiffness for high performance applications. Advanced composites materials, made from joining advanced fibers within a matrix material, provide a competitive material choice as 
compared to the mechanical properties of metals. Materials such as Glass Fiber Reinforced Plastics (GFRPs) weigh around 15\% that of steel and material costs are roughly on the same order of magnitude.

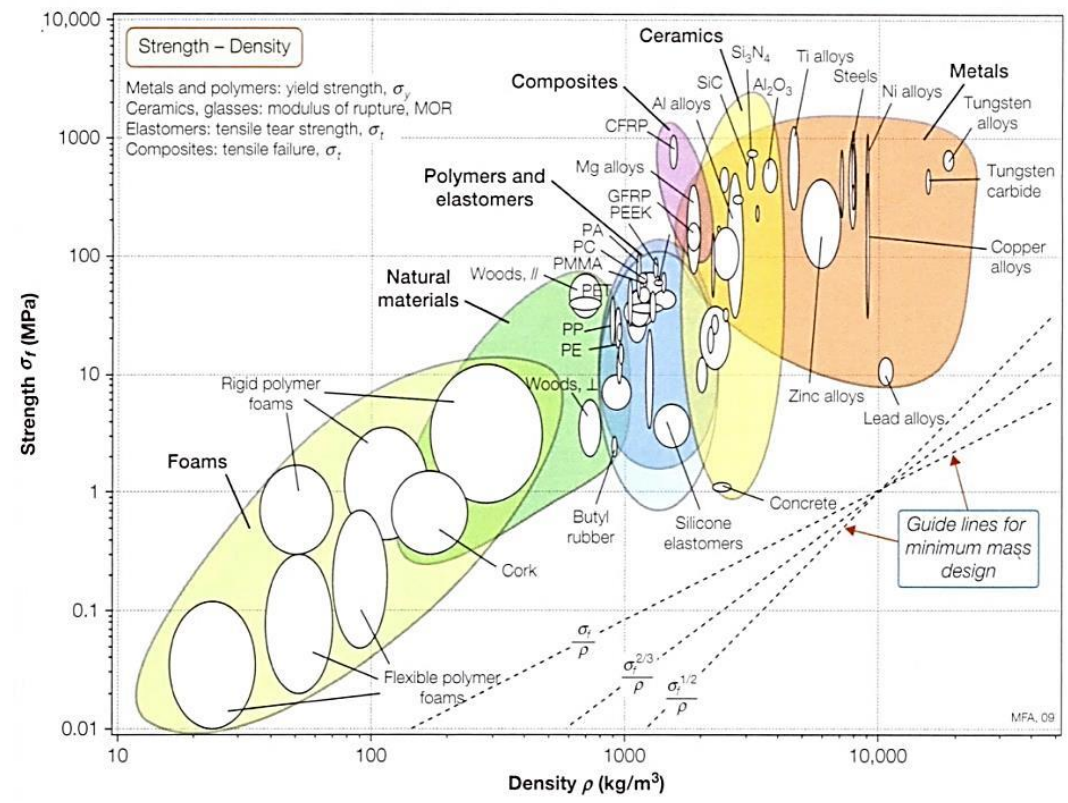

Figure 11: Strength plotted against density for structural materials (yield strength is plotted for metals and polymers, compressive strength for ceramics, and tensile strength for composites) [21].

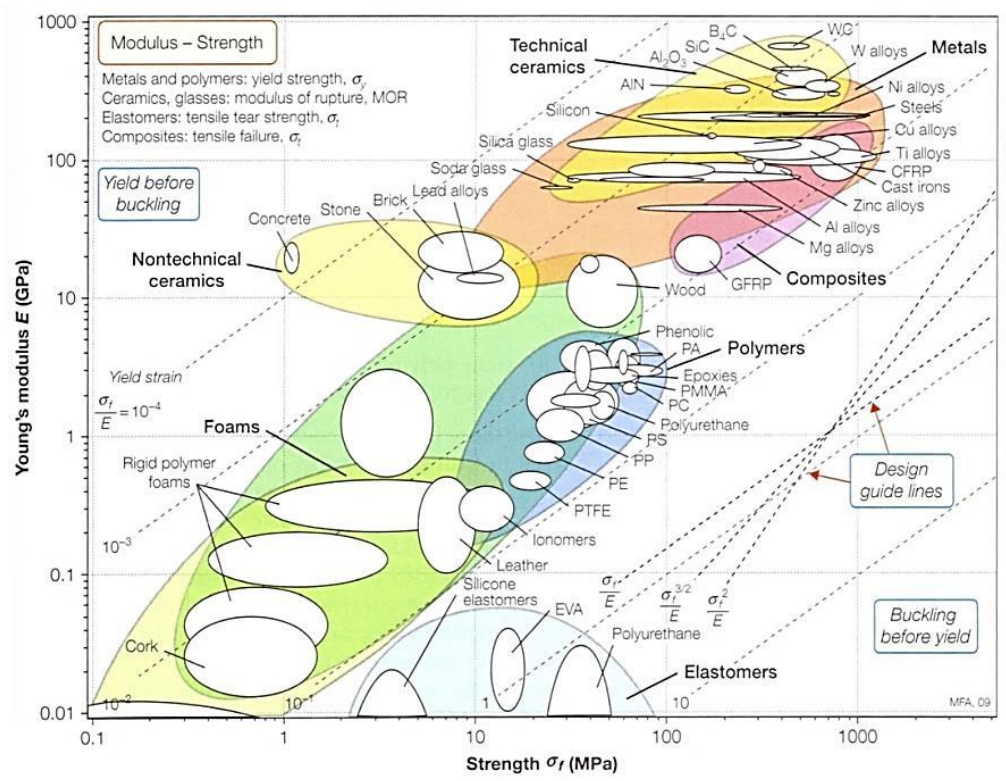

Figure 12: Young's modulus, E, plotted against failure strength [21]. 


\subsubsection{Fibers}

A majority of materials exhibit increases in strength and stiffness when a bulk material is manufactured in fiberous form. A high fiber aspect ratio (length/diameter ratio) permits a highly effective transfer of an applied load via the matrix material to the fiber, thereby taking advantage of the excellent properties of the fiber material [22]. Advanced fibers are manufactured from a variety of materials to include glass, carbon/graphite, polymers, and metals. Bulk metals commonly associated with structural applications and used in blast panels, including aluminum 6061, 4340 steel, 304L stainless steel, and titanium alloys offer specific strengths up to 0.3 $\mathrm{MNm} / \mathrm{kg}$ and specific moduli up to $35 \mathrm{MNm} / \mathrm{kg}$. When compared to the properties of advanced fibers, Figure 13, metals can only achieve 15-20\% the specific strength achievable by glass fibers and $8 \%$ the specific strength of UHMWPE. While the specific modulus of metals is comparable to glass fiber, metals only achieve $8-10 \%$ the specific moduli capability of carbon fibers. The small material space that metals occupy is shown by the small box in the lower left corner of Figure 13. 


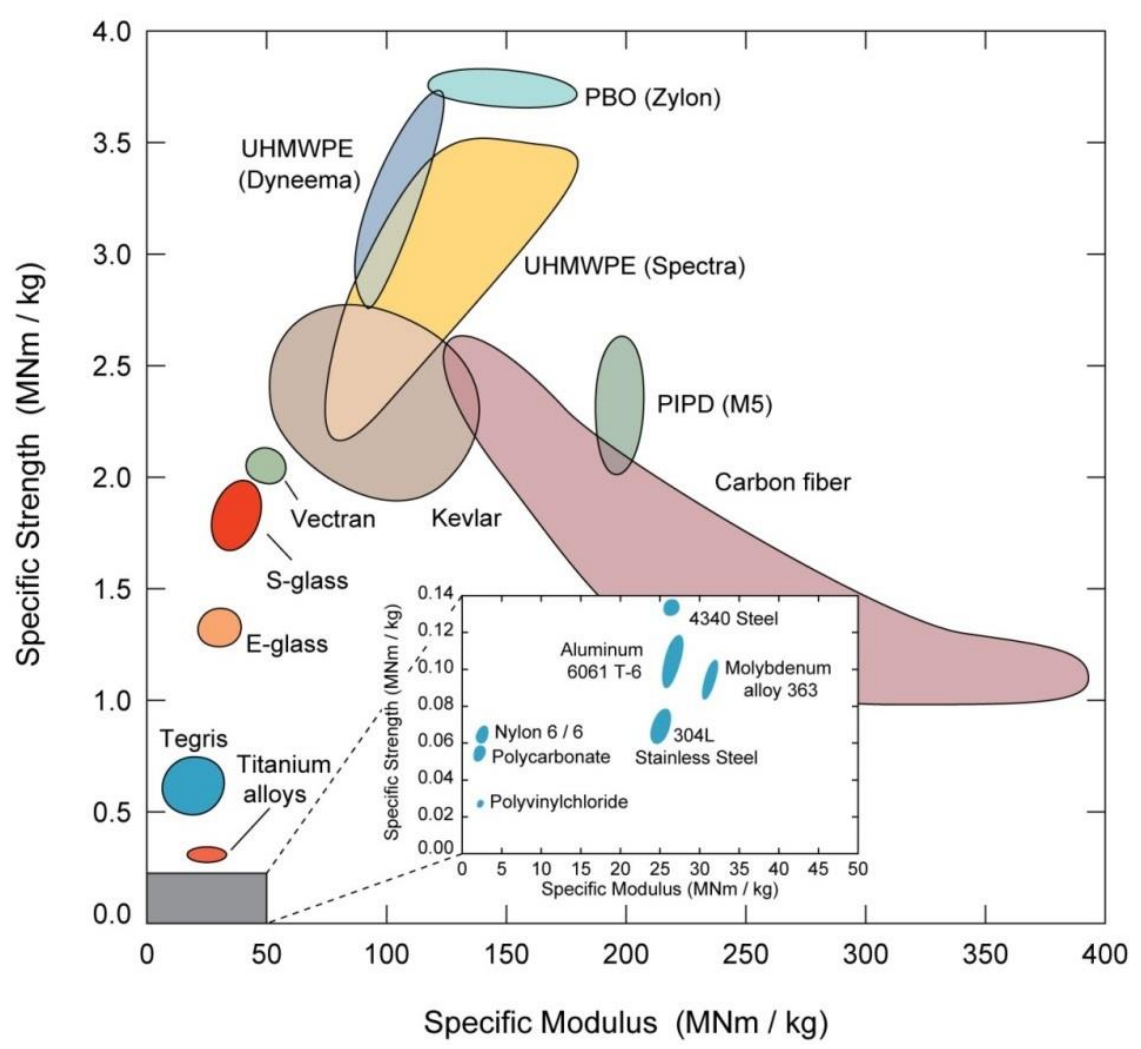

Figure 13: Specific tensile strength versus specific modulus for select metals, polymers, and composite fibers.

The mechanical properties of some of the most commercially used reinforcement fibers are shown in Table 1 for comparative purposes. Glass and polyester fibers are the most commonly produced and with the lowest associated manufacturing cost (1.7-3.9 \$/kg). For high tensile strength or higher stiffness demands, designers turn to carbon/graphite, aramid, or Ultra High Molecular Weight Polyethylene (UHMWPE) fibers. Fibers made from carbon, aramid, or UHMWPE often have properties that make them extremely difficult to process into a composite material as compared with glass fibers. Difficulty in handling, sensitivity to UV light, sensitivity to temperature, or inability/difficulty to bond with matrix material restricts the viability of these fibers in many applications. Glass fibers exhibit excellent manufacture ability and are the 
cheapest fibers available, making glass fiber the most used advanced fiber in composite materials.

Table 1: Mechanical properties of reinforcement fiber materials [22, 23]

\begin{tabular}{|c|c|c|c|c|c|}
\hline Fiber & $\begin{array}{c}\text { Density } \\
\left(\mathrm{g} / \mathrm{cm}^{3}\right)\end{array}$ & $\begin{array}{c}\text { Tensile } \\
\text { Strength } \\
(\mathrm{GPa})\end{array}$ & $\begin{array}{c}\text { Tensile } \\
\text { Modulus } \\
(\mathrm{GPa})\end{array}$ & $\begin{array}{c}\text { Ultimate } \\
\text { Tensile } \\
\text { Strain }(\%)\end{array}$ & $\begin{array}{c}\text { Cost } \\
(\$ / \mathrm{kg})\end{array}$ \\
\hline E-Glass & 2.54 & 3.5 & 72.4 & 4.8 & $1.7-2.7$ \\
S-Glass & 2.48 & 4.6 & 85.5 & 5.7 & 8.8 \\
$\begin{array}{c}\text { Aramid } \\
\text { (Kevlar 49) }\end{array}$ & 1.83 & $2.8-3.6$ & 130 & 2.9 & 35.3 \\
$\begin{array}{c}\text { UHMWPE } \\
\text { (Spectra 900) }\end{array}$ & 0.97 & 2.59 & 117 & 3.5 & 48.5 \\
$\begin{array}{c}\text { Polyester } \\
\text { (COMPET) }\end{array}$ & 1.36 & 1.03 & 9.6 & 22 & 3.9 \\
$\begin{array}{c}\text { Carbon-(PAN) } \\
\text { High Modulus }\end{array}$ & 1.90 & 2.1 & 390 & 0.38 & $37-1000$ \\
$\begin{array}{c}\text { Carbon-(PAN) } \\
\text { High Strength }\end{array}$ & 1.90 & 2.5 & 240 & 2 & $37-1000$ \\
\hline
\end{tabular}

Glass fibers are the most common of all reinforcing fibers for polymer matrix composites with the principal advantages being a low cost and high strength. Glass fibers can be produced by continuously drawing filaments through a melting \& refinement tank, but caution is required during manufacture. Glass fibers exhibit poor abrasion resistance and damage occurs that leads to the degradation in strength. In fact, strength values of a finished fiber may range from 50$75 \%$ of a virgin fiber and can continue to diminish through the further processing of the fiber. To aid in fiber protection and improve adhesion between the fiber and matrix, surface treatments on the fiber, known as sizing, are applied during manufacture. Most glass fibers are finished 
with a silane coupling agent/sizing that account for $0.1-0.5 \%$ of the weight of the fiber and provide superb bonding ability between the fiber and a polymer matrix [22].

The challenge of manufacturing a high strength composite material is capturing the material properties of the fiber into the composite material. The design of a composite material can be changed in many ways by altering the orientation of the fibers themselves. Unidirectional composites orient all fibers in a parallel direction to the axis of loading. Unidirectional composites exhibits the highest tensile strengths, but fail at significantly lower strengths when loaded off the axis of the fibers, which is highly dependent on the matrix material. A successful composite uses the matrix material to distribute the load onto a fiber and allows the fiber to bear the bulk of the load. As a result, many methods of composite fabric manufacture exist to include weaving, braiding, and knitting. As seen in Figure 14, the manufacture method can greatly alter the fiber orientation as function of position within the fabric. The resulting fabric design can greatly increase the effectiveness of a composite when loaded along varying directions as well as decrease the time needed for manufacture. The disadvantages include increase damage to the fibers during the manufacture process and design imperfections inherent to the fiber path created by a given fiber architecture. 


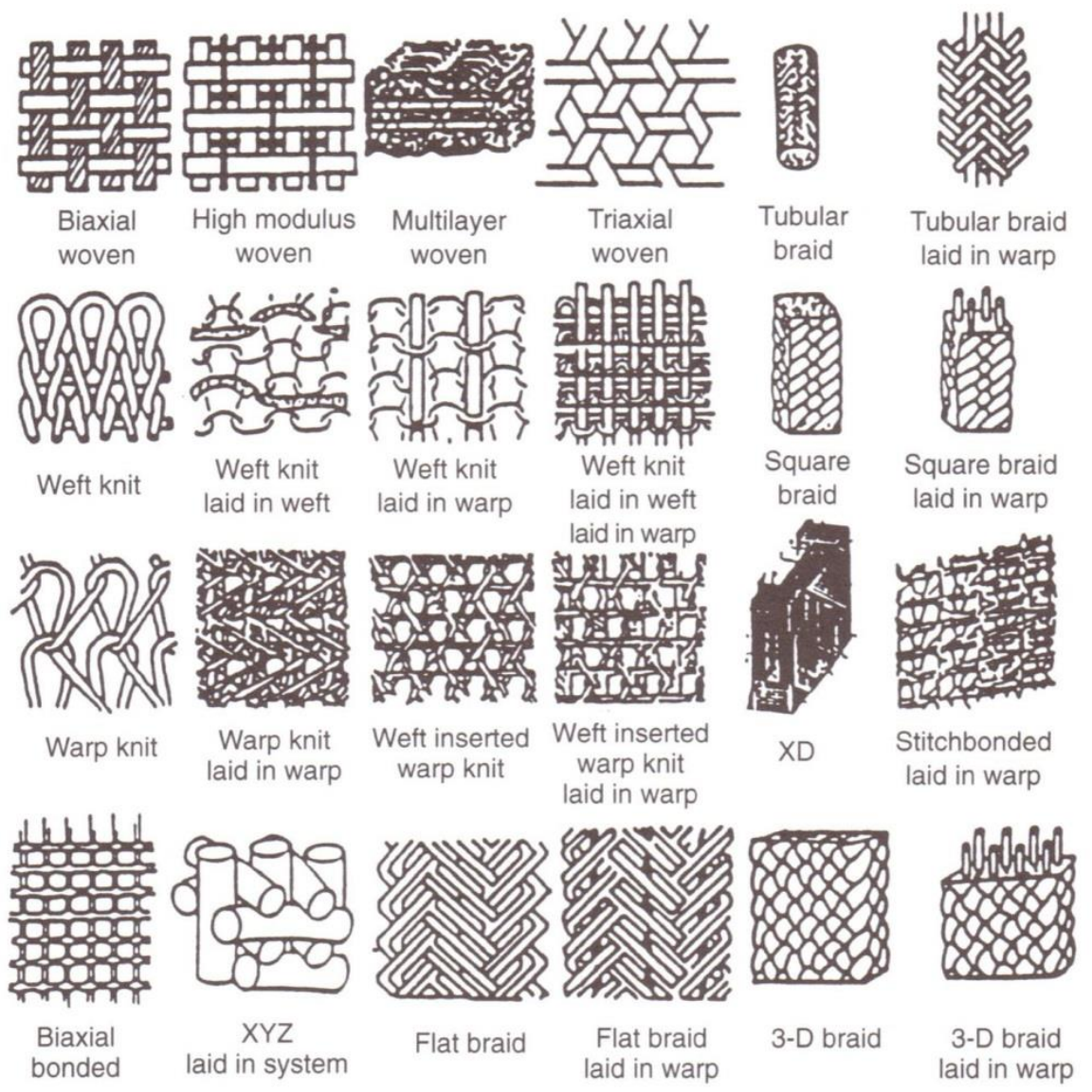

Figure 14: A comparison of yarn-to-fabric preforms including weave, braid, and knitting processes [24].

The majority of composites in existence today are built from either a unidirectional (1D) or two-dimensional (2D) fiber architecture pattern and laminated to create the necessary thickness for the composite material. The 2D patterns can easily be modified in design to provide various weave pattern which will vary the performance and appearance of the composite. 2D weaves inherently create imperfections by the misalignment of fiber tows during the weave process. Under a prescribed direction of loading, fiber tow misalignments are known to decrease the critical failure strength. However, a highly interwoven weave pattern will slow crack propagation that could be initiated from internal delamination. As a result, variations in the 
fiber architecture are continuously being investigated for a given loading situation for optimization. Figure 15 illustrates common variations in a 2D biaxial woven fabric. The fiber tows intersection positions within a plain wave can be adjusted be alternating the weave pattern to create a $2 \mathrm{D}$ fabric that overall increases in fiber tow straightness at a cost of fewer intersection positions.
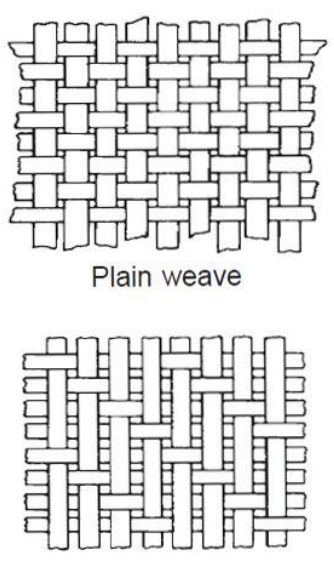

Crowfoot satin
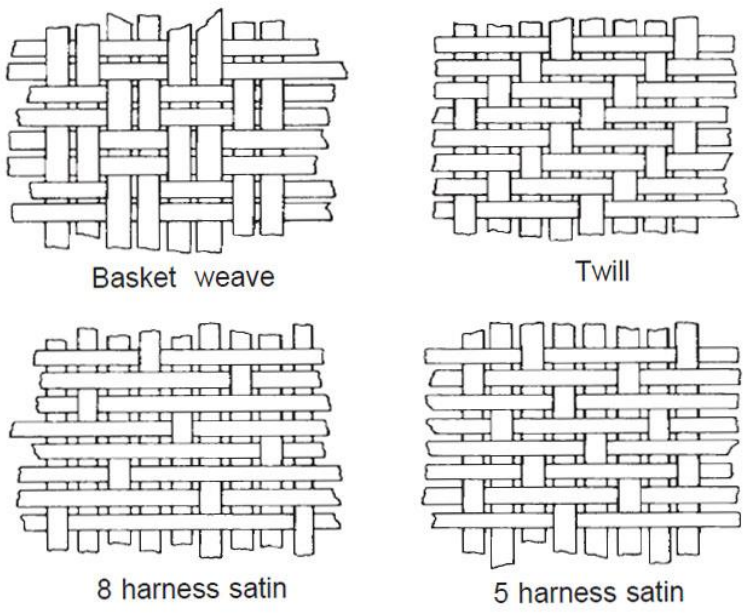

\section{Figure 15: Variations in a biaxial 2D woven fabric [23]}

An illustration of the fiber tow misalignment within a woven roving and knitted biaxial fiber architecture is shown in Figure 16. The end view of the woven roving show how the horizontal fiber tows vary in alignment along the length of the fiber tow. Variations in the fiber tow misalignment occur along both primary fiber tow axes and create localized positions of strain on a fiber/fiber tow that may artificially reduce the strength of the composite. An improvement in the fiber tow waviness is observed in the knitted biaxial fiber architecture where the primary fiber tows are allowed to remain straighter in their orientation. 

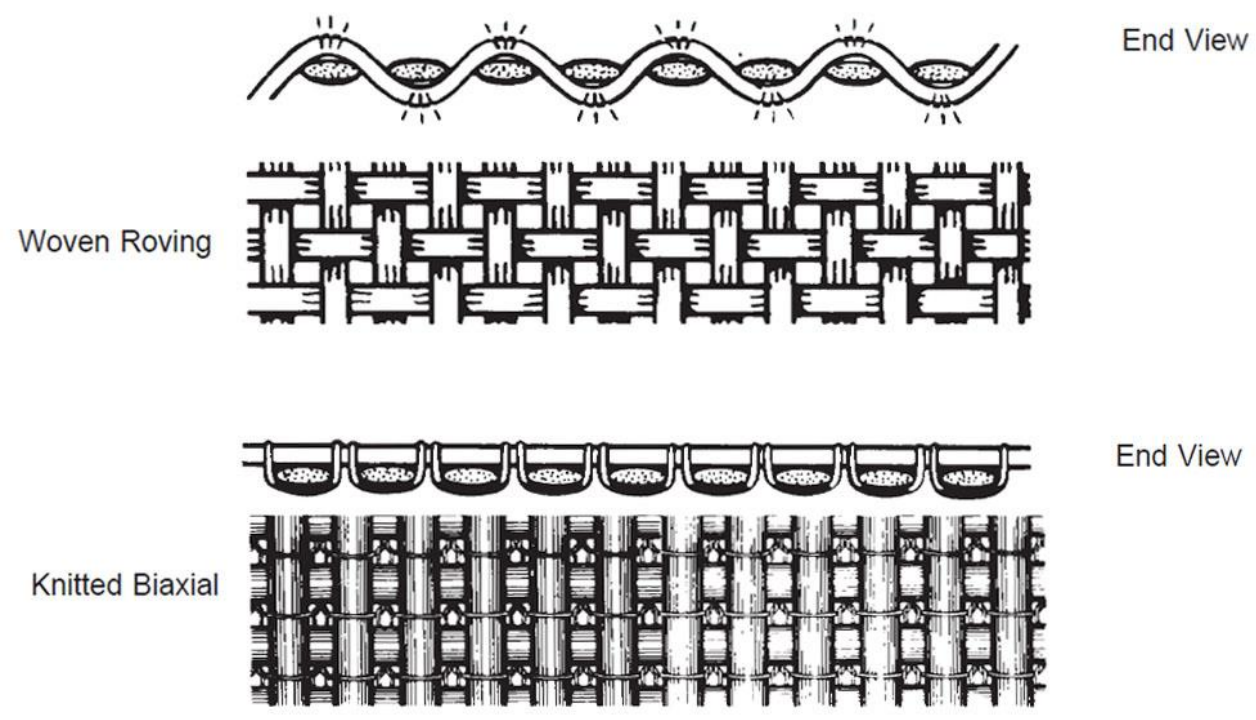

Figure 16: Comparison of a woven roving and knitted biaxial fiber architecture illustrate the fiber waviness/misalignment that is inherent to a given fiber architecture design [23].

Historically a great deal of research and analysis has been applied to investigating the tensile response of advanced fibers, fiber architectures, and laminated composites. Researchers generally agree on the basic analysis of tensile test methods because failure nearly always occurs by fiber or matrix tensile yielding. Little research has been done to investigate the compressive effects on this materials and designs. Further still, limited agreement is observed on how to predict the compressive failure strength due to an increase in observed failure mechanisms including delamination, brooming, fiber kinking and buckling, and fiber/matrix yield.

\subsubsection{Matrix Materials}

A matrix material is essentially the "glue" that holds the fibers together in a composite material. The matrix is essential to a high performance advanced composite in that it provides the mechanism to transfer load from one fiber to another as well as provide increased cross sectional area for loading and mounting purposes. The matrix also provides additional 
environmental protection to the fibers, needed for long term protection of the composite. The matrix has a strong influence on several mechanical properties of the composite such as the transverse strength and modulus, shear properties, and compressive properties [22].

Matrix materials for a composite are determined by the temperature region in which the composite will be used and the fibers in which they will be bonded together to form the composite. The most common matrix materials include polymers, metals, glass, ceramic, and carbon, as shown in Figure 17, as divided into their usable temperature ranges.

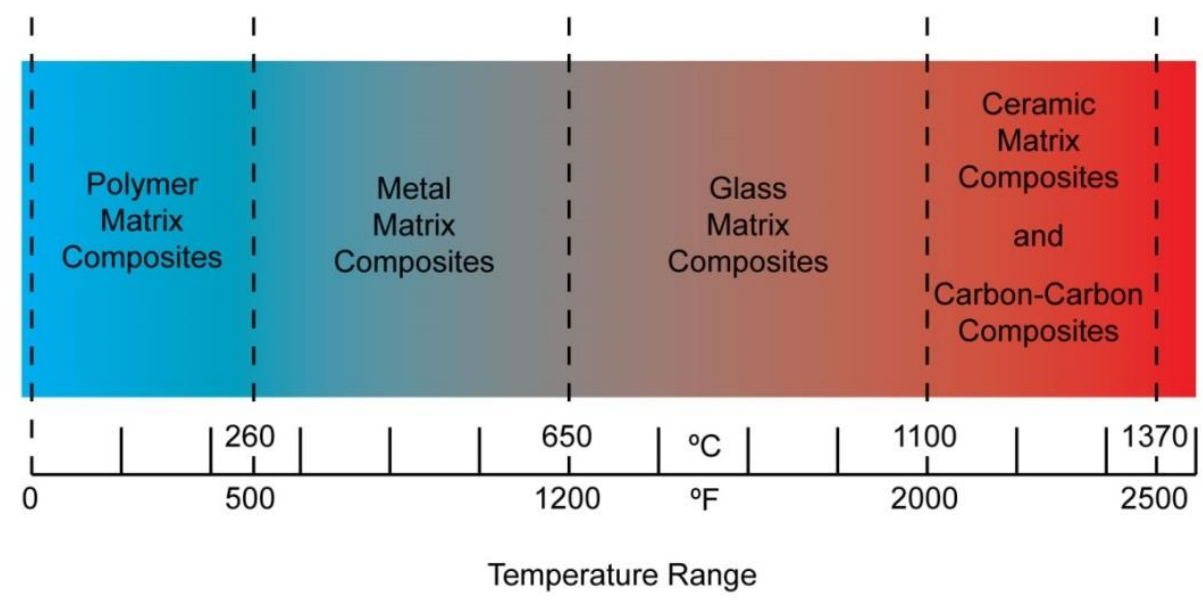

Figure 17: Usable temperature ranges for composites with different matrix materials [22].

Polymers are the most widely used matrix material for fiber composites and grouped into two main categories: thermoset resins and thermoplastic resins. The chief advantages of both polymer matrix groups are low cost, easy manufacture, good chemical resistance and low density. Polymer matrix materials are not, however, without their negative attributes to include low strength, low moduli, and sensitivity to UV light, solvents, and temperature. Polymer temperature sensitivity creates severe limitations critical to design. A thermoplastic polymer will form either a semicrystalline or amorphous microstructure while a thermosetting polymer 
generally exhibits only an amorphous microstructure. Thermoplastics with a semicrystalline microstructures exhibit a crystalline melting temperature $\left(T_{m}\right)$ while both polymers with an amorphous microstructure exhibits a glass transition temperature $\left(\mathrm{T}_{\mathrm{g}}\right)$, as shown in Figure 18. The glass transformation temperature provides an upper temperature limit on the effective use of the matrix material $[22,25]$.

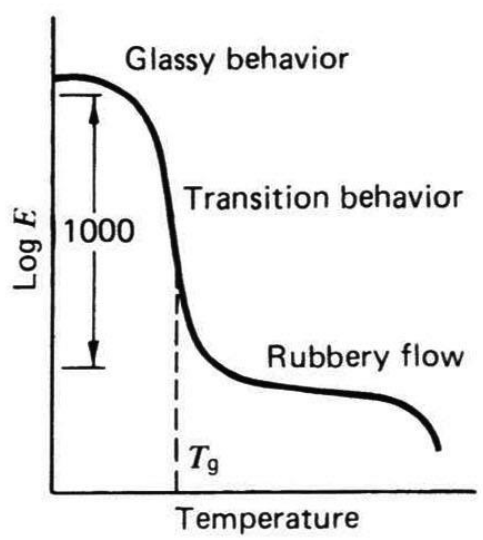

(a)

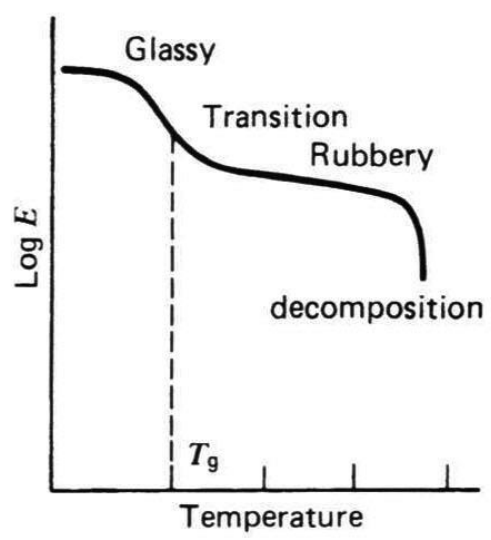

(b)

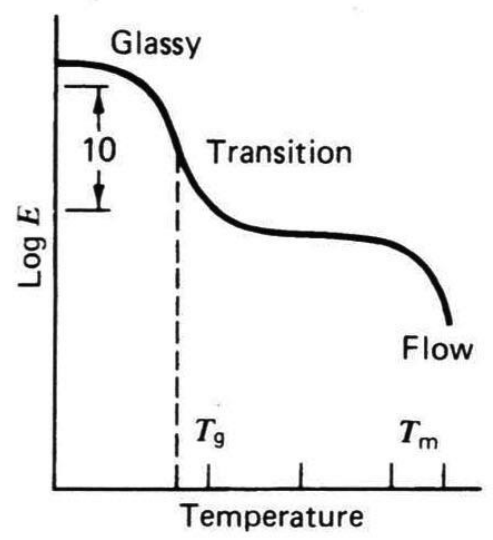

(c)

Figure 18: Variations in the elastic modulus of polymers with temperature: (a) thermoplastic amorphous, (b) thermoset amorphous, (c) thermoplastic semicrystalline [22].

\subsubsection{Manufacture Methods of Fiber Reinforced Polymer (FRP) Composites}

There are several manufacturing methods to create Fiber Reinforced Polymer (FRP) composites used in industry today. The size, complexity, and tolerances need for a composite component will often limit the manufacturing method required. The following is a brief overview of the main manufacturing methods used in industry today.

Hand Lay-Up Technique: The hand lay-up technique is the oldest, simplest, and most common method used to manufacture small and large reinforced composite components. A flat surface or mold is used to define the shape of a part and fiber reinforcements are manually 
placed in position against the mold surface. Resins are added to the reinforcements and often layers of material are added incrementally to create a laminated composite component. This method is an open-mold process and is often limited by the working time of the resin being used as the matrix material [22]. Hand lay-up techniques are best used in applications for low quantity or ease in part variability during production. The hand lay-up technique can use fiber mats or chopped fibers (often applied with a spray gun) to build the thickness of the part $[2,25]$. Parts made by this technique often have larger part-to-part variability in thickness dimensions, trapped air pockets, increased resin voids, and less than ideal fiber volume fractions due to the human factor.

Bag Molding Technique: The bag molding technique use a flexible membrane/bag to provide pressure to consolidate a resin infused part. The part is positioned on a flat surface or within a mold and the membrane/bag is placed over the part and sealed along the outer edges with a sealant tape or clamp system. Different methods exist to applying pressure to the composite lay-up to include a system of external pressure outside the part or a vacuum produced inside the membrane/bag. The net result is a pressure differential that is created the pressure outside the membrane is higher than inside, thereby applying pressure for consolidation and to maintain part placement during the curing process [2]. In some instances, the part is constructed initially through the hand lay-up technique and the bag is fitted after full matrix infusion. Other techniques use a Vacuum Infusion Process (VIP) to pull resin through the part after then entire dry fabric composite has been set in place and a vacuum initially pulled on the part [22]. One variation is the Close Cavity Bag Molding (CCBM) technique, which uses a preformed, reusable silicone bag to manufacture composite components. This technique uses a vacuum system to 
pull the resin through the part while maintaining part consistency through the preformed $\mathrm{bag} / \mathrm{membrane}$ system.

The advantages of this process include high strength parts through increased fiber volume fractions, increased part consistency, and higher part tolerances. This method is relatively inexpensive when used solely as a vacuum system, but can increase in cost when using autoclaves for pressure and temperature cure. In the case of leaks or too great of vacuum pressure, air pockets, resin voids, or resin vapor pockets can be created leading to imperfections in a composite part. Often pre-pregnated composite fabrics are used in this process to increase part consistency and decrease manufacture time [25].

Resin Transfer Molding: The Resin Transfer Molding (RTM) process uses a rigid mold to inject the resin matrix material into the part. The mold is often a two part mold designed to have the fibers placed between the mold surfaces to provide exact part dimensions. The dry fabric is fastened inside the rigid mold prior to sealing the mold pieces together. The resin matrix is introduced through a pressured injector head and resin flows through the part to air escape outlets. When the part is fully saturated with resin, the resin supply is removed the outlets are sealed. The composite part is cured inside the mold [2, 22].

Variations on the RTM process include a vacuum assisted method to move resin. Vacuum-Assisted Resin Transfer Molding (VARTM) starts with pulling a vacuum on the sealed mold to remove air. Resin is allowed to flow into the part and pulled through the part by the vacuum. In some cases, both vacuum and injector pressure are used to move the resin large distances. If only a vacuum is used, half of the mold may be replaced by a vacuum bag [22]. 
The RTM process provides better control over the orientation of the fibers, fiber volume fractions, and part imperfections overall creating a better composite material.

Filament Winding - The filament winding technique is reserved for the manufacture of surfaces around a mandrel, cylinder, or rotating shape. The process takes continuous fiber roving and wraps the fiber around a predefined shape. Often the mandrel is placed in rotation to draw the fibers in and maintain consistent tension on the fibers during the process. The fibers are sent through a resin bath prior to being wound around the mandrel. This technique can create hoop, helical, or longitudinal weave patterns around a mandrel and provides high fiber volume fractions capable of providing high strength components [22, 25]. 


\section{Chapter 3}

\section{Structural Systems}

Structural systems take advantage of the mechanical properties of a material when the material is positioned in a specific geometric orientation to provide support while under a loading condition. Structural systems are easily found everywhere in our world and occur both naturally and artificially at the microscopic and macroscopic levels. Natural structural systems often develop as a composite material incorporating a bulk material and a matrix material together naturally, such as wood and flesh. A bulk material used in a structure can be ideally homogenous, but often is heterogeneous due to impurities or design. Natural structures often occur in the form of a cellular structure, such as bone and coral, creating structures that are both strong and lightweight. Often the cellular structures within a natural structure are randomly or inconstantly built, exhibiting variations in cell size, position, and nodal attachment. Similar to foam, naturally created cellular structures often are dominated by bending failure of the cell walls. Artificial or man-made structures can be designed to alter the dominating failure mode from bending -dominated to stretch-dominated, thereby increasing the maximum strength of the structure. However, changing the dominant failure mode also changes the stiffness and energy absorption ability of the structure. To complicate the analysis, the failure mode often is dependent upon the rate of loading. 


\subsection{Cellular Structures}

Cellular structures are often used as the core within sandwich panel structures. Cellular structures are a class of ultralight materials made up of an interconnected network of solid struts or plates which form the edges or faces of cells [26]. The architectures of a cellular materials fall into one of two categories based on their morphology; open-cell and closed-cell structures [27, $28]$.

Open-cell structures contain pores that are connected to one another by the formation of a network of interconnected ligaments. There are no membranes sealing the developed porosity and fluid can flow throughout the structure. Close-cell structures have a similar network of interconnected ligaments but do not have interconnected pores. Rather, the pores have membrane walls enclosing the voids that prohibit fluid flow through the structure [29]. Open and closed-cell structures are shown in Figure 19. A cellular structure can be advantageous due to the associated decrease in material density while maintaining a load bearing capability and improving the energy absorption capability. Porosity can also provide methods for fluid flow through the structure and/or provide greater efficiency in heat transfer.
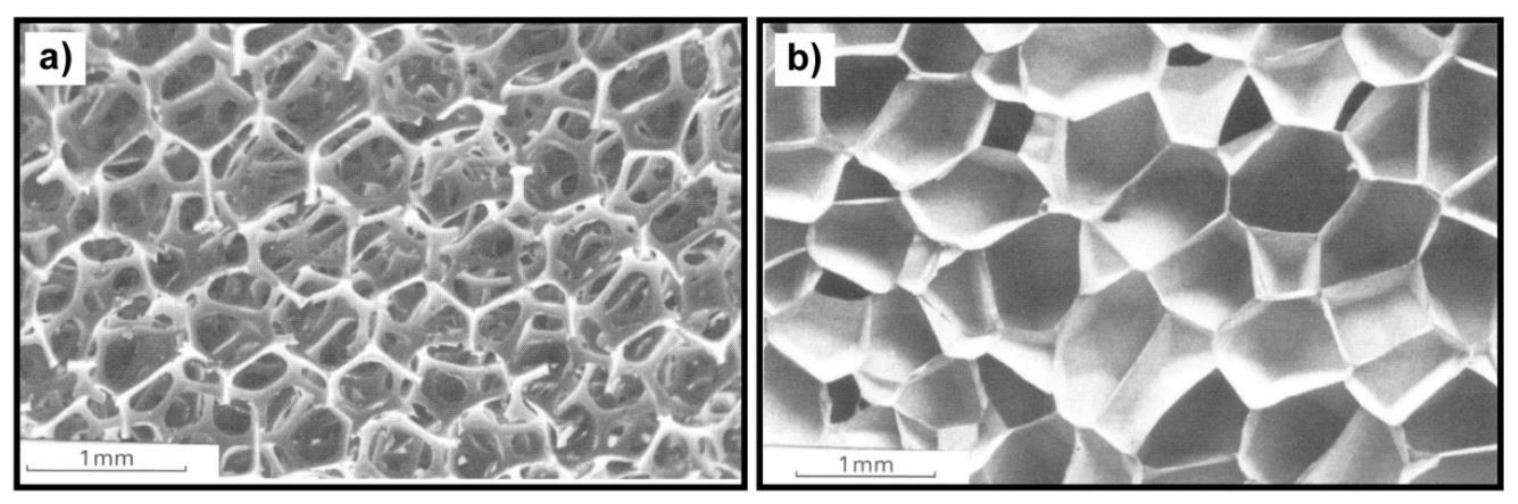

Figure 19: Examples of cellular structures morphology to include (a) open-cell and (b) closed cell structures. Both structures represent a stochastic cellular structure [30]. 
There are two primary types of cellular material structure; stochastic and periodic. Stochastic cellular structures possess a non-deterministic structural pattern, commonly associated with foams materials, while periodic cellular structures have a precisely designed structural pattern than maintains consistency in the cells geometric parameters and the cell is repeated throughout the structure.

Foam is a stochastic cellular structure that is formed by trapping pockets of gas within a solid or liquid material. The method of manufacture creates a structure where nodes, ligaments, and intended loading paths are not ideally positioned. When loaded, cellular wall failure initiates by buckling in a stochastic foam. Foams can be constructed from a variety of materials, metal and polymers as the most common foam materials, with relative densities ranging from $1 \%$ and upward [26]. These low relative density foams are an important class of cellular materials known as an ultralight material, i.e., a material with a density less than $1 \mathrm{~g} / \mathrm{cm}^{3}\left(1 \mathrm{Mg} / \mathrm{m}^{3}\right)$ [28]. Foam materials are displayed on Ashby’s material space maps, Figure 11.

The first synthetic analogues of random cellular materials were made by adding foaming agents to polymers [31]. Since then, foaming and other space holding concepts have been used to make cellular structures from metals [32] including aluminum [33], titanium [34, 35] and from ceramics [36]. Polymeric cellular materials are widely used for packaging to provide impact load mitigation [37] and energy absorption [38].

Polymer foams dominate the cellular materials industry with uses outside of structural applications to include packaging, buoyancy, and insulation because of their lightweight nature. The demand for higher strength core materials has led to the emergence of higher strength (although brittle) polymer foams, e.g., polyurethane, urethane-modified polyisocyanurate, and 
polyamide foams [39]. The continued interest in higher strength (and stiffness) foams led to numerous methods for making metallic and ceramic foams having various cell sizes and morphologies $[27,28,40]$. While stochastic foams are excellent energy absorbing structures, the mechanical strength is relatively poor compared to the theoretical upper (Voigt) bound for a structure made of solid and air (voids) [28].

The second primary type of cellular material structure is the periodic cellular structure. Exhibiting a higher degree of order in the design, a periodic cellular structure maintains consistency in the cell parameters to include shape, orientation, and size, along with the cell positioning within the structure. A cell pattern is repeated throughout the structure in a periodic fashion, as illustrated in Figure 20. Periodic cellular structures are designed to more optimally carry a load within the structure by geometric cell orientation and nodal positioning [28]. The design change can alter the primary failure mechanism from bending to stretch/yield thereby, increasing the critical failure strength and providing greater uniformity in performance.

Bending-dominated failure is geometrically determined, while stretch-dominated failure is determined by the bulk material [30].
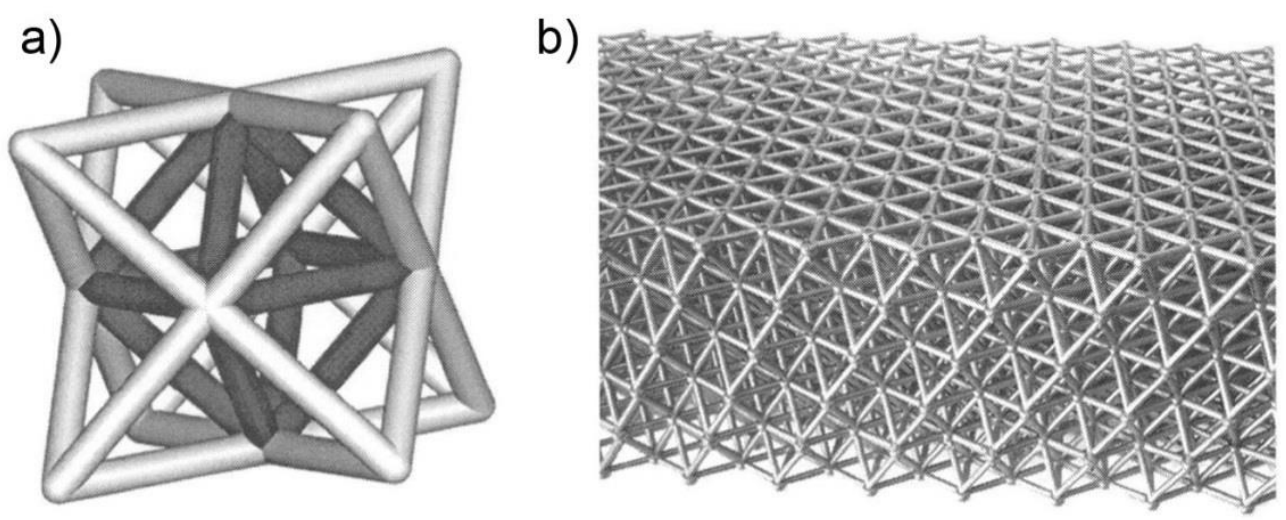

Figure 20: Stretch-dominated (a) unit cell and (b) lattice structure [30]. 
Manufacturing periodic cellular structures is often a challenge. Difficulty in maintaining the needed precise parameters and position of a cell can lead to the manufacture of panel with imperfections and alter the failure mechanism and significantly lower the mechanical properties. The majority of high strength periodic cellular structures are manufactured with metal bulk materials with isotropic materials. Methods have been developed for the fabrication of millimeter scale, open-cell metallic lattices from a range of light metals including high temperature alloys [41-49] and include designs such as honeycombs, tetrahedral, pyramidal, 3Dkagomé, diamond collinear, and square collinear lattice structures. Metallic pyramidal, tetrahedral, and kagomé structures are made predominantly by folding and brazing perforated metal sheets or by investment casting [41, 42, 44, 45, 50-54]. The collinear lattice structures exploit a wide range of metallic wire types and sizes for fabrication of cores by a lay-up and transient liquid bonding process [28, 46-49].

The overall strength of a periodic cellular structure is controlled by tensile, compressive, or shear strength of the lattice core material. Failure by stretching/yielding is governed by the bulk material strength while failure by bending/bucking is governed by the bulk material modulus [41-53]. To improve mechanical performance of a periodic cellular, it is necessary to utilize core materials with higher specific strength and stiffness. Composite materials offer a solution to the strength and stiffness, but add a difficulty in the manufacturing process. Carbon Reinforced Fiber Polymer (CFRP) is used to fabricate pyramidal lattice and square honeycomb cores [55-57] that are as strong and stiff as aluminum and titanium alloy lattice cores but are approximately 2-4 times lighter [28]. 


\subsection{Cellular Structure Failure Mechanisms}

The two primary types of cellular material structures, stochastic and periodic, fail by very different methods. The stochastic cellular structure is built in such a manner that macroscopic loading causes internal bending of the cell walls before material yield occurs. Periodic cellular structures are designed to place the cell walls under axial loads, thereby changing the failure mode to stretch-dominated ${ }^{2}$, creating a structure highly dependent upon the material properties.

\subsubsection{Bending-Dominated Structures}

A bending-dominated structure is one that responds, at the meso-structural level, by bending, buckling, and fracture of the cell walls, regardless of the direction of the applied macroscopic load [17]. Stochastic foams always exhibit this response as foams always have cell walls randomly oriented within the structure and, therefore, the cell wall interfaces are nonideally positioned. The result is a sufficient fraction of cell walls aligned at the most disadvantage angle with respected to the applied loading. Connecting cell walls or nodes will connect at the mid- length position of the neighboring cell wall/node transmitting the applied load in an non-axial direction $[17,28]$.

\footnotetext{
${ }^{2}$ The term stretch-dominated failure is synonymous with yielding under tensile or compressive loading.
} 


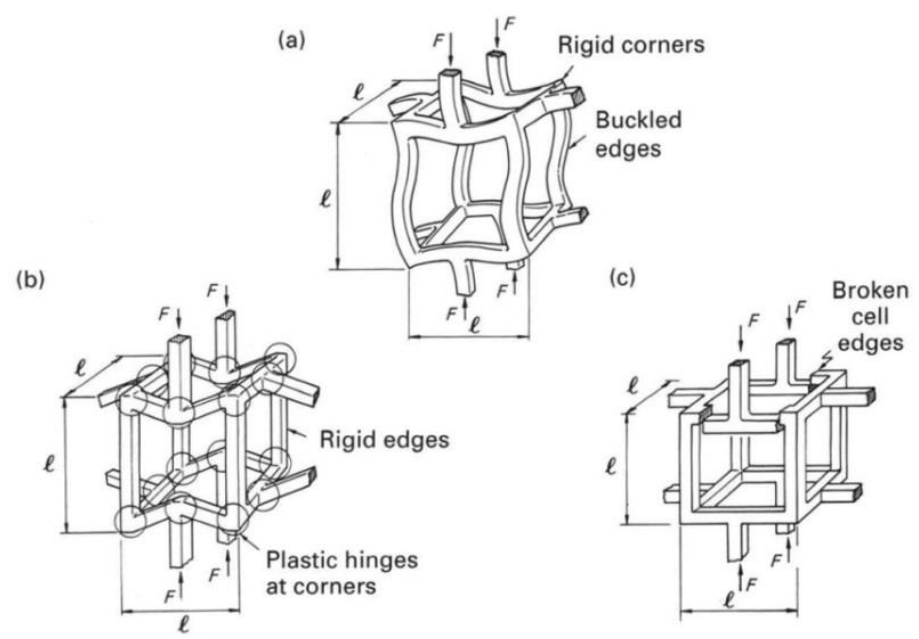

Figure 21: Bending-dominated structures fail by slightly different methods depending upon the material. (a) Ductile material causes cell edge bending. (b) Elastomeric materials cause cell edge buckling. (c) Brittle materials collapses by successive fracturing of cell edges $[26,28,30]$.

A typical compressive stress-strain curve of a bending-dominated cellular structure creates a plateau stress, $\sigma_{\mathrm{pl}}$, after the onset of bending failure. When loaded, the material is initially linear elastic up to the elastic limit. The cell edges or nodes will initiate failure by bending, buckling, or fracturing just past the elastic limit, Figure 21 . The material will continue to collapse at a nearly constant stress until the internal volume is reduced to the point where the cell walls impinge upon themselves, causing the stress to rise significantly [28]. The point in which a sharp change in stress-strain response occurs just prior to densification is known as the densification strain limit, $\varepsilon_{D}$. While bending-dominated structures are limited by the peak strength capability, the stress-strain characteristic makes foam materials attractive for energyabsorbing applications [17]. A typical stress-strain response for a bending-dominated structure is illustrated in Figure 22. 


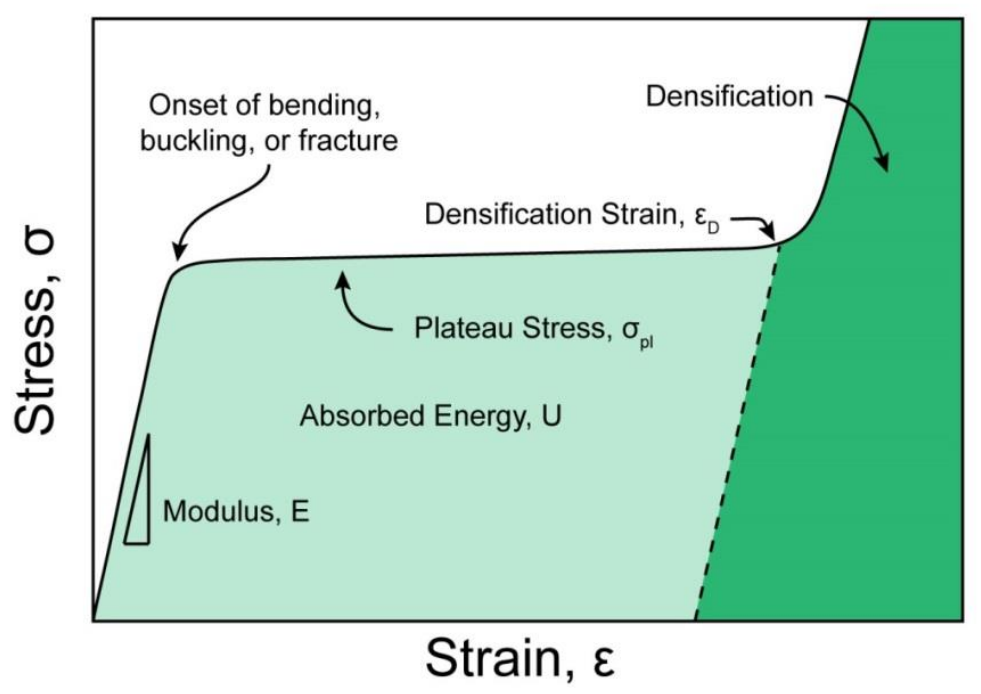

Figure 22: Classic stress-strain response of a bending-dominated cellular structure including both open and closed-cell stochastic foams.

Mechanical property scaling relations for bending-dominated foam materials have been established by Gibson and Ashby throughout many of their research works $[26,30,58]$. These scaling relations have become the standard in industry to determine the material properties of foam and are developed around the relative density of the foam as given by;

$$
\bar{\rho}=\frac{\rho^{*}}{\rho_{s}}
$$

where $\rho^{*}$ is the density of the foam and $\rho_{s}$ is the density of the bulk solid. The Young's

modulus of a bending-dominated structures can be estimated by the following relation;

$$
\frac{E^{*}}{E_{s}} \approx \bar{\rho}^{2}
$$


where $\mathrm{E}^{*}$ is the Young's modulus of the foam material, $E_{s}$ is the Young's modulus of the bulk solid. The shear modulus is estimated by;

$$
\frac{G^{*}}{E_{s}} \approx \frac{3}{8} \bar{\rho}^{2}
$$

Varying empirical relations were determined for the strength of the foam as dependent upon the precise method of bending-failure. If the foam collapses by plastic yielding of the cell edges, the failure strength can be expressed by;

$$
\frac{\sigma_{p l}^{*}}{\sigma_{y s}} \approx 0.3 \bar{\rho}^{3 / 2}
$$

where $\sigma_{p l}^{*}$ is the plastic yield strength of the foam, $\sigma_{y s}$ is the yield strength of the parent solid.

If the foam is an elastomeric and failure occurs by elastic buckling, the collapse strength is;

$$
\frac{\sigma_{e l}^{*}}{E_{s}} \approx 0.05 \bar{\rho}^{2}
$$

where $\sigma_{e l}^{*}$ is the elastic yield strength of the foam. If the foam is brittle and collapse occurs by cell wall fracture/crushing then the crushing stress of these foams can be estimated by;

$$
\frac{\sigma_{c r}^{*}}{\sigma_{f s}}=0.2 \bar{\rho}^{3 / 2}
$$

where $\sigma_{f s}$ is the modulus of rupture of the strut. The densification strain is used to estimate the energy absorption of a bending-dominated structure. The densification strain is given by;

$$
\varepsilon_{D}=1-1.4 \bar{\rho}
$$




\subsubsection{Stretch-Dominated Structures}

A stretch-dominated structure is designed to load the structure's ligaments in a predominantly tensile or compressive axial stress [17]. The ligaments are intended to uniformly support the applied stress, Figure 23, and as a result, stretch-dominated structures are much stiffer and stronger than bending-dominated structures of the same material and relative density [28]. The drawback to a stretch dominated structure is post-yield softening as seen in Figure 24 which can dramatically reduce the energy-absorbing ability.

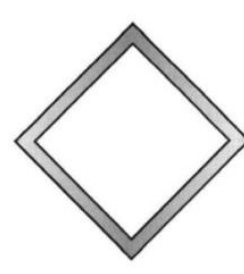

(a)

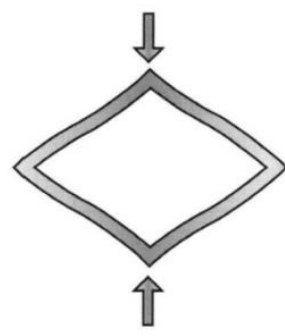

(b)

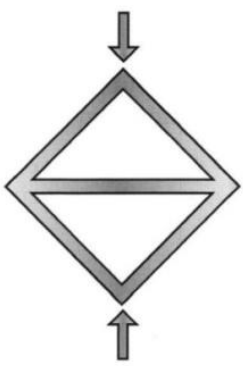

(c)

Figure 23: A stretch-dominated structure is stiff when stressed because loads are transmitted axially along the ligaments. For comparisons a diamond cell is (a) unloaded, (b) loaded without a transverse stiffener, and (c) loaded with a transverse stiffener to create a stretch-dominated structure [30].

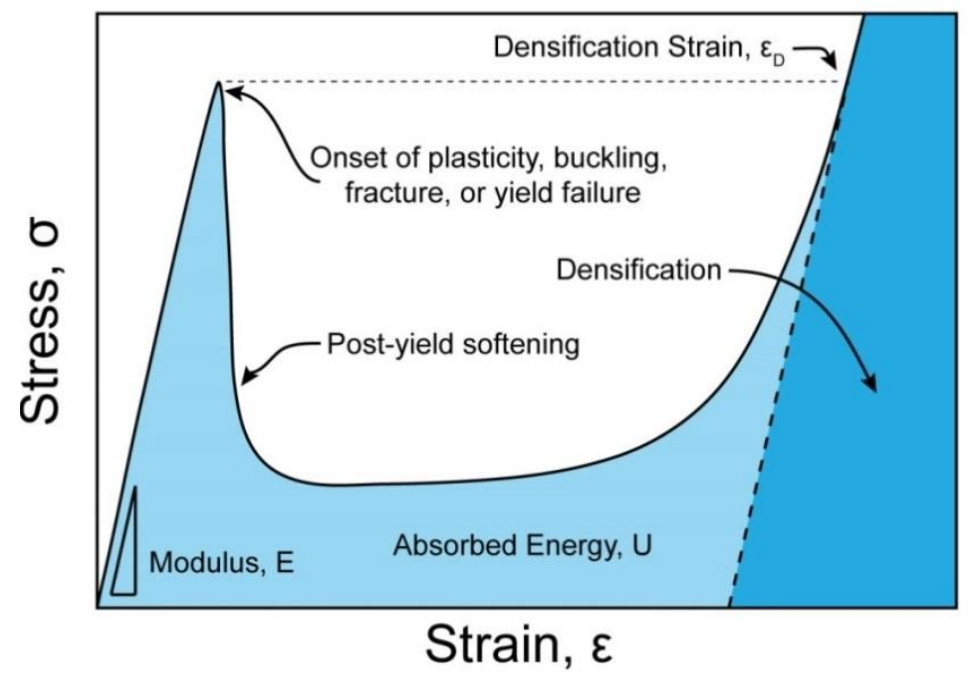

Figure 24: Classic stress-strain response of a stretch-dominated cellular structure. 
Similarly, mechanical property scaling relations for stretch-dominated structures, including honeycombs, prismatic and lattice structures, have been established by Gibson and Ashby [26, 30, 58]. When loaded, the structures ligaments first respond by elastic stretching in tension or compression, resulting in an effective Young's modulus of the cellular structure that scales linearly with the relative density as given by;

$$
\frac{E^{*}}{E_{s}} \approx \frac{1}{3} \bar{\rho}
$$

The elastic limit of stretch-dominated structures is reached when one or more sets of ligaments yield plastically, elastically buckles due to axial loading, or fractures. The failure mechanism requiring the lowest collapse load determines the overall failure strength of the structure [17]. In general, if the struts or cell walls are plastic and the structure's relative density is high, the structure will fail by plastic yielding with a the collapse strength given by;

$$
\frac{\sigma_{y}^{*}}{\sigma_{y s}} \approx \frac{1}{3} \bar{\rho}
$$

where, $\sigma_{y}^{*}$ is the structures yield strength and $\sigma_{y s}$ is the yield strength of the parent material [59].

When the relative density of the cellular structure is low exhibiting long, thin cell walls, ligaments or trusses, failure occurs by elastic buckling under compressive loading [59]. The overall compressive strength $\sigma_{e l}^{*}$ is then governed by the elastic buckling strength of such trusses or cell walls, $\sigma_{e l}$, and the structure behaves similar to buckling foams. Gibson and Ashby report varying values for the constant of proportionality, $\mathrm{C}_{1}$, ranging from 0.05 to $0.2[26,30]$. The magnitude difference in the constant of proportionality depends on buckling which depends 
on the slenderness ratio, $\mathrm{t} / \mathrm{L}$, of the ligament/truss. Ashby observed that, elastomeric foams always fail by buckling when $\bar{\rho}<0.05$, and rigid polymer \& metallic foams buckle before yield when $\bar{\rho}<0.01[17]$.

$$
\frac{\sigma_{e l}^{*}}{E_{s}} \approx C_{1} \bar{\rho}^{2} .
$$

Failure by strut fracture, in the case of ceramic or other brittle solid cell walls will cause the structure to collapse. Similarly to the plastic-stretch dominated failure, Equation 3.9, the tensile stretch-fracture dominated behavior is linear related to the relative density. If the trusses in tension fail first then the collapse strength is giving by Ashby [17];

$$
\frac{\sigma_{c r}^{*}}{\sigma_{c r, s}^{*}} \propto C_{2} \bar{\rho},
$$

where $\sigma_{c r}^{*}$ is the tensile fracture strength of the structure and $\sigma_{c r, s}^{*}$ is the tensile fracture strength of the bulk solid. Here, the constant of proportionality, $\mathrm{C}_{2}$, is less certain because brittle fracture is a stochastic process, which depends on the presence and distribution of defects in the trusses [28]. If however, the structure is placed in compression, the compressive strength is controlled by buckling stress as presented in Equation 3.10.

\subsection{Sandwich Panel Structures}

A sandwich panel structure consists of three main parts; a core and two outer edge facesheets, Figure 25. The facesheets are positioned parallel to one another and adhered to the core to provide a method to distribute loading among the core. The formation of a sandwich 
panel structure is the optimal way to take advantage of the mechanical properties of a cellular structure as the facesheets will bear loads, redistribute loads, and provide nodal boundary conditions to the core. Cellular material sandwich panels made of thin, stiff, and strong facesheets, are well suited for compressive and bending applications. As an alternative to solid materials, sandwich panel structures are widely used in weight sensitive applications such as aircraft, sea and ground transportation structures, wind-mills, packaging, and in sports equipment such as skis $[16,60,61]$.
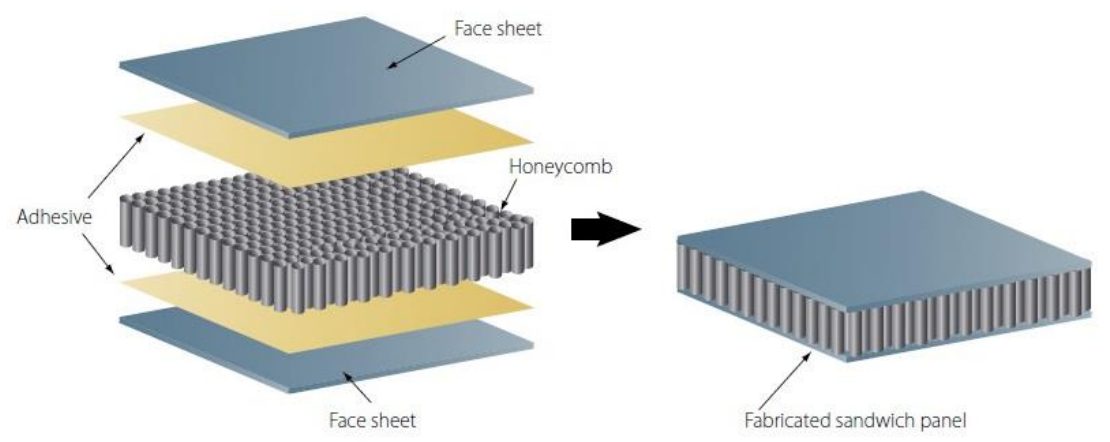

Figure 25: A sandwich panel structure is created by attaching a core (such as a honeycomb cellular structure as shown) to facesheet [62].

The use of structurally efficient sandwich panels is widely found in nature as well. For example, the human skull is made up of two layers of dense, compact bone separated by a lightweight core of spongy, cancellous bone. The beak and wing of a bird and the leaves, stalks, and the stem of plants also show a low-density foam-like core separated by solid faces. Besides supporting loads, these natural sandwiches have other functions. For example, while plant stems support the leaves, flowers, and fruits, the stems also transport water, mineral, and nutrients to leaves and roots. In cacti, additional functions of the stem are to store water and photosynthesis [28]. Most often the sandwich panels found in nature are stochastic. 
Periodic cellular structure sandwich panels have been developed with open-cell architectures [41-53], to offer new combinations of structural performance and multifunctionality. Numerous cell topologies have been created, including honeycomb [59, 63-72], corrugations[37, 73], tetrahedral [41, 50-53], pyramidal [42-44, 50, 52], 3D-Kagome [41, 45], woven textile [46-48], diamond collinear [49], and square collinear [49] structures, as seen in Figure 26 [28].

(a) Honeycomb (square)

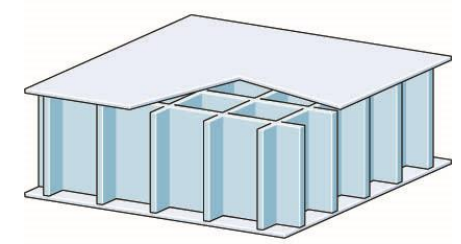

(d) Tetrahedral

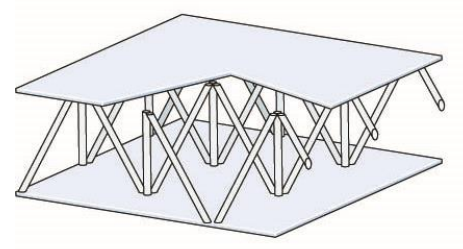

(g) Square Textile

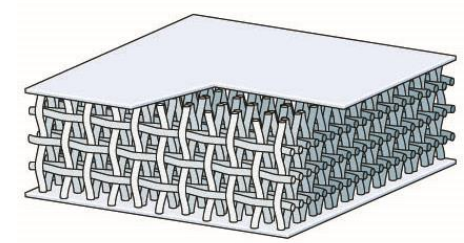

(b) Corrugation

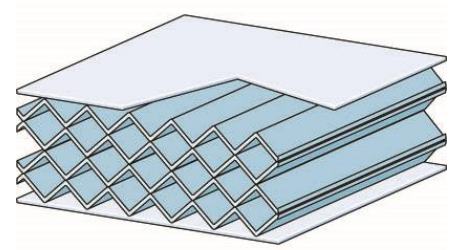

(e) 3D-Kagomé

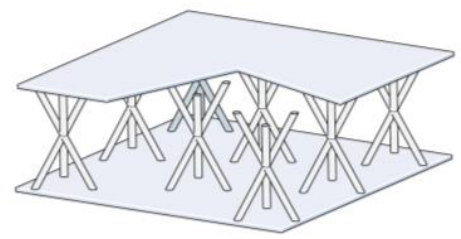

(h) Diamond Collinear

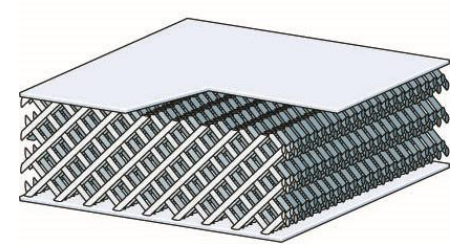

(c) Pyramidal

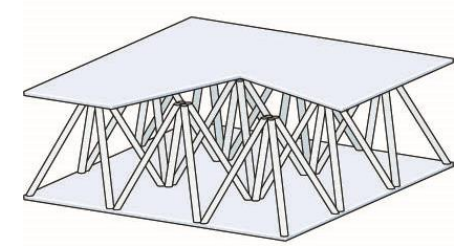

(f) Diamond Textile

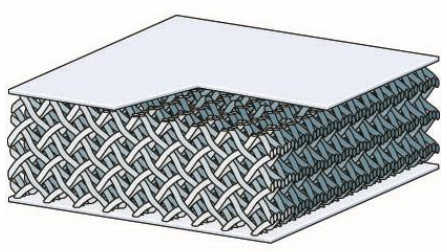

(i) Square Collinear

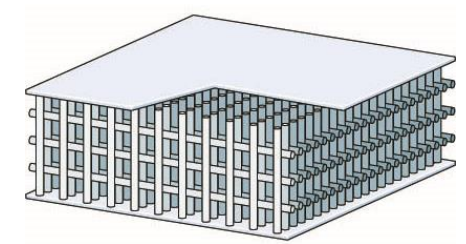

Figure 26: Examples of sandwich panel structures with various core topologies [28, 52, 59]

Truss members of periodic cellular materials are topologically configured to experience predominantly axial stress (tension and compression) when sandwich panels are loaded in 
compression or bending $[72,74,75]$. This results of these structures show significantly superior structural performance compared to stochastic foams whose ligaments experience predominantly bending when loaded [17, 28, 59].

The primary benefit of a sandwich structure is the ratio of strength and stiffness to the density under bending applications. The separation of the faces by the core increases the second area moment of inertia and therefore the section modulus of the panel with little increase in weight if a low density cores is used $[60,61]$. Since the faces carry most of the tensile load, they must have a high in-plane stiffness and strength $[60,61]$. The core occupies most of the volume of the structure so a lightweight and sufficiently stiff and strong core will be ideal. The core will carry the stresses necessary to ensure that the whole structure behaves as a load bearing unit [60, 61]. The core must keep the facesheets the appropriate length separation apart. If the core sufficiently crushes and allows the facesheet separation distance to diminish, the bending resistance will diminish [28].

A sandwich structure may not always be intended for use in bending applications. Often the advantages of sandwich structure are combined with a cellular structure core to provide purely through-thickness compressive resistance or energy absorption. Due to real-world loading imperfections, an applied load might be unequally distributed creating high stresses on localized regions of a core. Localized cell failure can lead to full core failure under the right scenario. As a result, a strong, stiff facesheet can be added to the core to provide a load distribution platform. This distribution requirement is often important in packaging and high load applications [16]. Additionally, the facesheet is advantageous to some cellular cores for nodal positioning and resistance to movement to interface cells of a cellular core. Cores such as 
corrugations, pyramidal, and tetrahedral often rely on a strong bond between interface cells and a facesheet to maximize the critical strength.

A sandwich structure core can serve a number of other roles outside of a purely loading function. Sandwich cores may provide multifunctional capabilities such as cross flow heat exchange [27, 59, 76-78], dynamic load protection [27, 59, 79-81], and acoustic damping [8284]. The facesheets serve a critical role to the overall protection of the core as the facesheets always will be the exterior surfaces of the sandwich structure. The facesheets must tolerate the environment in which they operate to successfully survive to provide structural performance. The structural performance and multi-functionality of sandwich structures depend greatly on the topology of the cellular material core, as well as on the intrinsic properties of the solid from which they are made $[60,61]$.

\subsection{High Explosive Blast on Sandwich Panel Designs}

Some classic cellular structure sandwich panel designs have been investigated under high explosive blast loading with the goal of finding structures that are viable as a blast protection armor or investigate the response to aid in the design of such an armor. Cellular structure designs including stochastic foams [85], corrugations [19], honeycomb [9], and pyramidal [14, 20], and have been investigated with metal materials including aluminum and steel. Restrictions on work performed with high explosives or limits in test size have led some researchers to investigate dynamic responses in unique manners. The use of high speed projectiles made from bulk $[37,86]$ or foam projectiles $[87,88]$ and underwater impulsive loading $[11,89]$ provide methods to evaluate the dynamic failure modes. 
Test methods and experimental conditions vary significantly throughout research studies. Edge-clamped high explosive blast analysis techniques are one of the most utilized test methods to investigate a structures failure mechanisms and response. However, the boundary condition mechanisms create loading conditions that artificially damages the panel due the clamping loads, blast reflection of mounting plates, and material stretching in localized areas leading to premature failure. Impulse measurements have been taken from vertical pendulum rigs [10], horizontal pendulum rigs [85], and structures affixed to pressure measurement devices such as Kolsky Bars $[37,86]$. Critical to the study of a structure is the investigation of effects of the test parameters. The results of these tangential studies indicate that parameters such as soil type, moisture content [90], depth of burial, charge initiation methods, and explosive material [10] greatly affect the energy transfer from the explosive to the soil and soil to the structure.

Analysis of the response of metallic, edge-clamped plates to localized impulsive loads led to a recognition that the out-of-plane deflection of the plate varies inversely with a material property index $\left(\sigma_{y} / \rho\right)^{1 / 2}$ where $\sigma_{y}$ is the yield strength of the material and $\rho$ its density [91]. Initial failure modes are often difficult to determine experimentally, but success has been obtained through the combined analysis of quasi-static and dynamic experimentation. Performing quasi-static tests illuminate bending or stretch-dominated failure to include core bending, buckling, fracture, yield, delamination, shear, and so forth. Under high strain rates, the geometric dependent failure modes are temporarily non-active and most structures behave as a stretch-dominated structure. Depending upon the strain rate, the constitutive materials of the structure may not be able to transmit the load quickly enough along a loading member. The result is a temporary condition where bending failure mechanisms are non-active and a structure may exhibit a 2 to 3 times increase in loading strength [37]. While high explosive blast events 
occur over a short time, the dispersed soil ejecta is sufficiently spaced to ultimately allow for slower strain-rates to occur and many secondary failure mechanisms to occur within a structure.

A cheap and effective solution for blast armor is continually being investigated for the military needs of tomorrow. Foam core sandwich structures offer a solution, but significant improvements are needed to effectively protect from an increased threat size. The recent development of much stronger metallic cellular materials with periodic (lattice) cell topologies [92] made from high strength alloys $[59,93]$ has led to considerable interest in their use for mitigating impulsive loads created by impacts [94] and interactions with shock fronts resulting from nearby explosions in water $[9,89,95]$, air $[9,96]$, and buried under soil $[15,97]$. The use of advanced composite materials within a cellular structure may provide a solution beyond the limits of metallic cellular structures. With specific strengths of advanced fibers much greater than metallic alloys, the leveraging of fiber type and orientation may provide a superior solution to localized impulsive load mitigation. 


\section{Chapter 4}

\section{Structure Design and Material Selection}

Research presented by Latourte et al. [11] presented the use of a PVC closed-cell foam sandwich structure when subjected to underwater impulsive loads. This structure uses an Eglass/vinyl ester composite for the facesheets with Divinycell H250 foam core. The results showed that under a specific applied impulse, the normalized peak deflection of the sandwich panel was less than the composite solid panel comparison and both delamination and fiber fracture failure within the solid composite and sandwich panel were observed as primary failure mechanisms. After observing the structure response, one of the most interesting aspects of this study was the materials used. This structure used some of the lowest cost materials in the advanced composite industry, E-glass fibers, vinyl ester resin, and PVC foam, and the panel responded quite well. Building from the concept of a low-cost glass fiber, PVC foam sandwich panels for impulsive load mitigation, a design for a lightweight, energy absorbing, hybrid glassfiber composite sandwich structure emerged.

Periodic cellular structures have shown the ability to increase the critical failure strength while maintaining the ability to absorb energy. While many periodic cellular structures have been investigated, corrugations were naturally of interest due to the ease of manufacture 
adaptability to composite fabrics. Aluminum corrugated cellular structures of a similar design were already used to study edge-clamped boundary condition high explosive blast loading and illustrated that weaknesses in the panel were partially due to material choice [19]. The aluminum corrugated core was developed with an inclination angle of $60^{\circ}$ for the core strut orientation [15, 19], and that design parameter is mirrored in this study. Development of a delamination resistant composite weave was underway by researchers at 3Tex, Inc. for a multi-function purpose of load bearing and armor protection ability. Research showing the shock loading [98] and ballistic resistance [99] of this 3D woven material showed excellent improvement in delamination resistance and a viable material for high explosive blast loading.

The design solution being investigated is a hybrid sandwich structure that utilizes corrugated composite cores constructed from delamination resistant 3D woven E-glass fiber textiles with a PVC foam placed in the voids of the corrugation. Strong, 3D woven S2-glass fiber facesheets are stitched to the corrugated core and infiltrated with a rubber toughened, impact resistant epoxy.

\subsection{Hybrid Corrugated Sandwich Panel Design}

A glass fiber composite sandwich structure integrating a corrugated composite/polymer foam core was chosen for study because; $(i)$ it is relatively simple to manufacture over a wide range of length scales, (ii) the high in-plane stretch and shear resistance and through-thickness (out-of-plane) compressive strength of the structure enhances the performance of heavily loaded edge clamped sandwich beams, and (iii) the closed cell foam confines resin infiltration within the glass fiber struts/faces. 
A PVC closed cell foam, Divinycell H130, was used for the core due to the low density and good energy absorption capability. To avoid interplay delamination [100], a composite laminate failure commonly seen during edgewise compression, an inexpensive 3D woven Eglass fiber architecture is used for reinforcement of the foam core. Similarly, to avoid delamination failure, a 3D woven fiber architecture was chosen, but made from higher strength S2-glass to increase the load carrying capability under bending deflections [22]. High strength Kevlar $^{\circledR} 29$ was used to join the facesheets to the corrugated core through a stitching process. Finally, the structure is entirely infused with a rubber-toughened ballistic certified epoxy to provide shock loading resistance to the structure. This study investigates the effects on mechanical performance when the relative density of the structure is varied by altering the strut slenderness ratio $(t / l)$, where $t$ is the strut thickness and $l$ is the strut length. A schematic illustration of the hybrid foam glass-epoxy corrugated cellular structure sandwich panel concept is shown in Figure 27.

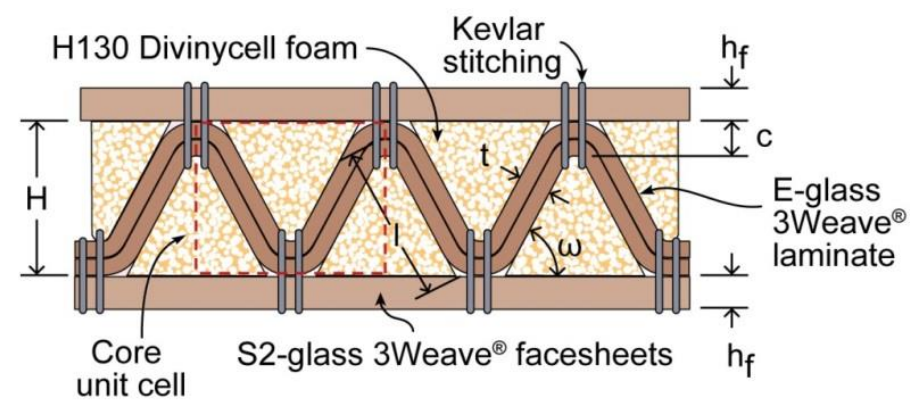

Figure 27: Design parameters associated with the hybrid foam glass-epoxy corrugated composite celluar structure sandwich panel.

The relative density of the structures core varied from $15-40 \%$ by changing the composite strut slenderness ratio ( $\mathrm{t} / \mathrm{l})$. Ultimately, four core densities were made with $\mathrm{t} / \mathrm{l}$ ratios ranging from 0.07 to 0.25 by the processes of laminating the E-glass fabric. While the design 
intent was to completely remove the lamination process in the core to remove delamination as a failure mode, lamination became essential to properly fold and manufacture the desired core (discussed further in Chapter 5). Core struts were constructed with 1, 2, or 3 laminates of 3D woven fabric resulting in webs with dry thicknesses of $1.49,2.98$, and $4.47 \mathrm{~mm}$. additionally; the relative density was varied by altering the strut lengths in the sample. Strut lengths of $14 \pm 1 \mathrm{~mm}$ and $25 \pm 1.5 \mathrm{~mm}$ where used as the only length options. The strut thickness $(\mathrm{t})$, core height $(\mathrm{H})$, node thickness (c), strut length (1), and strut orientation angle $(\omega)$ define a core design, Figure 27, and the values for the four designs are summarized in Table 2. The facesheet thickness $\left(\mathrm{h}_{\mathrm{f}}\right)$ was held constant for all designs at $2.8 \pm 0.2 \mathrm{~mm}$.

Table 2: Design parameters of the E-glass fiber corrugated core along with the measured panel core density and relative density of the test structures with measured and predicted values for the open corrugated core.

\begin{tabular}{|c|c|c|c|c|c|c|c|c|c|}
\hline & \multirow{2}{*}{$\begin{array}{c}\mathrm{t} \\
(\mathrm{mm})\end{array}$} & \multirow{2}{*}{$\begin{array}{c}1 \\
(\mathrm{~mm})\end{array}$} & \multirow{2}{*}{$\begin{array}{c}\mathrm{H} \\
(\mathrm{mm})\end{array}$} & \multirow{2}{*}{$\begin{array}{c}\mathrm{c} \\
(\mathrm{mm})\end{array}$} & \multirow{2}{*}{$\begin{array}{c}\omega \\
\text { (degree) }\end{array}$} & \multicolumn{2}{|c|}{$\begin{array}{l}\text { Core Density } \\
\left(\mathrm{kg} / \mathrm{m}^{3}\right)\end{array}$} & \multicolumn{2}{|c|}{$\begin{array}{c}\text { Open Core } \\
\text { Relative Density (\%) }\end{array}$} \\
\hline & & & & & & Open & Filled & Measured & Predicted \\
\hline Design 1 & 3.5 & 14.1 & 14.1 & 1.8 & 60.6 & 758 & 774 & 45.1 & 40.4 \\
\hline Design 2 & 1.75 & 23.8 & 21.6 & 1.0 & 59.6 & 282 & 355 & 16.8 & 15.0 \\
\hline Design 3 & 3.5 & 24.9 & 23.8 & 1.9 & 61.3 & 440 & 518 & 26.2 & 26.7 \\
\hline Design 4 & 5.25 & 24.8 & 24.3 & 3.15 & 58.4 & 580 & 650 & 34.6 & 35.8 \\
\hline
\end{tabular}




\subsection{Material Selection and Properties}

\subsubsection{E-Glass Fiber}

The E-glass fiber used in the core of the sandwich structure has a high strength, and is commonly used in transportation applications [22]. E-glass fibers are composed of silica (54.3 wt $\%$ ), alumina (15.2 wt $\%$ ), calcium oxide (17.2 wt $\%$ ), magnesium oxide (4.7 wt $\%)$, boron oxide (8.0 wt $\%)$, and sodium oxide $(0.6 \mathrm{wt} \%)$. The fiber tensile strength of commercial fibers is reported to be range from 1.7-2.5 GPa with a Young's modulus of 72-81GPa [101]. The density of E-glass fiber is $2.54 \mathrm{Mg} / \mathrm{m}^{3}$. The core web was constructed from a 3D woven fabric using Hybon 2022 silane sized, E-glass fibers with an average fiber diameter of $\sim 18 \mu \mathrm{m}$.

The Young's modulus of the E-glass fibers was obtained by testing entire warp and weft tows extracted from the 3D fabric in quasi-static tension utilizing grooved capstan grips at a strain rate of $10^{-3} \mathrm{~s}^{-1}$ following test methods in ASTM D-2343. The fiber tows were wrapped around the capstan grip, clamped into place, and pre-tensioned to approximately $10 \mathrm{MPa}$ to remove fiber slack. The stress-strain response was initially linear until the onset of isolated fiber failure within the fiber tow, Figure 28. The E-glass fiber elastic modulus was $74 \mathrm{GPa}$ and lay within the literature range of values of $72-81 \mathrm{GPa}[22]$. The measured tensile strength of the fiber tows extracted from the woven fabric was approximately $1.0 \mathrm{GPa}$, much lower than the reported virgin fiber strength immediately on formation of 3.4 GPa [22]. Mechanical processing of the fibers and silane processing is known to reduce the tensile strength by $50 \%$ dropping the predicted strength of silane coated E-glass to $1.7 \mathrm{GPa}$, Figure 28 . It is estimated that the fiber handling during the 3D weaving process further degrades the fiber strength as seen when the silane coating is applied. 
The E-glass 3D fabric was comprised of approximately 2,300 fibers in the weft fiber tows and nearly 3,500 fibers in the warp fiber tows of the as-manufactured fabric. The mass per unit area of the dry (1.49 mm thick fabric) was $1.86 \mathrm{~kg} / \mathrm{m}^{2}$ for the weave pattern used in this study.

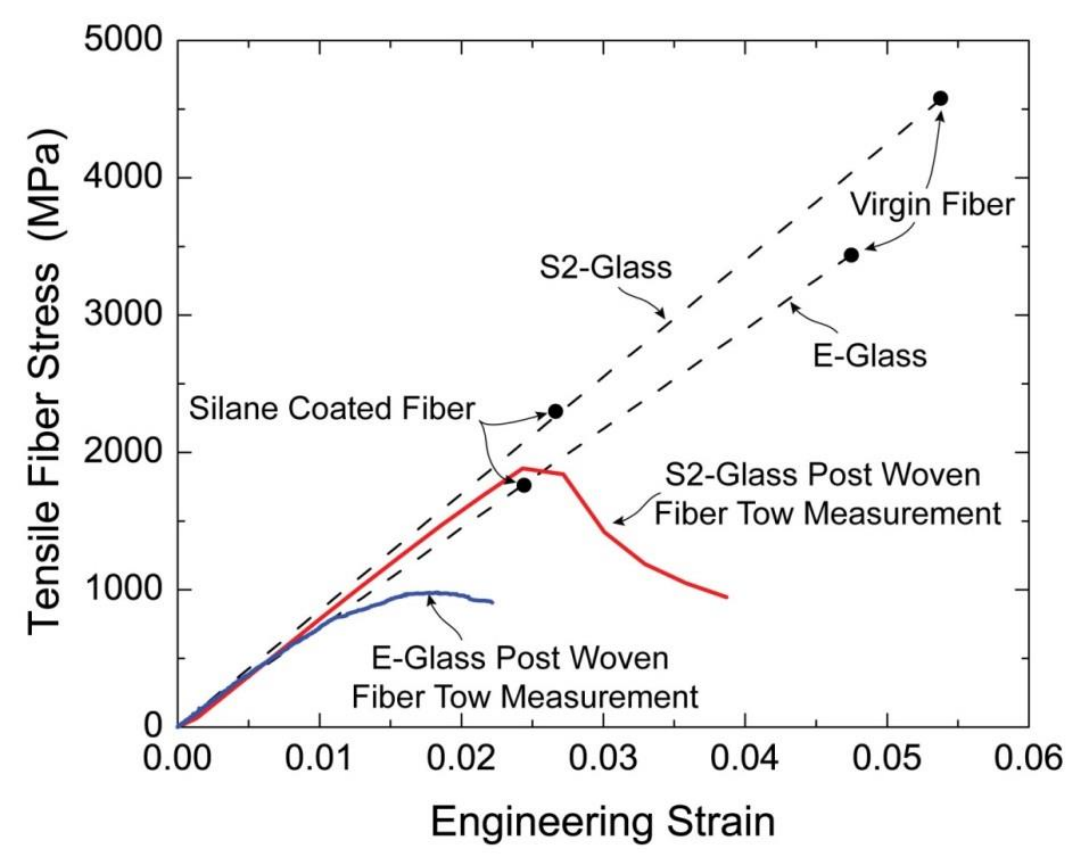

Figure 28: Measured tensile stress-strain responses of $\mathbf{E}$ and S2-glass fiber tows removed from the 3D woven fiber architectures compared to the theoretical response of ideal silane coated fibers used in this fabric.

\subsubsection{S2-Glass Fiber}

S2-glass was selected for the facesheets because of its higher tensile strength [22]. It is composed of silica (64.2 wt \%), alumina (24.8 wt \%), magnesium oxide (10.27 wt $\%)$, ferrous oxide $(0.21 \mathrm{wt} \%)$, sodium oxide $(0.27 \mathrm{wt} \%)$, barium oxide $(0.2 \mathrm{wt} \%)$, calcium oxide $(0.01 \mathrm{wt} \%)$, and boron oxide $(0.01 \mathrm{wt} \%)$. S2-glass fibers have a reported tensile strength of 2.3-3.4 GPa (in finished product form) and an elastic modulus of $86-93 \mathrm{GPa}[22,101]$. The face sheets were constructed from a S2-glass 3D woven made from AGY 463 S2-glass roving with an epoxysilane sizing. The average fiber diameter was $9 \mu \mathrm{m}$. 
The S2-glass fiber modulus was $82 \mathrm{GPa}$; slightly below the literature value of $85 \mathrm{GPa}$ [22]. The measured tensile strength of the fiber tows extracted from the woven fabric was approximately 1.9 GPa for the S2-glass fibers. The S2-glass virgin fiber strength immediately on formation is reported to be $4.5 \mathrm{GPa}$ [22]. Similar to the E-glass fiber, mechanical processing of the fiber and silane processing is known to reduce the tensile strength by $50 \%$ dropping the predicted strength of silane coated S2-glass to 2.25 GPa. Similar to the E-glass fiber, it is estimated that the $3 \mathrm{D}$ weaving process further degradates the fiber strength.

The S2-glass 3D fabric contained nearly 8,000 fiber filaments in a weft tow and 11,000 fiber filaments in a warp fiber tows. The density of S2-glass is $2.49 \mathrm{Mg} / \mathrm{m}^{3}$ and the aerial density of the $3.6 \mathrm{~mm}$ thick dry fabric was $3.39 \mathrm{~kg} / \mathrm{m}^{2}$. S2-glass was considered for use in the core but was eliminated from the study due to the tight weave process of the S2-glass 3D weave which significantly restricted the flexibility of the fabric make core manufacture with the off-the-shelf S2-glass product very difficult.

\subsubsection{Kevlar $^{\circledR}$ Fiber}

$\operatorname{Kevlar}^{\circledR} 29$ is used to bond the facesheets to the composite core to provide a high strength nodal attachment. $\operatorname{Kevlar}^{\circledR}$ is an organic fiber in the aromatic polyamide (aramid) family commonly used in safety clothing, body armor, marine and aerospace applications [102]. Kevlar $^{\circledR} 29$ is a high strength, high modulus material with a reported density of $1.44 \mathrm{~g} / \mathrm{cm}^{3}$, a breaking tenacity of 2,920 MPa, a tensile modulus of 70-80 GPa, and maximum failure strain of $3.6 \%[103]$

The Kevlar $^{\circledR}$ thread used in this structure is a Commercial Size 69 thread (Commercial size 69 also corresponds to a Tex size 70, Denier size 630, and Government Size E). A thread is 
a bundle of fiber rovings twisted together to form a tight grouping of intertwined fibers bundles.

This thread consists of three roving bundles that are braided together to allow operation in a sewing machine. The modulus of the $\operatorname{Kevlar}^{\circledR} 29$ thread was obtained by the method presented in the ASTM D-2343 test method. The Kevlar ${ }^{\circledR}$ thread fixed to grooved capstan grips and strained at a rate of $10^{-3} \mathrm{~s}^{-1}$ under tensile loading. The stress-strain response was linear until catastrophic tensile yield was observed, Figure 29. The Kevlar ${ }^{\circledR}$ threads elastic modulus was 76 GPa and assessed to be slightly lower than peak virgin values due to the material handling and associated damage during manufacture of the thread. The measured tensile tenacity was $2,830 \mathrm{GPa}$, a very close comparison with virgin values.

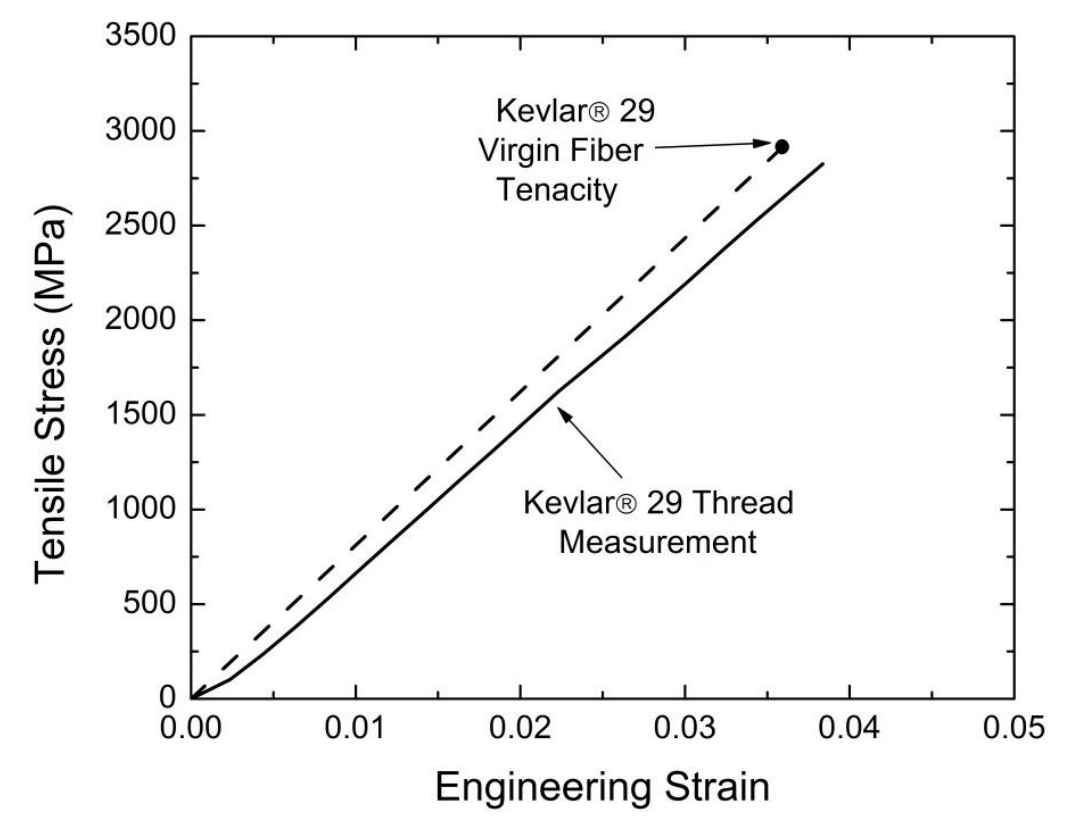

Figure 29: A representative tensile stress measurement for the Kevlar thread and compared to virgin fiber tensile tenacity at strain failure [103]. 


\subsubsection{SC-11 Epoxy}

A unique epoxy, SC-11, was chosen for use as the matrix material within this hybrid composite structure to provide structural support, shock resistance, and provide the necessary viscosity required for a vacuum infusion manufacturing process. SC-11 epoxy, manufactured by Applied Poleramic Inc. (Benicia, California), is two-part, rubber-toughened epoxy initially developed for ballistic applications for United Defense [104]. This epoxy resin provides about 90 minutes of working time and provides excellent adhesion to E and S2-glass fibers, aluminum alloys, and to itself even after full cure has occurred. SC-11 epoxy was the first epoxy product that would pass ballistic shock requirements. While definitely not the highest strength resin available on the market, the combined properties made it a good choice for this application.

The measured density of this epoxy was consistent with manufacturer specifications of $1.05 \mathrm{~g} / \mathrm{cm}^{3}$. A compressive stress-strain response of the rubber-toughened epoxy was conducted following ASTM D-695at a quasi-static strain rate of $10^{-3} \mathrm{~s}^{-1}$ and an ambient temperature of $25^{\circ} \mathrm{C}$. As shown in Figure 30, the compressive yield strength of the matrix was $47 \mathrm{MPa}$ at a yield strain of $4.5 \%$. The elastic modulus measured to be $1.35 \mathrm{GPa}$. Neither manufacture reported properties or prior published properties could be located for a comparative purpose. A shear test on the matrix was additionally performed on the matrix using an Iosipescu shear fixture following test methods in ASTM D-5379. The shear stress-strain response is superimposed on Figure 30. The shear strength, $\tau_{\mathrm{m}}$, of the matrix was $\sim 22 \mathrm{MPa}$ at a yield strain of $11.5 \%$. The shear modulus was $210 \mathrm{MPa}$. Unsuccessful attempts to obtain tensile measurements occurred due to equipment limitations. 


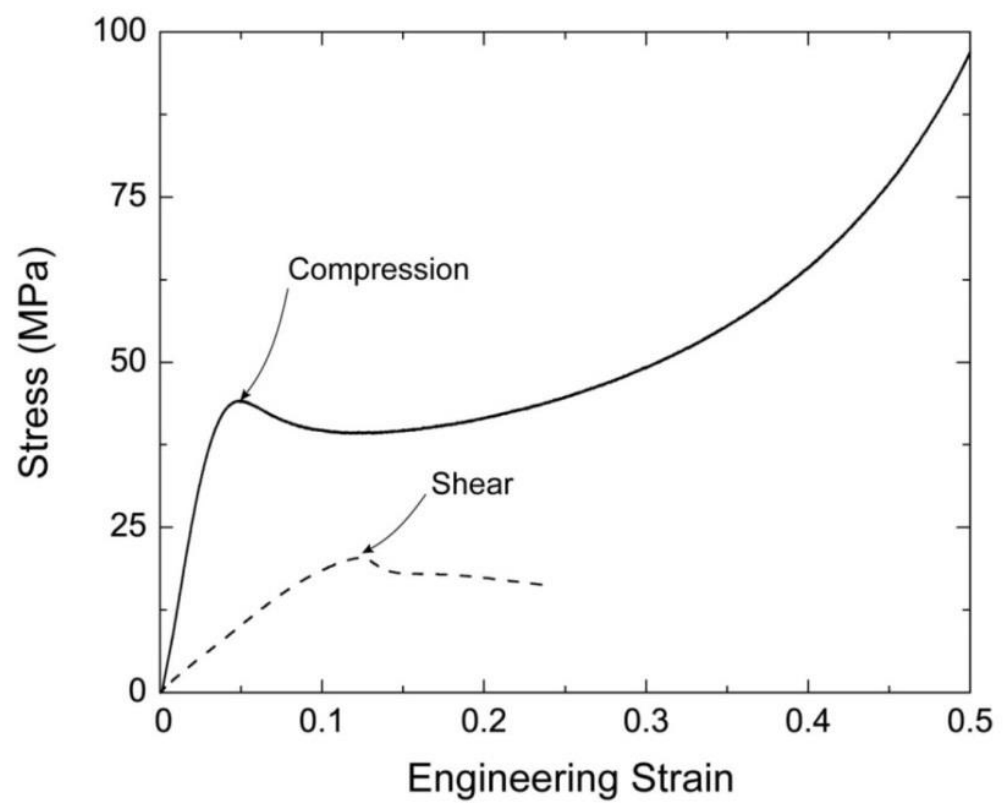

Figure 30: Compressive and shear stress-strain response for the rubber toughened SC-11 epoxy resin used as the matrix in the hybrid composite structure.

The SC-11 epoxy was further characterized through the use of a Q800 Dynamic Mechanical Analysis (DMA) test apparatus developed by TA Instruments to investigate the viscoelastic behavior and locate the glass transformation temperature of the material. Applying a sinusoidal stress at a frequency of $1 \mathrm{~Hz}$ allowed for the determination of the storage modulus, E', the loss modulus, E', the elastic/Young's modulus, E, the loss tangent, $\tan \delta$, and the glass transformation temperature. The storage modulus is a measure of the stored energy during elastic deformation and the loss modulus is a measure of the energy converted to heat during deformation. The ratio of the loss modulus to the storage modulus provide the loss tangent as seen in Equation 4.1;

$$
\tan \delta=\frac{E^{\prime \prime}}{E^{\prime}}
$$


The loss tangent is plotted in Figure 31 for the SC-11 epoxy resin along with two other resins for comparison, SC-1 and vinyl ester. These epoxies were being evaluated for use as the matrix material, but ultimately exhibited too brittle of a response under loading. The glass transformation temperature (the temperature where the properties of a material transition from a glassy phase to a rubbery phase) are determined by the localized peak of the loss tangent. The glass transformation temperature of SC-11 was determined to be $40^{\circ} \mathrm{C}$, a viable (albeit slightly less than ideal) temperature limit to allow the study to proceed.

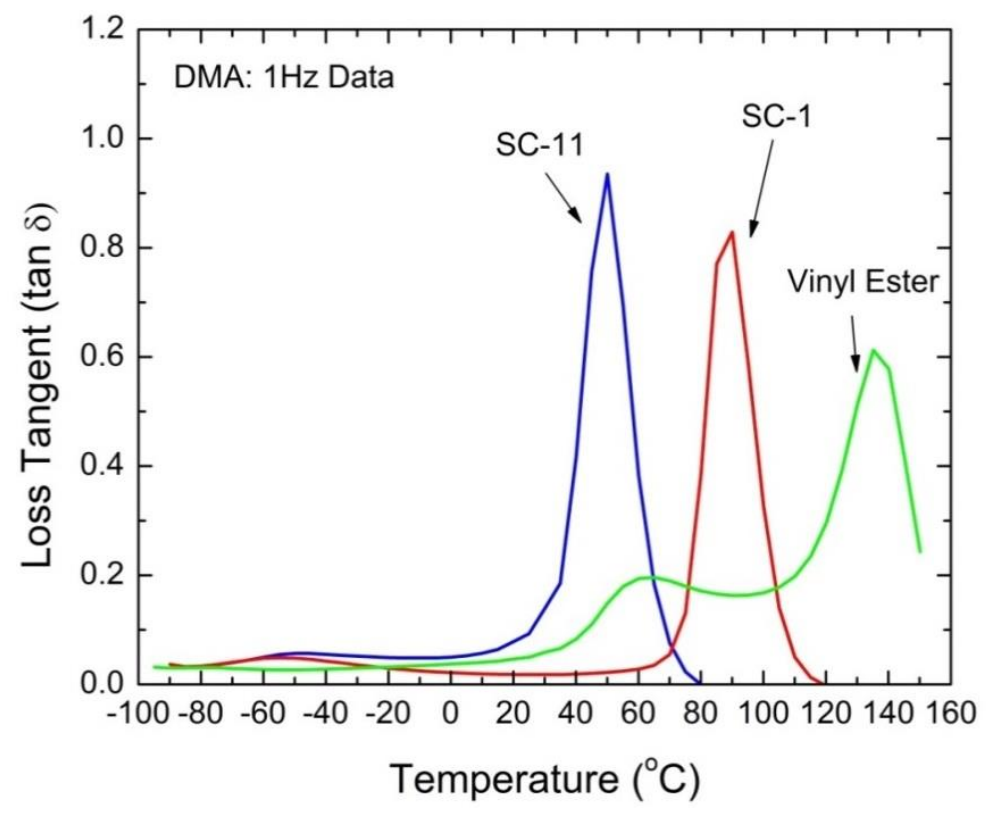

Figure 31: The loss tangent $(\tan \delta)$ is plotted as a function of temperature to reveal the glass transformation temperature of the SC-11 epoxy resin. SC-1 and vinyl ester are shown for comparison.

Finally, the elastic/Young's modulus of the epoxy can be determined as a function of temperature. The elastic modulus, plotted in Figure 32 is determined through Equation 4.2;

$$
E=\sqrt{E^{\prime 2}+E^{\prime \prime 2}}
$$

The result shows that SC-11 has a moderately constant modulus from 3-4 GPa below a temperature of $5^{\circ} \mathrm{C}$. However, the modulus begins to show a greater dependence on temperature 
between $5^{\circ} \mathrm{C}$ and $40^{\circ} \mathrm{C}$ (glass transformation temperature) varying from $2 \mathrm{GPA}$ to $0.5 \mathrm{GPA}$ in this region. SC-1 and vinyl ester resins exhibit greater consistency in the elastic modulus all the way to the $40^{\circ} \mathrm{C}$ temperature limit.

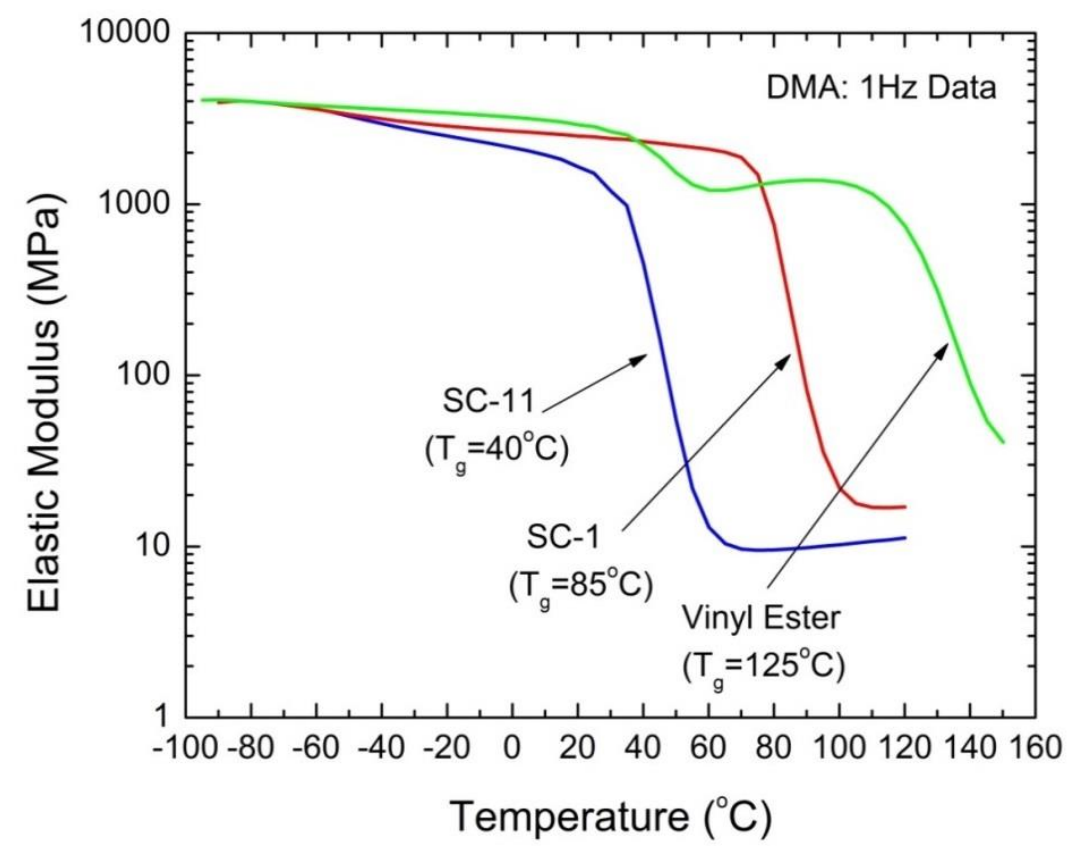

Figure 32: The elastic modulus of SC-11 epoxy is plotted as a function of temperature. SC1 and vinyl ester resins are plotted for comparison.

\subsubsection{Divinycell H130 Foam}

Similar to the foam sandwich presented by Latourte, Divinycell foam was used in the core of the hybrid structure. Divinycell foam is a closed cell rigid polyvinyl chloride (PVC) foam, manufactured by DIAB Group, that will allow the necessary support of the core during fabrication and infusion without letting the epoxy resin permeate into the foam core, thereby artificially increasing the density of the foam. Divinycell H130 (part of the DIAB H-series foams) was chosen because it has been well characterized as an energy absorbing foam and is easily obtained. The density of H130 Divinycell foam is $130 \mathrm{~kg} / \mathrm{m}^{3}$ and exhibits a relative density of $7 \%$ when compared with bulk PVC $\left(\rho=1860 \mathrm{~kg} / \mathrm{m}^{3}\right)$. The compressive stress-strain 
response of the Divinycell H130 foam was measured under quasi-static compression at a strain rate of $10^{-3} \mathrm{~s}^{-1}$ and the stress-strain response is shown in Figure 33. The yield strength of the foam was $\sim 3.0 \mathrm{MPa}$ at a yield strain was $\sim 3.5 \%$. The elastic modulus was measured to be $\sim 100$ MPa. These values are all in agreement with manufacturer reported values [105]. We note that the Divinycell foam exhibits a Calladine and English [106] type I (plateau) stress-strain response and has been found to exhibit a strength that is less sensitive with loading rate [106].

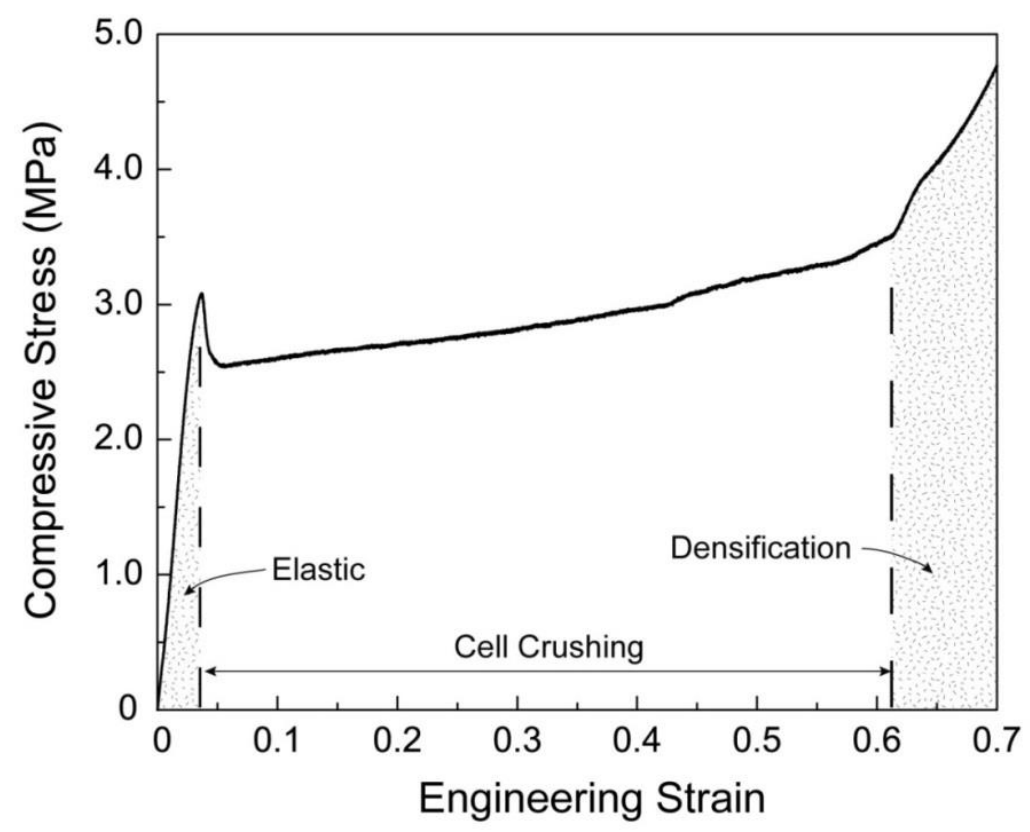

Figure 33: Compressive stress-strain response of H130 Divinycell foam.

\subsection{D Woven Fiber Architecture}

Composite delamination is one of the primary failure mechanisms during in-plane compressive. The delamination strength is affected by the matrix interface strength between plies and without the addition of stronger material to bear a portion of the load, the matrix often defines the critical strength of a composite laminate. The implementation of an out-of-plane, 
binding fiber strategy through concepts such as z-pinning, through stitching, or three dimensional (3D) weaving reduces susceptibility to delamination failure during both quasi-static and dynamic loading [107]. Experimental studies have shown that delamination cracks are partially or (in some cases) entirely eliminated by 3D weaving, and in some cases flexural strengths can double those of 2D laminates [107].

A solution to reducing delamination is to add through thickness fibers that will provide thickness deformation resistance. In a 3D weave, the out-of-plane fiber tows (z-yarns), are woven perpendicular to all other fiber tows, binding them together. Under in-plane compression of the composite, the out-of-plane expansion of the warp and weft reinforced laminates is inhibited by the z-yarn which is then placed in tension. Non-crimp 3D orthogonal fiber architectures are produced by 3Tex, Inc. (Cary, NC), for the specific purpose of creating a delamination resistant composite. 3Tex offers the capability to weave glass, carbon, aramids, and other advanced fibers, in a variety of fiber distributions for optimization under a specific application.

Standard available balanced fabric 3Tex products were chosen for the research to reduce the associated cost of the products. A 54oz E-glass fiber $3 \mathrm{Weave}{ }^{\circledR}$ woven mat (PN: P3WGE045) was chosen for the core fabric and a $100 \mathrm{oz}$. S2-glass fiber 3 Weave ${ }^{\circledR}$ woven mat (PN: P3W-GS025) was chosen for the facesheets. The fiber architecture of both 3D woven textiles consists of alternating layers of originally straight warp and weft fiber tows ${ }^{3}$ held in place by a smaller fraction of z-yarns that loop over and under the weft tows parallel to the warp tow direction. The $3 \mathrm{D}$ fabric used here consists of a $\left[0^{\circ} / 90^{\circ} / 0^{\circ} / 90^{\circ} / 0^{\circ}\right]$ lamination of 3 weft and 2

\footnotetext{
${ }^{3}$ Weft tows are also referred as fillers, warp tows as stuffers, and z-yarn tows as warp weavers.
} 
warp layers with an integrated z-yarn. The z-yarn extends to the outermost weft tows, binding the entire structure and thereby increasing the delamination strength [108]. The 3D fiber architecture is illustrated in Figure 34.

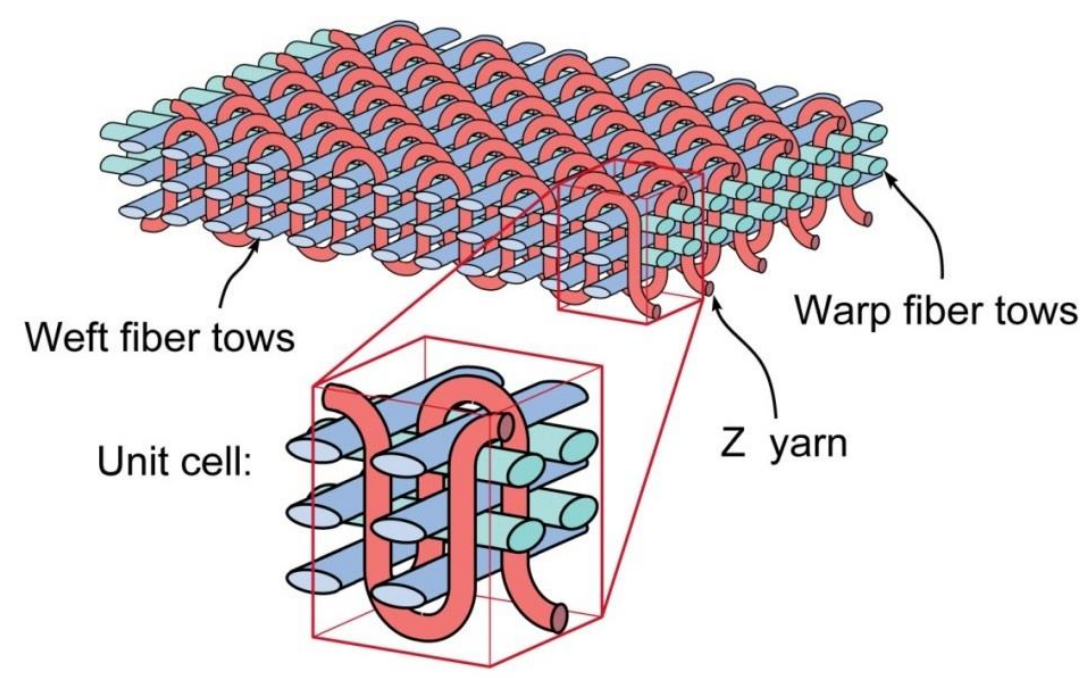

Figure 34: Fiber architecture of the 3D woven glass fiber fabrics used in the core struts and face sheets of the corrugated core sandwich structure. The 3D woven geometry consists of three weft tows, two warp tows and one z-yarn per repeating volume element.

Ideally, a 3D woven fabric will have a z-yarn exhibits square-wave profile that minimally deflects the warp and weft fiber tows [107] while maintaining binding confinement of these tows. In practice, a quasi-sinusoidal profile is usually created because of material flexibility limitations and manufacturing limitations that cause tow displacement as tension is applied during the z-yarn insertion process. The result is an inevitable degree of warp and weft tow fiber waviness. While many studies confirm a significant delamination resistance benefit of 3D woven textiles, the fiber waviness created within the in-plane warp and weft fiber tows increases susceptibility to kink band formation and microbuckling failure under in-plane compressive loading $[107,109,110]$. Efforts are made to minimize the tow waviness for an optimal product. 
The number of warp and weft layers, fraction distribution between direction, number and type of fibers per tow, and the spacing between tows in each layer are variables that the effect structure and mechanical properties of the design. The fiber fraction and number of tows in each direction for both the E and S2-glass woven fabrics is summarized in Table 3. Approximately $48 \%$ of the fibers were in the warp tows, $48 \%$ in the weft tows with the remaining $4 \%$ residing in the z-yarn.

Table 3: 3 Weave ${ }^{\circledR}$ fiber distribution in the warp, weft, and $\mathrm{z}$ tows for both E-glass and S2glass.

\begin{tabular}{|c|c|c|}
\hline Material & $\begin{array}{c}\text { Fiber Fraction (\%) } \\
\text { Warp / Weft / Z-Yarn }\end{array}$ & $\begin{array}{c}\text { Number of Tow Layers } \\
\text { Warp / Weft }\end{array}$ \\
\hline E-Glass & $48.3 / 47.9 / 3.8$ & $2 / 3$ \\
S2-Glass & $45.9 / 49.6 / 4.5$ & $2 / 3$ \\
\hline
\end{tabular}

There are differences between the fabrics used, most notable and difficult to characterize, is the fabric flexibility. Fabric flexibility is affected by the weave's z-yarn spacing, z-yarn fiber tension, and the tow spacing. The E-glass $3 \mathrm{Weave}{ }^{\circledR}$ is a looser weave which allows the fabric to be easily folded, in contrast to the tight S2-glass $3 \mathrm{Weave}{ }^{\circledR}$. The z-yarn spacing in the E-glass weft and warp directions was approximately $5.2 \mathrm{~mm}$, Figure 35(a). This fabric successfully provided the flexibility needed to allow folding in the weft tow direction to create the corrugated core sandwich panels $[37,73]$. The $\mathrm{S} 2$-glass $3 \mathrm{Weave}{ }^{\circledR}$ is much more rigid in the dry fabric form due to the manufacture. The measured inter z-yarn spacing in S-2 glass warp direction was approximately $5.0 \mathrm{~mm}$, Figure 35(b). The higher weave density than the E-glass limited the use of this specific S2-glass product for consideration as the core webs. 


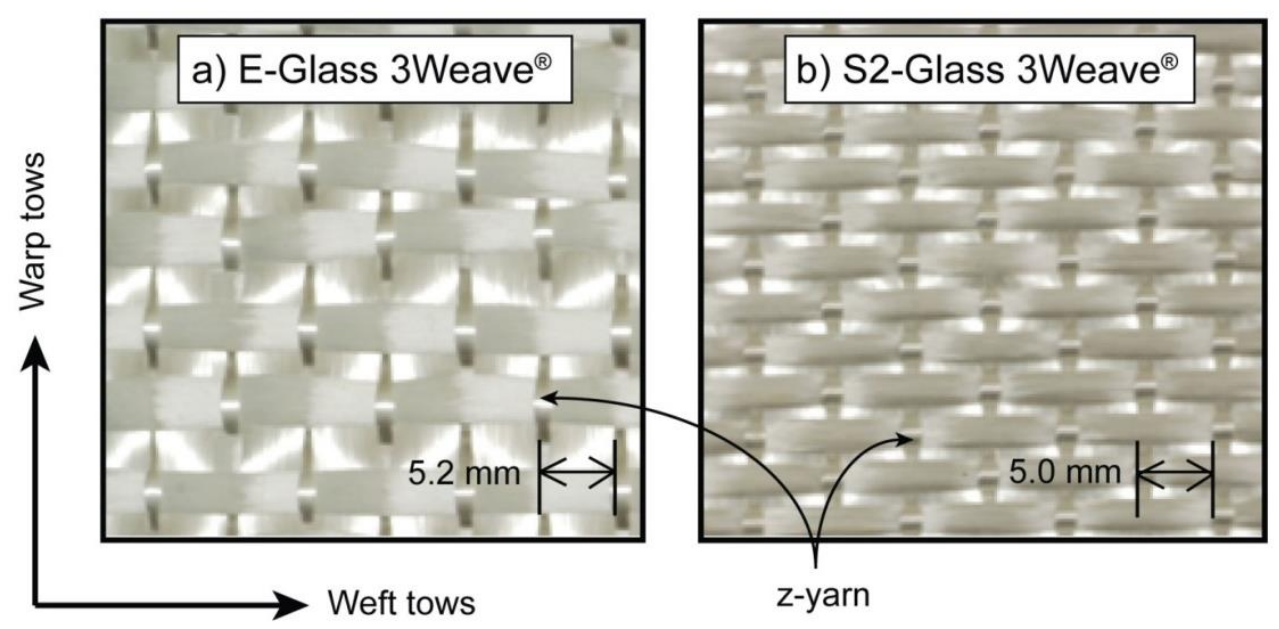

Figure 35: Photograph of the (a) E-glass 3D woven fabric (3Tex 3 Weave ${ }^{\circledR}$ fabric) used for the core struts and (b) the S2-glass $3 W e a v e{ }^{\circledR}$ used for the face sheets. The E-glass $3 W$ eave ${ }^{\circledR}$ fabric had a larger $z$-yarn spacing resulting in a looser weave which facilitated fabric folding to form a core corrugation.

\subsection{Relative Density}

The relative density is a useful design metric in the study of the associated failure mechanisms of a structure. Specific to a corrugation, the slenderness ratio (t/l) if often modified to create structures with varying relative densities to investigate the limits of geometric driven failure (buckling) and material properties driven failure (yielding). The hybrid corrugated core sandwich panel possesses a slightly different geometry, as compared to an ideal corrugated, because the core webs are folded over the nodal apexes, Figure 27. The increase in a slenderness ratio (t/l) also increases the nodal separation distance, $\mathrm{c}$, also shown in Figure 27. The result is a slight variation in the overall hybrid-corrugated core sandwich structure when changing the slenderness ratio. This difference is hypothesized to be negligible in the structure performance and the relative density is calculated to account for this discrepancy. 
The relative density, $\bar{\rho}$, of the open core structure is determined by the volume occupied by the corrugated core in a unit cell of the core, Equation 4.3, divided by the unit cell volume, Equation 4.4 and is given by Equation 4.5.

$$
V_{\text {UnitCell }}=\frac{2 H}{\sin \omega}(t-2 c \cos \omega+H \cos \omega)
$$

$$
V_{\text {Core }}=\frac{2}{\sin \omega}\left(t H-c^{2} \cos \omega\right)
$$

$$
\bar{\rho}=\frac{V_{\text {core }}}{V_{\text {UnitCell }}}=\frac{t H-c^{2} \cos \omega}{H(t+H \cos \omega-2 c \cos \omega)}
$$

When $t H>>c^{2} \cos \omega$, Equation 4.5 can be simplified to give to relative density of the open core;

$$
\bar{\rho}=\frac{t}{t+H \cos \omega-2 c \cos \omega}
$$

The measured and predicted open-core relative densities of the four core designs are given in Table 2. The measured values are slightly higher than predications due to the accumulation of resin at the foam-web interface (resulting from gaps between the foam prisms created during the panel assembly process). 


\section{Chapter 5}

\section{Fabrication Methodology}

The hybrid foam glass-epoxy corrugated cellular structure sandwich panel design is unique without any prior design or manufacture of this structure before this study. This equally means there were no known manufacturing processes to base the manufacturing process. Manufacturing this structure entirely by a hand lay-up method might suffice for several prototypes, but not for the needed structure repeatability that is desired. Further, using a RTM process was too expensive to construct without perfecting a design for mass manufacture. As a result a bagged infusion method was chosen to provide the necessary infusion consistency between parts and provide the flexibility needed to tailor the manufacture process along the way to accommodate flat composite sheets, single tier corrugations, multi-tier corrugations, and larger panels up to 36 inches x 36 inches $(91.4 \mathrm{~cm}$ x $91.4 \mathrm{~cm})$. It was also determined that the desired infusion method of the entire cellular structure in a single infusion would be the correct method of manufacture. This method provides the ability to join the facesheets through a stitching process while the core is still in fabric form. If infusion occurred prior to facesheet-core joining, either the nodal attachments would solely be reliant on the matrix adhesion, or a rivet/bolt connection method would need to be employed. Further, a single step infusion of a cellular structure sandwich panel is a unique method of manufacture that has never been published or 
knowingly attempted. The manufacture of the hybrid corrugated sandwich structure is divided into four main stages:

Stage 1 - Construction of the dry fabric structure

Stage 2 - Resin infusion process

Stage 3 - Temperature and pressure cure process

Stage 4 - Post-cure pane fabrication

The discussion of the manufacturing processes is similar for the laminated composite sheets, single tier structures, and multi-tier structures investigated in this research study. Variations in the process for each structure are discussed as each stage of the overall process is presented.

\subsection{Dry Fabric Hybrid Structure Manufacture}

The process of assembling the hybrid corrugated sandwich structure begins with the manufacture of the components. Divinycell foam is cut into long, triangular prismatic bars. These bars are cut into equilateral triangles with a triangular height of $25.4 \mathrm{~mm}$ or $14.5 \mathrm{~mm}$. Additionally, the 3D woven fabric is cut to length and temporarily clamped together before beginning the sewing process. The sewing process is illustrated in Figure 36. The process of stitching the S2-glass facesheets to the E-glass core is a labor intensive process requiring the continual folding and bending of the structure. Depending on the desired slenderness ratio of the core, a single, double or triple laminate of the E-glass 3D woven fabric was sewn into the structure. Using a thinner E-glass fabric was needed to obtain the necessary flexibility to forming the core. Laminating the core maintained core flexibility as each laminate could slide relative to its neighbor while still in a partially unstitched, dry fabric form. 
Machine stitching requires access to both the outer edges of fabrics being joined. As a result, the entire corrugation cannot be created with the facesheets being joined as a final step. Instead, the stitching is entirely completed one unit cell at a time. Stitching begins by aligning the warp tows parallel with each other and stitching the edges of the fabric together parallel to a warp tow. Kevlar® 29 thread is stitched using a locking straight stitch (also known as a lock stitch), Figure 37, set at approximately 6 stitches per $\mathrm{cm}$ to join the corrugated core and the fabric facesheets. For increased strength at the attachment points, a second full length stitch is added coincident with the first stitch. As each full length stitch is completed, a foam prism is placed adjacent to the newly formed core web and the foam prism is stitched into place. Because stitching of both facesheets occurs simultaneously during the sewing process, the structure must be completely removed from the machine and flipped over to provide access to the necessary stitch location for each nodal apex. Since triangular corrugations only provide a small area at the nodal apex for nodal attachment, the straight stitch was the only viable choice for stitching. The process presented is then repeated the necessary amount of times to create the desired structure shape. An Artisan Toro 4500 Heavy Duty sewing machine was used for the process, which limited structure width to 16 inches $(406.4 \mathrm{~mm})$.

Single, double and triple laminates of E-glass 3D woven fabric was used to create both flat laminated sheets and the hybrid laminated 3D woven cores. Laminating 1, 2 or 3 layers of the E-glass fabric resulted in struts with dry thicknesses of 1.49, 2.98, and $4.47 \mathrm{~mm}$. Multi-tier corrugated sandwich structures were created by stacking single tier structures while in the dry fabric form. 

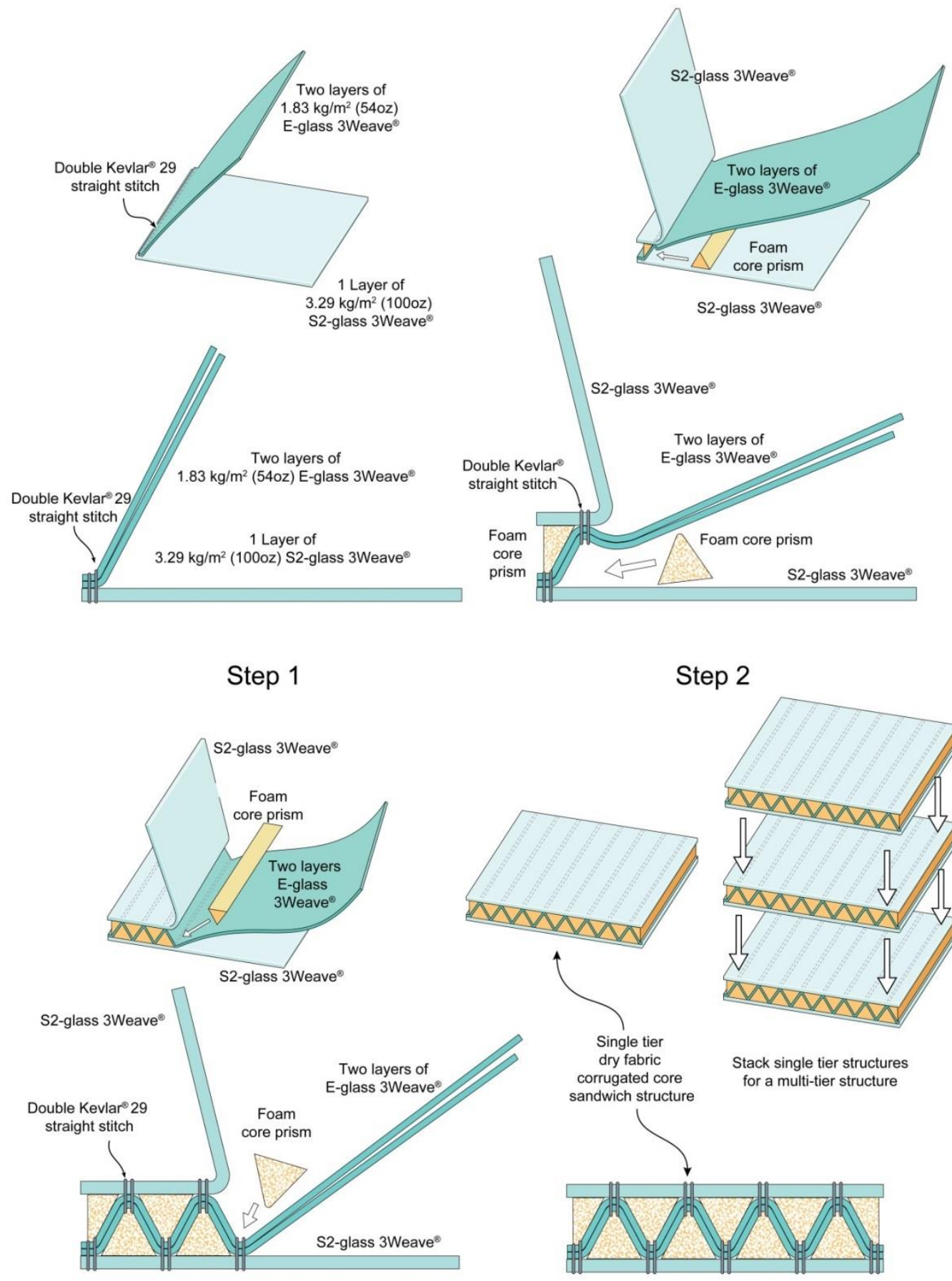

Step 3
Step 2

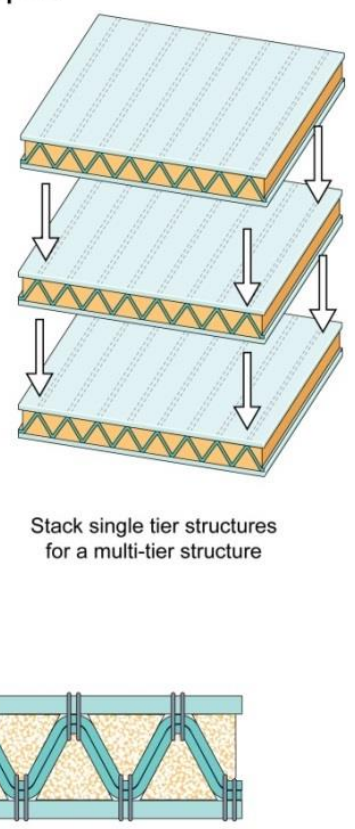

Step 4

Figure 36: Schematic illustration of the assembly process of the hybrid corrugated sandwich panel. 


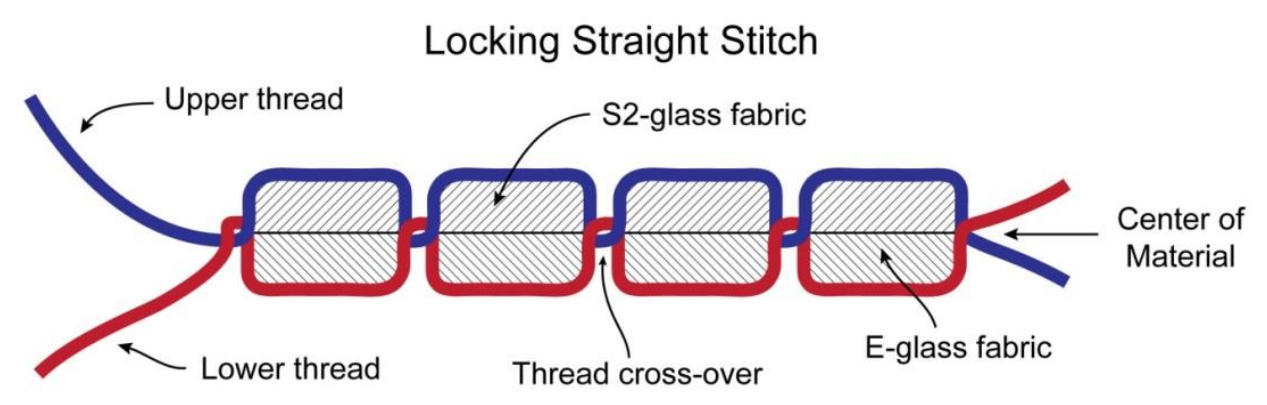

Figure 37: A locking straight stitch, commonly called a lockstitch, was used for nodal coreto-facesheet connections.

\subsection{Vacuum Infusion Process}

Every composite panel or structure was manufactured using a Vacuum Infusion Process (VIP) to provide trapped air pockets, optimize resin infiltration, and provide the ability to alter fiber volume fractions. As illustrated in Figure 38, the VIP uses a vacuum pump to create a pressure differential within a vacuum bag formed mold. The bagged mold is attached to a resin supply, so that when a pressure differential is established, the atmospheric pressure outside of the bagged part pushes the resin into the mold. The advantage of a VIP is not only to pull the resin through the part, but to remove any air pockets that may form voids. A resin trap is placed between the mold and vacuum pump to catch any excess resin that flows through the part when full infiltration occurs. The VIP process helps reduce resin waste (through reduced amounts of resin needed for manufacture) and contain resin vapors for a safer work environment. 


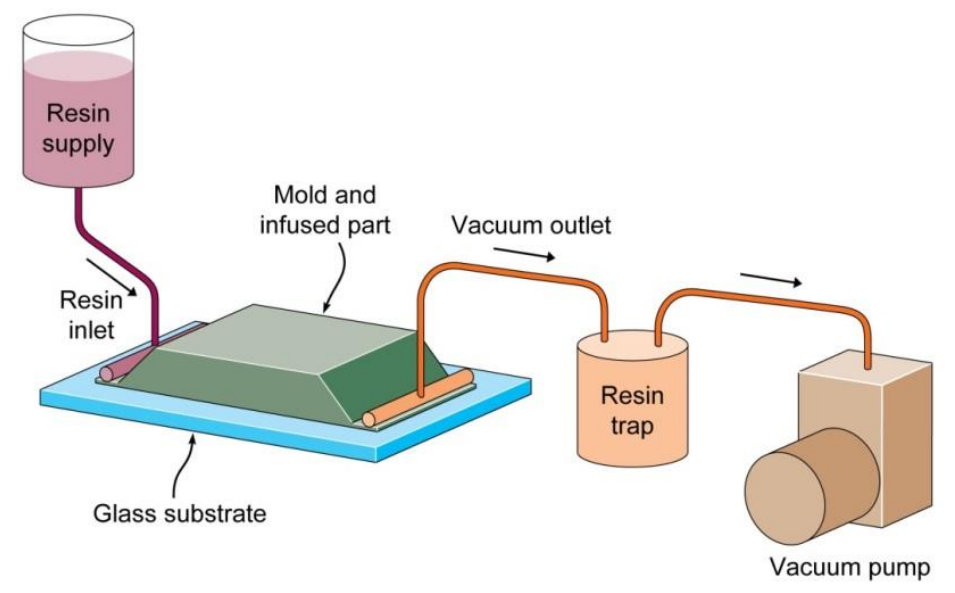

Figure 38: Basic layout of the Vacuum Infusion Process (VIP) used for resin infiltration of the composite structure.

The specific process of resin infusion being used is often referred to as Vacuum Assisted Resin Transfer Molding (VARTM). However, this method does not entirely use rigid molds to form the part cavity for the composite. Rather, the composite is built on a rigid glass substrate and a vacuum environment is created through the use of an air-tight, high-temperature nylon bag sealed to the substrate with a vacuum sealant tape to prevent any leaks from forming. This technique is both similar to RTM and bag molding techniques and is illustrated in Figure 39.

Aside from the ballistic shock resistant properties, SC-11 epoxy was chosen for use as the matrix because it was designed use with VARTM processes. After mixing, the epoxy system had a viscosity of $900 \mathrm{cps}$, sufficient to permit vacuum assisted infiltration of a $500 \mathrm{~mm} \times 500 \mathrm{~mm}$ corrugated glass core panel in 30 minutes. 


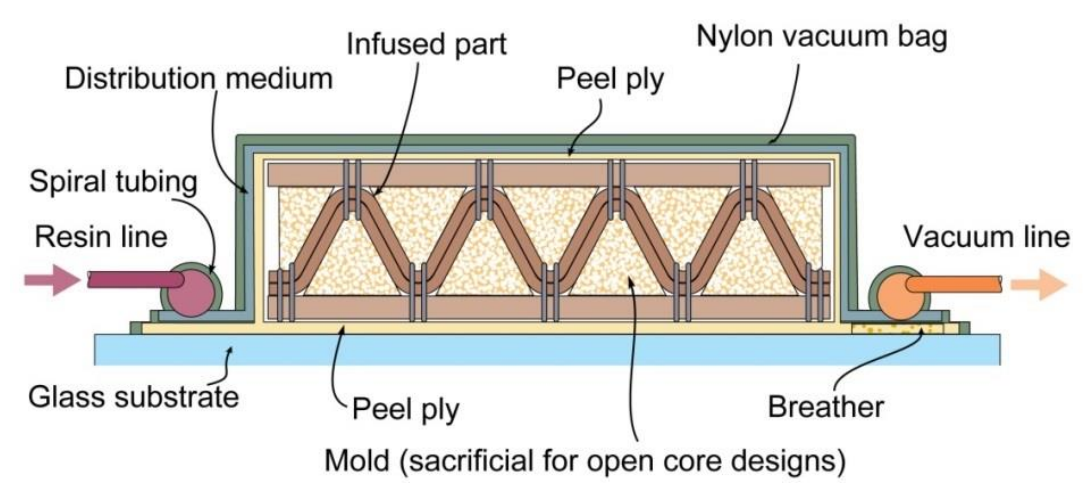

Figure 39: A slightly modified Vacuum Assisted Resin Transfer Mold (VARTM) technique uses a vacuum bag sealed to a rigid glass substrate to create a vacuum cavity for the part infusion.

The lay-up of the infusion materials is critical to a successful part infusion. The dry fabric panels or structures are initially wrapped in a layer of nylon peel ply which allows for part detachment after infusion. The epoxy used will bond to most parts, but does not bond with nylon. Outside of the peel ply is a layer of breather material was wrapped around the entire part which allows vacuum pressure to reach all areas of the part prior to best achieve complete part vacuum. The wrapped system was placed on a sheet of rigid glass substrate which acts as a working surface and support base. A distribution media is placed on the top of the part and connects to the resin line inlet. The distribution media allows for a quicker flow of the resin by increasing the porosity of the flow material. For the composite panels and single tier structures, a vacuum line is seated on the far end of the part connecting to the breather material. The resin inlet line is attached to the breather material at the opposite end of the part. The entire part is sealed underneath a high-temperature nylon film that is sealed to the glass substrate to create a vacuum environment. The system is leak tested prior to infusion to assure no significant vacuum leaks exist. Once sufficient vacuum pressure has been reached the resin line is opened 
to the premixed epoxy system. Time flow is critical as the epoxy has a working life of 3.75 hours to reach a viscosity of 3000 cps.

Incompressible fluid through a porous media is described by Darcy's Law, Equation 5.1, and commonly used in the design of vacuum infusion of composites;

$$
v=\frac{k}{\psi \cdot \mu} \cdot \frac{d P}{d x}
$$

where $\mu$ is the fluid viscosity, $\psi$ the porosity, $\mathrm{v}$ the interstitial velocity, $\mathrm{k}$ the preform permeability and $\mathrm{dP} / \mathrm{dx}$ the pressure gradient. Integration of Equation 5.1 provides the expression for resin time travel. The time it takes for the leading flow front to traverse the system is a function of the square of the flow front distance, Equation 5.2;

$$
t=\frac{\mu \cdot \psi}{2 \cdot k} \cdot \frac{x^{2}}{\Delta P}
$$

Since this infusion method requires complete resin from one end of the part to the other, both the type and amount of distribution media is adjusted to locally lower the porosity on the outside edge of the part to move the resin quickly to the entire part edges. The resin infusion process uses a vacuum pressure of $3.4 \mathrm{kPa}$, which creates a pressure differential of $97.9 \mathrm{kPa}$ at atmospheric pressure. Depending on the porosity used for the specific structure, infusion can take 30-60 minutes for a $400 \mathrm{~mm}$ long structure.

Flat composite panels were constructed using the same vacuum infusion process to create replicas of the corrugated core webs and facesheets to be used for mechanical testing. The flat sheets were designed and manufactured to reproduce the thickness and fiber fractions of the corrugated sandwich structures. For these panels, construction was the same as outlined above 
with the exception that breather material was only needed on one side of the panel to provide the necessary vacuum distribution.

The multi-tier hybrid corrugated sandwich structures were also created using the same vacuum infusion process with a modification to the resin flow path. As illustrated in Figure 40, the resin inlet line was connected to the top of the part and resin was quickly distributed through the use of a spiral tubing inlet line used in conjunction with distribution media. The height of the multi-tier structures created a scenario where full resin infusion of the entire part was difficult due to the effects of gravity. Infusion from the top of the part allowed the forces both from vacuum pressure and gravity to work to the advantage of part infusion. Additionally, the vacuum line was placed in a $360^{\circ}$ circumference around the part. This allowed vacuum pressure to be distributed to all areas of base of the part. The infusion method of the multi-tier structure was more advantageous for rapid, thorough, and void-free matrix infusion.

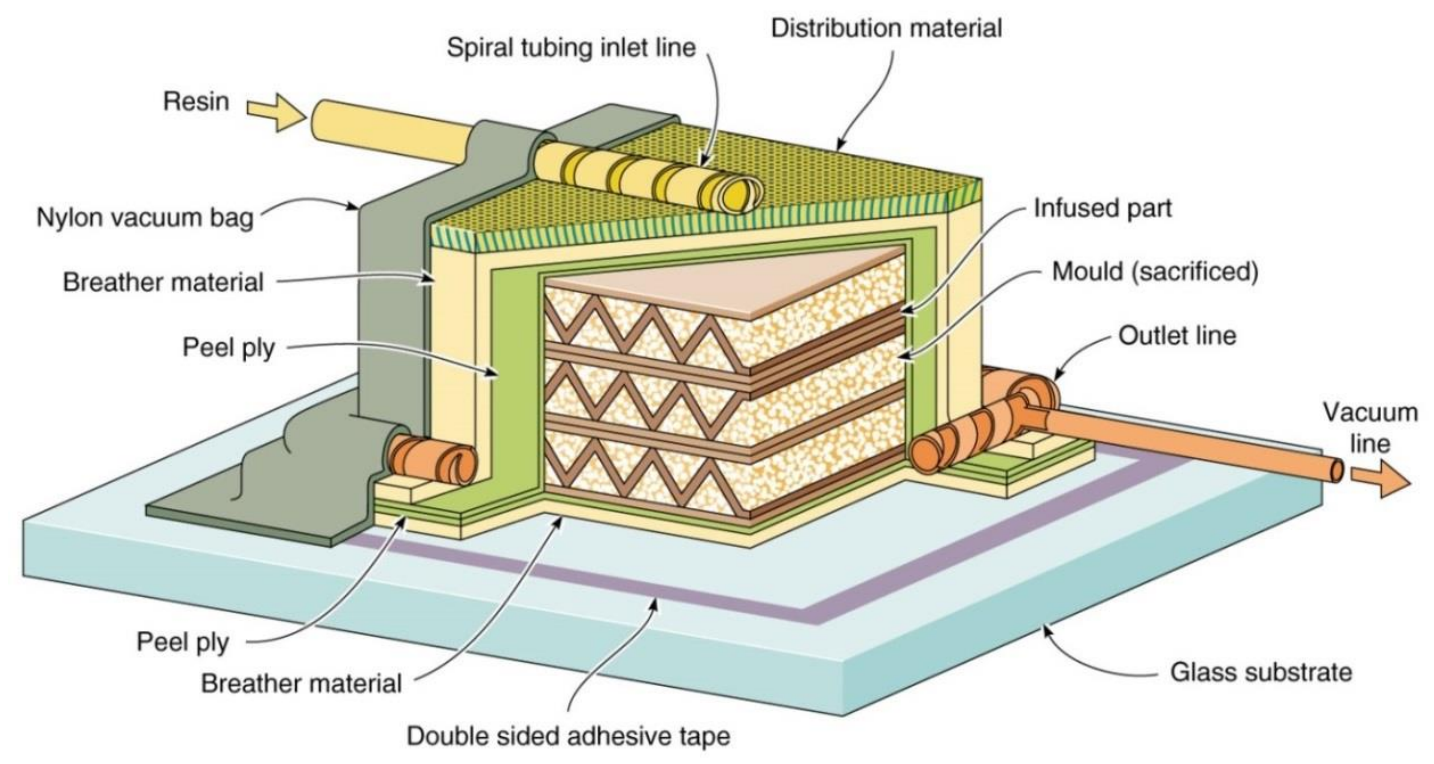

Figure 40: The vacuum infusion process of the multi-tier structure utilized a resin inlet line positioned at the top of the part and a vacuum line positioned $360^{\circ}$ around the base of the part. 


\subsection{Resin Cure Process}

The resin infusion process occurs inside of an autoclave at atmospheric pressure and ambient temperature. The ASC Process System's Econoclave EC3X5 composite autoclave, Figure 41, is used to cure every composite panel and structure created in this study. The modified-VARTM process used in combination with the autoclave facilitated removal of air voids and resin vapor within the SC-11 epoxy. During the curing process, the combination between the exothermic reaction and elevated cure temperatures has the negative effect of boiling the resin during cure thereby creating unwanted vapor pockets within the composite. Boiling of the resin occurs at much lower temperatures while under vacuum. Therefore a pressure differential technique was developed to remove the effect of resin boiling during cure.

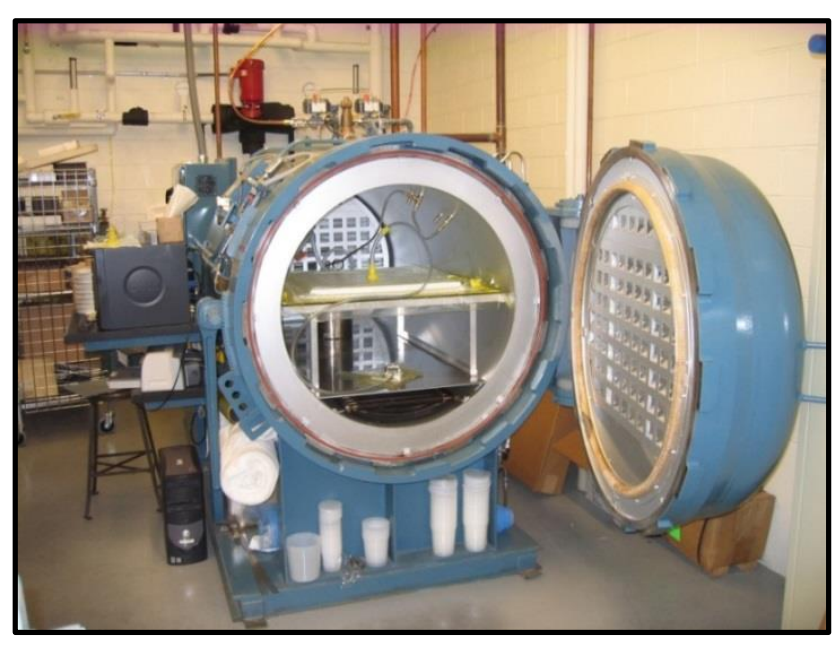

Figure 41: Econoclave EC3X5 composite autoclave was used to provide the necessary temperature and pressure for resin cure. The modified VARTM process occurs inside the autoclave at ambient temperature and pressure, as shown.

The pressure differential cure process is a unique process developed in this research study and is a new manufacture process of composite materials. This process provides the ability to control the fiber fraction of a composite and while minimizing both air and vapor voids during cure. Resin vapor is created through boiling of the resin due to low vacuum pressures and the increased temperatures from the exothermic curing reaction and elevated temperatures designed 
for cure. As summarized in Table 4 and illustrated in Figure 42, the process begins with the resin infiltration of the part at atmospheric pressure and ambient temperature. After infusion is complete, the autoclave pressure is increased to $202.6 \mathrm{kPa}$ (2 atm) to provide increased densification and fiber volume fractions (note: the pressures can be altered to provide varying fiber volume fractions). Next, a pressure difference translation occurs. The chamber pressure within the autoclave is increase at the identical rate the vacuum pressure is decreased. The resulting step maintains the pressure difference between the bagged part and chamber pressure, but allows the part pressure to increase thereby reducing the capability for the epoxy resin to boil while curing. Autoclave chamber pressures can be increased to $303.9 \mathrm{kPa}$ (3 atm) to allow the bagged part to return to atmospheric pressure $101.3 \mathrm{kPa}$ (1 atm). Finally the system is heated to $72^{\circ} \mathrm{C}$ and maintained for 4 hours for cure. After 4 hours, the temperature is slowly returned to atmospheric temperature for the removal of the part.

Table 4: Infusion and pressure differential transition cure cycle

\begin{tabular}{|l|c|c|c|c|}
\hline \multicolumn{1}{|c|}{ Resin Transfer / Cure Cycle } & $\begin{array}{c}\text { Autoclave } \\
\text { Pressure } \\
\mathrm{kPa}(\mathrm{psi})\end{array}$ & $\begin{array}{c}\text { Autoclave } \\
\text { Temp } \\
{ }^{\circ} \mathrm{C}\left({ }^{\circ} \mathrm{F}\right)\end{array}$ & $\begin{array}{c}\text { Vacuum } \\
\text { Pressure } \\
\mathrm{kPa}(\mathrm{psi})\end{array}$ & $\begin{array}{c}\text { Time } \\
\text { min }\end{array}$ \\
\hline Step 1: Prepare/bag part & $101.3(14.7)$ & $20(68)$ & $101.3(14.7)$ & -- \\
Step 2: Pull vacuum on part & $101.3(14.7)$ & $20(68)$ & $3.4(0.5)$ & 5 \\
Step 3: Infusion & $101.3(14.7)$ & $20(68)$ & $3.4(0.5)$ & $30-60$ \\
Step 4: Pressurize autoclave & $202.6(29.4)$ & $20(68)$ & $3.4(0.5)$ & 15 \\
Step 5: Vacuum release* & $202.6(29.4)$ & $20(68)$ & $101.3(14.7)$ & 20 \\
Step 6: Pressurize autoclave* & $303.9(44.1)$ & $20(68)$ & $101.3(14.7)$ & 20 \\
Step 7: Temperature cure & $303.9(44.1)$ & $72(162)$ & $101.3(14.7)$ & 240 \\
Step 8: Return to ambient & $101.3(14.7)$ & $20(68)$ & $101.3(14.7)$ & 60 \\
\hline * Process steps occur simultaneously & & \\
\hline
\end{tabular}


If not performed correctly, this process can dramatically fail, letting in large amounts of air and ruining the structural integrity of the entire composite piece. Careful attention must be given to the pressure transition rates and synchronization between the vacuum pressure within the bagged mold and the autoclave pressures. This process can fail in two ways: bag leakage and improper pressure transition.

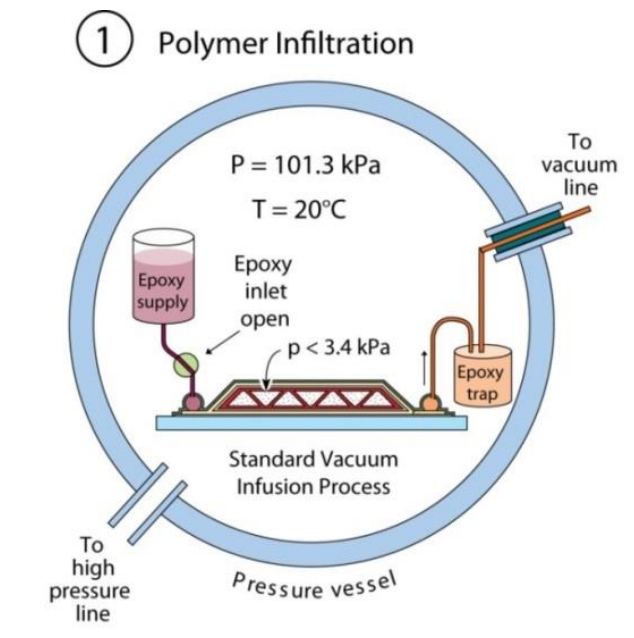

(2) Matrix Densification

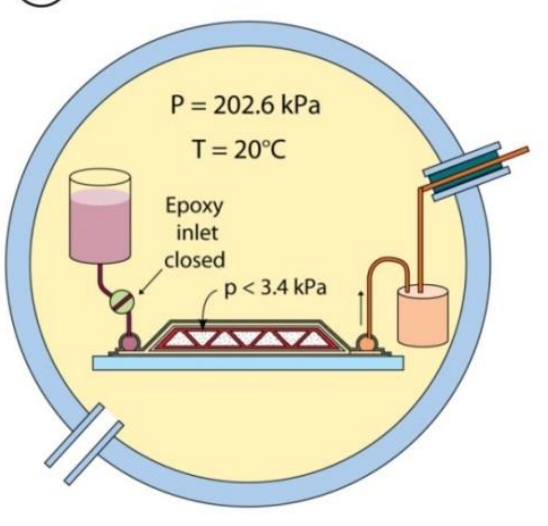

(3) Pressure Difference Translation

(4) Cure
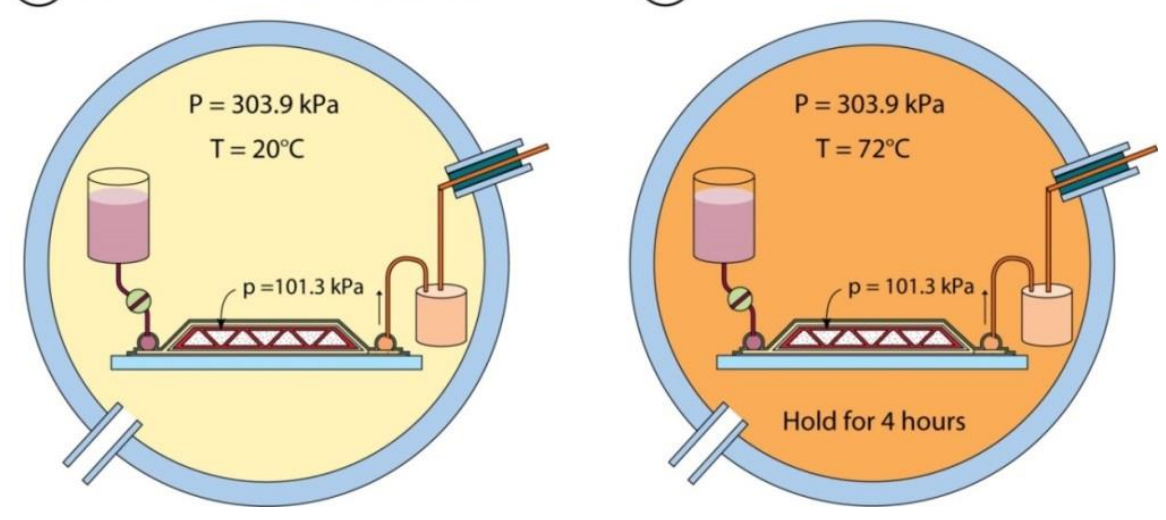

Figure 42: Illustration of the temperature and pressure cure process of the autoclave and vacuum bagged part. 


\subsection{Post-Cure Panel Fabrication}

After the panel is fully cured, it is removed from the autoclave for all post-cure panel fabrication steps. First, the infused panels must be removed from the vacuum infusion process materials including breather materials, distribution media, peel ply, and bag films. These materials are removed using an assortment of cutting, prying, and scraping methods. A diamond-tipped wet saw was used to cut the flat composite panels or corrugated structures into precise sizes to be used for quasi-static or dynamic testing, illustrated in Figure 43. Different sizes where required for testing in specific applications to span the needs of testing a single unit cell to a large blast panel $(610 \mathrm{~mm} \times 610 \mathrm{~mm})$. Based on the design, the size of a panel should not affect the mechanical performance. However, larger panels (specific to the blast panels) are more difficult to manufacture and may have more imperfections as a result.
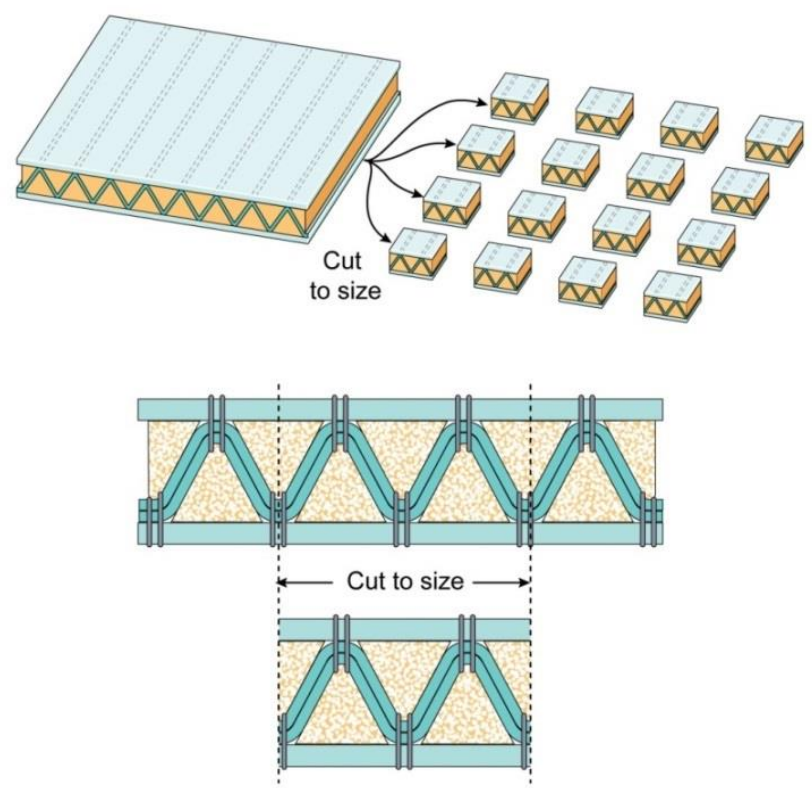

Figure 43: Cured composite structures are precisely cut into the desired shape for quasistatic or dynamic testing. The two-cell structures were used for laboratory dynamic impact testing. 
Multi-tier structures (used for high explosive blast testing) require a larger degree of postcure processing. As shown in Figure 44, the multi-tier sandwich structures are cut into $203 \mathrm{~mm}$ x $203 \mathrm{~mm}$ squares and mounted to an additional mounting plate to attach to the blast rig. The rear face mounting plate is made from S2-glass composite for the dynamic measurements using Kolsky Bars (to minimize the attenuation of stress waves) and either $6.35 \mathrm{~mm}$ (1/4 inch) aluminum or composite plates were used for vertical pendulum test samples. Aluminum plates were easier, quicker, and cheaper to use when viable. The baseplate was affixed to the core by polysulfide glue or the SC-11 epoxy used for the matrix. Both adhesives showed excellent ballistic shock response and proved very effective. In some panels and additional $12.7 \mathrm{~mm}(1 / 2$ inch) aluminum rigid front face was added to remove the effect of facesheet failure.

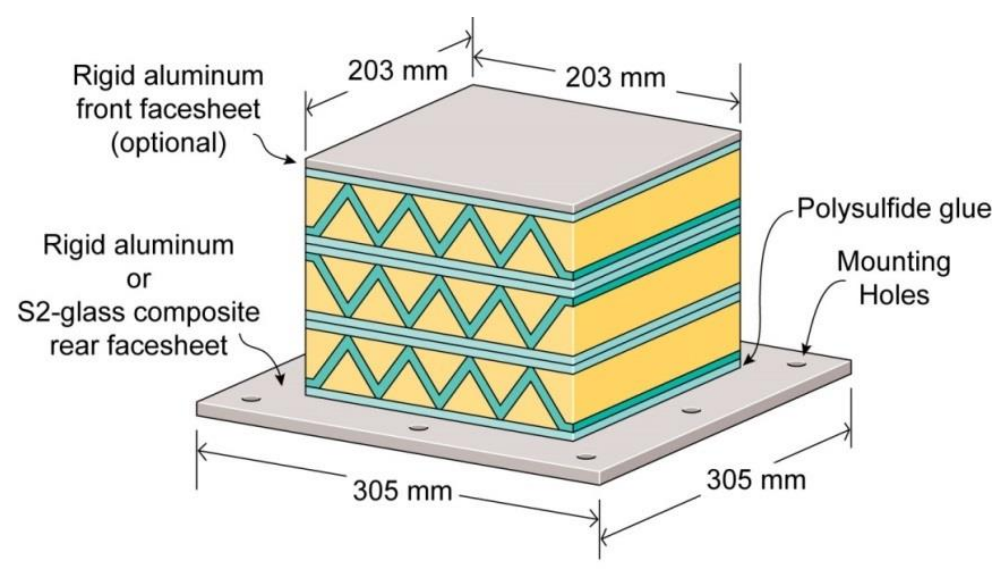

Figure 44: The multi-tier panel created for high explosive blast testing.

Finally, and perhaps the most difficult, was the construction of the open core sandwich structures. To assure a realistic comparison of the foam-filled and open corrugated cores, all structures where created with the foam inserts. During the infusion and cure process, core compression was observed, slightly altering the intended geometry of the structure. Rigid inserts were originally investigated for the open core structure to provide ease in removal, but the 
structures possessed sufficiently different geometry to add concern for response comparison. As a result, all panels were constructed with foam inserts and the foam was removed via mechanical methods. The use of drills, pneumatic hammers and pneumatic scrapers allowed the foam to be removed on a cell-by-cell basis. Every effort to minimize or eliminate any damage to the core web from the mechanical foam removal process was used. Metallic prismatic tooling was built to precisely fit into the prismatic volume created by the foam and only remove the unwanted foam. Visual inspection after foam removal occurred to find any matrix crazing resulting from the foam removal process. Samples exhibiting any visual damage were removed from the sample set. Pre-damaged struts are likely to provide reduced failure strengths and erroneous results through the geometrical change in core or nodal attachment, pre-developed matrix crack formation, or fiber damage.

\subsection{Manufacturing Imperfections}

Every panel manufactured was created by hand and imperfections were introduced in the sewing process, vacuum bagging and infusion, and post-cure fabrication. It was observed that manufactured samples exhibited slight imperfections from the as-designed glass fiber core sandwich panel, Figure 27. Unintended in the original design, the pressure differential created by the applied vacuum provided sufficient loading to the structure to slightly compress the foam core inserts resulting in non-flat facesheets, Figure 45(a). Limitation in the sewing process and precision yielded a $\pm 1 \mathrm{~mm}$ variation in the stitch placement. These factors created slight variations in node height and internode separation, Figure 45(b). Combined, these two factors 
created slight variations between strut lengths which created a significant difference (up to a seven times increase) in the quasi-static compressive modulus.

a)
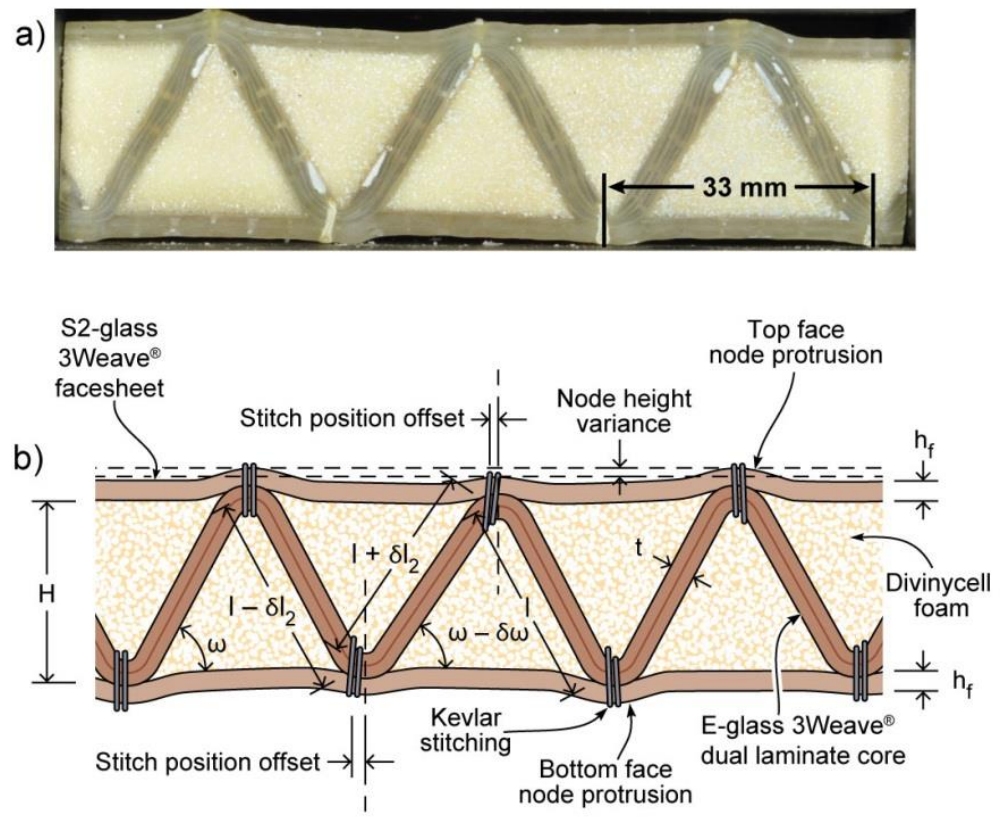

Figure 45: a) Photograph of an as-manufactured panel (Design 3) and b) schematic illustration of node height variations resulting from stitch offset during manufacture. Imperfections resulted in small variations of strut length, 1 , and strut orientation angle, $\omega$.

Imperfections created strut lengths that were measured to be $14 \pm 1 \mathrm{~mm}$ for design 1 and $25 \pm 1.5 \mathrm{~mm}$ for designs 2 - 4. Initial testing revealed these imperfections resulted in unequal strut loading at small strains, further discussed in Chapter 7. A series of single unit cell samples were therefore fabricated with equal length trusses and extra epoxy added to troughs in the wavy facesheets, Figure 47. This epoxy uniformly distributed the load to the entire sample surface and resulted in parallel facesheet surfaces. The mechanical response of both the "as manufactured" three cell core and the smoothed, facesheet single unit cell structures are both presented in this study. 


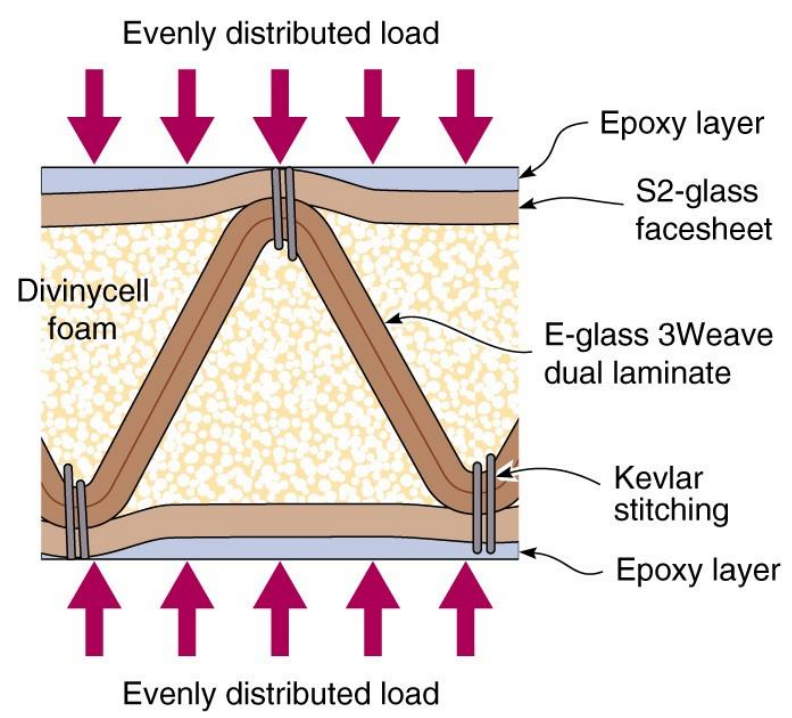

Figure 46: Unit cell manufactured with epoxy filler on the facesheets to minimize imperfections and uniformly distribute pressure.

The construction of the multi-tier sandwich panels was simply created by laminating the entire single tier sandwich structures together. The tolerance variations in nodal separation created by the precision limitations in the sewing process created nodal misalignments during the manufacture of the multi-tier structures. As seen in Figure 48, the single tier structures are intended to be aligned so the upper nodes of the lower tier structure are coincident with the lower nodes of the upper tier structure. Small nodal separation distance errors create nodal offsets that lead to non-ideal load transition. A single misalignment compounds the overall effect by creating misalignments through the remainder of the cells. The result of this imperfection is a nodal moment created during loading that alters the failure mechanism back from a stretchdominated failure mode to a bending-dominated failure mode. 


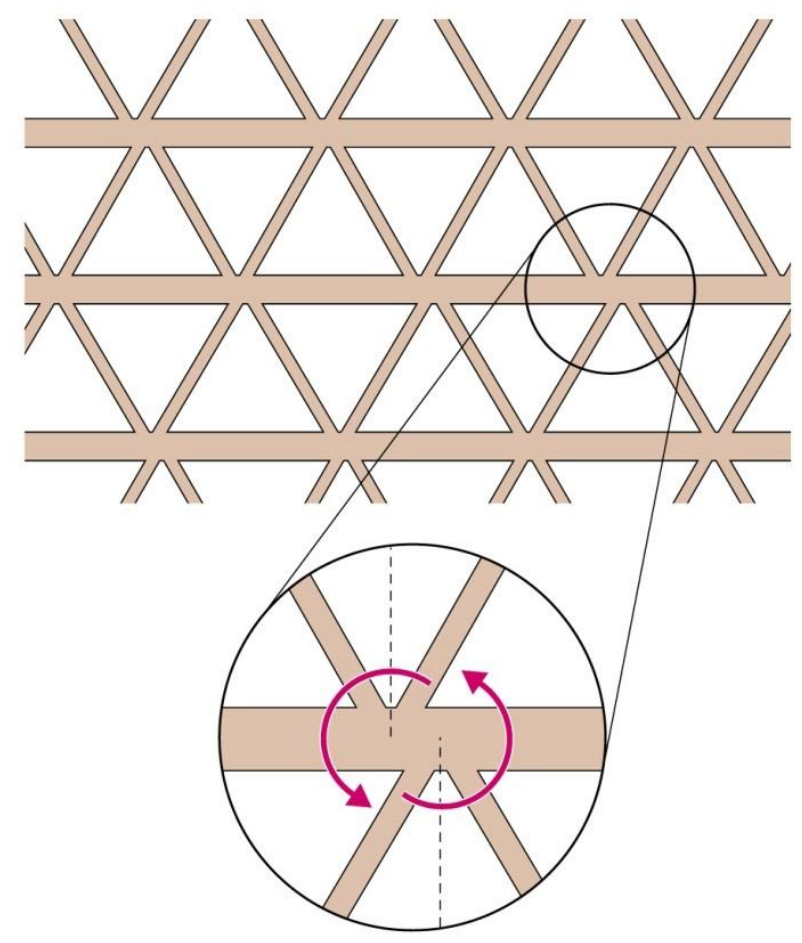

Figure 47: Manufacturing limitations create nodal misalignments throughout the multitier structure. These misalignments create moments around the node during loading and can alter the failure mode from a stretch-dominated design to bending-dominated. 


\section{Chapter 6}

\section{D Woven Composite Strut}

\section{Quasi-Static Loading}

The hybrid composite corrugated sandwich panel structure was designed for loading in both edge constrained and rear face supported boundary conditions. Analysis of the facesheets and strut components is critical for understanding failure mechanisms and the mechanical response for core design and future optimization. Loading in an edge-clamped scenario places the structure in bending, with components of both the core and face sheets placed in in-plane tension or compression. The loading on the structure, which uses a 3D laminated woven E-glass fiber fabric to form the internal core struts and a higher strength 3D woven S2-glass fiber fabric to provide tensile stretch resistance [111] in the face sheets, is illustrated on the component level in Figure 48. Studies of the quasi-static [73] and dynamic [37] compression of this structure indicate that the compressive strength of the struts is controlled by either elastic (Euler) or plastic fiber microbuckling $[50,112]$, and the use of 3D weaving can successfully eliminate both low strength interlaminar delamination and brooming mechanisms observed in laminated composite architectures [55, 100, 113]. Delamination failure is often observed in distinctly laminated composites where localized strain variations create shear stresses in excess of the shear strength of the matrix and cracks are formed. Cracks are easily propagated along the laminate interface 
and failure occurs. Brooming is a compressive failure mechanism observed at the contact points between a fiberous composite and loading surface. Slight variations in surface contact create localized areas of increased stress and the matrix will fail in compression or shear. Limited boundary constraints allow the fibers to deform laterally as cracks are produced, resulting in the matrix entirely failing and fibers buckling and deforming in all directions (similarly to a broom being compressed downward). Studies of the quasi-static in-plane tensile failure of 3D woven composites indicate failure through material yield, as equivalent to unidirectional composites [110]. The response of a laminated 3D woven fiber composite strut has yet to be investigated.

The failure mechanisms and mechanical properties of the laminated composite strut in axial tension and compression is investigated here.

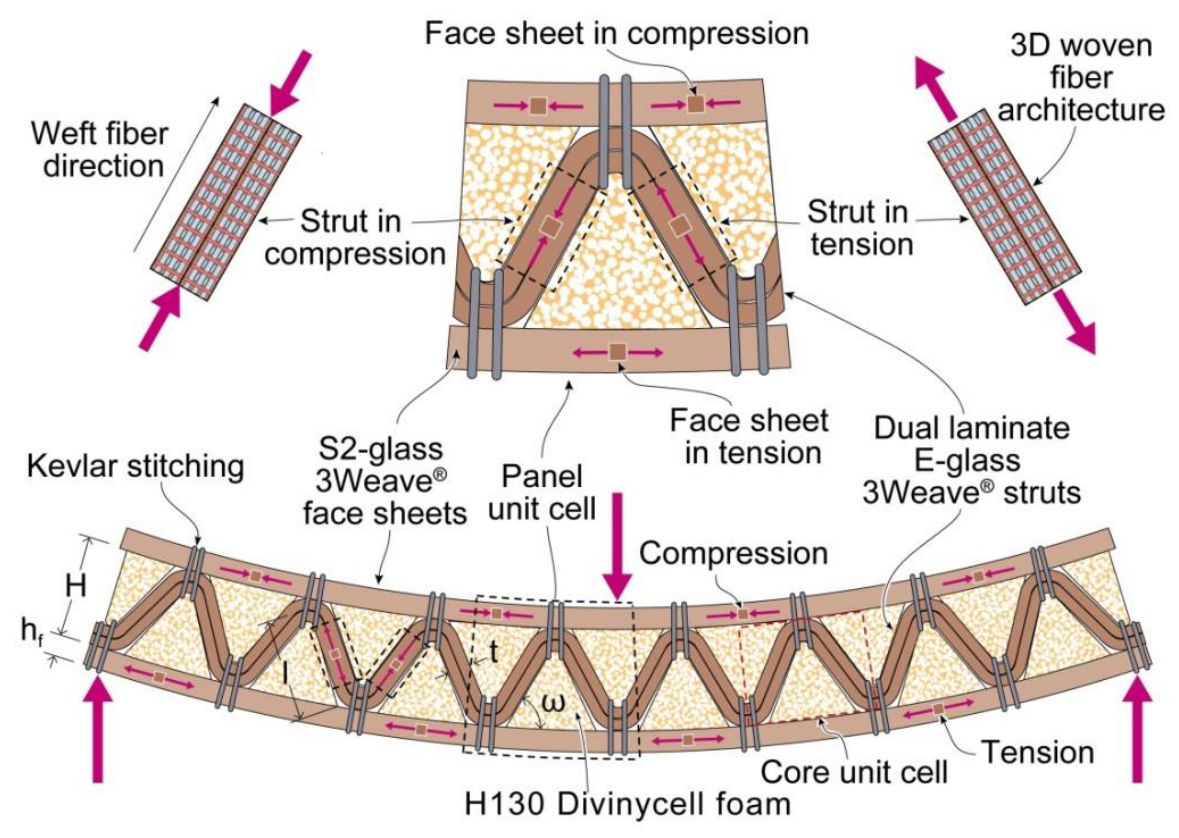

Figure 48: Schematic illustration of a hybrid polymer foam/corrugated composite core sandwich panel utilizing 3D woven E-glass fiber textile to create the core struts and S2- 
glass for the face sheets. When subjected to bending, the face sheets and core struts are loaded in either in-plane tension or compression.

The design of the composite sandwich panel requires an understanding of the failure mechanisms of the 3D woven E and S2-glass fiber composite components in tension and compression, the influence of z-pinning, and the effect of fiber volume fraction upon failure. To aid in the understanding of the structure and the mechanisms behind failure, characterization using both high resolution X-ray Computed Tomography (XCT) and optical microscopy techniques is presented. When combining the characterization with experimental testing, simplified micromechanical models are established to investigate the relation between strut geometry, fiber and matrix properties, composite fiber structure and the mechanical properties of the struts. All testing and analysis focuses on loading parallel to the weft fiber tows only to mirror the fiber/strut configuration of the quasi-static and dynamic structures in this research study.

\subsection{Characterization}

Characterization of the fiber architecture on both the E and S2-glass 3D woven composites is performed through use of X-Ray Computed Tomography (XCT) and optical microscopy to determine how the weave geometry differs from the ideal architecture as presented in Figure 34. Imperfections related to fiber tow tortuosity / waviness is expected given the difficulty and variability in both the weave process and composite manufacture. Fiber waviness can result in substantial initial fiber tow misalignment which adversely effects the compressive strength [114]. While many studies confirm a significant delamination resistance 
benefit of 3D woven textiles, the fiber waviness created within the in-plane warp and weft fiber tows increases susceptibility to kink band formation and microbuckling failure under in-plane compressive loading $[107,109,110]$. A kink band is localized zone of fibers failing by bending all oriented along a specific plane, Figure 49. Significantly influenced by the initial fiber misalignment angles, $\phi$, plastic and fractured kink band are commonly formed in sets (i.e. double kink band) under compressive and shear loading. This section quantifies the initial average fiber misalignment angles observed as a result of the fiber waviness for E and S2-glass 3D woven architecture specific to this structure that leads to kink band formation.

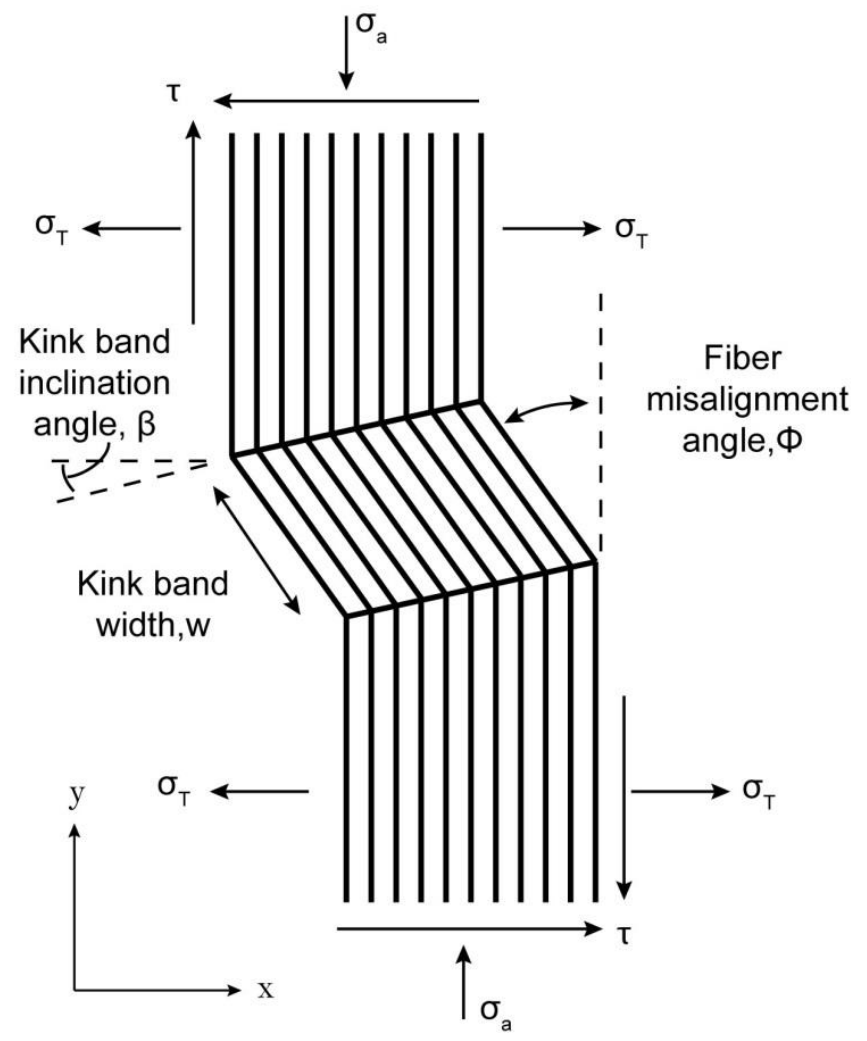

Figure 49: Kink bands are formed under axial compressive loading of fibers where a group of fibers locally bends along a plane inclined at angle $\beta$. Drastically influenced by the initial fiber misalignment angle, $\phi$, failure can result in the formation of plastic kink bands or even fractured kink bands. [114] 


\subsubsection{X-Ray Computed Tomography}

X-Ray Computed Tomography (XCT) allows for the 3D reconstruction of the fiber architecture through the thickness of the strut. Reconstruction is conducted using a $225 \mathrm{kV}$ microfocus XCT system with a flat panel PerkinElmer XRD 1621 X-ray detector at Chesapeake Testing (Belcamp, MD), the setup was maximized for low-energy, high resolution scanning in order to resolve the individual fibers and the effective tow waviness. The samples were scanned at $80 \mathrm{kV}$ with a tube current of $45 \mathrm{uA}$ yielding volumes capable of reconstruction with a final voxel size of $6 \mu \mathrm{m}$.

An XCT image of an E-glass single laminate strut is shown in Figure 50(a). The image shows warp and weft fiber tows that exhibit significant fiber tow waviness. In some cases, warp or weft tows that were stacked vertically during the weave process slid laterally relative to one another and impinged upon neighboring transversely oriented tows. Weft tow waviness appears to predominantly be resulting from the impingement of contact points of the z-yarn with the weft tows. The warp tows are, in turn, deflected by the misshaped weft fiber tows. Rectangular shaped resin rich pockets are created in regions where no fiber tows were placed or laterally slid out of alignment during manufacture, Figure 50 (b) and (c). Detailed analysis of the XCT images, Figure 50, shows that parallel z-yarns are offset in their pseudo-sinusoidal profile by approximately $5.2 \mathrm{~mm}$ (the thickness of a weft tow). This effect is illustrated in Figure 34, which creates a weft tow misalignment that varies through the width of the strut. This offset can be advantageous since the average tow misalignment in the width direction will vary, thereby minimizing a band of fiber tows through the width direction exhibiting a large initial average fiber tow misalignment. 

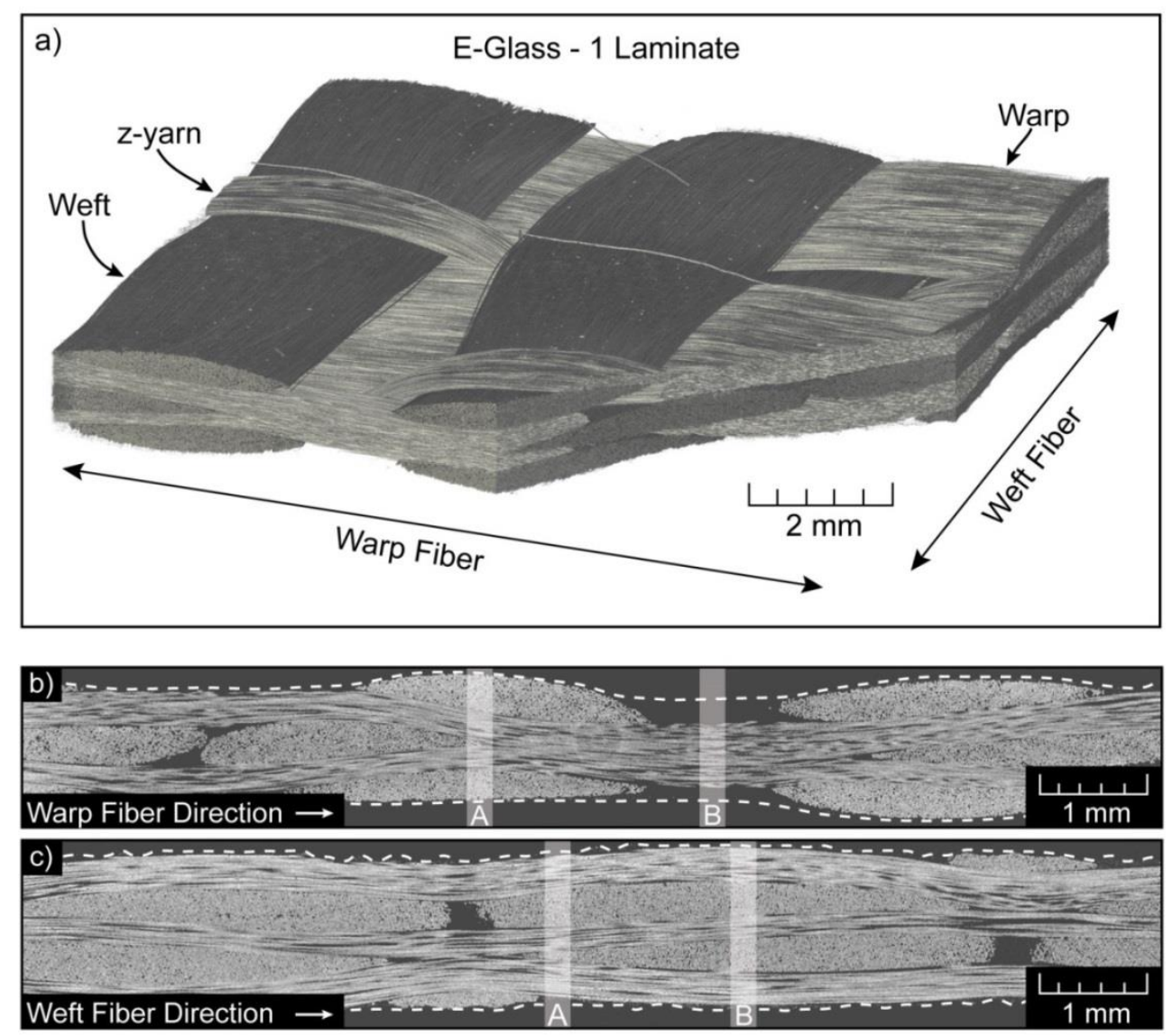

Figure 50: XCT images of a single laminate E-glass strut. (a) The 3D structure illustrates maximum fiber waviness at the point the z-yarn tows contacts the weft fiber tows. Cross sectional slices show the (b) warp and (c) weft fiber tows respectively. The dotted lines illustrate the epoxy surface location and the vertical bands $A$ and $B$ indicate planes for which tow misalignment data is presented. This structure is void free and internal void areas are filled with epoxy.

Measurements of the warp and weft fiber tow misalignment at planes A and B revealed misalignments angles observed both through the thickness and width of strut. The initial average fiber misalignment angle varied significantly as a function of position along the length in both the warp and weft fiber tows. Figure 51 provides a histogram of measured misalignment angles from the XCT images along planes A and B for a single laminate E-glass strut. The positions were chosen to represent both the extremes of both straight and wavy positions 
observed within the fiber tows ${ }^{4}$. The warp fiber tows exhibit greatest fiber waviness along plane A where the slippage of the center weft fiber tow (ultimately from z-yarn compressive loading) creates a space for the warp fiber tows to deform and occupy that space. Misalignment at Position $\mathrm{A}$ is observed to range from $-9.3^{\circ}$ to $7.1^{\circ}$ with an average misalignment of $-1.8^{\circ}$. Position B is straighter with measured misalignment ranging from $-1.9^{\circ}$ to $5.3^{\circ}$ with an average misalignment of $1.6^{\circ}$. The weft tow misalignment was greatest at the point adjacent to the $\mathrm{z}$-yarn loading on the weft fiber tow with an observed range from $-3.7^{\circ}$ to $11.2^{\circ}$ with an average misalignment of $1.5^{\circ}$ for Position A. Position B was well balanced with extremes ranging from $3.2^{\circ}$ to $4.0^{\circ}$ with an average misalignment of $-0.3^{\circ}$.

\footnotetext{
${ }^{4}$ Misalignment angles presented are defined as a positive angle when the fiber tow exhibits a positive slope and negative when the fiber tow exhibits a negative slope (with respect to the horizontal axis).
} 

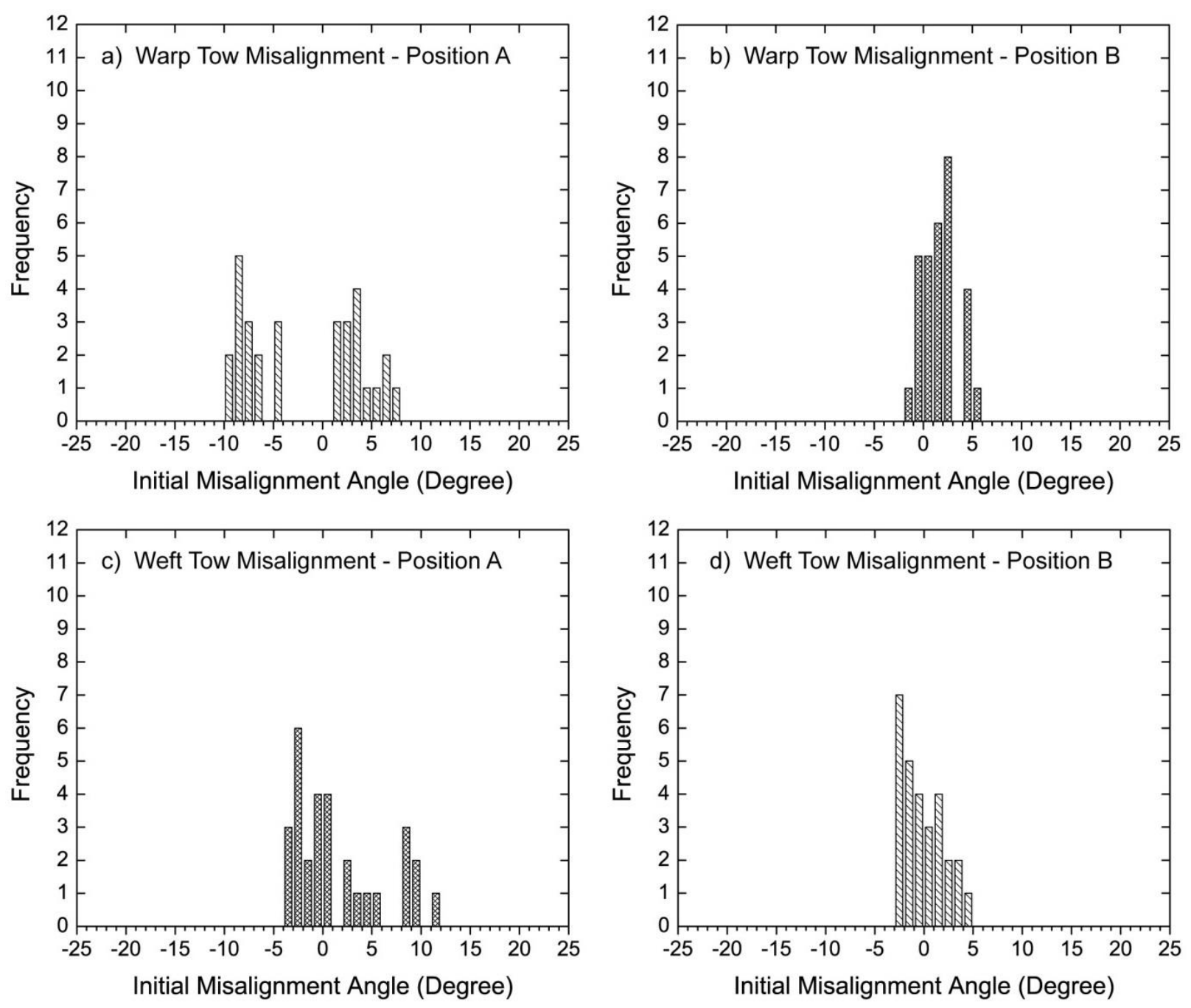

Figure 51: Initial fiber tow misalignment angles are measured for a single laminate E-glass composite strut. Warp fiber tow misalignment angles are provided for positions (a) adjacent to a "mid-weft" tow (Position A, Figure 4b) and (b) furthest position away from the weft tow (Position B, Figure 4b). Weft fiber tow misalignment angles are provided for positions (c) adjacent to the z-yarn (Position A, Figure 4c) and (d) "mid-warp" tow position (Position B, Figure 4c). These positions provide the relative extremes observed in the fiber waviness along the length of the sample.

Similarly, XCT analysis was performed on 2 and 3 laminate versions of the 3D woven fiber geometry to reflect the sandwich structure geometry. The laminated versions exhibited near identical fiber waviness as a single laminate. The greatest fiber waviness was observed at 
the positions of z-yarn impingement. Manufacturing variability in the laminate process greatly affected the positioning of tows vertically, but the sliding was observed due to the looseness of the z-yarn and, thereby the inability to maintain the desired fiber architecture. Figure 52 illustrates XCT images of the 2 and 3 laminate E-glass composites. A summary of the misalignment angles for the S2 glass composite struts is given in Table 5.

Table 5: Measured initial average fiber misalignment angles throughout the warp and weft tows in single laminate struts as measured with XCT imaging corresponding to Figures 4 and 7 for $E$ and S2, respectively. This study reveals the peak misalignment angles do not primarily control the critical failure strength and an average is a better representation of the overall tow misalignment.

\begin{tabular}{|c|c|c|c|c|c|}
\hline \multirow{2}{*}{ Material } & \multirow{2}{*}{$\begin{array}{l}\text { Tow } \\
\text { Type }\end{array}$} & \multirow{2}{*}{$\begin{array}{l}\text { Measurement } \\
\text { Position }\end{array}$} & \multicolumn{3}{|c|}{ Tow Misalignment Angle (Degree) } \\
\hline & & & Minimum & Maximum & Average \\
\hline \multirow{4}{*}{ E-Glass } & \multirow{2}{*}{ Warp } & A & -9.3 & 7.1 & -1.8 \\
\hline & & $\mathrm{B}$ & -1.9 & 5.3 & 1.6 \\
\hline & \multirow{2}{*}{ Weft } & A & -3.7 & 11.2 & 1.5 \\
\hline & & B & -3.2 & 4.0 & -0.3 \\
\hline \multirow{4}{*}{ S2-Glass } & \multirow{2}{*}{ Warp } & A & -2.5 & 2.4 & 0.2 \\
\hline & & B & -10.5 & 14.9 & 1.5 \\
\hline & \multirow{2}{*}{ Weft } & A & -22.4 & 20.5 & 1.6 \\
\hline & & B & -3.0 & 7.0 & 1.1 \\
\hline
\end{tabular}




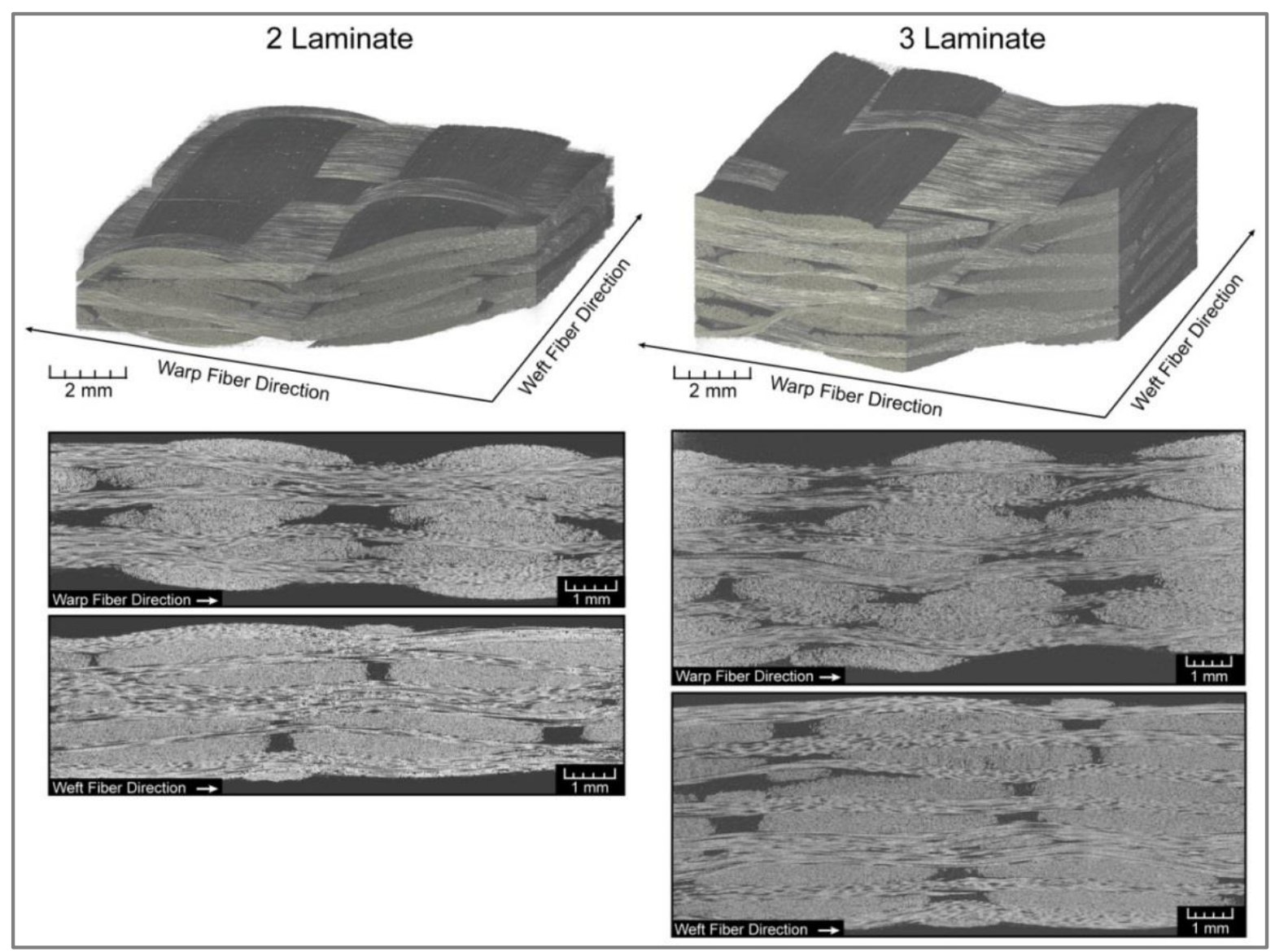

Figure 52: XCT images of a 2 and 3 laminate E-glass strut illustrate similar fiber waviness as a single laminate E-glass strut. Cross sectional slices show the warp and weft fiber. This structure is void free and internal void areas are filled with epoxy.

XCT images of the S2-glass fiber composite strut samples are shown in Figure 53. The S2-glass fiber architecture was maintained closer to the intended configuration with both the warp and weft tows in vertical alignment throughout the structure. Fiber waviness was imparted at the point of z-yarn impingement greatly affecting the initial average tow misalignment angle. In fact, while generally the tows are overall straighter (when evaluated over the length of the strut) the misalignment angles through the thickness direction can be large at the points of z-yarn 
impingement. Similar to the E-glass, an offset in the pseudo-sinusoidal z-yarn pattern is observed.
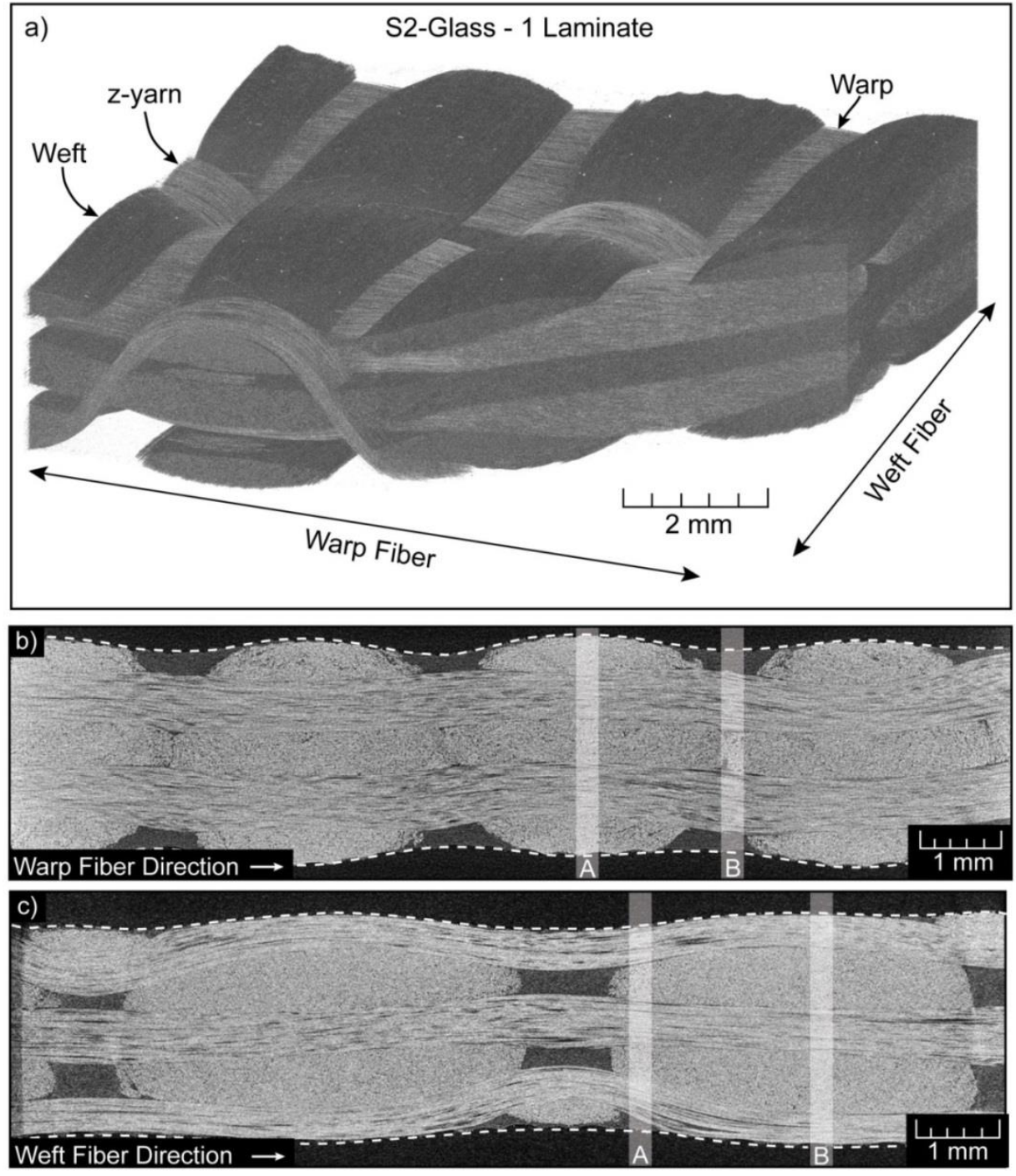

Figure 53: XCT images of a single laminate S2-glass strut. (a) The 3D structure illustrates maximum warp and weft fiber tow waviness near the point where the z-yarn tow contacts the weft fiber tows. Cross sectional slices containing the (b) warp and (c) weft fiber directions. The dotted lines illustrate the epoxy surface location and the vertical bands A and $B$ indicate planes for which tow misalignment data is presented. This structure is void free and internal void areas are filled with epoxy. 
Measurements of the warp and weft fiber tow misalignment for the S2-glass laminate at planes A and B were taken to reveal misalignments angles observed both through the thickness and width of strut. Figure 54 provides a histogram of measured misalignment angles from the XCT images along planes A and B for a single laminate S2-glass strut. The positions were chosen to represent both the extremes of both straight and wavy positions observed within the fiber tows. The warp fiber tows exhibit greatest fiber waviness along plane A where the slippage of the center weft fiber tow (ultimately from z-yarn compressive loading) creates a space for the warp fiber tows to deform and occupy that space. Misalignment at position A within the warp fiber tows is relatively straight and observed to range from $-2.5^{\circ}$ to $2.4^{\circ}$ with an average misalignment of $0.2^{\circ}$. Position B exhibits greater waviness ranging from $-10.5^{\circ}$ to $14.9^{\circ}$ misalignment with an average of $1.5^{\circ}$. The weft tow misalignment was greatest at the point adjacent to the z-yarn loading on the weft fiber tow with an observed range from $-22.4^{\circ}$ to $20.5^{\circ}$ with an average misalignment of $1.6^{\circ}$ for position A. Position B was well balanced with extremes ranging from $-3.0^{\circ}$ to $7.0^{\circ}$ with an average misalignment of $1.1^{\circ}$. A summary of the misalignment angles for the S2-glass composite struts is given in Table 5. 

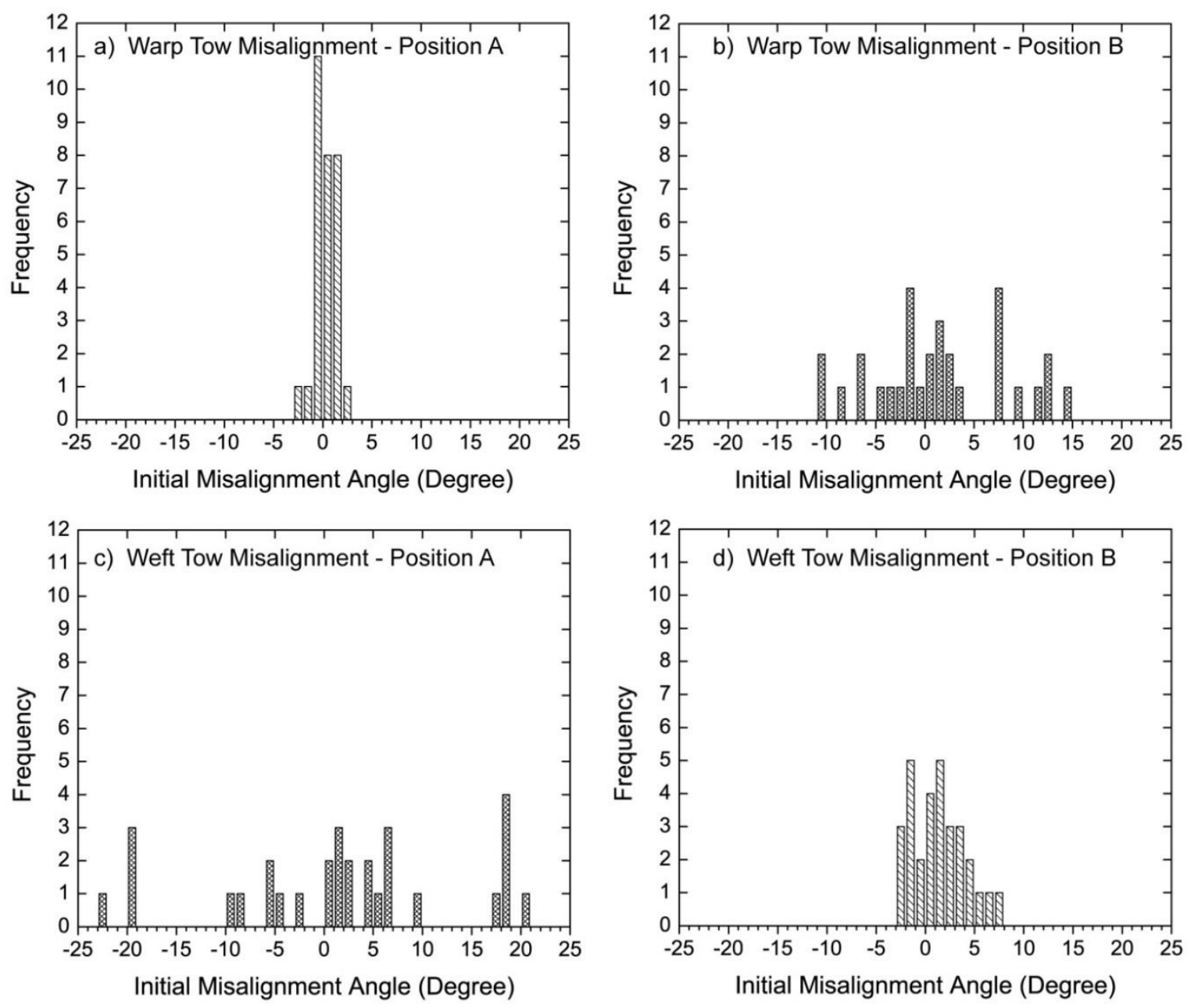

Figure 54: Initial fiber tow misalignment angles are measured for a single laminate S2glass composite strut. Warp fiber tow misalignment angles are provided for positions (a) adjacent to a "mid-weft" tow (Position A, Figure 7b) and (b) furthest position away from the weft tow (Position B, Figure 7b). Weft fiber tow misalignment angles are provided for positions (c) adjacent to the z-yarn (Position A, Figure 7c) and (d) "mid-warp" tow position (Position B, Figure 7c). These positions provide the relative extremes observed in the fiber waviness along the length of the sample.

\subsubsection{Optical Microscopy}

A series of optical micrographs of the E-glass 3 Weave ${ }^{\circledR}$ composite, Figure 55, provide higher resolution characterization of the structure. To obtain optical micrographs, the composite samples were cut and polished along planes parallel to the warp and weft fiber tows using a 
similar procedure to that described by Bartosiewicz et al. [115] and Sawyer et al. [116]. Optical micrographs were obtained using a Nikon Epishot inverted microscope equipped with a Nikon D-90 digital camera and a Hirox KH-7700 digital microscope. The higher resolution micrographs, Figure 55, confirm that both the warp and weft fiber tows in the E-glass composites have significant local variations in fiber waviness. Both the horizontal warp tows and horizontal weft tows exhibit waviness resulting from out of plane z-yarn compression. This z-yarn impingement creates the greatest misalignment in the weft tows.

The micrographs also confirm the presence of small resin pockets between the impregnated tows as a consequence of the fiber architecture. These pockets are an inherent result of the fiber architecture. All fibers in a given weft tow are observed to be oriented in the ideal configuration with the fiber cross section perpendicular to the intended axis of loading. The fiber volume fraction within a tow is higher than that of the overall composites fiber volume fraction. The maximum fiber volume fraction of a single tow was measured to be approximately $76 \%$, but locally can be lower due to non-optimal fiber packing. The diameter of the E-glass fibers was measured at approximately $18 \mu \mathrm{m} \pm 4 \mu \mathrm{m}$ and approximately 2,300 fibers comprise a weft tow. 

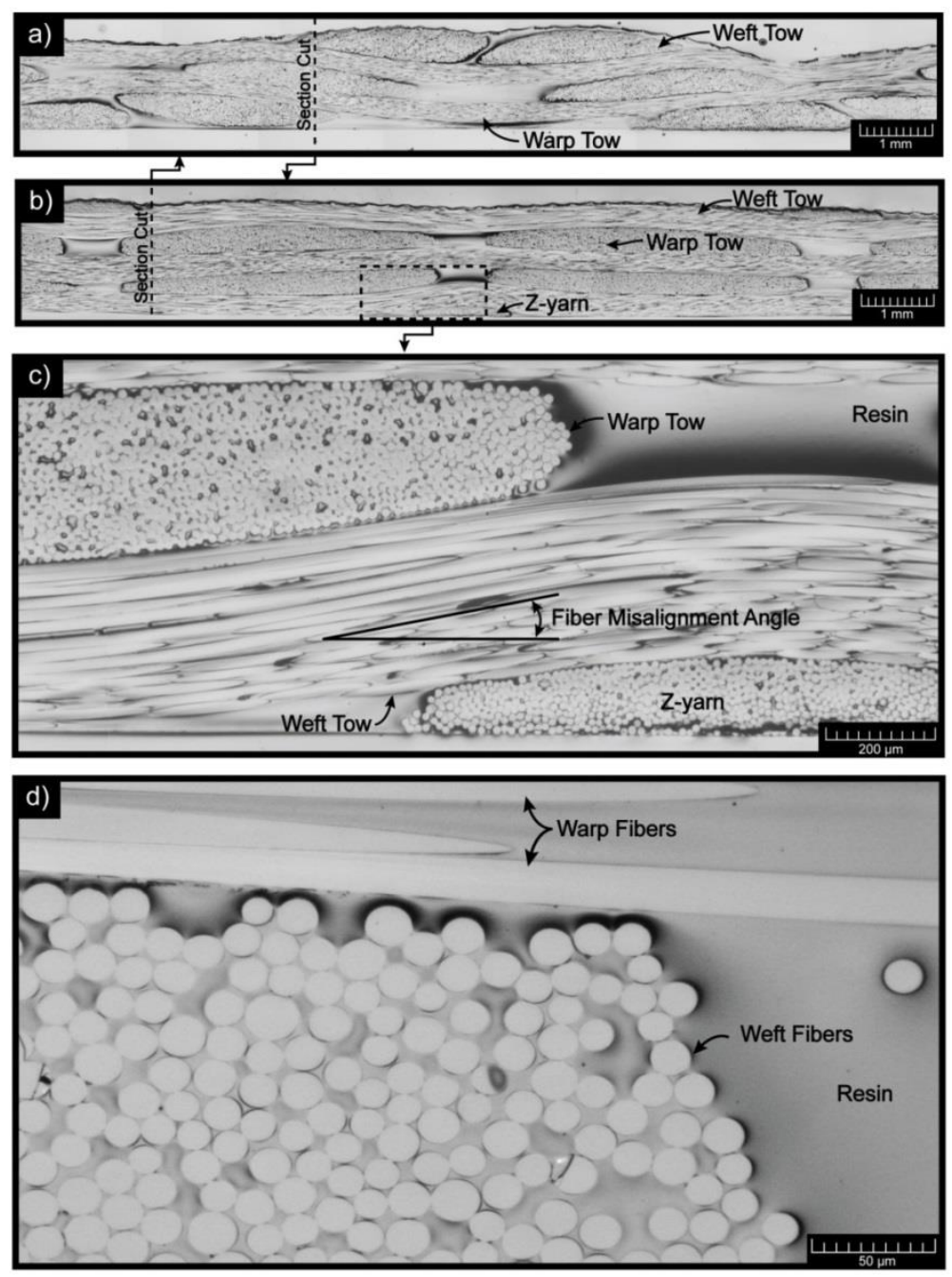

Figure 55: Optical micrographs of the E-glass 3 Weave ${ }^{\circledR}$ composite. Both the horizontal warp tows (a) and horizontal weft tows (b) exhibit waviness resulting from out of plane $z$ yarn compression. (c) Weft tow misalignment was greatest at the position of $z$-yarn impingement. (d) The diameter of the E-glass fibers was $18 \mu \mathrm{m} \pm 4 \mu \mathrm{m}$ for the approximately 2,300 fibers within a weft tow.

The optical micrographs of the S2-glass 3D woven architecture, Figure 56, indicate several distinct differences from the E-glass counterpart. First, the z-yarn holds the weft fiber tows in good vertical alignment, which in turn holds the warp fiber tows in good alignment. The greater alignment precision maintains the warp and weft town in a straighter orientation along the length. However, the closer magnification highlights the extreme misalignment that the zyarn places on the outer weft fiber tows. Misalignment is due to the tension of the z-yarn and 
highlights both the advantages and disadvantages that z-yarn tension provides. The micrographs also reveal the resin pockets between the impregnated tows similar to the E-glass counterpart.

The diameter of the $\mathrm{S} 2$-glass fibers used in construction measures $9 \mu \mathrm{m} \pm 1 \mu \mathrm{m}$ and approximately 8,000 fibers are observed within a weft tow.
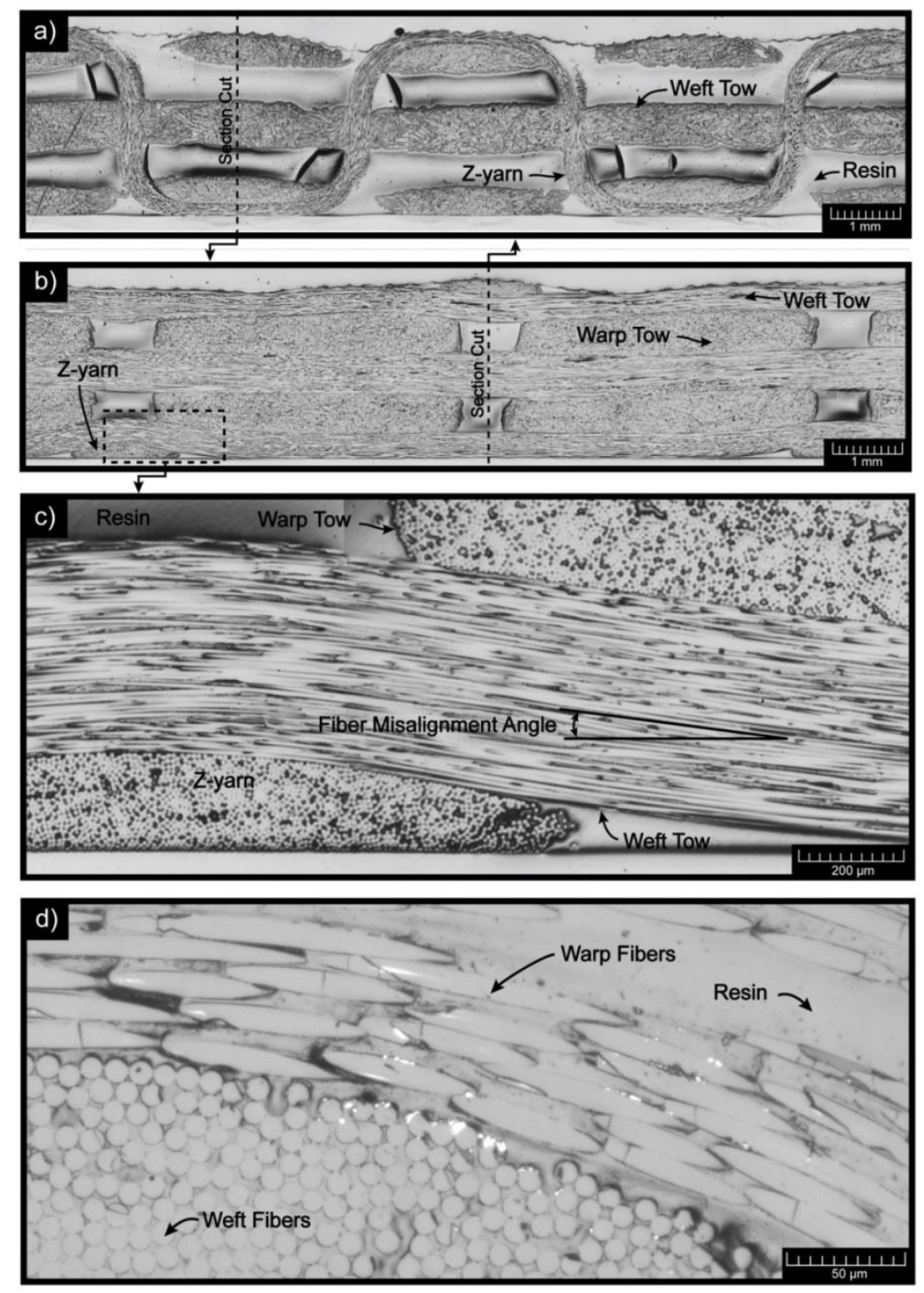

Figure 56: Low magnification of optical micrographs of the S2-glass $3 W e a v e^{\circledR}$ composite illustrate the (a) z-yarn square weave binding the weft fiber tows, (b) the misalignment of the horizontal weft tows resulting from the localized pressure applied by the binding zyarn, (c) weft tow misalignment near a z-yarn contact point, and (d) the diameter of the S2glass fibers was $9 \mu \mathrm{m} \pm 1 \mu \mathrm{m}$ for the approximately 8,000 fibers within a weft tow. 


\subsection{Tension}

The tensile response of 3D woven composites can be very well estimated from an isostrain model [110] commonly associated with unidirectional composites. For long-fiber, unidirectional, plastic composites loaded in tension in the fiber direction, the modulus is reasonably well predicted by the Voigt upper predictive bound while the Reuss relation provides a lower predictive bound by modeling the system as a transversely loaded unidirectional composite [25]. The Hashin-Shtrikman model provides a more precise bounding envelope for materials with dissimilar Poisson's ratios and converges to the rule-of-mixtures prediction when the Poisson's ratios of the fiber and matrix are equal [25]. The tensile strength is reached when either the fiber or matrix reaches their material specific ultimate tensile strength, thereby initiating failure. Failure in a portion of the strut allows for stress concentrations to build and damage to progress to full catastrophic failure $[22,117]$. It is predicted that this laminated 3D woven composite will behave in a similar manner as the z-yarn does not interfere when axial loaded in tension and the 3D woven composite is analogous to a $2 \mathrm{D}$ laminated composite.

\subsubsection{Test Methodology-Strut Tension}

The 3D woven composite struts were tested in tension in the direction parallel to the weft tow direction. Tensile tests conformed to ASTM D3039, with each test coupon cut to a length of $250 \mathrm{~mm}$ and a width of $25.4 \mathrm{~mm}$. Tabs utilized for gripping were beveled at approximately $15^{\circ}$ and epoxied to the gripping surfaces of the sample to prevent coupon damage from the compressive loading by the wedge action grips. Retro-reflective tabs were mounted on each 
sample to measure strain within the $150 \mathrm{~mm}$ gauge length of the test coupons at a strain rate of $10^{-3} \mathrm{~s}^{-1}$ at room temperature $\left(25^{\circ} \mathrm{C}\right)$. Figure 57 illustrates the tensile coupon set-up.

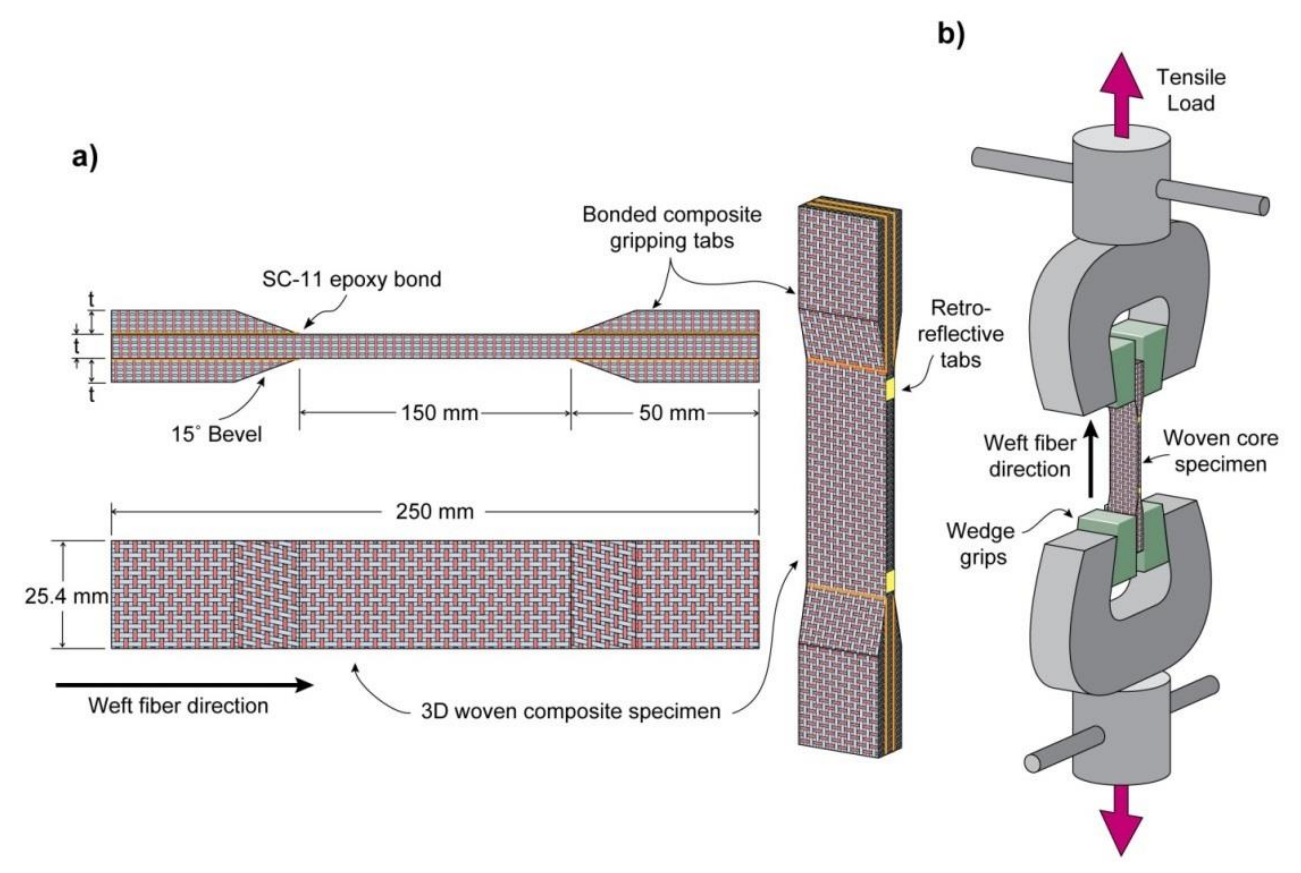

Figure 57: Tensile test coupons of the 3D woven composite. a) Samples where cut to length and bonded to gripping tabs to prohibit stress concentrations. b) The specimen was clamped in wedge action compression grips with the weft fiber direction oriented parallel to the axis of loading.

\subsubsection{Experimental Results-Strut Tension}

The measured tensile (Young's) modulus and tensile fracture strengths in the weft fiber direction are shown in Figure 58 and Figure 59 as a function of fiber volume fraction. The Young's modulus of the E-glass composite samples varied from 12 to $17 \mathrm{GPa}$ for fiber volume fractions of $30-54 \%$, while the S2-glass struts varied from 16-19 GPa for fiber fractions of $40-52 \%$. Within the scatter of the individual measurements, the modulus of the both the E-glass and S2glass exhibited a linear dependence upon fiber fraction of the strut. For comparison purposes, 
predictions for the tensile modulus and strength are plotted in Figure 58and Figure 59. The predictions are discussed in Section 5.2.3.

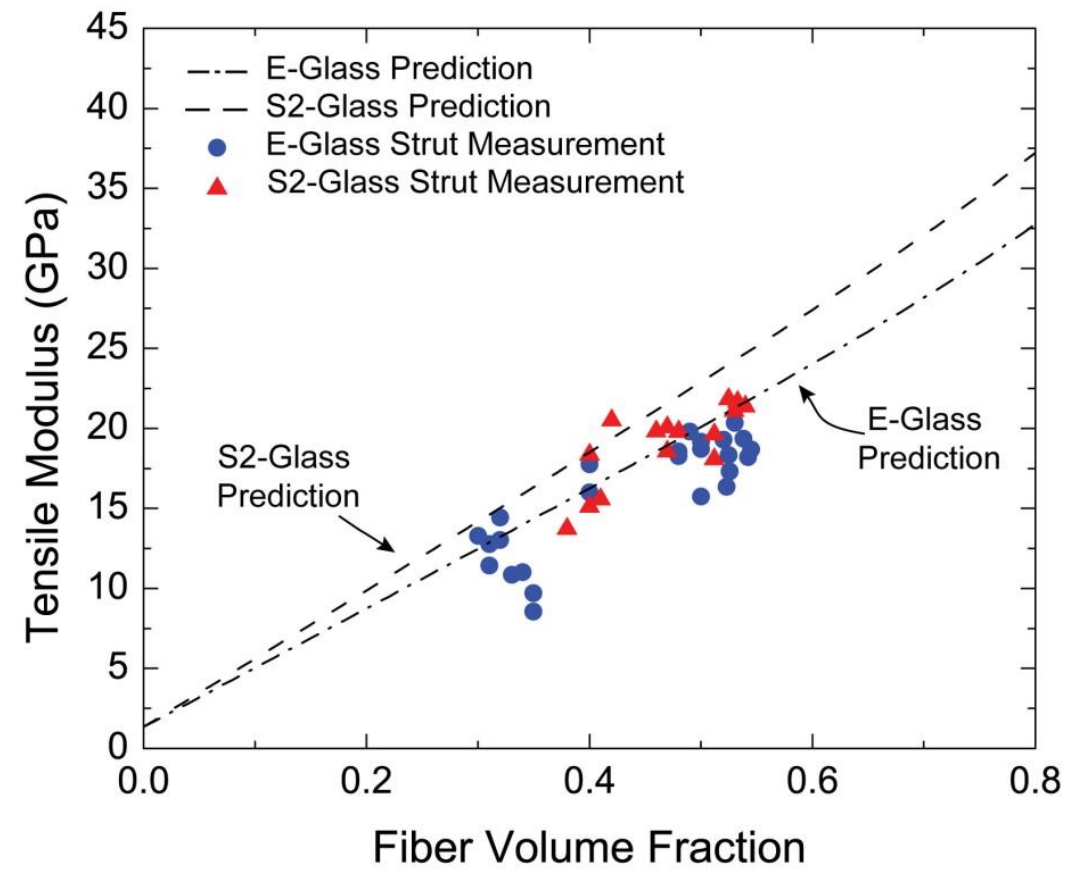

Figure 58: The fiber volume fraction dependence of the tensile modulus for $\mathbf{E}$ and S2-glass composite struts. Predictions use the experimentally measured modulus of the post-woven silane coated fibers which closely matches reported virgin fiber properties.

The tensile strength of the E-glass and S2-glass exhibited an approximately linear relation with fiber volume fraction, but their slopes were different, Figure 59. The average strength of the E-glass composites varied from 210 to $425 \mathrm{MPa}$ for fiber volume fractions of 30-54\% while the S2-glass composite strength increased from 405 to $650 \mathrm{MPa}$ as the fiber fraction increased from 40 to $52 \%$. 


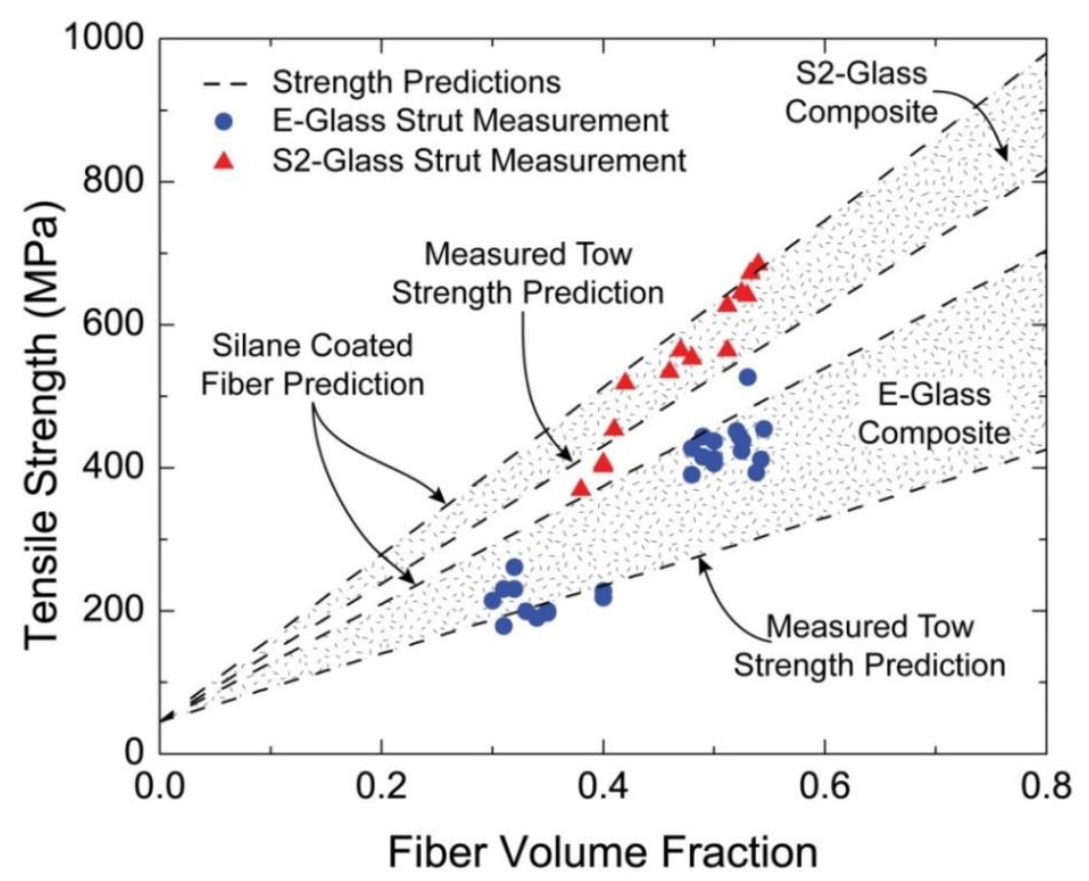

Figure 59: The fiber volume fraction dependence of the tensile strength for the $E$ and S2glass composites. Model predictions, Equation 6.4, are bounded using the theoretical strength of a silane coated fiber and the experimentally measured strength of the postwoven fiber tow rovings.

\subsubsection{Analytical Predictions-Strut Tension}

The tensile stiffness and strength of 3D woven composites has been previously modeled by Cox et al. using an iso-strain approach [109]. The mass of the z-yarn fiber is assumed negligible and delamination failure is assumed inoperative with this model. Loading is taken to be oriented parallel to the axial (weft) fibers and perpendicular to the transverse (warp) fibers. The upper bound modulus prediction of the composite, $E_{c}$ is given by;

$$
E_{c}=E_{\text {weft }} f_{\text {weft }}+E_{\text {warp }}\left(1-f_{\text {weft }}\right)
$$

where $f_{\text {weft }}$ is the fractional area of axial (weft) fibers in the composite, $\mathrm{E}_{\text {weft }}$ is the modulus of the weft fiber occupied area, and $\mathrm{E}_{\mathrm{warp}}$ is the modulus of the warp fiber occupied area. Ignoring 
the z-yarn component $f_{\text {weft }}$ can be calculated from the data provided by assuming that the fiber fraction in the weft and warp fiber occupied areas are equivalent to the overall fiber fraction of the composite. This gives $f_{\text {weft }}=0.498$ or 0.519 for $\mathrm{E}$ and $\mathrm{S} 2$-glass composites respectively. This assumption is supported by the visual approximation that the fraction of all fibers in the weft direction is equivalent to the fraction of the area occupied by the weft tow laminates on a section normal to the load axis (as compared with the warp fibers and warp tow laminate area).

The modulus of the weft fiber occupied region is given by;

$$
E_{\text {weft }}=E_{f} v_{f}+E_{m}\left(1-v_{f}\right)
$$

while the modulus of the warp fiber occupied area is given by;

$$
E_{\text {warp }}=\left[\frac{v_{f}}{E_{f}}+\frac{\left(1-v_{f}\right)}{E_{m}}\right]^{-1}
$$

where $\mathrm{E}_{\mathrm{f}}$ is the modulus of the glass fiber, $\mathrm{E}_{\mathrm{m}}$ is the modulus of the matrix, and $v_{\mathrm{f}}$ is the overall fiber fraction of the composite

However, 3D woven composites contain sufficient fiber waviness to reduce the modulus under initial loading [107]. In tension, the measured modulus will approach the predicted modulus as the fiber tows straighten, provided that the modulus of the fiber is significantly higher than the matrix and the matrix is sufficiently compliant to preclude matrix failure before the fiber tows straighten. While highly dependent upon the materials and manufacture method of the weave, Cox et al. predicted a 10-20\% higher modulus than experimental measurements for heavily compacted samples of similar weave geometry [109]. 
Using the measured modulus of the E-glass and S2-glass fibers, the predictions of Equation (6.1) are compared with experimental data in Figure 58. The measured tensile modulus of the E-glass and S2-glass fiber composites are within $10 \%$ of the predictions and consistent with the arguments of Cox et al. that an empirical knockdown parameter is needed to account for fiber waviness effects.

The tensile strength of a composite loaded in the weft tow direction, $\sigma_{c}$, can be estimated by assuming the weft fibers and matrix are equally strained at failure. The warp and z-yarn fibers are assumed to have a negligible effect in tension [110] as the critical strength of a high strength fiber/epoxy system operating in series is limited by the strength of the matrix which, typically, exhibits a much lower tensile strength than the fiber. This leads to a rule-of-mixtures predicted strength given by;

$$
\sigma_{c}=\sigma_{f} v_{f A}+\sigma_{m}\left(1-v_{f A}\right)
$$

where $v_{\mathrm{fA}}$ is the fiber fraction of only the axial (weft) fibers in the composite, $\sigma_{\mathrm{f}}$ is the fiber strength, and $\sigma_{\mathrm{m}}$ is the matrix strength. The strength prediction, Equation 6.4, is bounded using either the silane coated fiber (ideal strength) or experimentally measured tow strengths of postprocessed woven rovings (lower limit), and plotted on Figure 59 for both the E-glass and S2glass fiber composites and compared with the experimental data for both fibers. The experimental data for the composites should exist between the predicted bounds. Small imperfections due to the manual manufacturing process of each test coupon can create slight fabric misalignment $\left(<1^{\circ}\right.$ offset $)$ and small variations in the bonding of the tabs to include bevel variations of up to 5 degrees and thickness variations in bonding interface. These imperfections are hypothesized to create some scatter in the data. 


\subsection{Compression}

Compressive loading in the fiber direction is more complicated since a variety of failure modes including global buckling, inter-ply delamination (splitting), brooming, and fiber microbuckling [114] can be activated. The weak delamination and brooming failure modes of axially compressed unidirectional and $0^{\circ} / 90^{\circ}$ laminated composites can be eliminated through the redesign of the fiber architecture by incorporating "binding" fibers oriented transverse to fiber ply plane [107]. The hypothesis is that the laminated 3D woven fiber composite will also exhibit resistance to delamination. Fiber microbuckling then dominates the response, and the matrix shear strength and fiber misalignment angle control the compressive failure strength of the composite, leading to a situation where the fiber strength is predicted to have no effect upon the compressive strength [118]. The effect of the fiber material along with the thickness to length ratio (t/l) and fiber volume fraction is investigated.

\subsubsection{Test Methodology- Strut Compression}

The compressive strength of the E and S2-glass composites has been measured parallel to the weft fiber tow direction. The panels tested in compression used a Combined Loading Compression (CLC) test fixture and followed the procedure defined in ASTM D6641. Test specimens were cut to a length of $152.4 \mathrm{~mm}$ parallel to the weft direction and tested with a gauge length of $25.4 \mathrm{~mm}$. A laser extensometer was used to measure the gauge section strain through use of retro-reflective tabs placed at the gauge section length limits. 

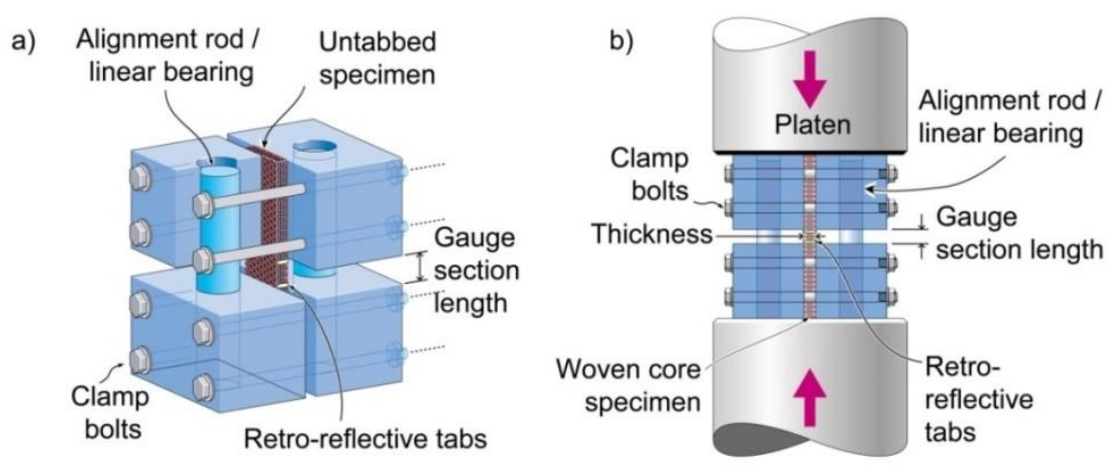

Figure 60: The Combined Load Compression (CLC) rig was used for confinement of the composite samples. a) The test specimen was cut to length and clamped in the CLC fixture. b) The weft fiber tows were aligned parallel the axis of loading.

\subsubsection{Experimental Results - Strut Compression}

Typical stress-strain responses for E-glass struts constructed from 1, 2, and 3 laminates (nominal $\mathrm{t} / 1$ ratios of $0.07,0.12$, and 0.18 respectively) with fiber fractions between 54 and $56 \%$, are shown in Figure 61. Failure occurring within the gauge length allowed for a successful test, while failure within the grip region was determined to be invalid. Only two types of failure was observed, microbuckling (plastic) and Euler buckling. Once failure was initiated and loading/ strain continued, subsequent failure modes were observed leading to catastrophic failure. For the thick samples $(\mathrm{t} / 1>0.07)$, failure always was observed to be initiated by microbuckling failure, Figure $61(\mathrm{~b})$ and (c). Failure of the slender struts $(\mathrm{t} / 1 \leq 0.07)$ was initiated by Euler buckling. Failure was never observed to be initiated by interplay delamination. Samples that initially failed by plastic microbuckling where observed to transition to a catastrophic fractured kink failure (both single and double fractured kinking was observed) or a shear failure along the formed microbuckle inclination angle. Slender struts initially failing by Euler buckling transitioned to compressive matrix failure in the concave side of the strut leading to large localized strain on the convex side breaking individual fibers in tension and reaching catastrophic failure. 

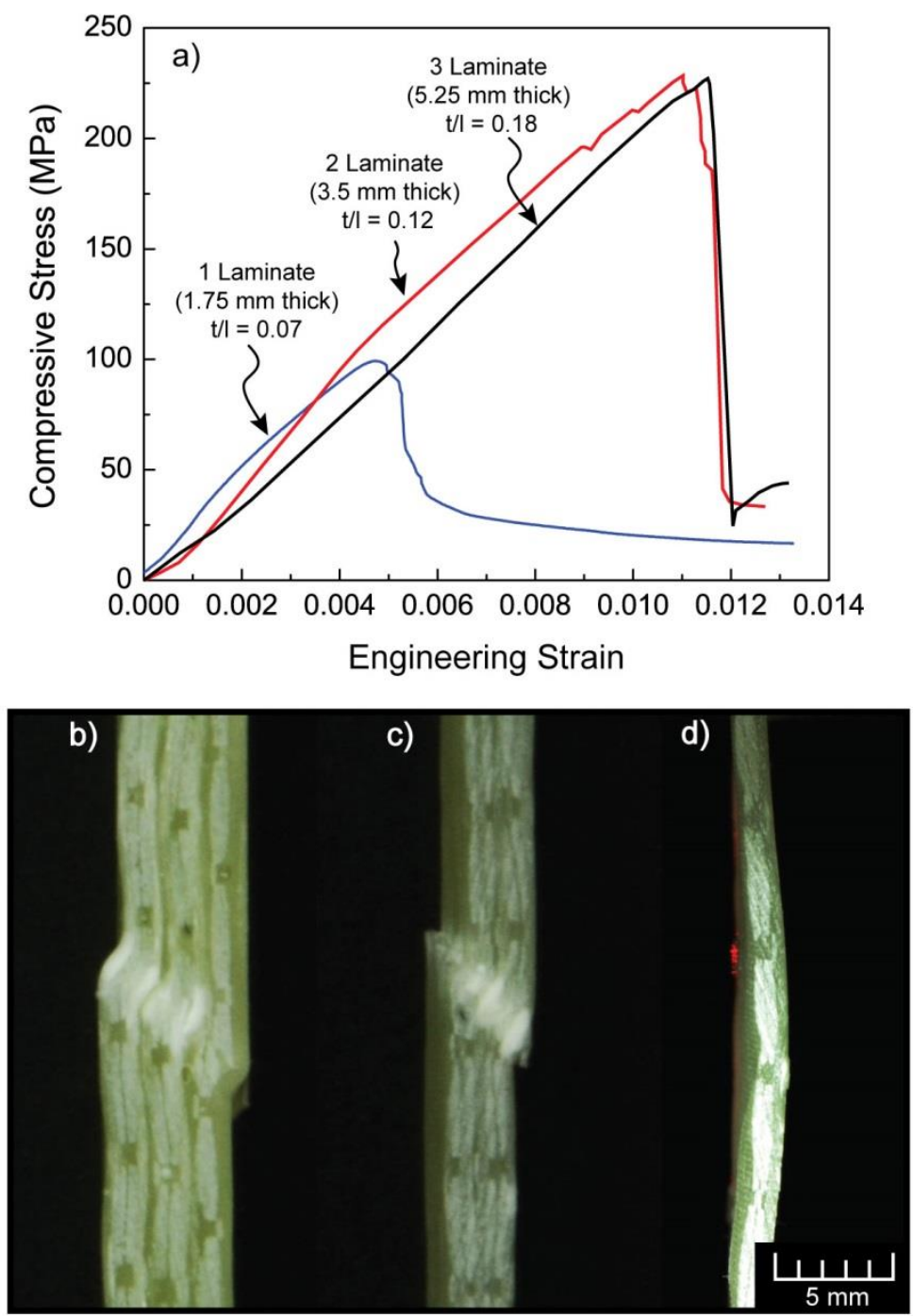

Figure 61: (a) Compressive stress-strain response of the E-glass struts. (b) and (c) are examples of compressive fiber microbuckling and (d) Euler elastic buckling failure of $\mathrm{E}$ glass struts loaded parallel to the weft tow direction with thickness to length ratios of (b) 0.18 (5.25 mm), (c) $0.12(3.5 \mathrm{~mm})$, and (d) 0.07 (1.75 mm).

The elastic modulus of the E and S2-glass composite struts loaded in compression parallel to the weft direction is plotted against fiber volume fraction in Figure 62. The modulus for E-glass composite samples varied from 15 to $23 \mathrm{GPa}$, as the fiber volume fraction was increased from 35 to $60 \%$ while the S2-glass composite samples modulus varied from 12 to 25 $\mathrm{GPa}$ for fiber fractions ranging from 37 to $57 \%$. The S2-glass fiber composite compressive 
modulus in the weft fiber direction is practically indistinguishable from the E-glass counterpart. For comparison purposes, predictions for the compressive modulus are plotted in Figure 62. The predictions are discussed in Section 5.3.3 along with the effect that material properties have on compressive behavior and why the E and S2-glass materials are showing similar compressive responses.

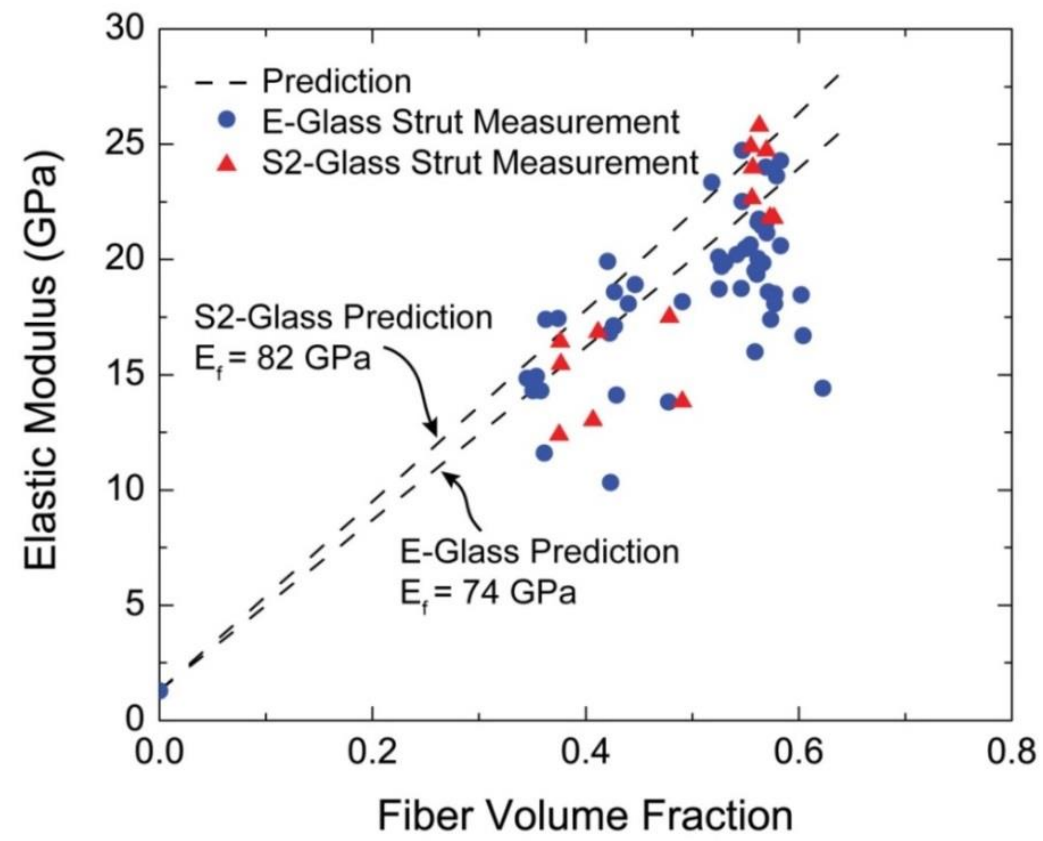

Figure 62: Measured and predicted Young's modulus verses fiber volume for E and S2glass composite struts tested in compression parallel to the weft fiber tows. Fiber waviness has been shown by Cox et al. [109] to reduce the elastic modulus by up to $40 \%$ in 3D woven composites. It is hypothesized that misalignment is the predominant factor for the data scatter of this experiment.

The E and S2-glass composite compressive strength in the weft fiber direction is plotted versus the struts thickness to length ratio (t/l) for samples with fixed $v_{f}=56 \%$ in Figure 63. For this fiber volume fraction, the stubby struts were always observed to fail by fiber microbuckling, Figure 61(b) and (c) while the most slender strut, failed by elastic Euler buckling, Figure 61(d). The transition from Euler to plastic fiber microbuckling was observed at a thickness to length ratio, $t / l$, slightly above 0.07 . Above this value the thickness to length ratio shows no effect on 
failure, with both E and S2 glass samples failing by means of microbuckling. The E-glass composite microbuckling average failure strength was $225 \mathrm{MPa}$ and was almost identical to that of the S2-glass composite (222 MPa) for samples with $v_{\mathrm{f}} \approx 56 \%$. For comparison purposes, predictions for the compressive strength as a function of the thickness to length (t/l) ratio and fiber fraction (for samples with $\mathrm{t} / \mathrm{l}>0.07$ ) are plotted in Figure 63 and Figure 64 . The predictions are discussed in Section 5.3.3

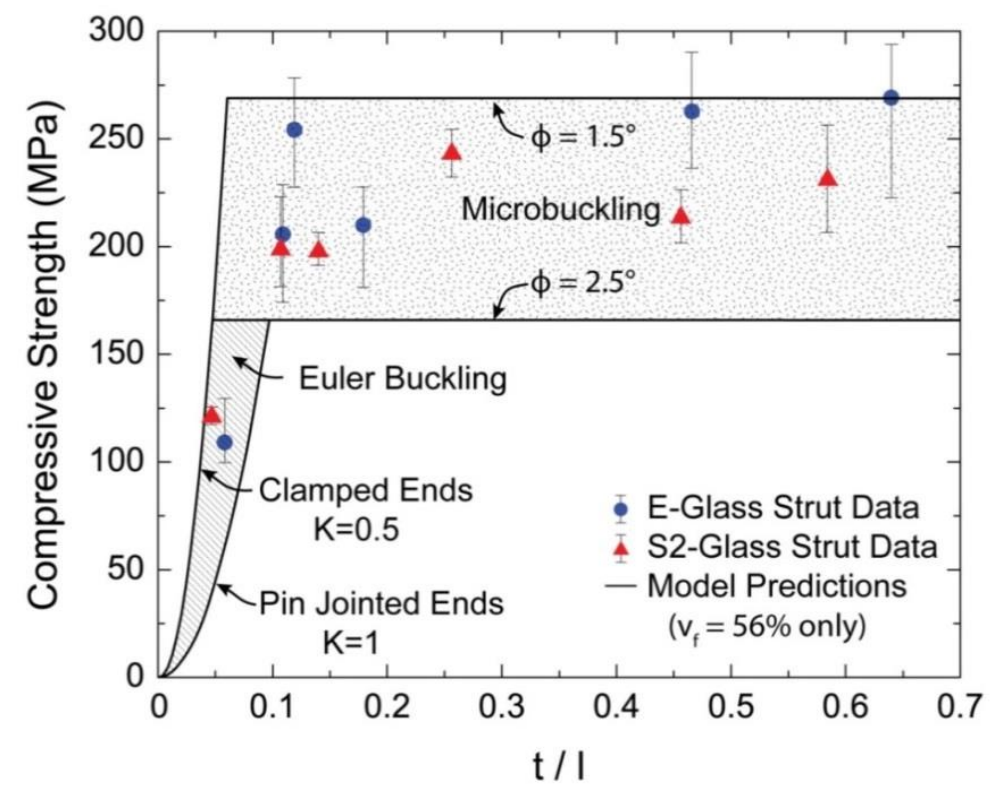

Figure 63: Measured and predicted compressive strength for $E$ and $S 2$ glass composite struts loaded parallel to the weft fiber tows. At low ratios of $t / l<0.07$ the struts failed by Euler (elastic) buckling. As t/l increased, strut failure occurred by fiber microbuckling.

The failure strengths of the $\mathrm{S} 2$ and $\mathrm{E}$ glass fiber composite struts that failed by microbuckling are plotted against fiber volume fraction in Figure 64. For both fiber types, the compressive strength is strongly dependent on the fiber fraction but relatively independent of fiber type. The compressive strength for E-glass varied from 90 to $300 \mathrm{MPa}$, as the fiber volume fraction was increased from 35 to $60 \%$ while the S2-glass varied from 120 to $220 \mathrm{MPa}$ for fiber fractions ranging from 37 to $57 \%$. 


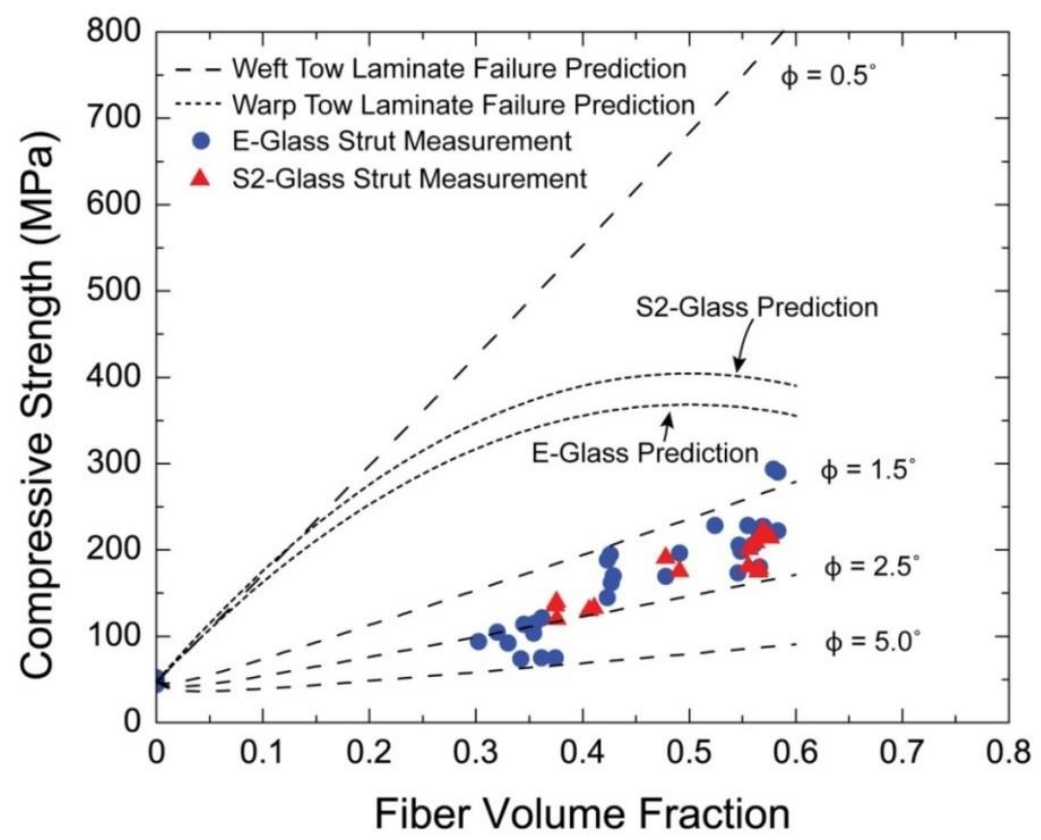

Figure 64: Dependence of compressive strength upon fiber volume fraction for $\mathbf{E}$ and $\mathbf{S 2}$ glass composite struts with $t / l>0.07$ loaded parallel to the weft fiber tows. Failure of weft tow laminates is dependent upon the fiber misalignment angle, $\phi$, but independent of the fiber strength, while failure of the warp tow laminates is dependent upon the fiber strength but independent of the fiber misalignment angle.

None of the 3D woven E or S2-glass fiber composite struts failed by interply delamination. Instead, failure occurred by either elastic buckling $(t / 1=0.07)$ or fiber microbuckling. When failure occurred by elastic buckling, the structure was observed to reach a maximum strength followed by lateral displacement associated with macro-buckling reduced load capability. If the axial displacement was reversed at this stage, the structure returned to its original length during unloading. However, when the strain was increased significantly beyond the critical stress, localized fiber kinking and matrix cracking occurred as secondary failure mechanisms. For samples that failed by fiber microbuckling, permanent damage to the sample occurred at the critical load.

A polished optical micrograph of a 2 laminate E-glass strut $\left(v_{\mathrm{f}}=55 \%, \mathrm{t} / \mathrm{l}=0.111,\right)$ removed from testing at its critical failure stress, $\sigma_{\text {crit }}=198 \mathrm{MPa}$, is shown in Figure 65 . The 
plastic microbuckling is initiated by fiber kinking. A double kink band is observed to form in one of the weft tows at $35^{\circ}$ to the loading direction, Figure $65(\mathrm{a})$. At higher magnification, Figure 65(b), fractured weft fiber kinking can be observed. This is often followed by shear failure along the zone of the fractured kink band and displacement of the fibers in the warp tow. Kink band formation under compression is consistent with other studies on 3D woven composites [114, 119-121].
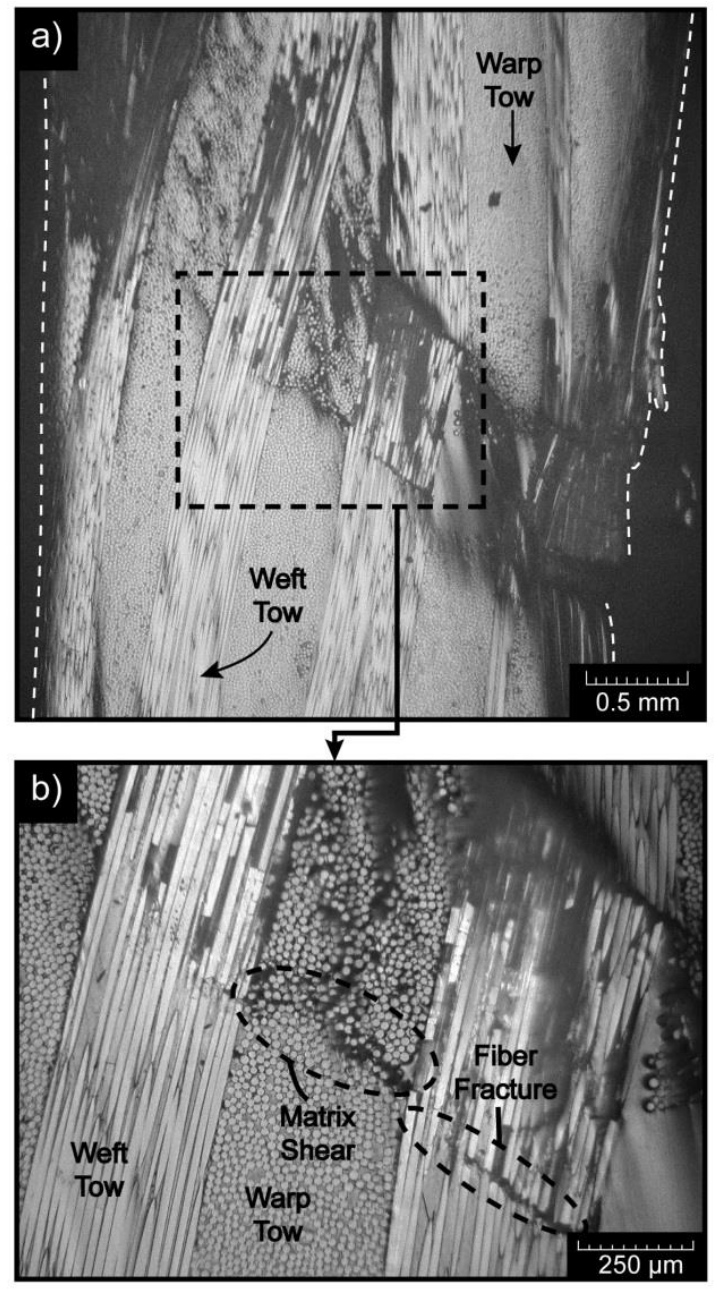

Figure 65: (a) Optical micrographs of an E-glass strut that failed by microbuckling under compressive load. (b) Fiber fracture in weft tows and matrix shear in warp tows accompany the double kink microbuckling mechanism. 


\subsubsection{Analytical Predictions - Strut Compression}

In compression, the modulus of a 3D woven composite is expected to be lower than a laminated unidirectional fiber composite due to the fiber waviness [109] imparted from the zyarn. The compressive modulus of the weft laminates in a 3D woven composite loaded parallel to the weft fiber direction can again be approximated by a rule-of-mixtures expression, Equation (6.2). Likewise, the modulus of the transversely loaded warp tow laminates can be predicted using a constant stress model [25] prediction, Equation (6.3), where the stress in both the fiber and matrix is assumed approximately equivalent, thereby the overall elastic modulus of the composite in compression would be given by Equation (6.1) with an unknown knockdown factor related to fiber waviness. Using the data presented in together with the measured fiber and matrix elastic modulus, the measured modulus values are in moderate agreement with the predictions, Figure 62, with an empirical knockdown parameter up to $40 \%$, consistent with the observations of Cox et al. [109].

In compression, the failure mechanism is not material yielding (as in tension), but rather buckling. Thin struts are observed to fail initially by elastic (Euler) buckling, a typical geometrically driven failure mode. Struts tested with a low aspect ratio $(t / 1=0.7)$ have strengths consistent with the Euler buckling mode failure prediction.

$$
\sigma_{\text {Euler }}=\frac{\pi^{2} E_{c}}{12 K}\left(\frac{t}{l}\right)^{2}
$$

where $K$ is a clamping condition dependent coefficient ( $K=1 / 2$ for fully clamped ends, $\mathrm{K}=1$ for pin jointed ends) and $E_{c}$ is the elastic modulus of the composite strut. As the thickness to length ratio is increased, the failure transitions from elastic buckling to plastic microbuckling. In the 
microbuckling regime, the strut geometry at a fixed fiber volume fraction has no significant effect upon the compressive strength, as plotted in Figure 63. To predict the plastic microbuckling strength, the 3D woven architecture is approximated by a multi-laminate system shown in Figure 66 with the weft fiber tows in the (axial) loading direction and the warp fiber tows are in the transverse loading direction. An iso-stress approximation is applied to the warp fiber tows and the critical failure strength of the warp fiber laminates is simply taken to be equivalent to the epoxy compressive strength.

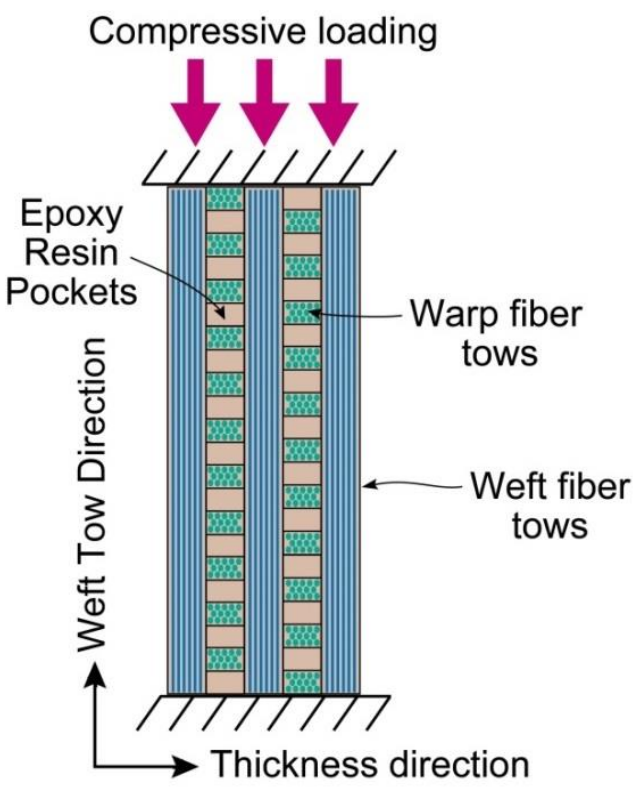

Figure 66: Illustration of the iso-strain loading diagram for the micromechanical model of a single laminate 3D woven strut. The micromechanical model assumes including equal tow spacing, equal tow sizes, and the absence of the z-yarn.

Argon's predicted plastic microbuckling fiber failure stress [118] combines the matrix shear strength and initial fiber misalignment to predict the unidirectional composite failure strength;

$$
\sigma_{f}=\frac{\tau_{m}}{\bar{\phi}}
$$


where $\phi$ is the initial (average) fiber misalignment angle (in radians) and $\tau_{m}$ is the matrix shear strength. Fleck [119] argues that a rule-of-mixtures approach can be utilized with Argon's unidirectional composite microbuckling prediction to predict the fiber volume fraction dependent compressive strength of a composite. Assuming the weft tow laminates fail by fiber microbuckling, the weft laminate critical microbuckling strength would be;

$$
\sigma_{\text {weft-critical }}=\sigma_{f} v_{f}+\sigma_{m}\left(1-v_{f}\right)
$$

where the weft fiber volume fraction is given by $v_{\mathrm{f}}$ and $\sigma_{\mathrm{m}}$ is the compressive strength of the matrix.

An iso-strain analysis of the model composite, Figure 66, can then be used to determine the effective compressive strength of the 3D woven composite. Since the compressed warp and weft fiber laminates will be elastically strained an identical amount, the displacements in the warp and weft laminates $\left(\delta_{\text {weft }}\right.$ and $\left.\delta_{\text {warp }}\right)$ will be equal and given by Hooke's law;

$$
\delta=\frac{P l}{A E}
$$

where $\mathrm{P}$ is the applied force, $\mathrm{A}$ the laminate cross sectional area, $\mathrm{L}$ the laminate length and $\mathrm{E}$ the Young's modulus of each laminate. By equating Equation (6.8) for each laminate the force supported by the weft laminates can be found;

$$
P_{\text {weft }}=P_{\text {warp }} \frac{A_{\text {weft }}}{A_{\text {warp }}} \frac{E_{\text {weft }}}{E_{\text {warp }}}
$$

The weft strength can then be written;

$$
\sigma_{\text {weft }}=\sigma_{\text {warp }} \cdot \frac{E_{\text {weft }}}{E_{\text {warp }}}
$$


The critical compressive strength will be determined by failure of either the warp or weft laminates. If failure occurs in the warp laminate, the compressive strength is given by;

$$
\sigma_{33-\text { crit }}=\frac{\sigma_{m} A_{\text {warp }}}{A_{\text {total }}}\left(1+\frac{A_{\text {weft }}}{A_{\text {warp }}} \frac{E_{\text {weft }}}{E_{\text {warp }}}\right)
$$

If failure occurs in the weft laminates first the critical stress is;

$$
\sigma_{33-\text { crit }}=\frac{\sigma_{\text {weft-critical }} A_{\text {weft }}}{A_{\text {total }}}\left(1+\frac{A_{\text {warp }}}{A_{\text {weft }}} \frac{E_{\text {warp }}}{E_{\text {weft }}}\right)
$$

With the assumption that the overall fiber volume fraction is equivalent in both the warp and weft laminates and assuming no porosity within the strut, the area fraction will be equal to the weft and warp fiber fractions, $f_{\text {weft }}$ and $f_{\text {warp }}$, within the 3D weave. The directional fiber fractions are given by;

$$
f_{\text {weft }}=\frac{A_{\text {weft }}}{A_{\text {total }}}
$$

and

$$
f_{\text {warp }}=\frac{A_{\text {warp }}}{A_{\text {total }}}
$$

If we ignore the presence of the z-yarn, the warp and weft directional fiber fractions are approximately $50 \%$ for both E and S2 glass, Equations (6.11) and (6.12) can be rewritten to give;

$$
\sigma_{33-\text { crit }}=\sigma_{m} f_{\text {warp }}\left(1+\frac{f_{\text {weft }}}{f_{\text {warp }}} \frac{E_{f} v_{f}+E_{m}\left(1-v_{f}\right)}{\left(\frac{v_{f}}{E_{f}}+\frac{\left(1-v_{f}\right)}{E_{m}}\right)^{-1}}\right)
$$

and 


$$
\sigma_{33-\text { crit }}=f_{\text {weft }}\left(\sigma_{f} v_{f}+\sigma_{m}\left(1-v_{f}\right)\right)\left(1+\frac{f_{\text {warp }}}{f_{\text {weft }}} \frac{\left(\frac{v_{f}}{E_{f}}+\frac{\left(1-v_{f}\right)}{E_{m}}\right)^{-1} v_{f}+E_{m}\left(1-v_{f}\right)}{v^{-1}}\right)
$$

Equation (6.15) predicts critical failure of the strut when initiated by failure in the warp tow laminates while Equation (6.16) predict the overall critical failure of the strut when ply failure is initiated by failure in the weft tow laminates and is plotted in Figure 64 .

These strength predictions for weft and warp tow initiated failure, Figure 64, are plotted for initial average fiber misalignment angles ranging from $0.5^{\circ}$ to $5^{\circ}$. The model shows two possible methods of failure initiation, warp or weft tow laminate failure. Failure of the weft tow laminates is dependent upon the fiber misalignment angle, but independent of the fiber strength. Conversely, failure of the warp tow laminates is dependent on the fiber strength but independent of the fiber misalignment angle. Failure can be initiated by limiting strength in either or both laminates simultaneously. The model predicts that with an initial fiber misalignment angle of $1.5^{\circ}$ and greater, failure will be initiated in the weft tow laminates. However, as the misalignment angle is reduced, failure transitions to an initiation by the warp tow laminates. If the overall initial average fiber misalignment can be reduced to $0.5^{\circ}$, the model predicts that effectively at all fiber volume fractions, failure either initiates with the warp tow laminates or occurs simultaneously with the weft tow laminate failure. Comparison with experimental data indicates that the average initial fiber misalignment angle ranges from $1.5^{\circ}$ to $2.5^{\circ}$ and always initiates in the weft tow laminates. This predicted misalignment is in agreement with measured misalignment from XCT images. 
Struts with aspect ratios sufficiently low avoid elastic buckling and fail by plastic microbuckling. Microbuckling is predicted to be dependent upon the misalignment angle, matrix shear strength, and fiber volume fraction. Using optical microscopy and XCT images the misalignment angles of the weft (axially loaded) tows illustrate tows with large misalignments $\left(11.2^{\circ}\right.$ and $22.4^{\circ}$ for E and S2-glass, respectively). However, these extremes do not appear to govern the overall strength. Based on the developed micromechanical model, which combines an initial average fiber misalignment angle and matrix shear strength into a modified form of Argon's plastic microbuckling expression, Equation (6.7), a prediction of the critical compressive load for axial weft tow laminates loading as a function of fiber fraction is developed. This model predicts that the samples that fail by microbuckling will have an initial average fiber misalignment angle approximately ranging from $1.5^{\circ}$ to $2.5^{\circ}$ degrees. Measurements show that for the E and S2-glass struts, the axially loaded weft tows yield average tow misalignments of $1.5^{\circ}$ and $1.6^{\circ}$ degrees, respectively, for the positions exhibiting greatest waviness (logically determined to be the likely position of failure). The conclusion is that the average of all tow misalignments provides a representative value of overall initial average fiber misalignment and good agreement exists between the model and observations.

The elastic buckling strength of slender struts $(t / 1 \leq 0.07)$ was governed by the struts aspect ratio and the fiber volume fraction and type (which influences the composite strut modulus). However, during plastic microbuckling, the fiber misalignment angle, the shear strength of the epoxy matrix, and the fiber volume fraction govern the composite strength. The strut compressive strength was measured and predicted to be relatively insensitive to the tensile strength of the fibers. At high fiber fractions, the microbuckling strengths were $225 \mathrm{MPa}$ for both E and S2-glass composite struts. 
At high fiber fractions $\left(55 \%<v_{\mathrm{f}}<60 \%\right)$, the tensile strength of E-glass composite was $\approx$ $450 \mathrm{MPa}$ while that of the S2-glass fiber composite (made with stronger fibers) was closer to 650 $\mathrm{MPa}$. The tensile strength in both fiber systems was 2-3 times that measured in compression because of the high fiber misalignment angles present in the $3 \mathrm{D}$ woven composites. While the use of z-yarns was successful in increasing resistance to delamination, this was achieved at the cost of significant fiber misalignment, and reduced compressive strength.

While there is a clear strength advantage to using higher strength fibers for tensile loading where failure is governed by fiber fracture, the fiber strength is much less important under compression loading where microbuckling dominates the response. In practical compressive applications, inexpensive E-glass fibers could be substituted for high strength fibers (S2-glass) to reduce cost of a structure while maintaining similar structural properties. 


\section{Chapter 7}

\section{Hybrid Corrugated Core Sandwich Panel}

\section{Quasi-Static Compression}

The hybrid foam glass epoxy corrugated core cellular structure sandwich panel was created by substituting a corrugated glass fiber cellular structure into a PVC foam sandwich panel developed by Latourte et al. [11]. The goal is to discover the failure mechanisms associated with this structure, measure the material properties (including stiffness, strength, densification strain, and energy absorption), and create models to predict the material properties of this structure. Further, a comparison of the structures response to the classic bendingdominated foam core models is presented for comparison purposes. This core topology provides a unique structure that has not previously been investigated. This study is solely focused on the rear-face supported compressive behavior. The quasi-static and dynamic compressive response will provide useful insight to future designs of similar structures or materials.

\subsection{Single-Tier Sandwich Panel}

\subsubsection{Test Methodology - Single-Tier}

Single tier hybrid sandwich panels are investigated under quasi-static compression with a fully supported rear-face boundary condition. Samples were constructed to a width of three unit 
cells to provide a representative sample of the core. Four different relative densities of the structure was manufactured by varying the strut slenderness ratio (t/l) and used either 1,2 , or 3 laminate thick struts for testing. Variation in the number of laminations per web allowed the slenderness ratio (t/l) to be varied from 0.07 to 0.25 which provided a relative density (specifically an open core relative density) to be varied from 15 to $40 \%$. All specimens were tested at an ambient temperature of $25^{\circ} \mathrm{C}$ and a strain rate of $10^{-3} \mathrm{~s}^{-1}$. Measurements were made using a screw-driven Instron universal mechanical test machine utilizing a $300 \mathrm{kN}$ load cell for nominal stress measurements and a laser extensometer to measure the core strain between the two facesheets. The tests used a lateral confinement jig to inhibit lateral shear failure of the core/facesheet nodal attachment commonly seen in corrugated structures. Unloading-reloading curves (not shown) were used to determine the modulus.

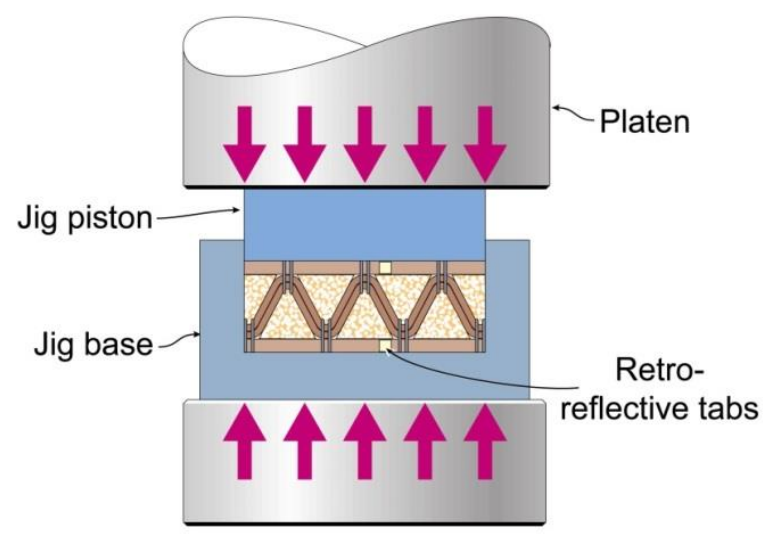

Figure 67: The compression fixture for the hybrid sandwich panel provides rear face and lateral boundary condition support during testing.

\subsubsection{Experimental Results - Single-Tier}

A minimum of five samples was tested for each structure density being evaluated to determine the strength, modulus, densification strain, and energy absorption for each structure. The compressive stress-strain responses of the four core designs (reported as a function of the 
calculated open core relative densities) is shown in Figure 68 for representative samples of the multi-cell (three cells wide) foam-filled and empty (open) core structures. During initial loading, all samples deform in a linear elastic manner, reaching a peak yield strength at strain levels less than $12 \%$. The critical strength is reached, failure occurs by either Euler buckling or plastic micro-buckling of the struts, and inelastic collapse ensues.

The response of the samples shows a plateau strength, but at a significantly reduced level than the observed peak strength (50-80\% of the initial peak strength). This strength level is observed to be approximately maintained for the foam-filled cores and gradually diminished for the open core structures until a densification limit approaches. At the onset of densification, material consolidation changes the approximately flat stress-strain response to rapid strain hardening. Increases in the relative density of the structure increase the observed peak strength and show a decrease in the densification strain. Samples with foam-filled cores showed an increase in energy absorption over the open core samples.
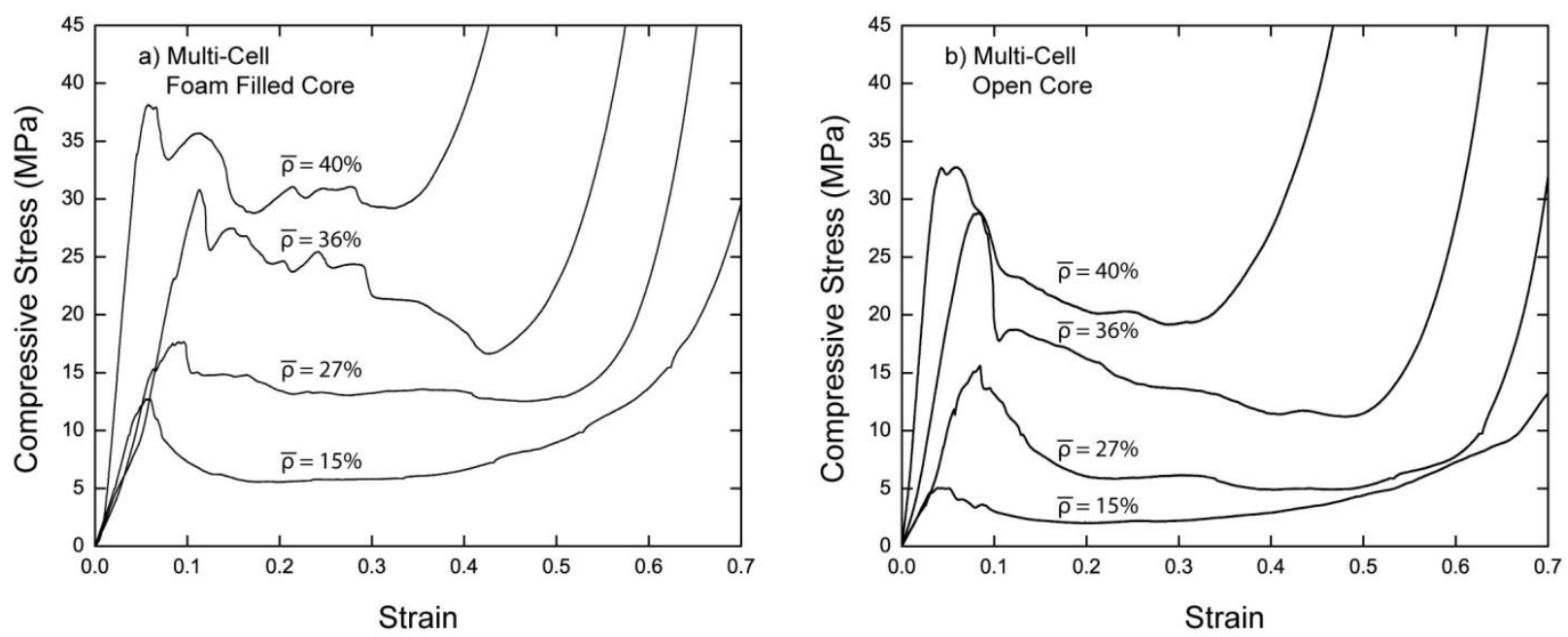

Figure 68: Compressive stress-strain response for the multi-cell glass-epoxy corrugated sandwich structures for the a) Divinycell H130 foam-filled structure and b) open core structure. 
Imperfections in the manufacturing process also led to the testing of single, unit cell structures. It was observed that during the initial loading of the structure, slight variations in nodal height resulted in unequal loading at the beginning of the test and leading to rather large observed compressive strains. To limit the effect of the imperfections, unit cell samples were manufactured with a layer of epoxy placed on both the top and bottom facesheets. The epoxy filled uneven areas of the sample and leveled the surfaces to create parallel loading planes. The stress-strain response for the imperfection-compensated, single unit-cell structures is shown in Figure 69.
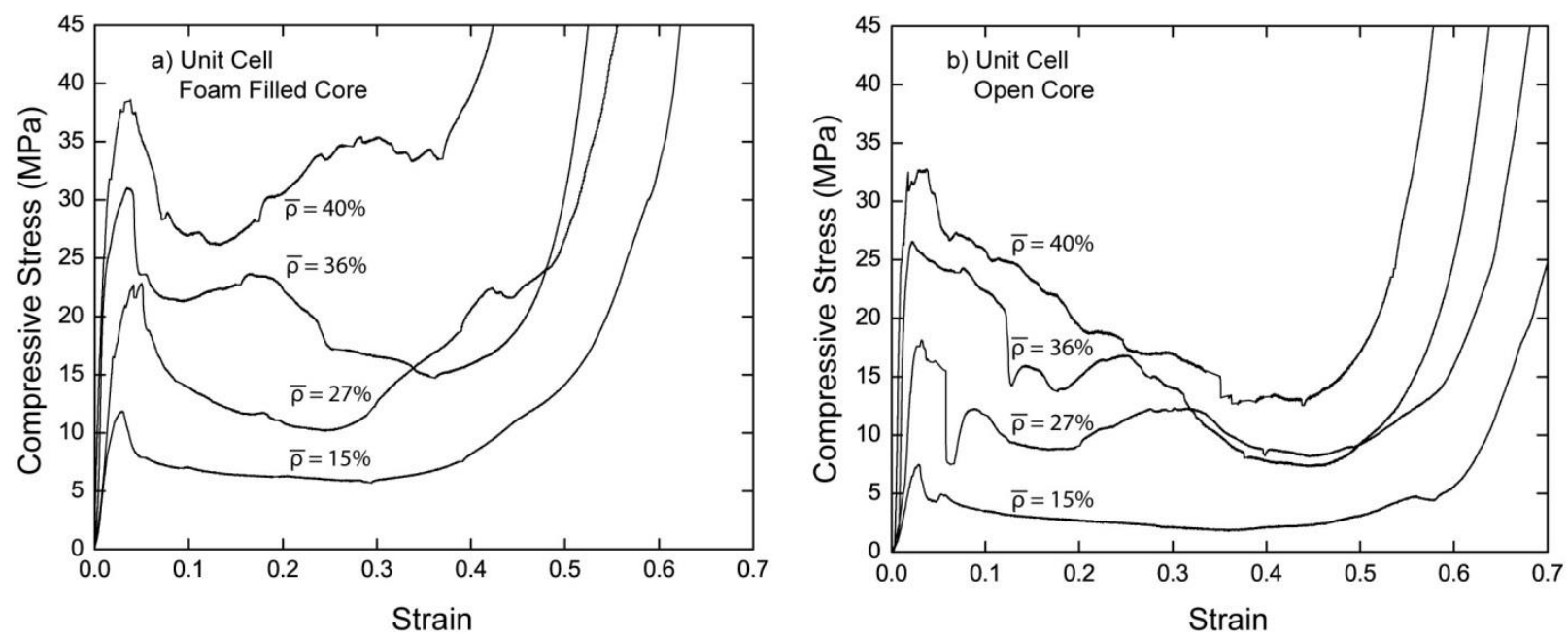

Figure 69: Compressive stress-strain response for unit-cell glass-epoxy corrugated sandwich panel structures for the a) Divinycell H130 foam-filled structure and b) open core structure.

The compressive stress-strain responses of the single, unit-cell core design exhibits a similar stress-strain response to their multi-cell counterparts with one primary difference. The single, unit cell cores show an increase in stiffness throughout the linear elastic loading phase, while the yield strengths and densification strains remain nearly identical to the multi-cell experiments (for both foam-filled and open unit cell core structures). The difference is attributed to the imperfections in the structure, as previously discussed in Section 5.5. The "as- 
manufactured" multi-cell structures show front facesheet waviness and height offsets that result in non-equally distributed loading over the structure. As a result, a single unit cell within the multi-cell structure can support an unequal percentage of an applied load and alter the strain response. Further, as out-of-plane compressive loading on the wavy facesheets occurs, the facesheets will flatten/straighten and account for a small, yet not insignificant, amount of the total strain on the structure. This effect will lower the overall through thickness elastic modulus of the panel. By evaluating a single unit cell and further processing the facesheets to become both flat and parallel, a more ideal loading scenario is created to provide equal load distribution on the core struts and remove measurable strain from through thickness compression of the facesheet. With improved manufacturing methods, the multi-cell structures could be built to the same specifications of the unit cell structure to increase the through thickness elastic modulus.

Photographs taken during the testing of core Design $3(t / l=0.14)$ are shown for the foam-filled and open core structures in Figure 70 and Figure 71, respectively. The foam-filled and open core structures were compressed to a strain of 50\% with the associated core response captured. Initial failure for both structures was due to strut microbuckling where the peak failure strength occurred at a strain between 6 to $8 \%$ for the multi-cell samples and between 3 to $5 \%$ in the single, unit cell sample. Variability in strut length and inclination angle, creating nonparallel facesheets and unequal loading, contributing to the reduced modulus observed in the multi-cell samples. The rise in stress at the densification limit is observed as the strut buckles and impinges upon itself, giving rise to the load bearing ability once again. 


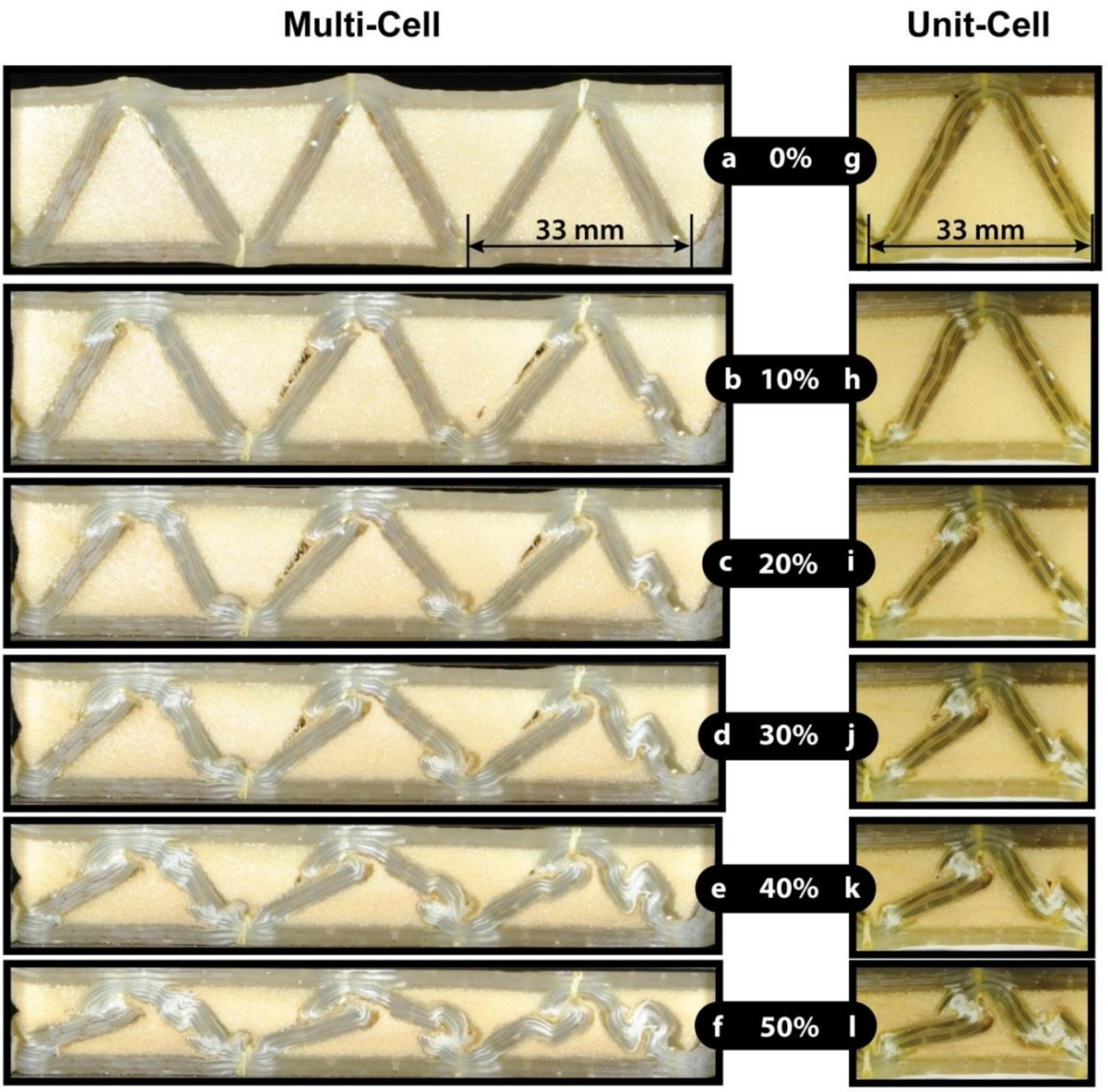

Figure 70: Photographs taken during compression of the foam-filled "Design 3" sandwich structure. Initial failure of the trusses occurs by micro-buckling of the struts. Parts a-f correspond to the multi-core structure and parts g-l correspond to the single, unit-cell structure for strains of $0 \%, 10 \%, 20 \%, 30 \%, 40 \%$, and $50 \%$ respectively. The "white" regions correspond to matrix crazing. 


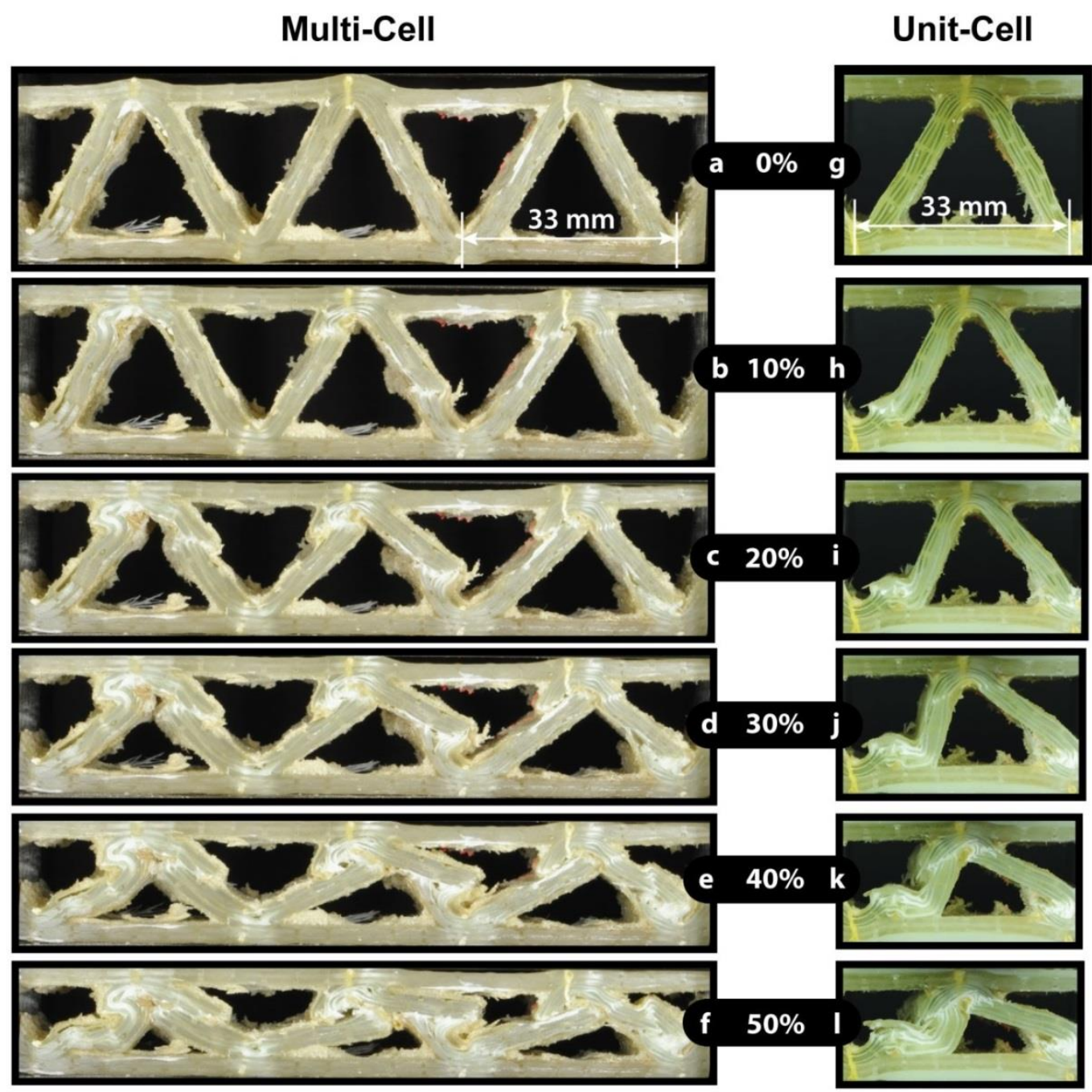

Figure 71: Photographs taken during compression of the open core "Design 3" structure. Initial failure of the trusses occurred by micro-buckling of the struts. Parts a-f correspond to the multi-core structure and parts g-l correspond to the single, unit-cell structure for strains of $0 \%, 10 \%, 20 \%, 30 \%, 40 \%$, and $50 \%$ respectively. The "white" regions correspond to matrix crazing.

Throughout all of the compressive testing of the foam-filled and open core corrugated sandwich structures, failure by elastic buckling or localized microbuckling was observed in the struts. Microbuckling failure occurred by fiber kinking and both unfractured and fractured double kinking was observed. As the loading continued, secondary failures could be observed to include strut shear. As composites often fail catastrophically after yielding, it is sometimes 
difficult to distinguish between fractured double kinking and shear after failure has occurred.

The failure mechanisms of the corrugated composite sandwich panel are illustrated in Figure 72.

(a)

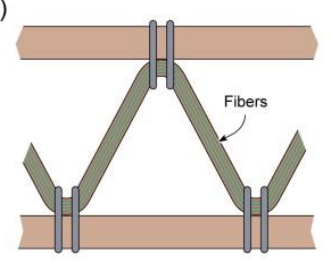

Undeformed
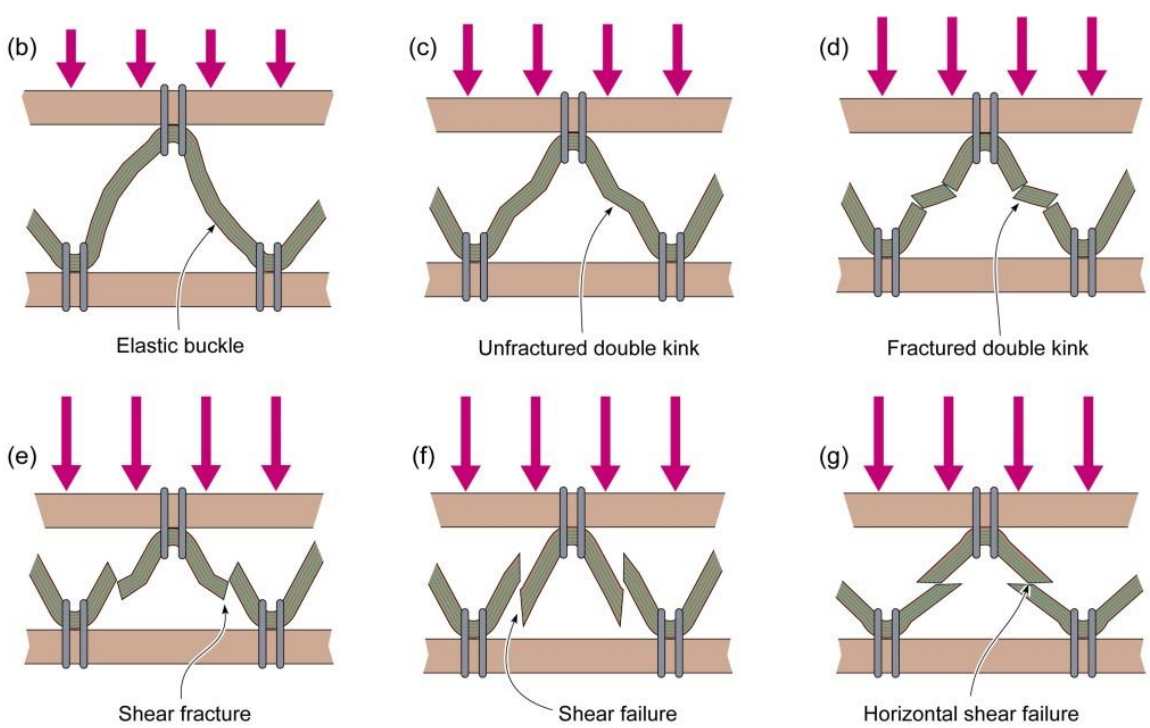

Figure 72: The (a) composite corrugated sandwich structure exhibited many failure modes (b to g) during the compression testing up to the densification limit. Predominant failure was initiated by (b) elastic buckling, (c) unfractured double kinking of the fiber tows or (d) fractured fiber kinking of the fiber tows. Subsequent shear fractures were observed after fiber kinking occurred.

The compressive modulus measured for the hybrid corrugated sandwich structures showed a tremendous difference between the single unit-cell structures and multi-cell structures. As shown in Figure 73, the modulus of the multi-cell cores ranged from 0.2-0.9 GPa, while the unit-cell structures ranged from 0.6-3.9 GPa. The difference in response between the multi-cell and unit cell structures is attributed to both the unequal loading among the core struts and facesheet waviness, as discussed earlier in this chapter. The unloading modulus of the samples 
varied approximately linearly with the relative density of the core. Modeling results are overlaid on Figure 73 for comparison purposes but discussed in section 7.1.3. The scatter in the experimental data is primarily due to the differences in the fiber volume fraction, $v_{\mathrm{f}}$, of the strut. The fiber volume fractions of the core struts ranges from 30 to $40 \%$ and these model predictions are presented in Figure 73. While the compressive modulus of the foam-filled cores was predicted to increase relative to the open core structures by around $1-2 \%$, this effect could not be confirmed from the experiment due to data scatter.

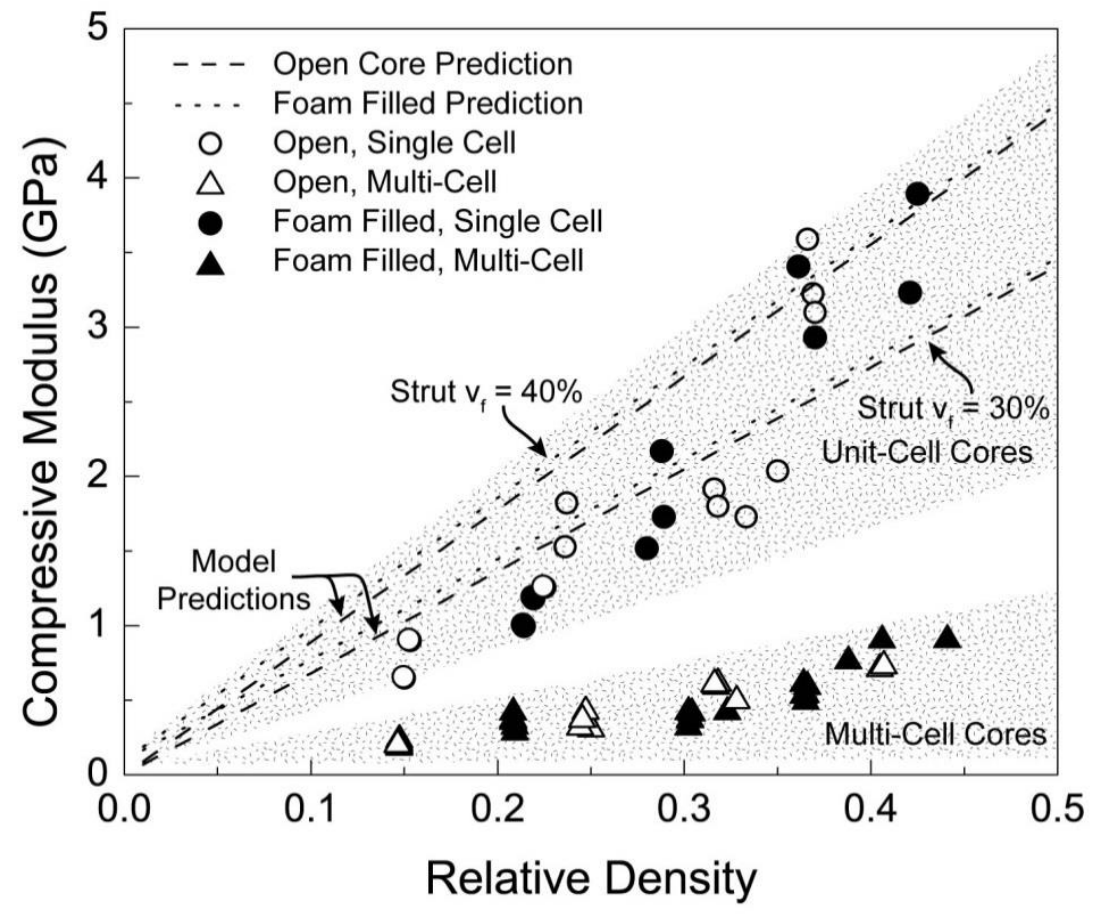

Figure 73: Elastic modulus data and micromechanical predictions for the foam-filled and open core structures as a function of relative density. Imperfections in multi-cell samples resulted in non-uniform strut loading and a measured modulus significantly lower than model predictions. The measured modulus of the single unit-cell samples are in good agreement with the micromechanical model predictions representative of the fiber volume fractions measured in the core struts $\left(30 \%<v_{\mathrm{f}}<40 \%\right)$.

A histogram displays the initial (peak) strength for the foam-filled and open core structures for the relative densities tested and is shown in Figure 72(a) and (b), respectively. The strength rises as a function relative density but greater than strictly a linear dependence. The 
peak strengths for the filled core varied from 12 to $37 \mathrm{MPa}$ as the relative density increased from 15 to $40 \%$. The strength of the open core samples for each corresponding relative density was less, ranging from 5 to $33 \mathrm{MPa}$ over the same relative density range. Comparing the average strengths of the foam-filled and open cores, Figure 74(a) and (b), it is observed that for all structures that fail by plastic microbuckling, a strength increase of $3 \pm 0.7 \mathrm{MPa}$ is observed, the approximate strength of the Divinycell H130 foam, resulting in little strut stabilization from the foam interaction and near zero synergism. However, for the slender, single laminate struts $(\mathrm{t} / \mathrm{l}<$ 0.07) failure occurred by Euler buckling for the open core and transitioned to plastic microbuckling for the foam filled core. An increase of $7 \mathrm{MPa}$ was observed by the strut stabilization from the foam and a synergistic effect is observed. Modeling results are overlaid on Figure 74 for comparison purposes but discussed in section 7.1.3. The experimental range in strength is represented by the error bars, along with the average strength of all cores tested.
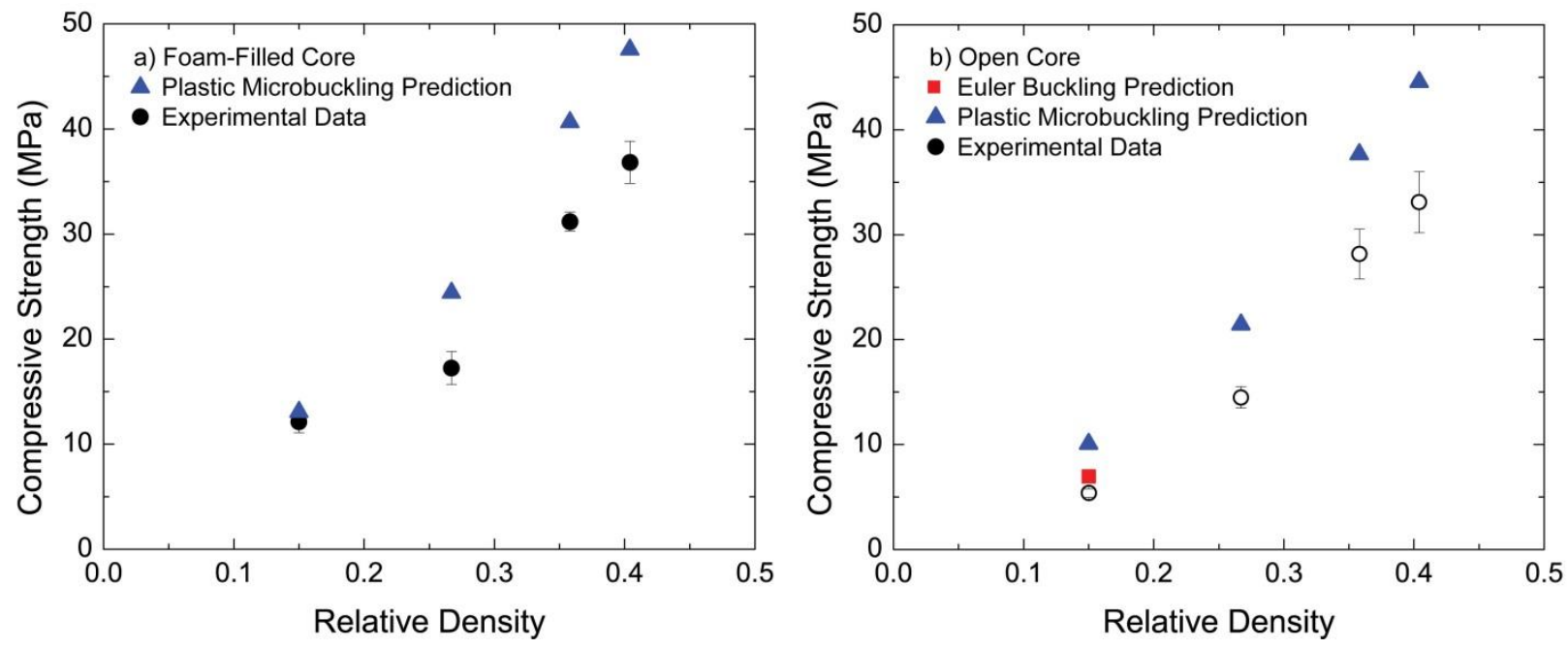

Figure 74: Peak compressive strength measurements and predictions for the corrugated composite core. a) Divinycell H130 foam-filled core and b) the open core. Results are shown as a function of the calculated relative density of the open core structure only. The predicted strengths of the corrugated structures are based on the experimentally measured critical strengths of the strut, Table 8. The range and average measured strength is shown. 
The resulting specific strength and specific modulus for the single laminate unit cell structures is plotted in relation to common metals and several polymers. The hybrid corrugated sandwich panel does provide a higher specific strength to common structural steels, brass, and many polymers. However, the hybrid core does not match the specific strength of aluminum 6061 and stainless steel 304L. The hybrid corrugated core does provide a lower specific modulus than all metal bulk materials.

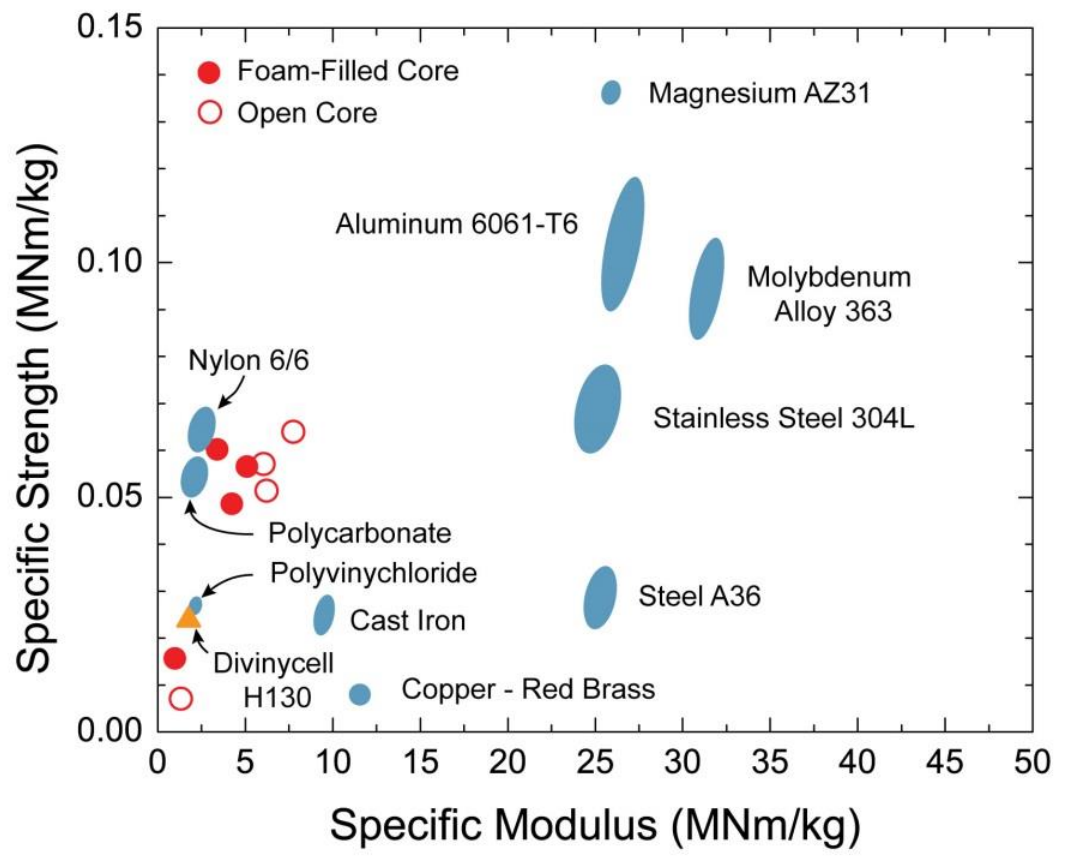

Figure 75: The specific strength versus the specific modulus for the hybrid composite corrugated sandwich panel is compared with several metals and polymers. The hybrid structure provides a higher specific strength than common structural steel and cast iron, but does not match the performance of aluminum 6061 or stainless steel 304L, previously studies for blast applications.

The densification strain of the corrugated sandwich panels are measured from the stressstrain response. The densification strain, $\varepsilon_{D}$, is defined as the strain at which the stress of the compressed structure reaches an equivalent level to the initial peak strength of the structure, as 
illustrated in Figure 24. Increases in energy absorption under a specifically defined stress criterion occur when the densification strain is increased. Densification strain values for the cores in this study were obtained from the stress-strain curves shown in Figure 68 and Figure 69, and are presented in Table 6 and plotted in Figure 76. Data for the densification of H130 Divinycell foam along with other Divinycell foam densities [122] are also shown in Figure 76 along with model predictions discussed in sections 7.1.3 and 7.3. The densification strains of the single and multi-celled corrugated structures depend weakly upon the relative density. The open and foamfilled structures exhibit densification strains of approximately $60 \%$ and $48 \%$ respectively for open cell relative densities in the $15-40 \%$ range. The densification strain relationships for the corrugated cores were markedly different to those of the Divinycell foam.

Table 6: Measured densification strain values of the tested structures based on the relative density of the composite core web.

\begin{tabular}{|c|c|c|c|c|}
\hline \multirow{2}{*}{$\begin{array}{c}\text { Relative } \\
\text { Density }\end{array}$} & \multicolumn{2}{|c|}{ Foam-Filled Structure } & \multicolumn{2}{c|}{ Open Core Structure } \\
\cline { 2 - 5 } & Unit Cell & Multi Cell & Unit Cell & Multi Cell \\
\hline $15 \%$ & 0.46 & 0.59 & 0.62 & 0.54 \\
$25 \%$ & 0.45 & 0.57 & 0.61 & 0.65 \\
$32 \%$ & 0.50 & 0.54 & 0.60 & 0.60 \\
$41 \%$ & 0.40 & 0.40 & 0.56 & 0.43 \\
\hline
\end{tabular}




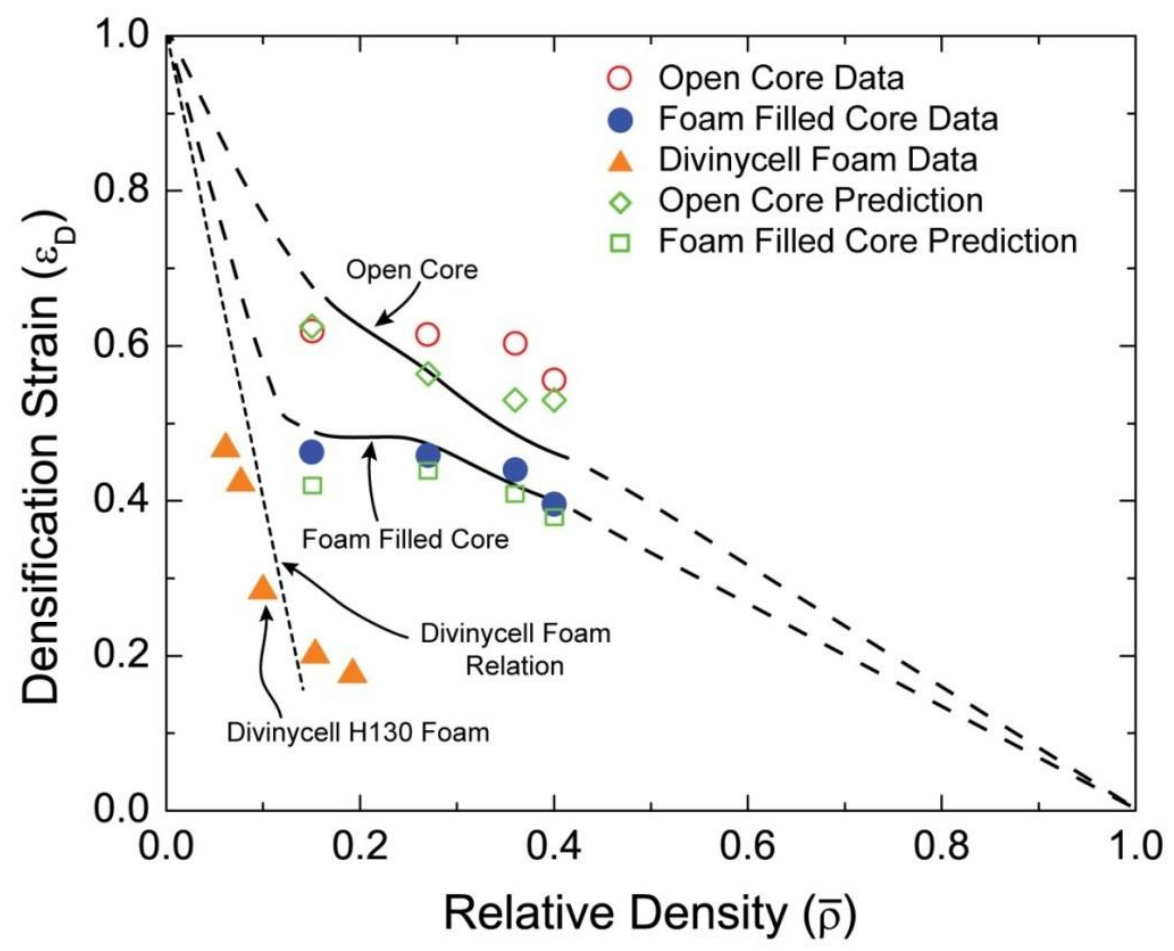

Figure 76: Densification strain measurements are shown for the unit cell structures (open and foam-filled) and various grades of Divinycell foam. The densification strain data for the foams is closely approximated by a linear model proposed by Maiti et al. [58]. Core predictions are based on the developed strut hinge model using specific parameters unique to each core geometry (green markers). The strut hinge densification model is simplified using fixed constants $x=0.70$ and $\omega=6^{\circ}$ (solid black line) with anticipated trends illustrated to the bounding limits (dotted lines).

The energy absorbed per unit volume during the compression of a cellular structure is obtained by integrating the stress-strain response up to the densification strain, $\varepsilon_{\mathrm{D}}$, and is shown for the structures tested here and for several Divinycell foams [122] as shown in Figure 80. The composite cellular structures extend the energy absorption of $\mathrm{H} 130$ foam from $1.1 \mathrm{MJ} / \mathrm{m}^{3}$ to about $13 \mathrm{MJ} / \mathrm{m}^{3}$ while increasing the density from 130 to $800 \mathrm{~kg} / \mathrm{m}^{3}$. A small increase in the average energy absorption is observed between the foam-filled and open core structures, ranging from 1 to $3 \mathrm{MJ} / \mathrm{m}^{3}$. A synergism between the foam and open core is not observed at the lower relative densities, but appears to develop and linearly increase as the as the density increases. 


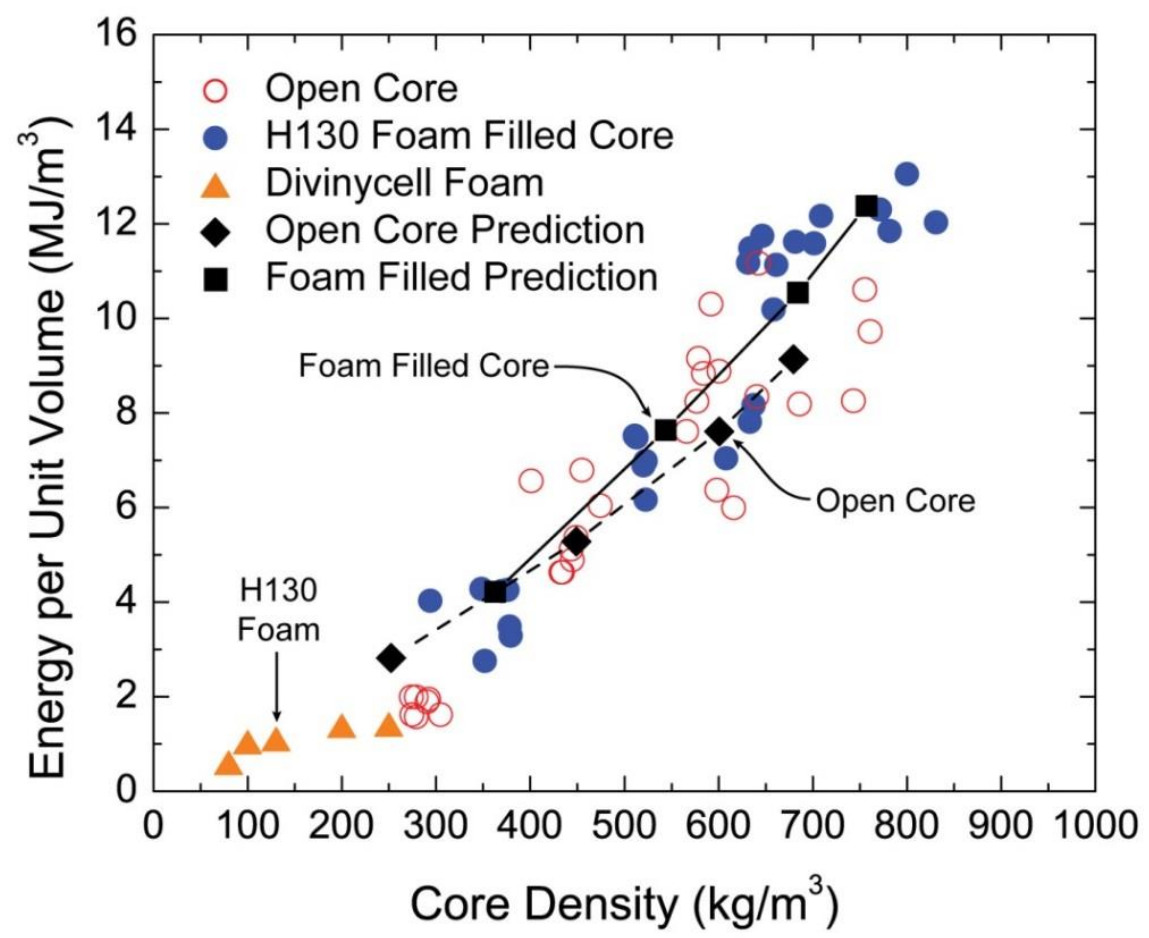

Figure 77: The energy absorbed per unit volume during compression to the densification strain. Data is shown for the empty and foam-filled corrugated core composite structures investigated here and compared to Divinycell foams. Energy absorption predictions (based on the micromechanical models developed in this study) are illustrated for comparison with experimental data.

The energy absorption per unit mass of the corrugated cores is comparable to metal energy absorbing foams, Figure 78. The average energy absorption/ unit mass ranged from 6.4 to $17 \mathrm{~J} / \mathrm{g}$ with a negligible increase in energy absorption with the addition of the foam core for the more dense core structures (higher plateau strength cores) yet a $2 \mathrm{~J} / \mathrm{g}$ energy increase for the lowest density (lowest plateau strength) core structures. No difference in energy absorption was observed between the unit cell and multi-cell cores as anticipated. 


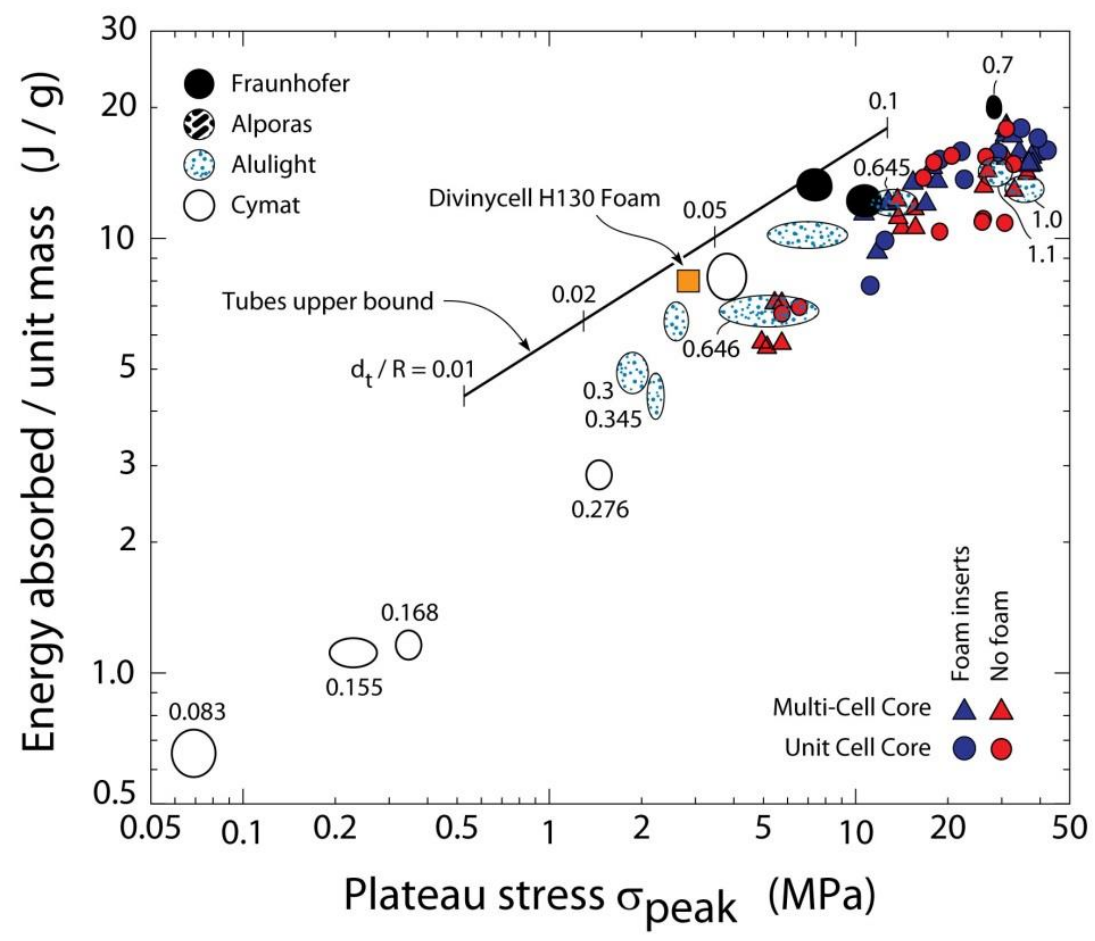

Figure 78: The energy absorbed per unit mass of the composite corrugations and metal foams plotted against plateau stress, $\sigma_{\mathrm{pl}}$. Metal foam data and tube upper bound tube predictions are presented from Ashby et al. [38]. Each foam is labeled with its density in $\mathrm{Mg} / \mathrm{m}^{3}$.

The volumetric energy absorption efficiency, $\zeta$, of a structure can be defined as the ratio of the actual energy absorbed during compression of the structure to its densification strain divided by that of an ideal material, with a flat plateau stress response up to the densification strain.

$$
\varsigma=\frac{U_{\text {actual }}}{U_{\text {ideal }}}
$$

This efficiency parameter, $\zeta$, was determined to be approximately 0.46 for the open core and 0.82 for the foam-filled structure, while the Divinycell foam had a nearly ideal efficiency of 0.99. The lower efficiencies of the corrugated structures were a result of the unstable buckling response of their struts. 
Table 7: Approximate energy absorption efficiencies for the composite sandwich structures and Divinycell H130 foam.

\begin{tabular}{|c|c|}
\hline Structure & $\begin{array}{c}\text { Energy Absorption Efficiency } \\
\zeta(\%)\end{array}$ \\
\hline Foam-Filled Core & 82 \\
Open Core & 46 \\
Divinycell H130 Foam & 99 \\
\hline
\end{tabular}

\subsubsection{Analytical Predictions - Single-Tier}

Predictions for the stiffness, strength, densification strain, and energy absorption are developed through the construction of micromechanical models that reflect the behavior and material properties of the corrugated composite structure. These models are compared with the experimental results to determine the validity of the model prediction.

\section{Elastic Modulus Prediction}

The compressive, elastic modulus of an open corrugated core sandwich panel can be predicted by assuming that all the struts are equal in length and uniformly loaded. Utilizing a free body diagram, Figure 79 , in which the applied load, $\mathrm{F}_{33}$, is oriented parallel to the vertical axis [55], the force in each strut, $\mathrm{F}_{\mathrm{t}}$, can be determined. 
a)

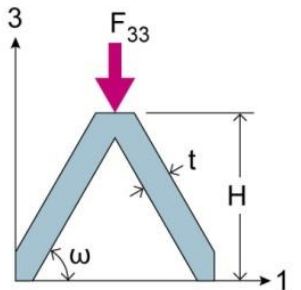

b)

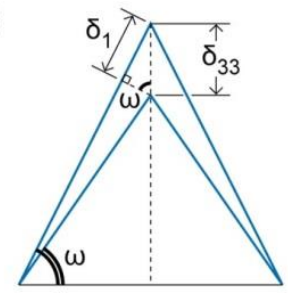

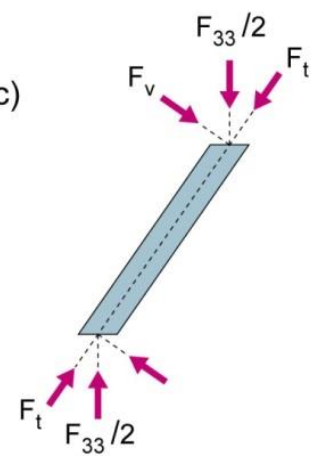

Figure 79: a) Loading diagram for the determination of effective elastic compressive modulus b) illustration of strut deflection assuming small strain and pin jointed approximation and c) free body diagram of strut loading.

Assuming the strain is small, the struts are pin-jointed, the struts are inclined at an angle $\omega$ to the applied force, the core elastic modulus, $\mathrm{E}_{\text {core }}$ depends upon the measured modulus of the strut material, $\mathrm{E}_{\text {strut }}$, and the strut geometry.

$$
E_{\text {core }}=\frac{t E_{\text {strut }} \sin ^{4} \omega}{(t+H \cos \omega-2 c \cos \omega)}
$$

Substituting for the simplified form of the open core relative density (Equation (7.2)), the compressive modulus is predicted to have a linear dependence upon core relative density and strut material modulus.

$$
E_{\text {core }}=\bar{\rho} E_{\text {strut }} \sin ^{4} \omega
$$

To determine the effective modulus of the foam-filled core structures, the rule of mixtures is utilized to combine the open core modulus (Equation (7.3)) with the measured modulus of the foam, $\mathrm{E}_{\text {foam }}$ based upon the assumption that all volume not occupied by struts is filled with foam.

$$
E_{f f-c o r e}=E_{\text {core }}+(1-\bar{\rho}) E_{\text {foam }}
$$


The predicted moduli for the open (Equation (7.3)) and foam-filled/hybrid cores (Equation (7.4)) for measured strut fiber volume fractions, $\mathrm{v}_{\mathrm{f}}$, of 30 to $40 \%$ are plotted on Figure 73 and are in good agreement with the single unit cell measurements. It is worth noting that if the fiber volume fraction of the struts is increased, this enables the strut material modulus to be increased and equally that of the core, critical to the design of a structure requiring an increased stiffness.

\section{Strength Prediction}

The strength of the composite struts exhibit a linear elastic response prior to any method of compressive failure and maintains that response until the critical stress, $\sigma_{c r i t i c a l}$, is achieved when initiated by Euler or plastic fiber micro-buckling. Using a free body diagram, Figure 79, in conjunction with the experimentally measured critical strut strength, Table 8 , the compressive core strength is calculated to be;

$$
\sigma_{\text {core }}=\frac{t \sigma_{\text {critical }} \sin ^{2} \omega}{(t+H \cos \omega-2 c \cos \omega)}
$$

Substituting in the relative density expression of an open core (Equation 4.6), gives a relation between the compressive strength of the core, the core relative density, and the struts compressive strength, given in Equation (7.6).

$$
\sigma_{\text {core }}=\bar{\rho} \sigma_{\text {critical }} \sin ^{2} \omega
$$

The predicted strength for each core, using the strut orientation angles from Table 2 and critical strut strength presented in Table 8, is compared with the experimental data in Figure 74. At the lowest relative density tested, the core failed by Euler buckling and the strut aspect ratio and fiber volume fraction control the strut modulus. At higher relative densities failure of the strut is 
predicted to be by plastic micro-buckling, in agreement with experimental observations. It can be seen from Equation (7.6) that the core strength is predicted to rise approximately linearly with relative density and the critical (buckling) strength of the strut.

Table 8: Replicas of the E-glass struts within the corrugated cores were manufactured and tested to provide "as-manufactured" strut properties for modeling the overall structure properties.

\begin{tabular}{|c|c|c|c|c|c|}
\hline \multirow{2}{*}{$\begin{array}{c}\text { Core } \\
\text { Design }\end{array}$} & \multirow{2}{*}{$\begin{array}{c}\text { Predicted } \\
\text { Open Core }\end{array}$} & \multirow{2}{*}{$\begin{array}{c}\text { Number } \\
\text { Relative Density } \\
(\%)\end{array}$} & \multicolumn{3}{|c|}{ Core Strut Properties } \\
\cline { 4 - 6 } & Laminates & $\begin{array}{c}\mathrm{v}_{\mathrm{f}} \\
(\%)\end{array}$ & $\mathrm{t} / \mathrm{l}$ & $\begin{array}{c}\sigma_{\text {strut }} \\
(\mathrm{MPa})\end{array}$ \\
\hline 1 & 40.4 & 2 & $38 \%$ & 0.248 & 122 \\
\hline 2 & 15.0 & 1 & $33 \%$ & 0.074 & 88 \\
\hline 3 & 26.7 & 2 & $31 \%$ & 0.141 & 92 \\
\hline 4 & 35.8 & 3 & $35 \%$ & 0.212 & 113 \\
\hline
\end{tabular}

The strength of the foam-filled structures can be estimated by using a rule-of-mixtures approach to give a predicted strength.

$$
\sigma_{\text {ff-core }}=\bar{\rho} \sigma_{\text {critical }} \sin ^{2} \omega+(1-\bar{\rho}) \sigma_{\text {foam }}
$$

Figure 74 shows that in most cases the measured foam-filled core strength is well predicted by Equation (7.7). Examination of the experimental strength data for the lowest density open and foam-filled structures reveals that the strength of the foam-filled structure (12 $\mathrm{MPa}$ ) exceeded the sum of the foam strength (3MPa) and the open cell core (5MPa). The strength of struts with a thickness to length ratio (t/l) of 0.07 or less was governed by Euler 
buckling, and it is possible that the foam stabilized the strut by providing lateral pressure to the strut wall during buckling leading to an increase in the critical buckling strength. All structures with $\mathrm{t} / 1>0.07$ failed by microbuckling (at a critical stress much higher than the compressive strength of the foam), and it is unlikely that the foam used here could significantly impede this failure mode.

\section{Densification Strain Prediction}

The densification strain prediction developed by Gibson and Ashby [26] for cellular solid foams does not accurately predict the densification strain of the corrugated sandwich panels. A new model, based on the observed collapse mechanism of the composite strut, is developed to provide a prediction. This model is a unique contribution of this research in the advancement of composite cellular structure mechanical behavior prediction. The densification strain is essential to predict the energy absorption during crushing of the core, and therefore the relationship between densification strain and relative density is also important. It is observed that after initial microbuckling failure occurs during compression, Figure 70 and Figure 71 , the strut develops a hinge that expands laterally along a facesheet. The hinge motion allows the facesheet separation to decrease, the effective strut length (supporting the stress) to decrease, and the strut inclination angle to gradually rise. Eventually, a geometry is reached where the fractional strut length, $x$, that has undergone bending reaches a value of $0.70 \pm 0.03$ of the strut length, 1 . This value is experimentally observed to coincide with the strain at which continued compression occurs at a stress equal to the initial peak in strength. 


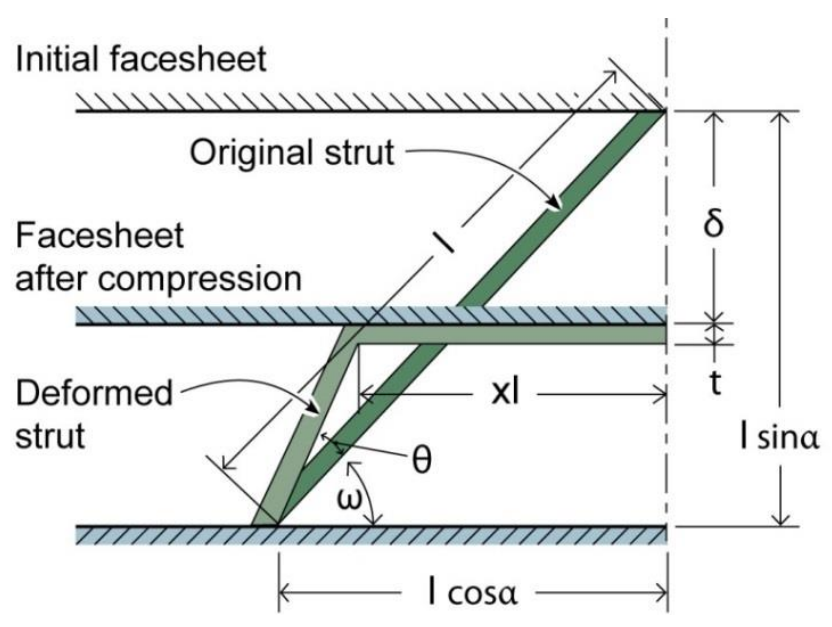

Figure 80: The strut collapse model is developed to predict densification strain of the corrugated sandwich panels. The densification strain is reached when the deformed strut aspect ratio and inclination angle result in cell strengths sufficient to support the structures initial peak strength.

The densification strain of the core can be established from vertical strain-displacement relation;

$$
\varepsilon=\delta / l \sin \omega
$$

where $\delta$ is the out-of-plane unit cell displacement. By summing vertical distances in Figure 80, we see that $\delta+t+l(1-x) \sin (\theta+\omega)=l \sin \omega$ and $\delta=l \sin \omega-t-l(1-x) \sin (\theta+\omega)$. By substituting these expressions into Equation (7.8) we find the compressive strain is given by;

$$
\varepsilon=1-\frac{t}{l \sin \omega}-\frac{(1-x) \sin (\theta+\omega)}{\sin \omega}
$$

By summing the horizontal distances within the cell, it can be shown that $\sin (\theta+\omega)=\sqrt{1-\left(\frac{\cos \omega-x}{1-x}\right)^{2}}$. Substituting this in Equation (7.9) gives an expression for the compressive strain; 


$$
\varepsilon=1-\frac{t}{l \sin \omega}-\frac{1-x}{\sin \omega} \sqrt{1-\left(\frac{\cos \omega-x}{1-x}\right)^{2}}
$$

The densification strain, $\varepsilon_{\mathrm{D}}$, predicted by Equation (7.10) is given by substitution of the measured hinge position, $x$, at densification. Parameters of the core geometry, $t, 1$, and $\omega$ (Table 2) along with the experimental measured hinge position, $x$ (Table 9), provide the densification strain, $\varepsilon_{\mathrm{D}}$, prediction shown in Table 9. The measured and predicted densification strains are compared in Figure 76 and are in good agreement.

Table 9: Predicted densification strain, core strength, and energy absorption from the developed micromechanical models. Average dimensional parameters for each core geometry were used in the prediction. The hinge position (position of strut hinge at time of densification), $x$, was experimentally measured for each core geometry and utilized as an empirical fit in the hinge model.

\begin{tabular}{|c|c|c|c|c|c|c|c|}
\hline & Core type & $\begin{array}{c}\text { Predicted } \\
\text { Open Core } \\
\text { Relative } \\
\text { Density } \\
(\%)\end{array}$ & $\begin{array}{c}\text { Hinge } \\
\text { Position, } \\
\mathrm{x} \\
(\%)\end{array}$ & $\begin{array}{c}\text { Strength, } \\
\sigma \\
(\mathrm{MPa})\end{array}$ & $\begin{array}{c}\text { Densification } \\
\text { Strain } \\
\varepsilon_{\mathrm{D}}\end{array}$ & $\begin{array}{c}\text { Efficiency } \\
\text { Parameter, } \\
\xi \\
(\%)\end{array}$ & $\begin{array}{c}\text { Energy } \\
\text { Absorbed } \\
\left(\mathrm{MJ} / \mathrm{m}^{3}\right)\end{array}$ \\
\hline \multirow{2}{*}{ Design 1} & Open Core & \multirow{2}{*}{40.4} & 72 & 37.4 & 0.53 & 46 & 9.1 \\
\hline & Foam-Filled Core & & 69 & 39.2 & 0.38 & 82 & 12.4 \\
\hline \multirow{2}{*}{ Design 2} & Open Core & \multirow{2}{*}{15.0} & 69 & 9.8 & 0.62 & 46 & 2.8 \\
\hline & Foam- Filled Core & & 66 & 12.4 & 0.42 & 82 & 4.2 \\
\hline \multirow{2}{*}{ Design 3} & Open Core & \multirow{2}{*}{26.7} & 70 & 18.9 & 0.61 & 46 & 5.3 \\
\hline & Foam- Filled Core & & 70 & 21.1 & 0.44 & 82 & 7.6 \\
\hline \multirow{2}{*}{ Design 4} & Open Core & \multirow{2}{*}{35.8} & 73 & 29.3 & 0.56 & 46 & 7.6 \\
\hline & Foam- Filled Core & & 69 & 31.2 & 0.41 & 82 & 10.6 \\
\hline
\end{tabular}

The densification strain was smaller for foam-filled core structures because strut collapse reduced the available volume within which the foam resided. This causes the foam to compress beyond its own densification strain, and the stress it can support to rises rapidly. If we assume 
the stress in the foam is the same as that supported by the struts, the strain in the H130 foam at densification of the structure, $\varepsilon_{\text {foam }}$, varies from $72-87 \%$. The predicted densification strain of the foam-filled corrugation is simply the product of the densification limit of the foam (at the equivalent stress levels during densification of the corrugated structure (i.e. $\varepsilon_{\text {foam }}$ ) with the densification strain of the open cell corrugated structure, Equation (7.10).

$$
\varepsilon_{D}=\varepsilon_{\text {foam }}\left(1-\frac{t}{l \sin \omega}-\frac{1-x}{\sin \omega} \sqrt{1-\left(\frac{\cos \omega-x}{1-x}\right)^{2}}\right)
$$

The predicted densification-relative density relation (curve) and specific predictions for each structure (open green diamonds) agree well with the experimental data, Figure 76.

The densification strain models for the open core (Equation (7.10)) and foam-filled core (Equation (7.11)) can be simplified using the observed average hinge value of $\mathrm{x}=0.7$ and intended design orientation angle of $\omega=60^{\circ}$ for all specimens. Simplified predictions are compared to the comprehensive densification models along with data measurements and shown in Figure 76. It can be seen that the simplified predictions agree well with the experimental data regardless of the small variation in the geometric parameters $\mathrm{x}$ and $\omega$, providing a useful predictive tool in design when $\mathrm{t}$ and 1 are the only primary variables.

\section{Energy Absorption Prediction}

The energy absorbed can be approximated with an energy absorption efficiency parameter. The energy absorption (per unit volume), U, of a core can be predicted from the 
product of the compressive strength, $\sigma$, of the core (predicted by Equations (7.6) and (7.7)), and the densification strain (given by Equations (7.10) and (7.11)) of the core.

$$
U=\xi \cdot \varepsilon_{D} \cdot \sigma
$$

The energy predictions for each of the four open and foam-filled core designs is overlaid on the data in Figure 77, and predicts the general trends of the experimental results quite well. Table 9 lists the predicted volumetric energy absorptions for each core design. We note that Divinycell $\mathrm{H} 130$ has a volumetric energy absorption of $1.1 \mathrm{MJ} / \mathrm{m}^{3}$ when compressed to its densification strain. The energy absorption of the hybrid structure was slightly higher than that of the sum of the foam and empty core components due to compression of the foam beyond its densification strain and stabilization of slender struts that failed by Euler (elastic) buckling.

\subsection{Multi-Tier Sandwich Panel}

Rear-face supported, multi-tier corrugated sandwich panels were chosen as the desired panel for high explosive blast analysis. A multi-tier structure was chosen to investigate the impulse against a sizeable threat. As a result, the quasi-static compressive strength and modulus for the multi-tier corrugated sandwich panel is investigated.

\subsubsection{Test Methodology - Multi-Tier}

Similar to the single tier corrugated sandwich panels, the multi-tier structures are investigated under quasi-static compression with a fully supported rear-face boundary condition. These tests differ from earlier tests, as there is no lateral confinement used throughout the testing process. This scenario is designed to reproduce the boundary conditions as tested in the high 
explosive blast tests. Figure 81 illustrates the quasi-static test used for the multi-tier corrugated sandwich panels.

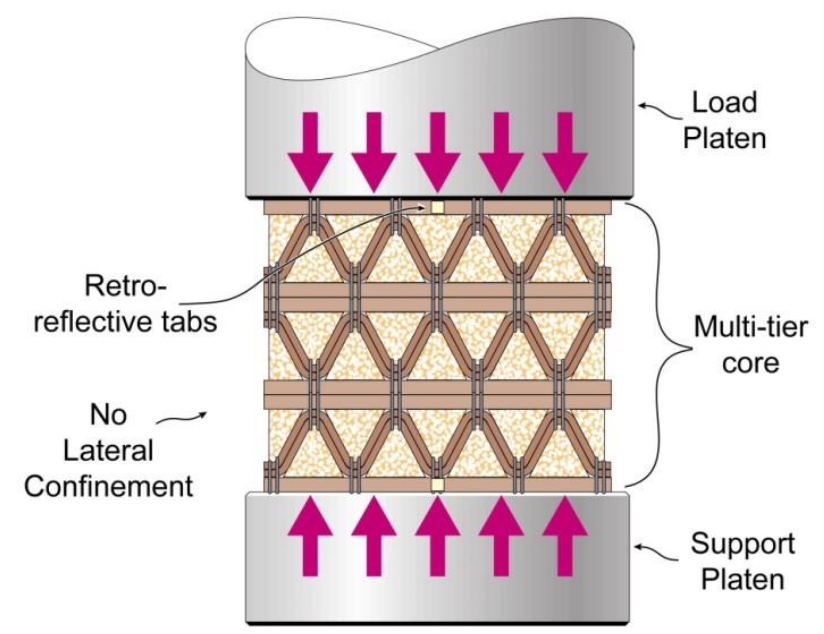

Figure 81: The compression set-up for the hybrid sandwich panel provides only a rear face boundary condition during testing.

Samples were constructed to be three tiers high with a width of four unit cells to provide a representative sample of the blast sample structure. Two relative densities (as calculated from the single tier, open core structures) were tested, including the $15 \%$ (1 laminate thick E-glass strut) and 27\% (2 laminates thick E-glass strut), both with foam cores intact and with an open (empty) core. All specimens were tested at an ambient temperature of $25^{\circ} \mathrm{C}$ and a strain rate of $10^{-3} \mathrm{~s}^{-1}$. Measurements were made using a screw-driven Instron universal mechanical test machine utilizing a $300 \mathrm{kN}$ load cell for nominal stress measurements and a laser extensometer to measure the core strain between the two facesheets.

\subsubsection{Experimental Results - Multi-Tier}

The compressive stress-strain responses of the $15 \%$ and $27 \%$ relative densities are shown in Figure 82 for representative samples of the foam-filled and open (empty) core structures. During initial loading, all samples deform in a linear elastic manner, reaching peak yield 
strengths at strain levels less than 5\%. It is observed that the stress-strain response varies by the initial failure. In the cases of observed nodal rotation, failure was observed to occur simultaneously in more than one tier, exhibiting a stress-strain response with reduced plateau strengths approximately maintained through loading until densification. However, variations that resulted from slight manufacturing difference or defects create structures with a slightly different strength bearing capabilities. If nodal alignment is correct, a cascading stress-strain response can be observed in the stress-strain, Figure 82(b), and visually seen in the compression of the open core structure in Figure 83. A cascading stress-strain response shows the effect of a slight strength differences in each core/tier. Each tier will fail as the critical strength of that core is reached, and the stress-strain response will follow the reduced strength plateau response as observed earlier until the strength grows large enough to fail the next core/tier. Finally, at the onset of densification, material consolidation changes the stress-strain response to rapid strain hardening as anticipated from the single tier results. Increases in the relative density of the structure show an increase in the observed peak strength and a decrease in the densification strain. Samples with the foam-filled cores showed a significant increase in energy absorption. A summary of the initial peak strength and plateau strengths through full compressive loading is given in Table 10. 

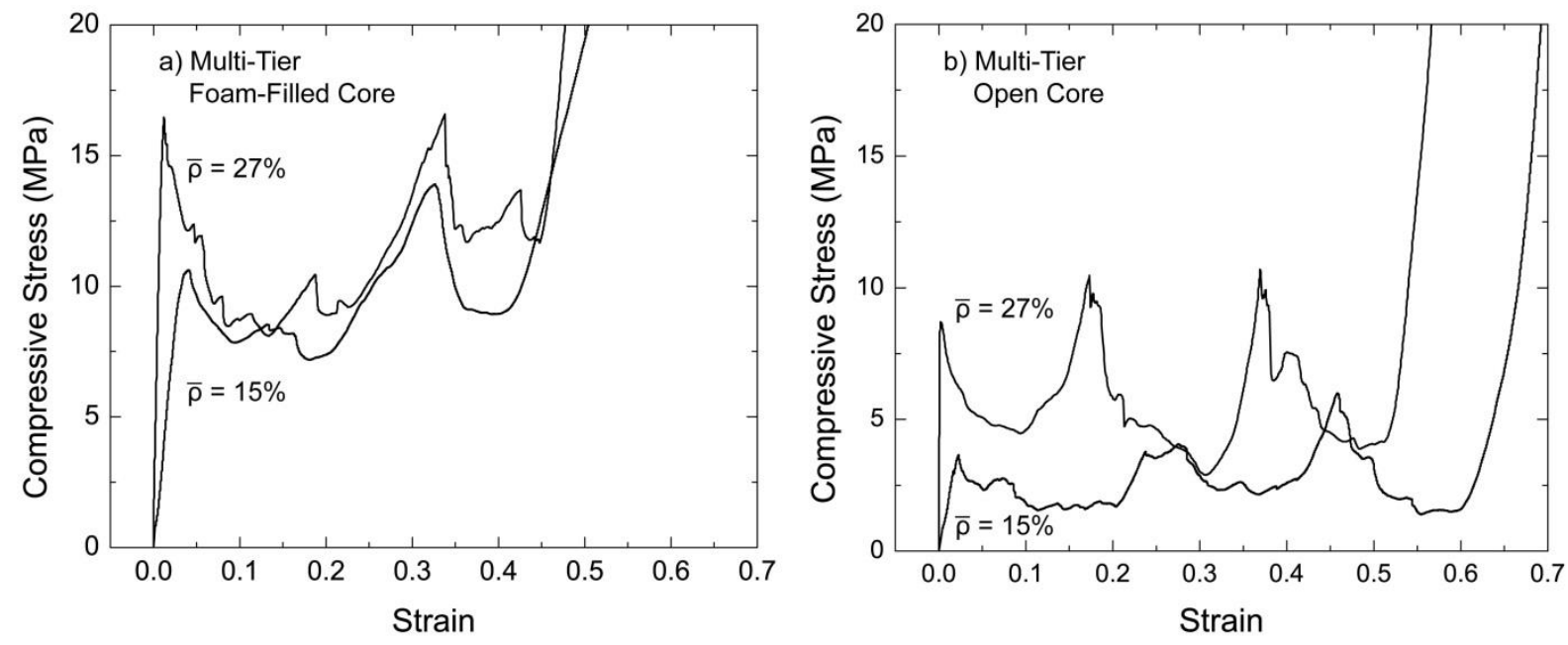

Figure 82: Compressive stress-strain response for multi-tier (3-tier) glass-epoxy corrugated sandwich panel structures for the a) Divinycell H130 foam-filled structure and b) open core structure.

Table 10: Summary of the strength data for the foam-filled and open core multi-tier panels.

\begin{tabular}{|c|c|c|c|c|}
\hline Core Design & $\begin{array}{l}\text { Predicted } \\
\text { Open Core } \\
\text { Relative } \\
\text { Density }(\%)\end{array}$ & $\begin{array}{l}\text { Core } \\
\text { Type }\end{array}$ & $\begin{array}{c}\text { Initial Peak } \\
\text { Strength } \\
(\mathrm{MPa})\end{array}$ & $\begin{array}{c}\text { Plateau } \\
\text { Strength } \\
(\mathrm{MPa})\end{array}$ \\
\hline \multirow{2}{*}{ Design 3} & \multirow{2}{*}{$27 \%$} & Open Core & 8.7 & 6 \\
\hline & & Foam-Filled Core & 16.2 & 12 \\
\hline \multirow{2}{*}{ Design 2} & \multirow{2}{*}{$15 \%$} & Open Core & 3.6 & 4 \\
\hline & & Foam-Filled Core & 10.5 & 10 \\
\hline
\end{tabular}

Photographs taken during the compression of core Design $3(t / l=0.14)$ are shown for the foam-filled and open core structures, Figure 83. The foam-filled and open core structures were compressed to a strain of $50 \%$ with the associated core response captured. Initial failure varied depending on the imperfections present. If nodal misalignment imperfections are present, failure is observed to initiate at these positions by developing a nodal rotation. Before the nodal 
rotation leaves the elastic regime, buckling failure in the struts is observed and partially created by the bending forces of the nodal rotation. Without nodal misalignment, strut failure was primarily due to strut buckling, including plastic microbuckling for the thicker struts and Euler buckling for the thinner struts. Shear failure at the outer strut nodal attachments is also observed, allowing the struts to break away and no longer support load. This has the effect of redistributing the load among the remaining struts in the core, thereby decreasing the load carrying capability. 


\section{Foam-Filled Core Open Core}

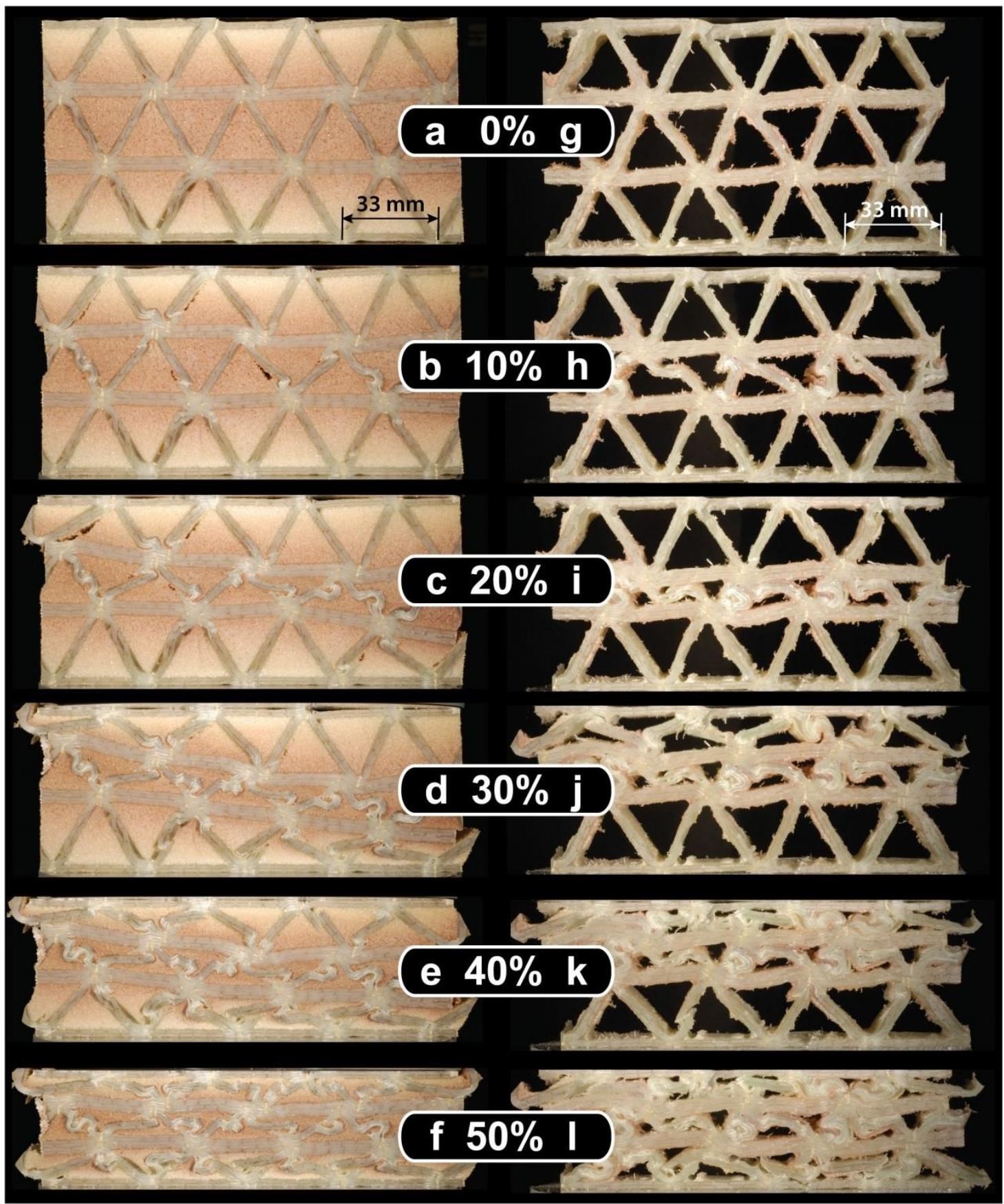

Figure 83: Photographs taken during compression of the multi-tier corrugated sandwich structure (Design 3, t/l=0.14). Many failure modes are observed including truss Euler buckling, microbuckling, nodal rotation, outer edge nodal shear, and matrix crazing. Parts a-f correspond to the foam-filled structure and parts g-l correspond to the open core structure. 
The measured modulus values varied significantly with the cores. The foam-filled cores exhibited moduli ranging from $0.4-1.5 \mathrm{GPa}$ and the open cores ranged from $0.2-2 \mathrm{GPa}$, comparable to the single-tier modulus results. The discovery of the effect that imperfections in facesheet waviness have on the elastic modulus for the single-tier cores helps explain the low and variable moduli observed in the multi-tier structure. The multi-tier corrugated structures exhibited the same imperfections, Figure 83, for both the foam-filled and open cores and justifies the lower than predicted modulus.

The initial (peak) strength for the foam-filled and open core structures were based on a limited number of samples and only tested for $15 \%$ and $27 \%$ relative densities. The peak strengths for the foam-filled core varied from 10.5 to $16.5 \mathrm{MPa}$ as the relative density increased from 15 to $27 \%$. The strength of the open core samples was generally less, varying from 3.6 to 8.6 $\mathrm{MPa}$ over the same relative density range. It is observed that the peak strength of the foamfilled cores was 7-8 MPa higher than that of the open core structure, greater than the $3 \mathrm{MPa}$ foam core that was removed suggesting a synergistic effect. The densification strains observed in the multi-tier sandwich structures ranged from $42-47 \%$ for the foam-filled cores and $54-63 \%$ for the open core structures.

\subsection{Comparison with a Foam Material Response}

The hybrid foam glass-epoxy composite corrugated core cellular structure sandwich panel is a design refinement from the original foam sandwich presented by Latourte et al. [11]. Maiti, Gibson and Ashby $[26,58]$ have developed empirical relations to predict the behavior of such a foam structure. The mechanical tests reveal that this structure is not entirely governed by 
stretch-dominated failure modes. Therefore, for comparison the response of the single-tier, hybrid corrugated structure is compared to the empirical relation established for bendingdominated foam materials.

The empirical relationship for the dependence of the compressive modulus of foams, $\mathrm{E}_{\text {foam }}$ on the relative density, proposed by Maiti et al. is;

$$
\frac{E_{\text {foam }}}{E_{s}}=C_{1}(\bar{\rho})^{n_{1}}
$$

where $E_{f o a m}$ is the compressive modulus of the foam/core structure, $E_{s}$ is the modulus of the solid material that forms the cell walls, $\bar{\rho}$ is the foam relative density and $C_{l}$ and $n_{l}$ are constants. The empirical results of Maiti, Gibson, and Ashby [58] indicate that $C_{1}=1$ and $n_{1}=2$ for the response of bending-dominated foam and $C_{1}=1 / 3$ and $n_{1}=1$ for stretch dominated failure. The closed cell DIAB Divinycell H-series foam was well fitted by both the bending-dominated and stretch dominated predications at the relative densities tested. The experimental modulus data was plotted as a ratio of the through-thickness modulus of the corrugated core to the experimentally measured modulus of the composite struts (i.e. solid material) corresponding to the core design. Core designs 2 and 3 exhibited strut fiber volume fractions ranging from 30$35 \%$ with an average strut modulus, $\mathrm{E}_{\mathrm{s}}$, of $16 \mathrm{GPa}$ and core designs 1 and 4 exhibited strut fiber volume fractions ranging from $35-40 \%$ yielding an average strut modulus of $19 \mathrm{GPa}$. The experimental data showed little difference in the modulus response between foam-filled and open core structures, but does show a significant difference between the unit cell and multi-cell structures. The unit cell structures are well fitted by the empirical Equation (7.13) using $\mathrm{n}_{1}=0.8$ and $\mathrm{C}_{1}=1.5$, while the multi-cell structures are well fitted with constants $\mathrm{n}_{1}=0.3$ and $\mathrm{C}_{1}=2$. 
These empirical fits indicate that no structure exhibits a purely stretch dominated response. The multi-cell core structures exhibit a bending-dominated response, as expected due to the loading imperfections. The facesheet imperfections that are removed in the unit cell structure changes the response to more closely fit a stretch-dominated failure response, but does not precisely matching Maiti and Gibson's models $[26,30,58]$. This result shows improvements in the structure can significantly change the response to behave less like a bending dominated foam and more as a stretch-dominated cellular structure. This result would warrant the further analysis of this structure at a greater range of relative densities to further investigate the response. While the predicted modulus behavior on relative density developed in the pin-jointed model, Equation 7.3, predicts a linear response with good agreement, the empirical fit prediction indicates the pinjointed model may not work at a full relative density range and may fail at low $(\bar{\rho}<15 \%)$ and high $(\bar{\rho}>45 \%)$ relative densities. These structures were outside the scope of this research. These results are summarized in Figure 84. 


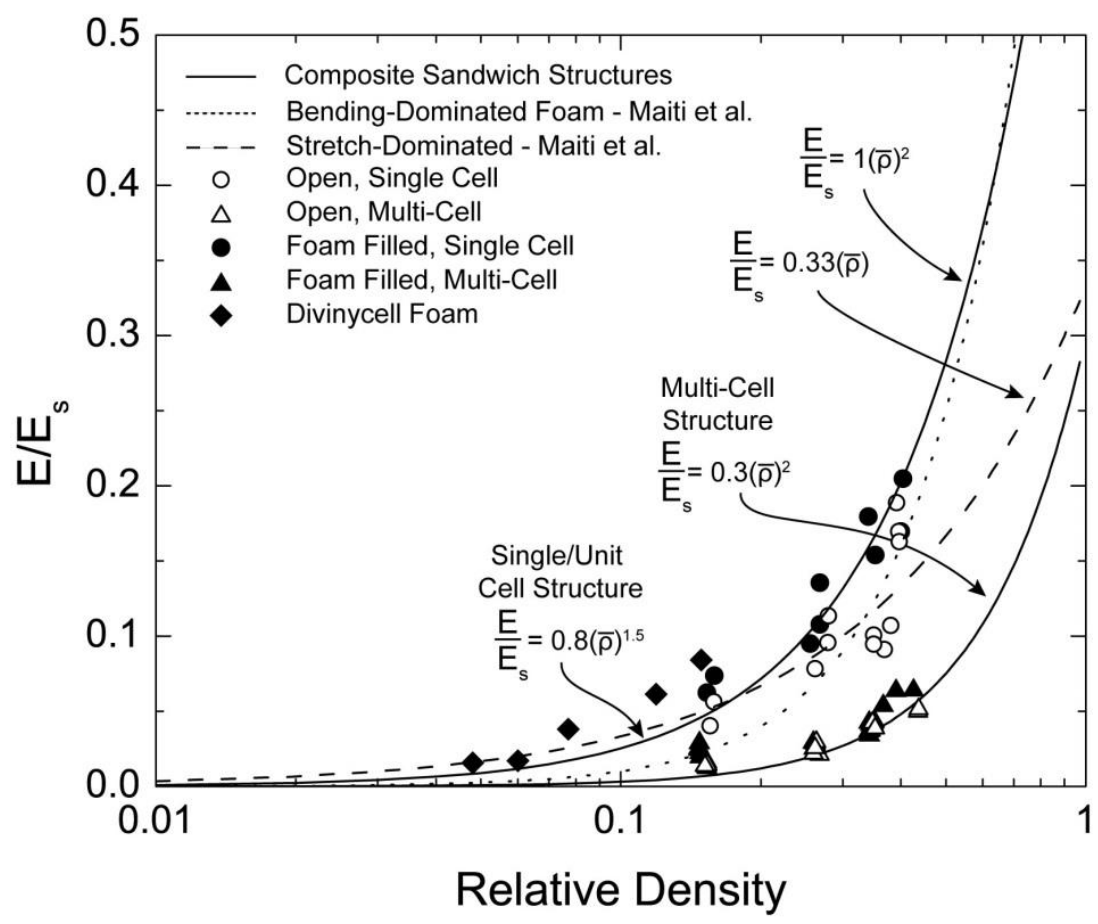

Figure 84: The modulus response of the corrugated structures compared to Maiti, Gibson, and Ashby's [58] bending-dominated foam model. Modulus is normalized with the bulk material modulus of the

The compressive strength empirical relationship of foams, $\sigma_{\text {foam }}$ on the relative density is:

$$
\frac{\sigma_{\text {foam }}}{\sigma_{y s}}=C_{2}(\bar{\rho})^{n_{2}}
$$

where $\sigma_{\text {foam }}$ is the compressive strength of the foam structure, $\sigma_{y s}$ is the yield/critical strength of the composite/solid material that form the cell walls, $\bar{\rho}$ is the foam relative density and $C_{2}$ and $n_{2}$ are constants of proportionality. The yield strength of the composite cores was observed to vary by the slenderness ratio and fiber volume fractions, and therefore, each core design is normalized by the average yield strength of the struts for a given core design, and shown in Table 8. Maiti, Gibson, and Ashby [58] indicate that for a plastic collapse bending-dominated foam, $\mathrm{C}_{2}=0.3$ and $\mathrm{n}_{2}=1.5$ and for stretch dominated foams the relationship to relative density is 
linear $\left(\mathrm{n}_{2}=1\right)$ and the constant of proportionality, $\mathrm{C}_{2}$, can vary from 0.3 to 0.7 . The closed-cell

Divinycell H-series foam fits Maiti et al. bending-dominated empirical relation, but the strength results of the core are not as clear. The data showed little difference between the unit cell and multi-cell structures, but a slight difference was observed between the foam-filled and open core structures, with the open core structures exhibiting a lower strength. The best parameter fits for the foam-filled cores was $\mathrm{C}_{2}=0.8$ and $\mathrm{n}_{2}=1$ and for the open cores, $\mathrm{C}_{2}=0.8$ and $\mathrm{n}_{2}=1.2$. While the relation is not observed to be strictly linear with relative density, the trend is similar to stretchdominated failure. These results are summarized in Figure 85.

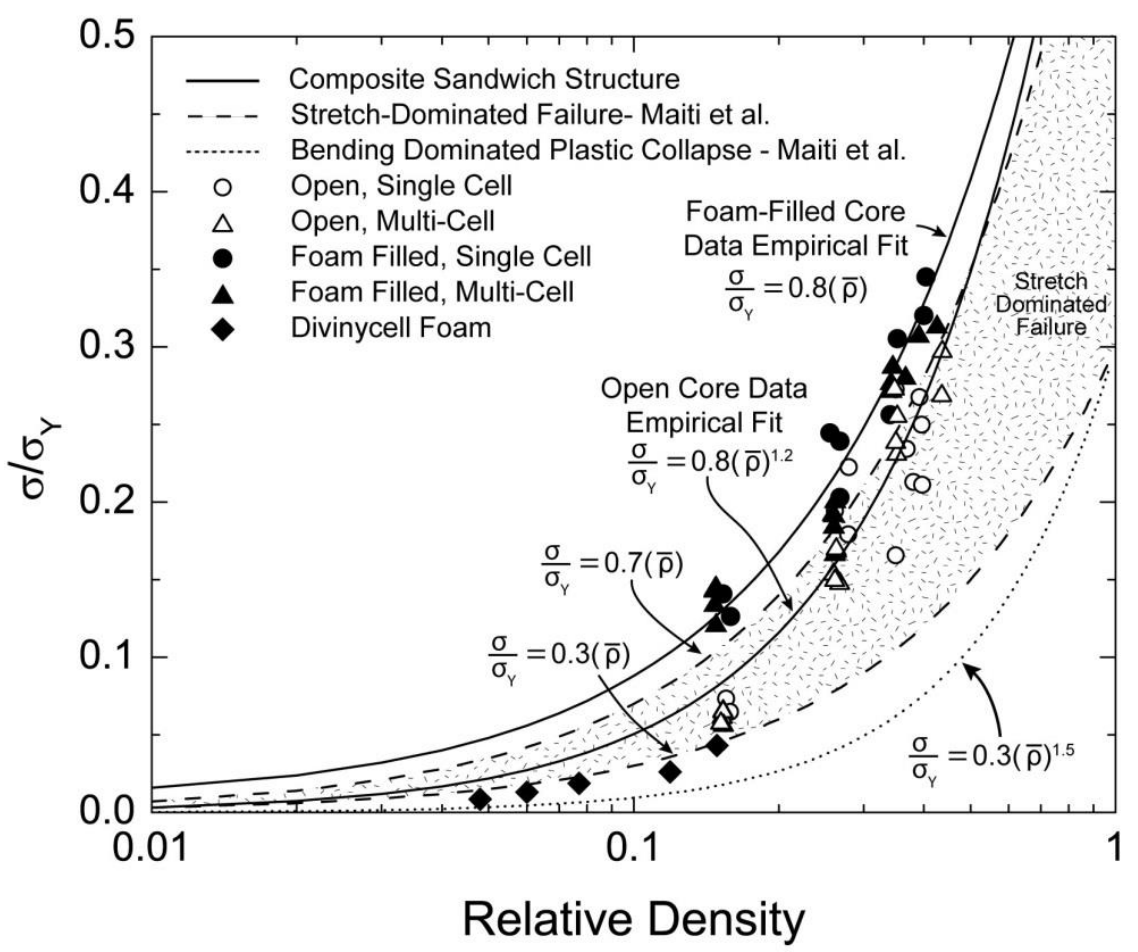

Figure 85: The strength response of the corrugated structures compared to empirically determined bending-dominated and stretch-dominated foam models developed by Maiti, Gibson, and Ashby's [58]. 
Finally, a simple linear relation of densification strain upon relative density has been proposed for closed cell foams by Maiti, Gibson and Ashby [26, 58];

$$
\varepsilon_{D}=1-C_{3} \bar{\rho}
$$

where $\varepsilon_{\mathrm{D}}$ is the densification strain, $\bar{\rho}$ is the foam relative density and $C_{3}$ is a constant of proportionality. Maiti et al. propose that $C_{3}=1.4$ for closed cell foams. For the range of Divinycell foams tested, as shown in Figure 76, the data is well predicted with $\mathrm{C}_{3}=6$, much different than the empirical model. However, it is expected that that the densification strain will converge to zero as $\bar{\rho}$ tends to unity. Equation (7.15) does not predict this boundary condition requirement and therefore is likely to be valid only for a given range of relative densities. 


\section{Chapter 8}

\section{Hybrid Corrugated Sandwich Panel}

\section{Dynamic Compression and High-Explosive Blast Loading}

\subsection{Dynamic Compressive Loading}

It has been demonstrated that sandwich panels show resistance to blast loading through their flexural strength and fluid-structure interaction effects $[13,123]$. In a blast event, the core must be sufficiently stiff and strong to resist compressive strain and failure that would decrease facesheet separation and reduce flexural strength. Further, blast armor technology seeks to be both lightweight and strong to provide a viable protection that can actually be used. This chapter investigates the behavior of the hybrid foam glass-epoxy composite corrugated cellular structure sandwich panel under compressive dynamic and high-explosive blast loading.

\subsubsection{Test Methodology}

The dynamic out-of-plane compressive responses of the corrugated sandwich panel, the E-glass core strut, and Divinycell H130 foam were measured from a series of direct impact tests of a high speed cylindrical projectile through the use of a Kolsky Bar. A Kolsky Bar, also known as a Split Hopkinson Pressure Bar (SHPB) is a characterization tool for measuring the mechanical response of materials or structures at high strain rates [124]. The Kolsky Bar is a 
solid cylindrical bar (usually made of aluminum or steel) instrumented with strain gauges to record localized strain at a specific position in the bar as stress waves propagate through the material. The specimens were mounted centrally on the stationary Kolsky Bar with the sandwich panel rear face coincident with the end of the Kolsky Bar. A cylindrical projectile, the same diameter as the Kolsky Bar, is fired from the barrel of a gas gun towards the sandwich panel to provide dynamic compressive loading on the structure, illustrated in Figure 86. The force transmitted by the specimen was measured as a function of time for a range of impact velocities of the striker bar.

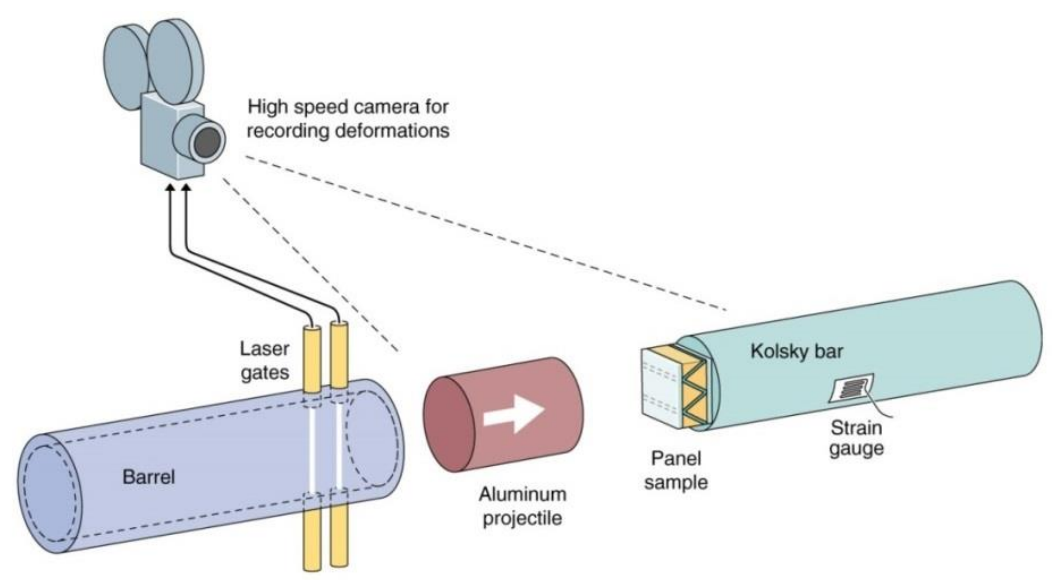

\section{Figure 86: Illustration of the dynamic compressive loading of the corrugated sandwich panels. A Kolsky Bar is used for rear face pressure measurements as a cylindrical projectile impacts the sample.}

A cylindrical aluminum bar, $2.0 \mathrm{~m}$ in length, was used to measure dynamic throughthickness, rear face pressures from the structure impacting the end of the bar. Measured by diametrically opposed axial strain gauges placed approximately 10 diameters from the impact end of the bar, strains could be calculated through the use of a half-Wheatstone bridge amplified to provide a high frequency output signal. The bridge system was calibrated dynamically over the range of strains measured during the experiments and was accurate to within $3 \%$. Two 
separate Kolsky Bars were used for testing the corrugated core specimens and the material test coupons, Figure 87, (i) a $76.2 \mathrm{~mm}$ diameter aluminum alloy (AL-P6082-T6, yield strength $310 \mathrm{MPa}$ ) bar was used in the corrugated core tests while (ii) $28.5 \mathrm{~mm}$, diameter maraging steel (yield strength $>1000 \mathrm{MPa}$ ) bar was used in the material coupon tests. These two different diameter bars were used to minimize the contact area differences between the specimens and the Kolsky Bars. The longitudinal elastic wave speed was measured at $5092 \mathrm{~ms}^{-1}$ in the aluminum alloy bar, and $4906 \mathrm{~ms}^{-1}$ in the maraging steel bar. Taking into account that the strain gauges are placed approximately 10 bar diameters from the impacted end, this gives a time-window of $487 \mu$ s and $781 \mu$ s in the aluminum and steel Kolsky Bars, respectively, before elastic stress wave reflections from the rear end of the Kolsky Bar return to the strain gauge position [37].

The compressive tests were conducted on "Design 1" corrugated sandwich panels, Table 2, which possessed an open core relative density of $40 \%$. These samples were two unit cells in width, measuring $53 \mathrm{~mm} \times 53 \mathrm{~mm}$. Samples tested included both foam-filled and open core variants. The size of these samples made them highly vulnerable to nodal shear failure between the core strut and facesheet. To avoid this failure mode and investigate the purely compressive response of the corrugated core, a steel confinement rig is used to maintain nodal alignment, as illustrated in Figure 87. The confinement rig had the negative effect of restricting the compression of the sandwich core to a nominal compressive strain of less than 70\% [37]. This was deemed acceptable since the densification strain of the corrugated sandwich panel was approximately $40 \%$.

In the experiments on sandwiches conducted at low velocity $\left(v_{o} \leq 50 \mathrm{~ms}^{-1}\right)$ a striker of mass $M=2.5 \mathrm{~kg}$ was employed, while a striker of mass $M=0.75 \mathrm{~kg}$ sufficed for the high 
velocity $\left(v_{o}>50 \mathrm{~ms}^{-1}\right)$ experiments. For the materials test, a striker of mass $M=0.1 \mathrm{~kg}$ was sufficient for all velocities. High-speed photographs taken during these experiments show that these striker masses are sufficient to provide almost constant velocity compression for nominal compressive strains of up to $40 \%$. The striker was given the required velocity by firing it from a gas gun of barrel length $4.5 \mathrm{~m}$. No sabot was employed as the cylindrical strikers had a diameter equal to the inner diameter of the gun barrel and equal to the diameter of the Kolsky Bar. The impact experiments were performed at velocities ranging from approximately $10 \mathrm{~ms}^{-1}$ to $150 \mathrm{~ms}^{-1}$. The velocity of the projectile was measured at the exit of the barrel using laservelocity gates and the impacted end of the Kolsky Bar was placed $100 \mathrm{~mm}$ from the open end of the gun barrel [37].

(a)

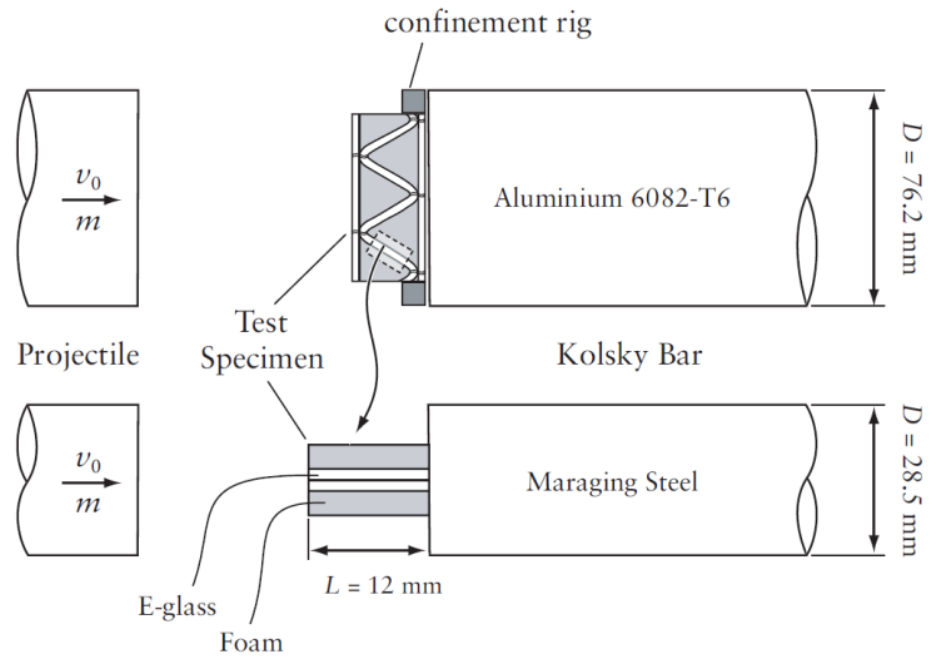

Figure 87: Sketches of the Kolsky Bar setup arrangements for dynamic testing of the (a) corrugated core specimens and (b) the E-glass test coupons cut from the struts of the corrugated cores [37].

The response time and accuracy of the measurement system was gauged from a series of calibration tests, as shown in the measured stress-time plot, Figure 88, for a representative test on the maraging steel Kolsky Bar. A maraging steel striker of diameter $28.4 \mathrm{~mm}$ and length 
$460 \mathrm{~mm}$ was fired at the Kolsky Bar at a velocity $v_{o}=6.6 \mathrm{~ms}^{-1}$. With time $t=0$ corresponding to the instant of impact, the stress pulse arrives at the gauge location at $t=58 \mu$ s. Elastic wave theory predicts that the axial stress in the bar is $\rho c_{\text {long }} v_{o} / 2=131 \mathrm{MPa}$ [37], where $\rho$ and $c_{\text {long }}$ are the density and longitudinal elastic wave speed of steel respectively. The measured peak value of the stress is within $1 \%$ of this prediction. However, the measurement system has a finite response time, with the stress rising to this peak value in approximately $13 \mu \mathrm{s}$ (see the insert in Figure 88). This rise time places an operational limit on measuring the dynamic response of the specimens. It becomes significant at the higher velocities because significant compression of the specimen is achieved within the first $5 \mu \mathrm{s}$. The measured stress in the calibration test drops back to zero at $t=273 \mu \mathrm{s}$ when the reflection of the elastic wave from the rear end of the Kolsky Bar reaches the strain gauges [37].

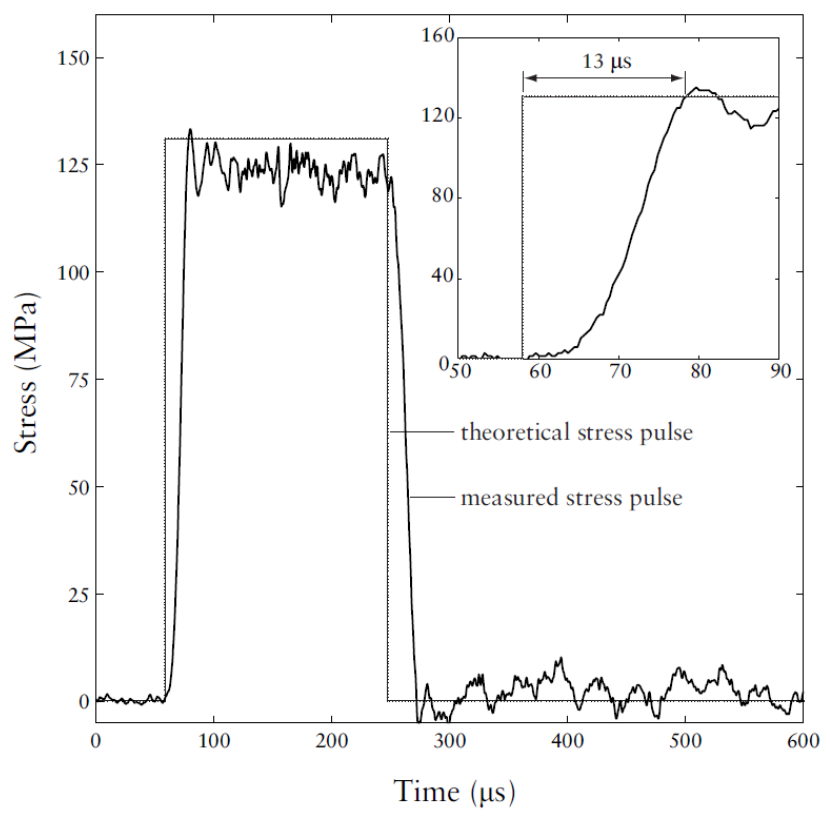

Figure 88: Stress versus time history measured in the $28.5 \mathrm{~mm}$ diameter maraging steel Kolsky Bar during a calibration test in which a $460 \mathrm{~mm}$ long steel striker $(28.5 \mathrm{~mm}$ diameter) was fired at Kolsky Bar at $v_{0}=6.6 \mathrm{~ms}^{-1}$. The theoretical prediction based on $1 D$ elastic wave theory is included in the figure along with an inset that magnifying the early time history of the stress pulse [37]. 


\subsubsection{Experimental Results - Strut}

The peak quasi-static strength of the open core sandwich panel ranged from 100-260 MPa depending on initial average fiber misalignment. All samples are shown to fail by plastic microbuckling with slenderness ratios $(\mathrm{t} / \mathrm{l})$ above 0.1 , Figure 63 . The measured dynamic compressive responses of a $12 \mathrm{~mm}$ in length E-glass strut specimens cut from the strut walls is plotted in Figure 89 for impact velocities ranging from $25 \mathrm{~ms}^{-1} \leq v_{o} \leq 150 \mathrm{~ms}^{-1}$. The compressive responses are plotted in terms of the nominal stress as measured at the back face of the specimen (i.e. the non-impacted end of the specimen) versus the applied nominal strain $v_{o} t / L$, where $t$ is the time measured after the initiation of deformation. The measured peak stress $\sigma_{f}$ is seen to increase to $400 \mathrm{MPa}$ at $v_{o}=150 \mathrm{~ms}^{-1}$ [37].
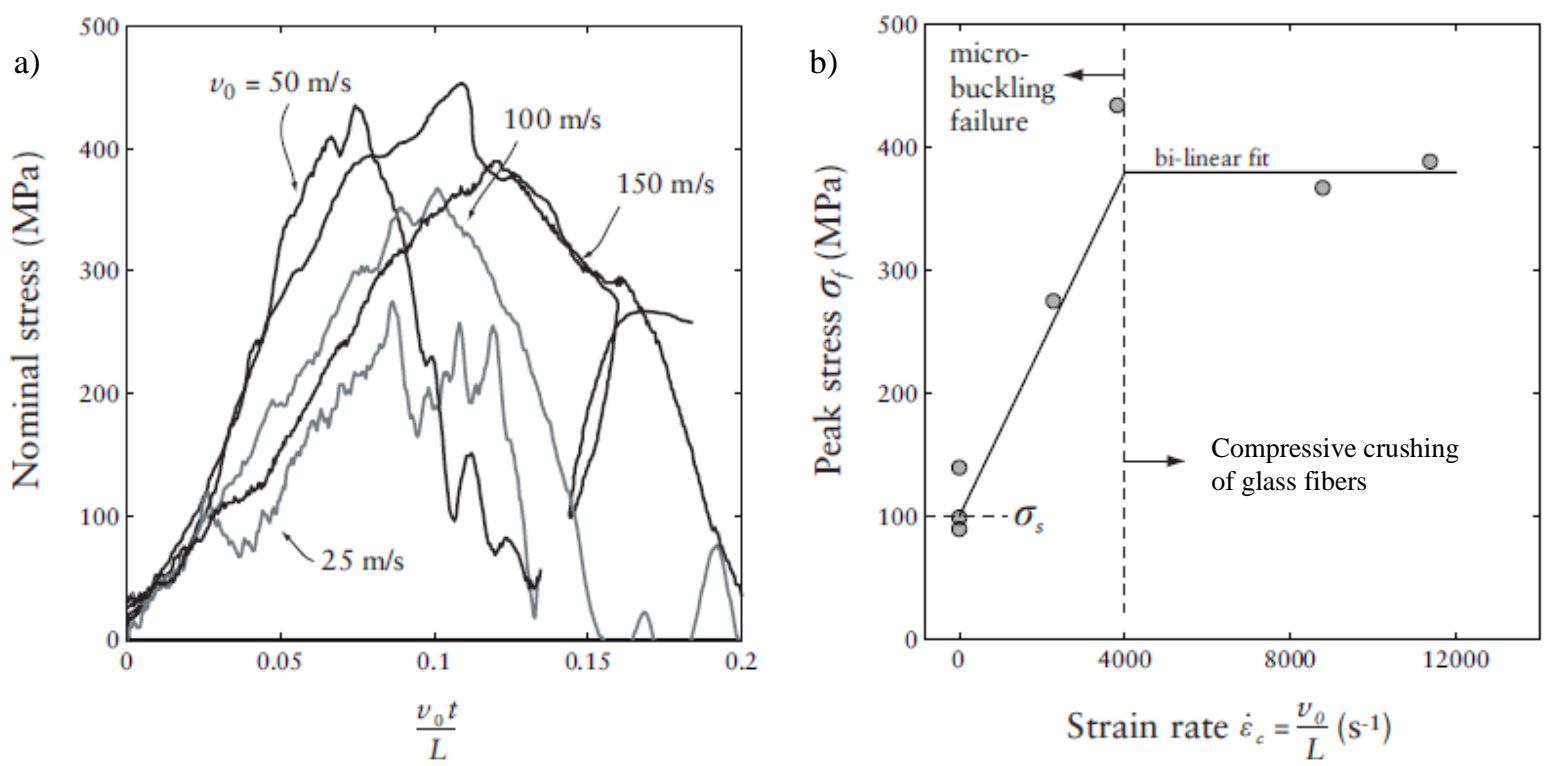

Figure 89: The measured dynamic stress versus nominal strain responses of the E-glass material coupons for a range of impact velocities. (b) Summary of the measured peak stresses E-glass specimens as a function of applied strain rate. The compressive response of the E-glass composite was measured along the weft fiber direction [37]. 
Given the measured Young's modulus $E_{s}$ of the E-glass composite, it is anticipated that the longitudinal elastic wave speed for the E-glass composite is about $4000 \mathrm{~ms}^{-1}$. Thus, within $12 \mu$ s, about four elastic reflections take place in the $L=12 \mathrm{~mm}$ specimen. It is therefore reasonable to assume that the specimen is in axial equilibrium at the instant that the peak stress is measured, and that the measured peak strength is a material property independent of the specimen dimensions. The measured peak strengths, $\sigma_{f}$, is plotted as a function of the applied nominal strain rate $\dot{\varepsilon} \equiv v_{o} / L$ in Figure 89 . The measurements are well-fitted by a bilinear curve with the peak strength increasing linearly with strain rate for $\dot{\varepsilon} \leq 4000 \mathrm{~s}^{-1}$ and reasonably rateindependent at higher values of $\dot{\varepsilon}$ [37]. Increasing the applied strain rate results in the stabilization of the micro-buckle failure mode, resulting in the peak strength increasing with $\dot{\varepsilon}$. Stabilization can be due to either (i) inertial stabilization of the buckling or (ii) matrix strain rate effects. Theoretical work by Fan and Slaughter [125] suggests that inertial effects become significant for strain rates greater than about $4000 \mathrm{~s}^{-1}$. Therefore, the measured rate sensitivity is likely due to matrix strain rate effects. This maximum peak strength is not set by microbuckling of the fibers but rather by the compressive crush/yield strength of glass fibers. This is rationalized as follows: The volume fraction of fibers in the composite is about $40 \%$ and only about $50 \%$ of the fibers are oriented parallel to the direction of loading. With an estimated Eglass virgin fiber compressive strength of $4 \mathrm{GPa}^{5}[101]$ and a readily seen $50 \%$ reduction in fiber strength during processing [22], this implies a crushing/yield strength of approximately 400 $\mathrm{MPa}$, equal to the compressive crush strength of glass and our experimental observations.

\footnotetext{
${ }^{5}$ This value is an estimate provided in CES EduPack 2011 and validation has not occurred because of the difficult in measuring the compressive strength of a fiber.
} 


\subsubsection{Experimental Results - Single-Tier}

The dynamic deformation process of the corrugated structures was taken using a highspeed Phantom V12 camera to observe failure during loading and measure the associated strain.

The deformation sequence for the foam-filled and open corrugated cores while being impacted at $150 \mathrm{~ms}^{-1}$ is shown in Figure 90. Deformation of the corrugated cores at quasi-static strain rates and at an impact velocity of $50 \mathrm{~ms}^{-1}$ look similar, showing failure occurring throughout the entire strut. Core deformation at an impact velocity of $v_{o}=150 \mathrm{~ms}^{-1}$ shows a marked difference. At this high velocity, deformation is more localized near the impacted face with the corrugated core "stubbing" against the impacted face. This deformation mode was also observed in metallic corrugated cores by Tilbrook et al. [126]. Given this highly localized deformation near the impacted face we anticipate that the stresses on the impacted face are higher than the stresses measured at the rear face (i.e. the specimen is not in axial equilibrium in line with the finite element calculations of Tilbrook et al. [126]). These differences between the stresses on the impacted and rear surfaces cannot be measured in this direct Kolsky Bar set-up as the inertia of the impacted face-sheet dominates the measurements; see Tilbrook et al. [126] for further discussion. The localized deformation mode was observed only for $v_{o} \geq 150 \mathrm{~ms}^{-1}$; and at the lower impact velocities the deformation was reasonably uniform through the core, suggesting that the specimens were in axial equilibrium at lower impact velocities [37]. 
(a) Filled
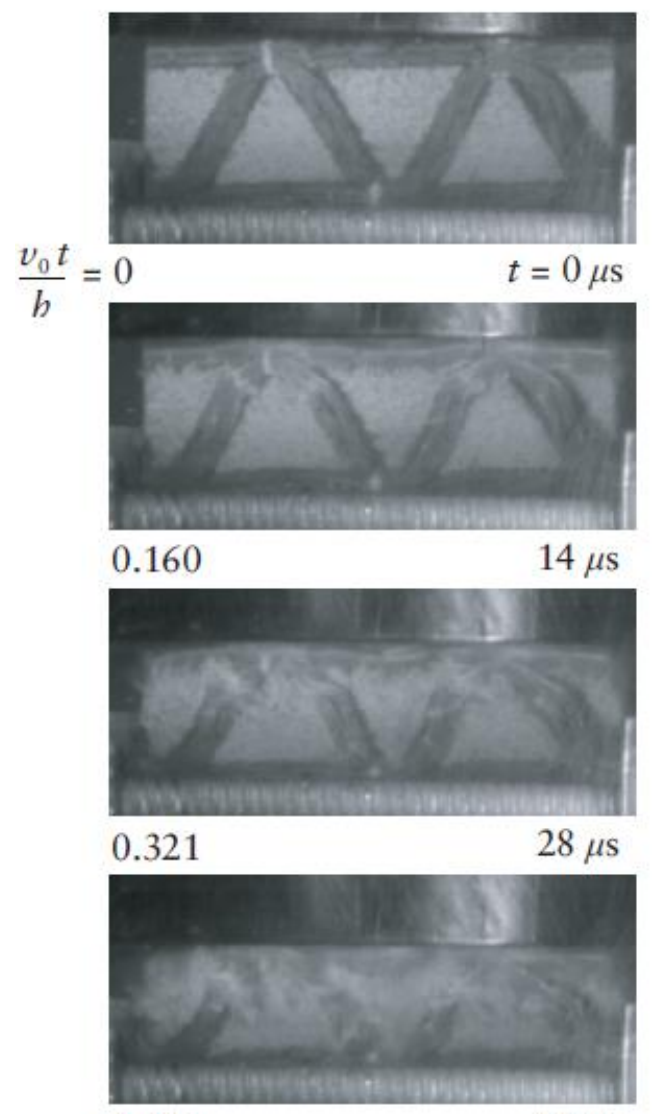

0.481

$42 \mu \mathrm{s}$ (b) Unfilled

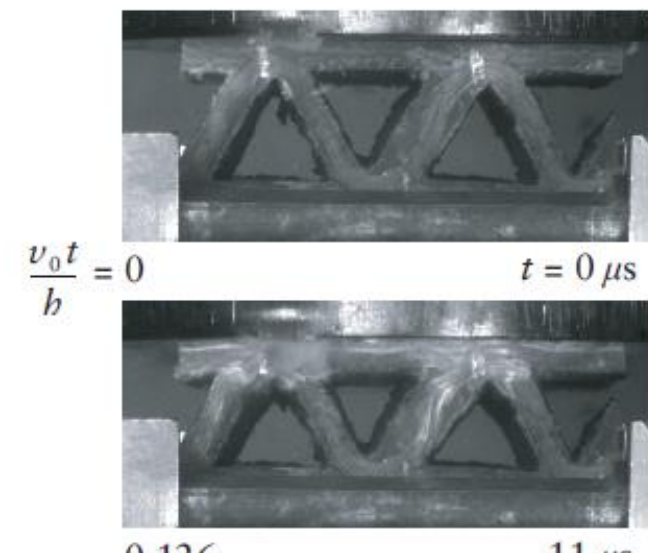

0.126

$11 \mu \mathrm{s}$

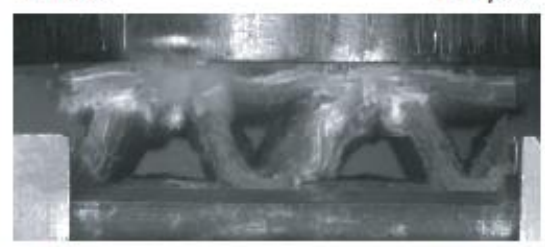

0.252

$22 \mu \mathrm{s}$

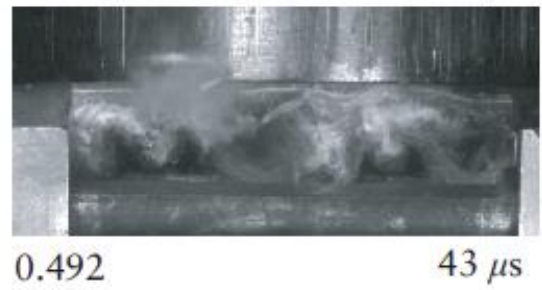

Figure 90: A montage of photographs showing the sequences of deformation in the (a) foam-filled and (b) open corrugated core specimens impacted at a velocity $v_{0}=150 \mathrm{~ms}^{-1}$. Impact occurs on the top face of the specimens in the photographs, illustrating the effect of dynamic impact and failure of the stress wave to propagate sufficiently fast enough through the sample.

The measured rear-face dynamic compressive stress responses of the foam-filled and open corrugated cores are plotted in Figure 91 as a function of nominal strain, $v_{o} t / h$, where time $t=0$ corresponds to the instant that the striker bar impacts the specimen. The responses of the foam-filled and open core specimens are almost identical with a the measured peak stress of approximately $90 \mathrm{MPa}$ over the velocity range $25 \mathrm{~ms}^{-1} \leq v_{o} \leq 175 \mathrm{~ms}^{-1}$. The peak stress is 
observed to occur at time $t$ in the range $15 \mu \mathrm{s}-18 \mu \mathrm{s}$. This is similar to the response time of the Kolsky Bar apparatus Figure 88. Similarly as before, these measurements cannot be used to infer the dynamic compressive moduli of the specimens [37].

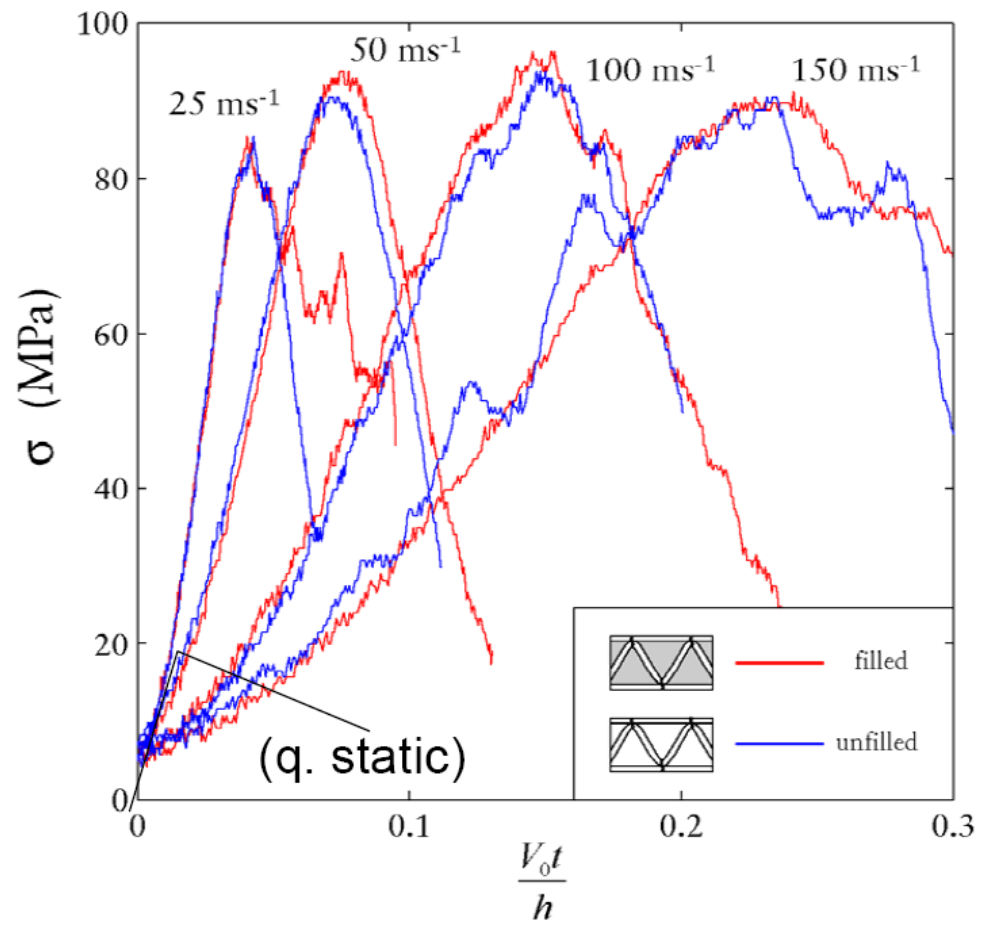

Figure 91: The measured dynamic stress versus nominal strain responses of the foam filled and open core corrugated specimens. The stresses are measured on the rear faces of the specimen opposite from the impacted face. Little effect is observed from the foam filed cores.

The rear-face measured peak stresses on the rear face of the foam-filled and open corrugated cores specimens are plotted in Figure 92 as a function of the impact velocity, $v_{o}$, and applied strain rate, $\dot{\varepsilon}_{c} \equiv v_{o} / h$, (shown on the upper scale). The dependence of the peak strengths of the corrugated cores on strain rate is similar to the parent strut wall material (Figure 89(b)), with the peak stress increasing with strain rate for $\dot{\varepsilon}_{c}<4000 \mathrm{~s}^{-1}$ and being reasonably rate insensitive at higher values of $\dot{\varepsilon}_{c}$. This clearly indicates that there exist two regimes of 
deformation: (i) micro-buckling of the strut wall for $\dot{\varepsilon}_{c}<4000 \mathrm{~s}^{-1}$ and (ii) compressive crushing of the glass fibers at higher strain rates. We note that the dynamic strength of the foam core (shown in Figure 92) is negligible compared to the strength of the corrugated core [37]. While there is no significant difference in the peak strength of the foam-filled and open core structure, the theoretical removal of the core to leaving a foam-only structure would have a tremendous effect. The strength of the foam-only core is approximately $10 \%$ of the hybrid structures. It is hypothesized that a negligible difference between the foam-filled and open core structures is due to the unique combination of dynamic loading and the geometry of this structure. Under such dynamic loads, failure is observed to occur closest to the front face/impact location. The composite core fails at the nodal points before loading of the foam even initiates, eliminating any potential impact of the foam within the cores.

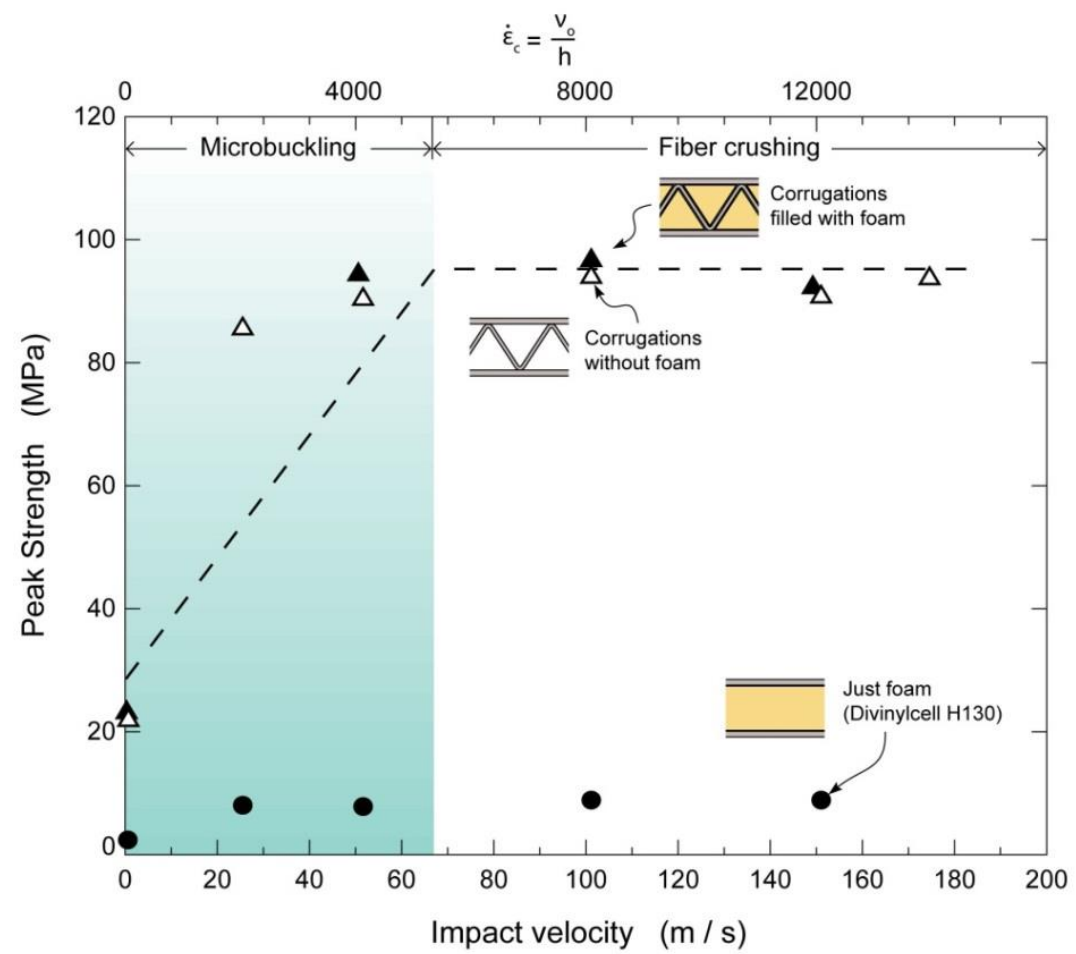

Figure 92: Summary of the measured peak stresses in the filled and unfilled corrugated core specimens as function of the impact velocity (lower $\mathrm{x}$-axis) and applied nominal strain rate (upper $\mathrm{x}$-axis). The measured peak stresses in Divinycell H130 foam are also included. 


\subsection{High-Explosive Compressive Blast Loading}

Cellular materials have widely been studied for structural use in armor, protective shielding, packaging, and energy absorption $[38,127]$. Studies have shown that low-density, cellular core sandwich panels show both an impulse mitigating and pressure reduction capability [128] when subjected to out-of-plane dynamic impact/impulse loading. The cellular sandwich panel response can limit the transmitted stress through the structure by absorbing energy applied to the front facesheet and mitigating the impulse through the collision response. The energy absorption response of stochastic materials, including both polymeric and metallic foams, is near ideal while exhibiting a bending-dominated failure under quasi-static loading. This energy absorption ability under quasi-static loading has led to the continued study of these materials under dynamic and blast loading, showing increases in energy absorption and impulse mitigation capability [38]. Interest has grown in the use of stretch-dominated failure mode cellular structures, including prismatic [123], corrugated [15], pyramidal [14], honeycomb [9], and laminated tubular core [128] structures, for impulsive loading. Underwater impulsive loading $[13,89,129]$ and high explosive blast testing has investigated the effects of core topology, materials (aluminum [130], steel [131, 132], and composites [11]), and boundary conditions (predominantly edge-clamped [15] and rear-face support [128]).

Determining the response of a material from high explosive testing is inherently dangerous and difficult to perform. The damage created by shockwave and high velocity fragments destroys equipment, test fixtures, and the samples being studied. Data acquisition is further challenging by the sensitivity to time and environmental conditions needed to collect useful information. As a result, a multitude of experimental methods have been employed from researchers trying to find a viable and repeatable method to obtaining data around high explosive 
testing. The simplest of experiments detonates an explosive charge near the surface of an edgeclamped plate for port-mortem analysis $[15,19]$, but complexity in analysis of structure performance is added with the fixture-to-sample interface (see Chapter 1). If a successful and repeatable method of clamping is created, impulse and energy transfer through the panel has been successfully measured with the use of a ballistic pendulum [85]. Out-of-plane response has been measured through the use of a restricted [90, 128] and unrestricted [133] vertical pendulums on bare charges and buried explosive charges. Vertical pendulums are used in industry to study the effects of buried explosives on vehicle hulls and armor technology. Through the use of high speed photography, piezoelectric sensors, accelerometers, and Kolsky Bars, success in characterizing a (relative) structure response has occurred in all loading scenarios mentioned, with limitations associated with each method.

\subsubsection{Test Methodology - High Explosive Blast Loading - Multi-Tier}

A dual-use vertical pendulum rig was designed to evaluate the out-of-plane, rear-face supported core response when loaded through high explosive sand blast loading. The vertical pendulum rig was designed to operate in two distinct modes: a fixed mode for measuring transmitted rear-face pressures and a sliding mode for measuring impulse mitigation. The vertical pendulum rig, pictured in Figure 93, consists of four aluminum bars, each $2 \mathrm{~m}$ in length, rigidly connected to aluminum plates on each end. The lower plate, measuring $304.8 \mathrm{~mm}$ (12 in) x $304.8 \mathrm{~mm}$ (12 in) x $25.4 \mathrm{~mm}$ ( $1 \mathrm{in})$, is the attachment point for a sample panel, via eight bolts. The aluminum bars pass through a series of bushings that connect and position the aluminum bars to a support frame, and the top of the bars are connected to a second rigid aluminum plate to provide upper end bar positioning support. Samples are designed to be $203.2 \mathrm{~mm}$ (8 in) x 203.2 
$\mathrm{mm}(8 \mathrm{in}) \mathrm{x} \sim 90 \mathrm{~mm}$ and connected to a rear mounting panel that matches the $304.8 \mathrm{~mm}$ (12 in) x $304.8 \mathrm{~mm}$ (12 in) rigid mounting plate and hole pattern. Finally, below the bars and test specimen is a steel containment box designed to provide the desired (and repeatable) environment for a buried explosive charge. The steel box is partially filled with dry, graded, sand that is used to support an explosive charge assembly made from explosives, detonators, and glass microspheres (used to control the explosive-sand-sample interaction with greater repeatability).

This study provided a preliminary investigation into the response of a three-tier hybrid corrugated composite sandwich panel. Numerous sample variables were investigated including relative density, effect of core foam removal, and front facesheet effects. Results are calibrated against a rigid aluminum solid block. Additionally, results are compared to pure Divinycell H130 foam panels. A multitude of variables were studied in the relationship of the explosive charge to the impulse transmission to a sample panel. This study varied the standoff distance between the explosive charge and sample front facesheet and explosive quantity. The depth of burial of explosive was fixed at $50.8 \mathrm{~mm}$ below the surface of the glass microspheres and fully saturated with water. These test conditions were experimentally determined to provide the greatest transferred impulse levels between the soil and the sample for the explosive amounts used for the tests. 


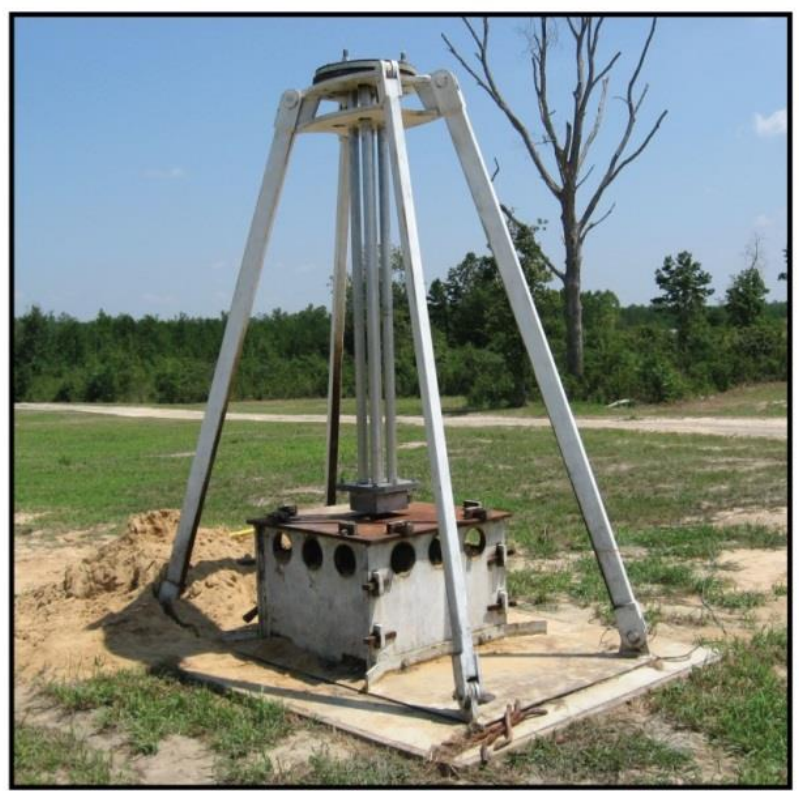

Figure 93: Photograph of the vertical pendulum rig used for rear-face supported testing of a core when loaded by high explosive driven high velocity sand.

The explosive charge assembly was constructed to provide transmission of a planer impulse to the sample. This is accomplished through the use of a flat sheet plastic explosive being detonated beneath a layer of glass microspheres which act as a "research grade" soil ejecta. The explosive charge assembly is illustrated in Figure 94. The explosive assembly is built around a thin, flat sheet of explosive called Detasheet. Detasheet is a flexible, rubberized, plastic explosive made from $63 \%$ pentaerythritol tetranitrate (PETN), $8 \%$ nitrocellulose, and 29\% of acetyl tributyl citrate (ATBC). To achieve a planer blast wave, the thin, flat explosive must be detonated almost simultaneously. A $254 \mathrm{~mm}$ (10 in) x $254 \mathrm{~mm}$ (10 in) explosive sheet was placed on a polyurethane base for support. Detasheet charges of $100 \mathrm{~g}, 200 \mathrm{~g}, 300 \mathrm{~g}$, and 400g are approximately $1 \mathrm{~mm}, 2 \mathrm{~mm}, 3 \mathrm{~mm}$, and $4 \mathrm{~mm}$ in thickness, respectively. To best achieve simultaneous denotation of the Detasheet, a series of five C4 explosive plugs were placed under the Detasheet in equal spaced square pattern with the fifth plug placed in the center of the charge, Figure 94. The C4 boosters were mounted within the $254 \mathrm{~mm}(10 \mathrm{in}) \times 254 \mathrm{~mm}(10 \mathrm{in}) \times 38.1$ 
$\mathrm{mm}$ (1.5 in) polyurethane foam support. Detonation is initiated to the each $\mathrm{C} 4$ booster through the use of Teledyne RISI Inc. RP-83 EBW detonators. The EBW detonators are mounted into a polystyrene foam support for the sole purpose of positioning the detonators beneath the $\mathrm{C} 4$ boosters.

Above the Detasheet explosive is a layer of $200 \mu \mathrm{m}$ diameter glass microspheres manufactured by Polysciences, Inc. The glass microspheres are used as a research grade soil ejecta to provide uniform sand particles free from contamination of sharp fragments or stones that can locally cause failure at impact. The microsphere layer is built-up above the Detasheet to stand $254 \mathrm{~mm}(10 \mathrm{in}) \times 254 \mathrm{~mm}(10 \mathrm{in}) \times 50.8 \mathrm{~mm}$ (2 in) tall, Figure 94, using $4.4 \mathrm{~kg}$ of microspheres. A square metal frame $254 \mathrm{~mm}$ (10 in) x $254 \mathrm{~mm}(10 \mathrm{in})$ is used to temporarily support the microspheres while water is added (1200 g) to provide full saturate.

The entire explosive charge assembly is built within the steel sand containment box to provide the necessary boundary condition, positioning, and support to the charge assembly. Soil is built-up under and around the charge assembly with continual realignment of the charge adjusted to assure the charge is centered under the pendulum, the correct standoff distance from the sample front surface is achieved, and the sand front/sample facesheet are oriented parallel. The support frame is removed from the experiment after full boundary condition soil positioning is complete to provide lateral support to the glass microspheres.

The final stage is to add a steel lid to the sand/charge containment box. The steel lid has a $203.2 \mathrm{~mm}(8 \mathrm{in}) \times 203.2 \mathrm{~mm}$ (8 in) aperture that is centered and aligned with the sample surface and microsphere ejecta. The lid is designed to only allow the glass microspheres to pass through to the sample and laterally spread the remainder of the soil outwards from the rig. 


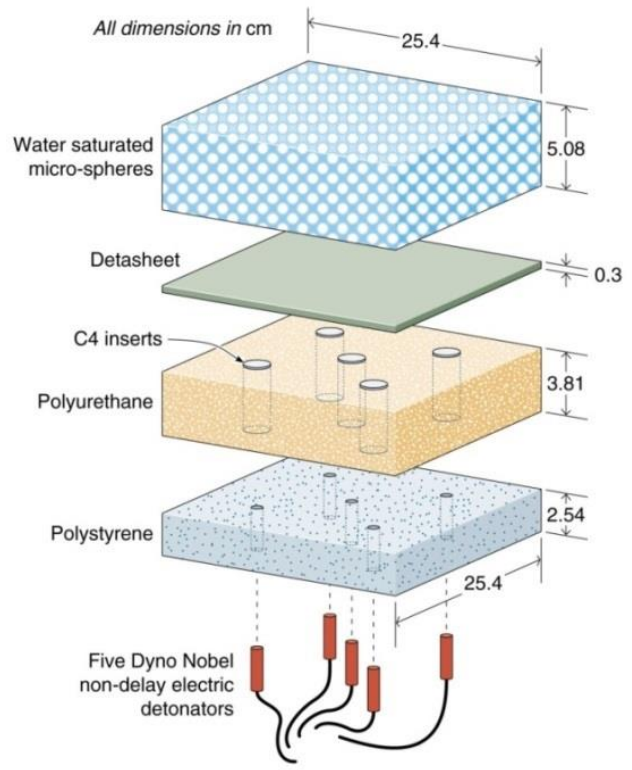

Figure 94: A diagram depicting the design of the explosive charge designed to accelerate water-saturated microspheres onto the front face of the specimens [128].

\section{Vertical Pendulum - Sliding Mode}

The basic operational mode of the vertical pendulum in sliding mode is to allow the aluminum bars to freely move up and down, allowing system to act as a vertical pendulum. A vertical pendulum allows for the applied impulses to be measured by the maximum height of travel of the pendulum, measured with photographic images taken during the test, Figure 95. As illustrated in Figure 96, the sample is mounted on the interconnected aluminum bars, aligned above the explosive charge assembly and soil containment box. The aluminum bars pass through a set of bushings which are fixed to the frame. The pendulum must be calibrated for the applied impulse that will be generated from the chosen explosive quantity and the standoff distance (distance between the sample and the explosive charge assembly) to allow the pendulum to operate within its given travel distance. The top of the aluminum bars are rigidly attached to an aluminum plate designed to precisely position the bars and provide a method to mount 
additional counterweights. Counterweights of either steel or aluminum were added in $7.2 \mathrm{~kg}$ increments. The counterweights are designed to limit the pendulums travel beyond the length of the aluminum slide bars. The minimum total mass of the vertical pendulum (minus the sample) is $57.8 \mathrm{~kg}$. This rig was routinely used with a total masses ranging from $80 \mathrm{~kg}$ to $120 \mathrm{~kg}$ for explosive Detasheet quantities ranging from 100 grams to 400 grams.
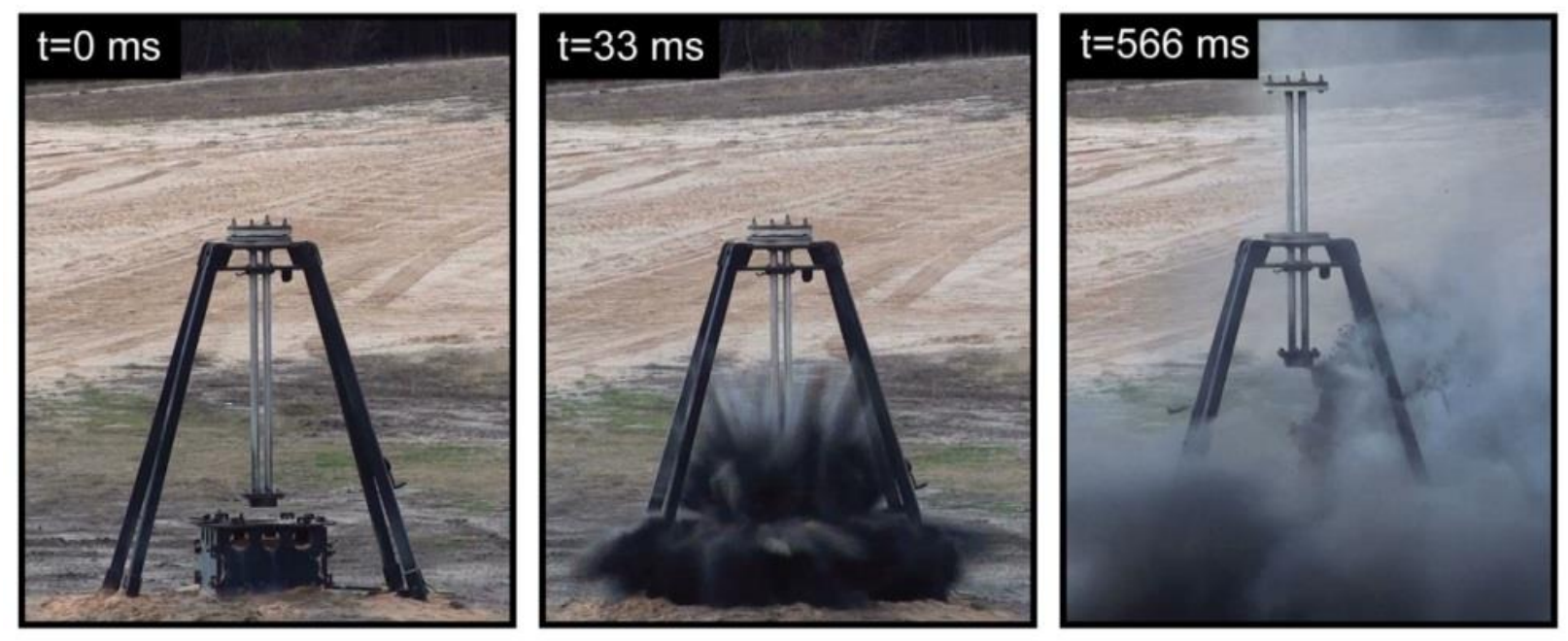

Figure 95: Photographs of the vertical pendulum during a test, illustrating the movement of the vertical pendulum after loading.

Two cameras were routinely used to observe the experiment and measure the pendulum height-time relation. A Phantom V7.1 high speed camera, made by Vision Research, recorded at frames up to 5,000 frames per second (fps) and allowed for high speed imagery of the sand front and pendulum movement. Peak pendulum heights were also easily recorded with a Sony Handycam, recording in high definition (HD) at $30 \mathrm{fps}$ as the pendulum decelerates, stops, and reaccelerates at the apogee allowing over a 0.1 second time span to measure peak height within the resolution of the camera. However, accurate height-time relations are not capable with the Sony. 

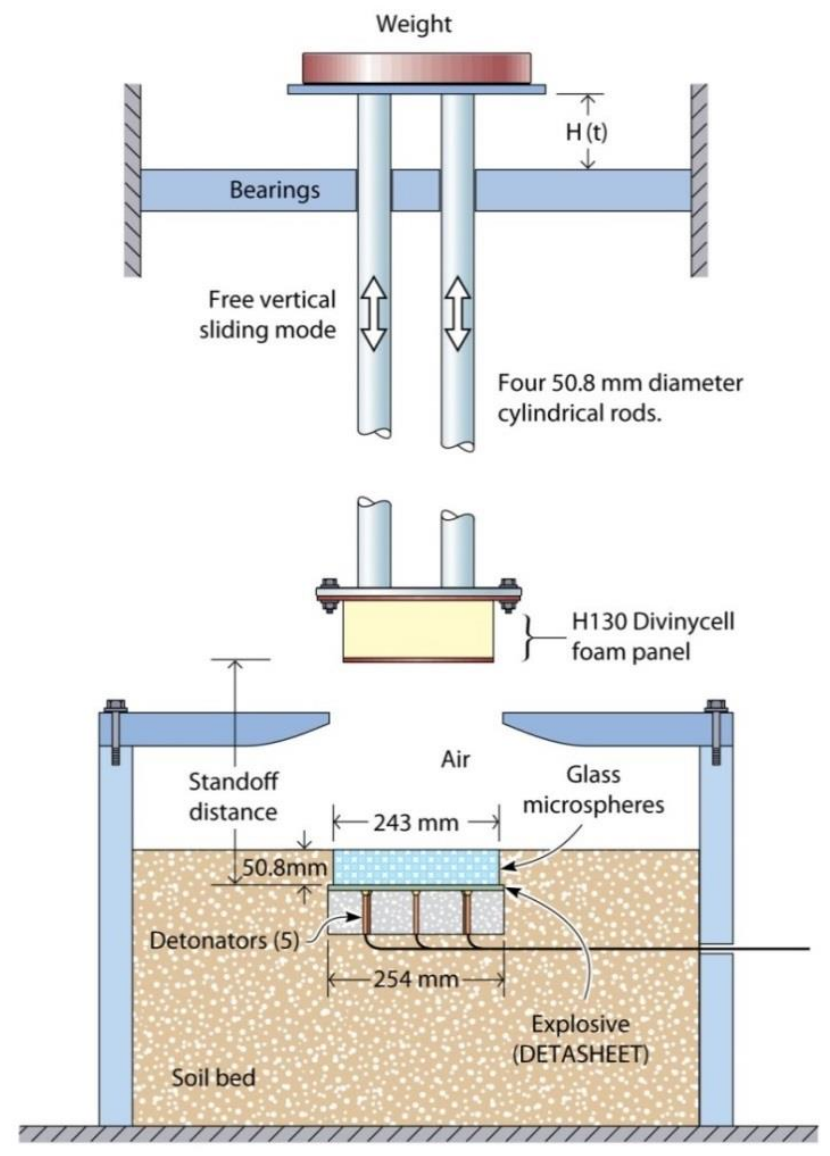

Figure 96: An illustration of the vertical pendulum set-up, showing the relative positioning of the explosive charge assembly, sample, sliding aluminum bar assembly, and soil containment box. The vertical pendulum measures transmitted impulses through the vertical height measurement of a sliding bar assembly after blast loading occurs.

The high velocity impact of the glass microspheres with the sandwich panel samples are classic inelastic collisions. A portion of the kinetic energy from the impacting microspheres is lost in the form of permanent deformation (i.e. energy absorption) within the structures, as is the intent of any energy absorbing material. Conservation of energy theorem, therefore, cannot be successfully used because energies cannot be successful accounted for within the system. Conservation of momentum, however, is valid during an inelastic collision and used frequently to evaluate dynamic loading events. Specifically, impulse measurements are measured to evaluate a structure's effectiveness. 
Impulse is simply the change in the momentum of a system and is equal to the integral of an applied force over a period of time, given by;

$$
I=\Delta j=\int_{t_{i}}^{t_{f}} F(t) d t
$$

where I is the impulse, $\mathrm{j}$ is the momentum, and $\mathrm{F}$ is an applied force. Substituting in Newton's second law of motion into Equation (8.1), the impulse can be reduced to;

$$
I=\Delta j=m\left(v_{f}-v_{o}\right)
$$

where $\mathrm{m}$ is the mass of the entire vertical pendulum assembly plus the sandwich panel sample, $\mathrm{v}_{\mathrm{f}}$ is the final velocity of the rig after the pendulum swing, and $\mathrm{v}_{\mathrm{o}}$ is the initial velocity of the rig after impact occurs.

To determine the impulse of the vertical pendulum, the initial velocity must be calculated (note: the final velocity is zero as the pendulum reaches the maximum height). The initial velocity of the vertical pendulum (i.e. the velocity immediately after sand loading occurs) is calculated from the conservation of energy. After impact occurs, there is no further structure deformation and kinetic energies are converted to potential energies. Precautions are taken to clean and maintain the aluminum sliding bars to minimize contact friction and it is assumed that negligible energy is lost through friction. The conservation of energy simply states;

$$
K E_{o}+P E_{o}=K E_{f}+P E_{f}
$$

where $\mathrm{T}$ is the kinetic energy and $\mathrm{U}$ is the potential energy in reference to initial and final time periods. When initially loaded, no potential energy exists in the pendulum and all energy is 
kinetic. At the peak height, however, all kinetic energy has been converted to potential energy. Therefore the energy balance, Equation (8.3), can be rewritten as the following;

$$
\frac{1}{2} m v_{o}^{2}=m g h_{f}
$$

which can then be used to express the initial velocity of the rig as a function of the maximum pendulum height, $\mathrm{h}_{\mathrm{f}}$. Substituting the initial velocity that can be calculated from Equation (8.4) into the simplified impulse expression, Equation (8.2), and dividing by the cross sectional area, A, of the impacted sample surface, the impulse/area of the entire pendulum assembly can be expressed as;

$$
\frac{I}{A}=\frac{m}{A} \sqrt{2 g h_{f}}
$$

Impulse measurements of the entire vertical pendulum and sample are relatively meaningless by themselves, but the relative impulse mitigation as compared to a rigid, solid aluminum sample provides a measure of effectiveness of the samples. The solid aluminum calibration blocks do not permanently deform and are used as a rigid structure. As a result, calibration tests were performed in conjunction with each sample to provide a comparative data point for the vertical pendulum rig and the ratio of the measured impulses is reported. As sample weights varied, pendulum weights were adjusted so the entire pendulum/sample package exhibited matching weights.

Rear-face pressure measurements on the samples were attempted using a mono-sheet color-developing film. When pressure is applied micro-encapsulated ink ruptures onto the film. Manufactured by Fujifilm, three grades were used in conjunction with each other to provide 
pressure measurement abilities from 10 to $300 \mathrm{MPa}$. The film was sandwiched between aluminum mounting places behind the rear facesheet for the foam-only and corrugated sandwich panels. For the aluminum calibration block the film was placed on the front surface, sandwiched under a thin aluminum front facesheet to provide a clean pressure measurement. The front face pressures and rear face pressures of the aluminum calibration block were assumed to be equal, as all the force was expected to be transmitted through the structure, but validation of this failed to occur. The pressure film set-up is illustrated in Figure 97.

a)

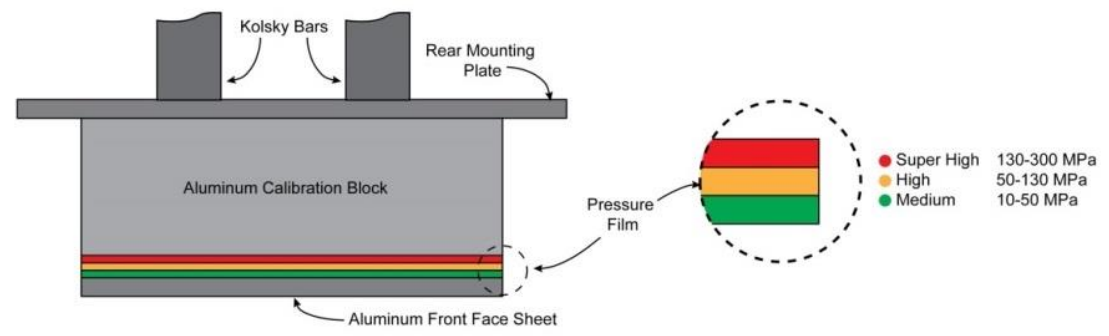

b)

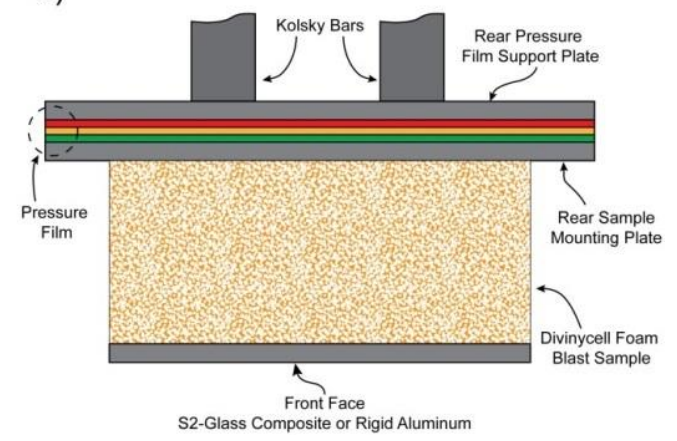

c)

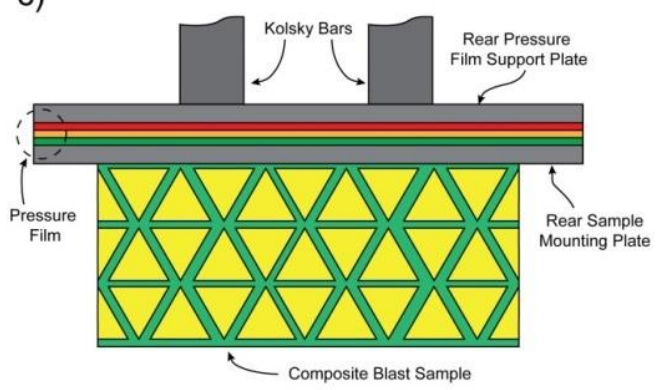

Figure 97: A series of pressure films, designed to change color depending on the applied load, were used to measure sample pressures during vertical pendulum sliding tests.

\section{Vertical Pendulum - Fixed Mode}

An alternate configuration of the vertical pendulum can measure rear-face pressures with a greater degree of accuracy. As illustrated in Figure 98, by rigidly bolting the pendulum fixture to the frame, via the top mounting plates, and instrumenting the aluminum bars with strain 
gauges, the rig is transformed into a parallel measurement Kolsky Bar system. Technically no longer a pendulum, this technique provides strain measurements of the Kolsky Bars that can, in turn, be used to calculate both rear-face pressures and impulses regardless of the fact there is no pendulum movement.

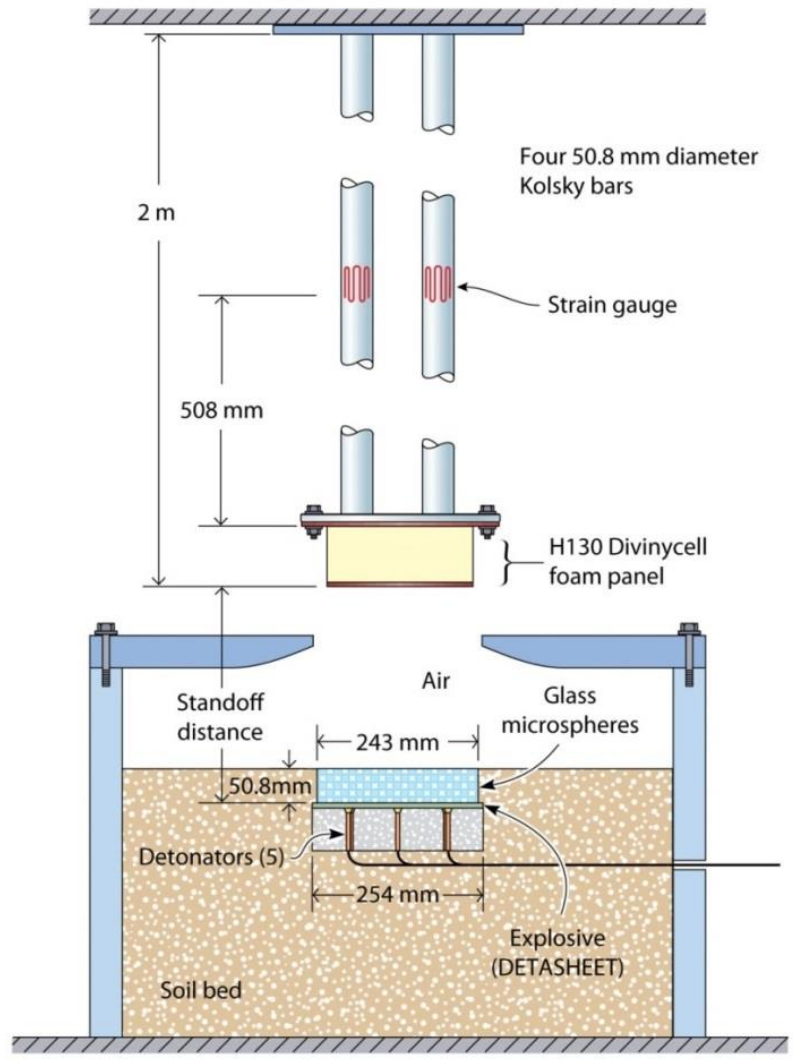

Figure 98: Rigidly fixing the vertical pendulum to the frame allows the aluminum bars to be instrumented with strain gauges and used as Kolsky Bars to measure rear face pressures and impulse of the structure.

Strain gauges are attached to each of the $2 \mathrm{~m}$ long $\mathrm{x} 25.4 \mathrm{~mm}$ diameter aluminum bars at a distance of 10x the diameter in length $(508 \mathrm{~mm})$ from the front most edge of the Kolsky Bars. The strain gauges were expertly adhered to the Kolsky Bars and covered with epoxy to protect them during blast events. During a test event, the strain measurements were recorded at 500,000 $\mathrm{Hz}$ to obtain a detailed strain-time response. The strain measurements were converted to 
determine the stresses of the Kolsky Bars which could then be used to calculate the rear-face stresses observed in the sample.

The Kolsky Bar measurement technique is successful by using the stress waves that develop in a material when dynamically loaded to measure a strain-time relation in the bars. This technique provides an excellent data acquisition method, but is limited by the reflection of the stress wave within the material. The $2 \mathrm{~m}$ long Kolsky Bars provide $598 \mu \mathrm{sec}$ of clean data acquisition before the stress wave reflection reaches the strain gauges. After this time, stress reflections interfere with each other and reliable strain measurements are no longer possible. Figure 99 illustrates the stress wave reflection.

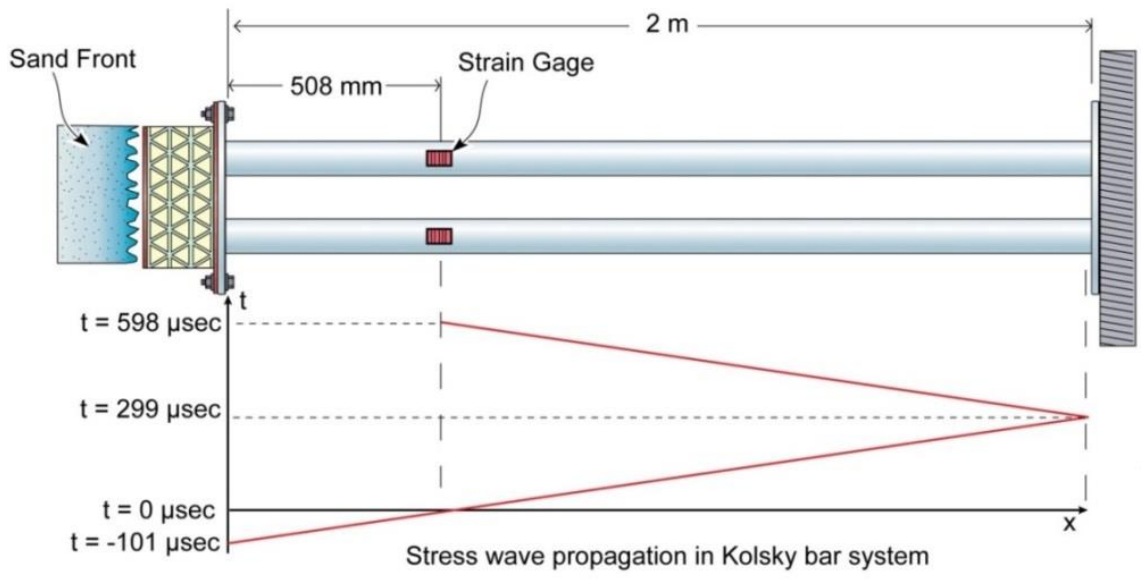

Figure 99: The stress wave developed in the Kolsky Bars transverses through the material providing a clean strain signal until the stress wave reflection returns to the strain gauges, approximately $598 \mu \mathrm{sec}$ after the initial measurement.

\section{Experimental Parameters}

To investigate the sandwich core response under high explosive sand blast loading, several parameters where varied to adjust the transmitted impulse from the glass microspheres to the sample surface. Early experiments (not discussed in this study) showed the greatest energy transfer from the explosive to the soil when the explosive was buried approximately $50.8 \mathrm{~mm}$ in 
depth below fully saturated glass microspheres. This study maintains those parameters for every test in this study and only varies the standoff distance (distance between the explosive and sample front face) and explosive quantity to vary transmitted impulses. The effect of the explosive quantity was evaluated for Detasheet masses of 100, 200, 300, and 400 grams. As shown in Figure 100, the sand front height as a function of time is presented for the explosive charge assembly with only the Detasheet mass varied. These results show a linear response between position and time, yielding a constant velocity of approximately $150 \mathrm{~ms}^{-1}, 200 \mathrm{~ms}^{-1}, 300$ $\mathrm{ms}^{-1}$, and $400 \mathrm{~ms}^{-1}$ for the $100,200,300$, and 400 gram charges, respectively. Characterization of sand front is significant and rarely performed. The development of a planar front was successfully created and provides a constant velocity sand front, important for research focused studies using high explosives driven sand. .
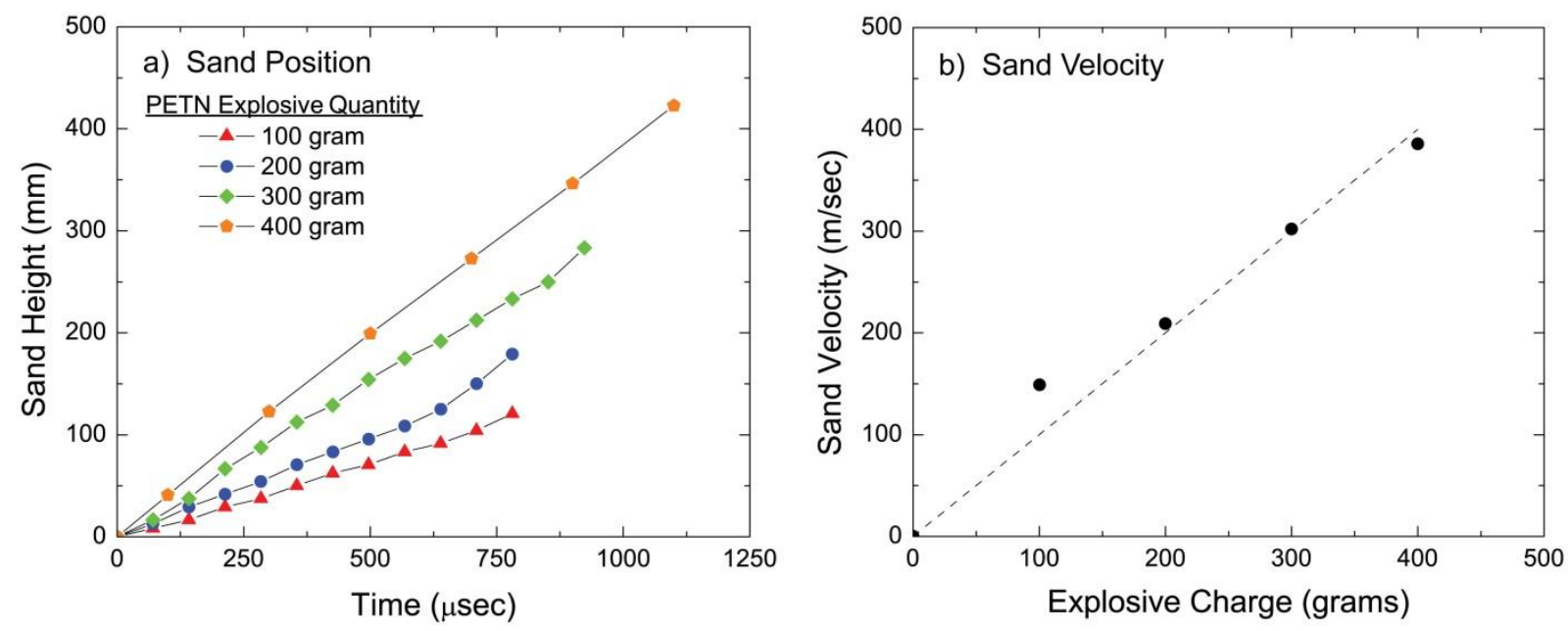

Figure 100: The a) front edge of the high velocity glass microspheres is plotted as function of time, showing a linear response and b) constant velocities based on the explosive charge quantity (relative to the Detasheet only).

The standoff parameter provided a second degree of variability for impulse transfer between the glass microspheres and sample. Based entirely on the sand velocity data shown in Figure 100, standoff would seem irrelevant as the glass microsphere velocities are constant 
regardless of height. However, the effect of microsphere separation (i.e. the change in the saturated microsphere density) occurs over the traveled distance. As seen in Figure 101, the sand front traverses upward to impact the front face of the sample, but varying velocities are present in the microspheres to allow the soil to spread. Knowing this occurs, standoff distances are varied to adjust the transmitted impulses. The standoff distances tested in this study included 14, 19, 24, 29, and $40 \mathrm{~cm}$.
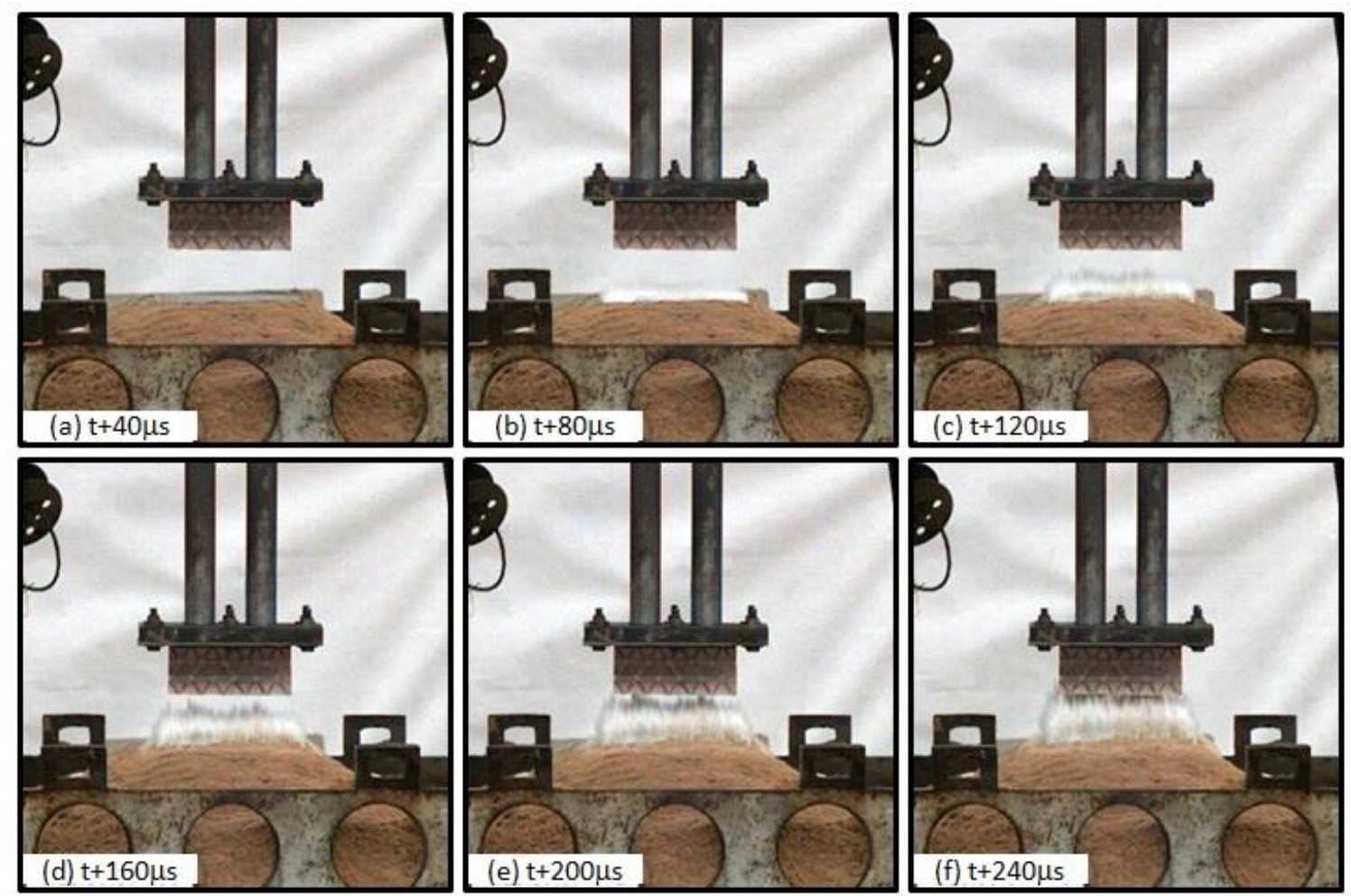

Figure 101: High speed photography captures the sand front of the glass microspheres impacting the hybrid corrugated sandwich structure while the Kolsky Bars measure rearface stresses [134].

\subsubsection{Experimental Results - High Explosive Blast Loading - Multi-Tier}

The failure responses of the hybrid corrugated sandwich structures were identical between both modes of operation of the vertical pendulum rig. Progressive amounts of failure 
were observed in the structures as related to the quasi-static compressive strength. Not surprising, lower strengths cores exhibited failure modes through the entire structure and even core densification. Higher strength cores exhibited a susceptibility to failure at the points closest to impact. Surprisingly, the S2-glass facesheets, chosen because of their high strength until tensile loading, appear to be a predominant failure on the panels. Figure 102 shows a photograph of several post-blast panels tested at a $14 \mathrm{~cm}$ standoff using a 300 gram Detasheet charge including for a) the Divinycell H130 foam-only panel $\left(\sigma_{p l}=3 M P a\right)$, b) open core $\bar{\rho}=15 \%$ panel $\left(\sigma_{p l}=4 M P a\right)$, c) open core $\bar{\rho}=27 \%$ panel $\left(\sigma_{p l}=6 M P a\right)$, and d) foam-filled $\bar{\rho}=27 \%$ panel $\left(\sigma_{p l}=12 M P a\right)$.
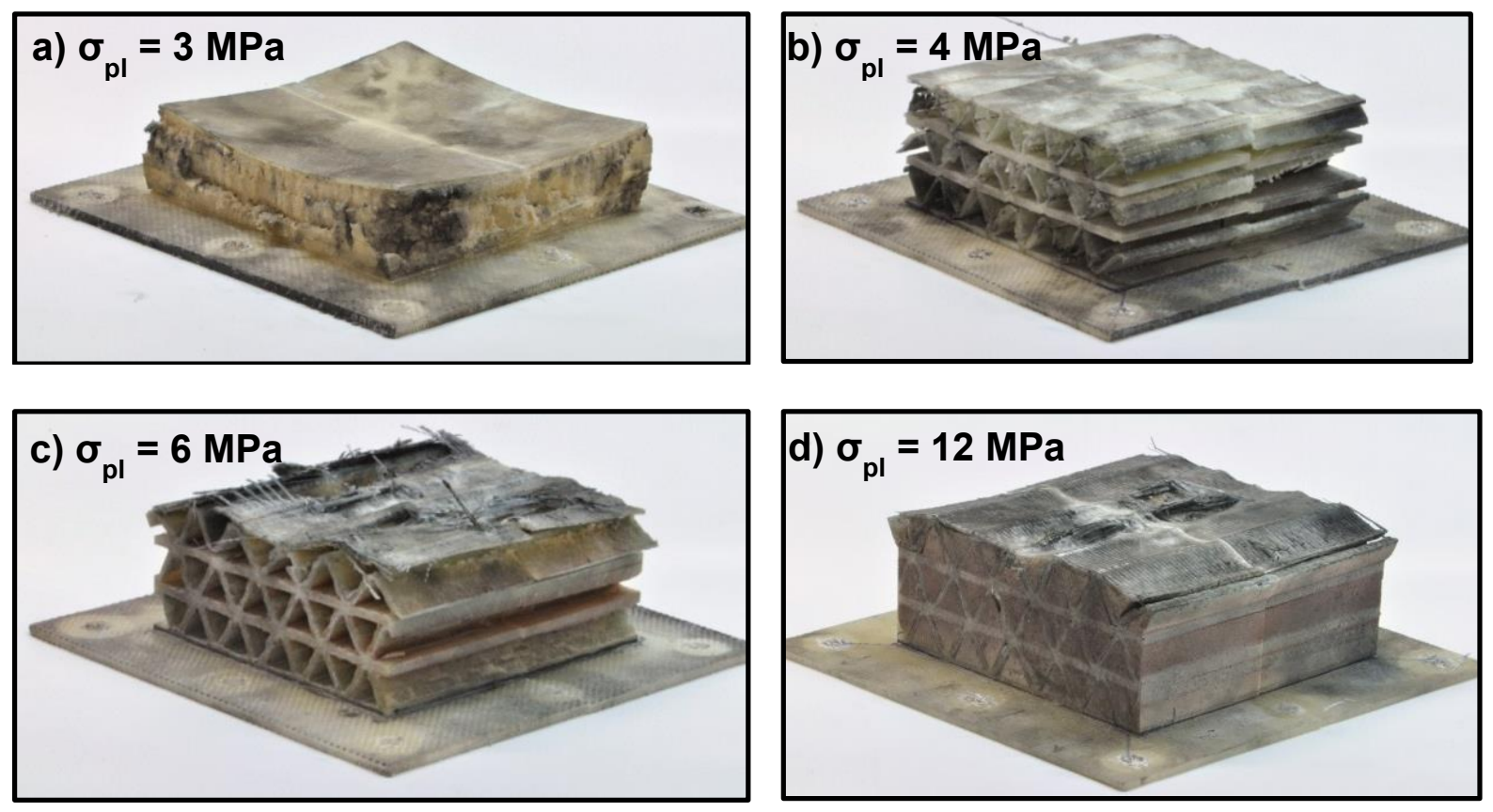

Figure 102: Photographs showing progressive structure damages for a series of samples tested at $14 \mathrm{~cm}$ standoff and 300 gram Detasheet explosive charge for structures with varying quasi-static plateau strengths, $\sigma_{\mathrm{pl}}$, including a) the Divinycell $\mathrm{H130}$ foam-only panel $\left.\left(\sigma_{p l}=3 \mathrm{MPa}\right), \mathbf{b}\right)$ open core $\bar{\rho}=15 \%$ panel $\left(\sigma_{p l}=4 \mathrm{MPa}\right)$, c) open core $\bar{\rho}=27 \%$ panel $\left(\sigma_{p l}=6 M P a\right)$, and d) foam-filled $\bar{\rho}=27 \%$ panel $\left(\sigma_{p l}=12 M P a\right)$. 
Multiple failure modes were observed in the hybrid corrugated structures. Facesheet failure occurred most frequently, failing by tensile yield and shear. The shear failure allowed the glass microspheres to partially enter the core of the panel, a failure response not anticipated. The core appears to fail predominantly by strut microbuckling, and fractured fiber kinking. No instances of Euler buckling could be determined post-mortem. Matrix crazing accompanied all strut failure modes and nodal rotations (the matrix crazing is how to locate a nodal rotation). Shear failure also appears to have developed in localized areas of the strut. Failure is always most predominant in the top (impact side) tier with progressively decreasing levels of failure through the thickness of the structure.

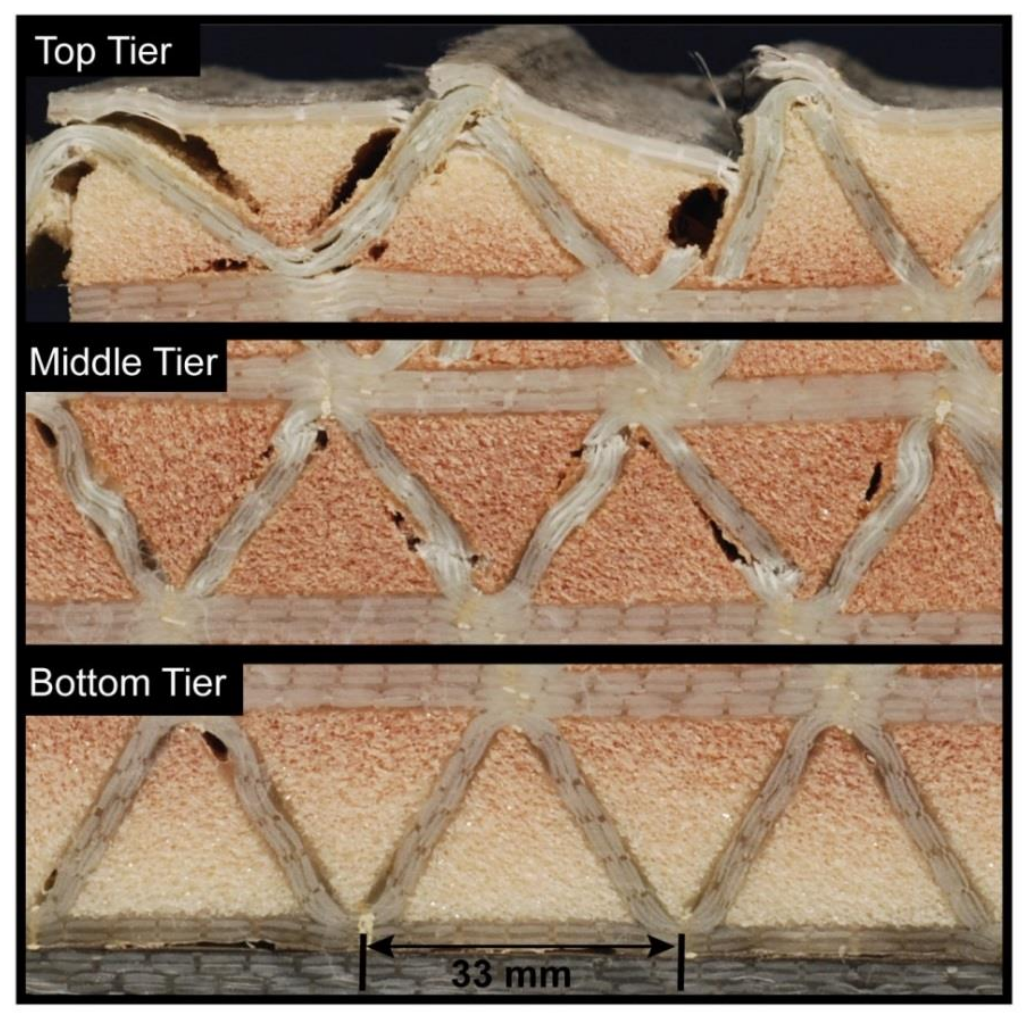

Figure 103: Failure modes observed in the crushing of the hybrid corrugated sandwich panels included strut microbuckling, fractured fiber kinking, facesheet shear, matrix crazing, nodal rotation, and both shear and compressive failure. Failure is always more predominant in the top (impact side) tier with progressive failure through the structure. 
Strictly for comparison purposes, a series of hybrid corrugated sandwich panels is shown in

Figure 104 to show the damage response based on quasi-static compressive strength and increased impulse (through increased explosive charge). The samples shown in Figure 104 are all tested at a $14 \mathrm{~cm}$ standoff distance and use either a 300 or 400 gram Detasheet explosive charge to vary the transmitted impulse.

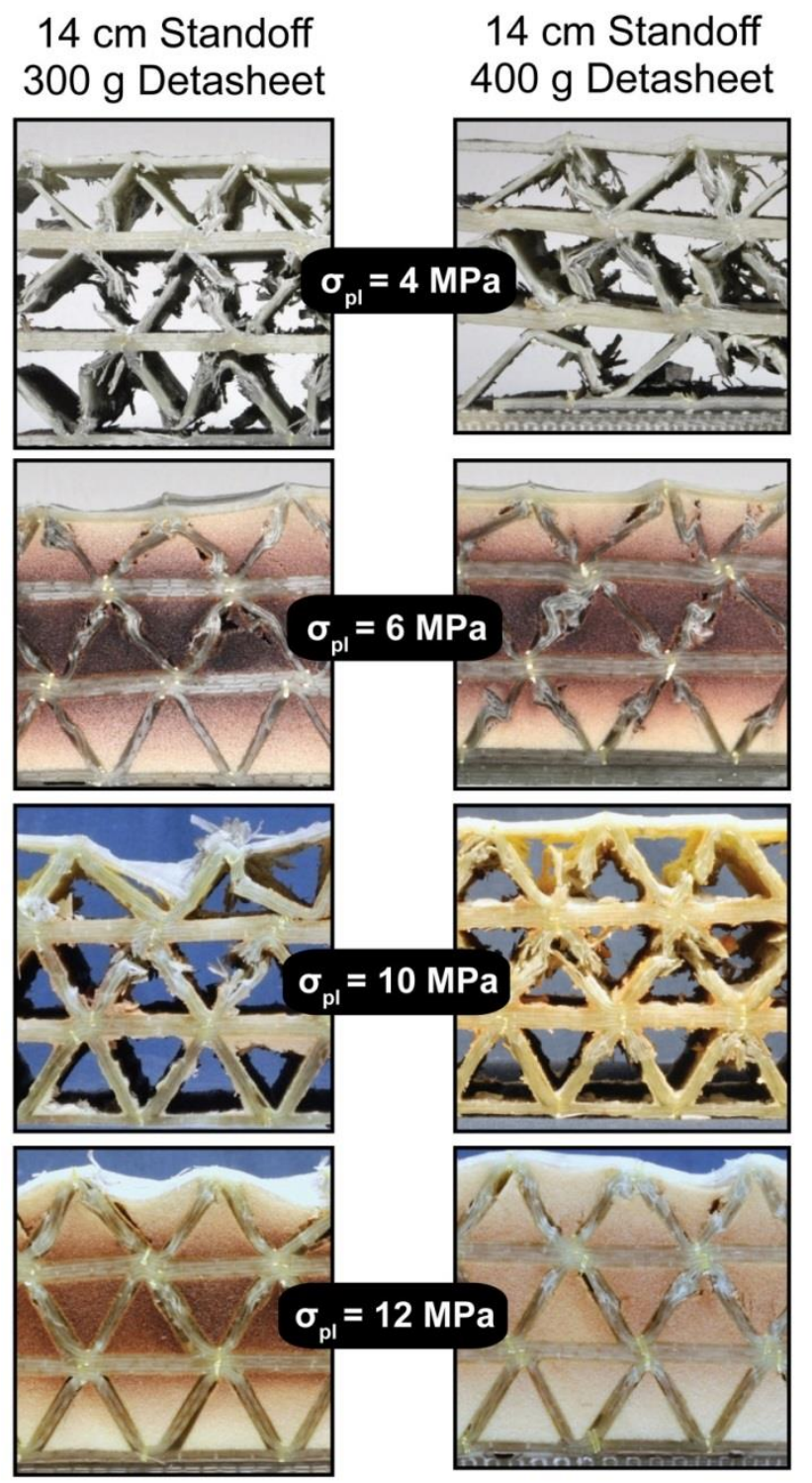

Figure 104: Photograph comparison of hybrid foam-filled and open core structures tested at $14 \mathrm{~cm}$ standoff with either a 300 gram or 400 gram Detasheet charges to illustrate the failure observed in varying core strength panels. Full densification and core failure occurs at the low density cores while only partial core (top tiers) is observed for cores of higher strength. Facesheet failure was observed in all test cases. 


\section{Vertical Pendulum - Sliding Mode}

The vertical pendulum is used to measure a height-time relation of a given mass as related to the applied impulse and blast armor being tested. . Most important is not the single measurement of the height the pendulum when tested with an armor, but rather the variation in pendulum height between a given sample and a solid aluminum rigid block. The solid aluminum rigid block is used as a calibration tool for this rig and provides a method to measure impulse mitigation. The calibration block allows for the majority of energy to be transmitted through the panel into the rig without permanent deformation of the material. The rig can be periodically tested through the use of the calibration block to confirm a repeatable pendulum height movement under a given applied impulse. For every sample panel that is tested, a corresponding calibration block is tested for a reasonable comparison in height given the condition of the rig at that point in time (used to account for cumulative damage from repeated explosive tests). Shown in Figure 105, eight tests are shown to illustrate the pendulum response. A correlation between all tests during their assent upwards can provide indication of unwanted friction or rig bending. Tests that show representative behavior are used to measure the peak height and calculate transmitted impulses. The peak height in each case will differ based on the amount of counterweights used to balance the rig. The maximum travel of the vertical pendulum is $1.5 \mathrm{~m}$, limiting the range of test parameters available. In Figure 105, the rig counterweight is consistent for all tests at $82.8 \mathrm{~kg}$. The results show that the closer the standoff distance, the larger the transmitted impulses. Increased friction or vibrations on the pendulum return do not affect the initial pendulum rise. 

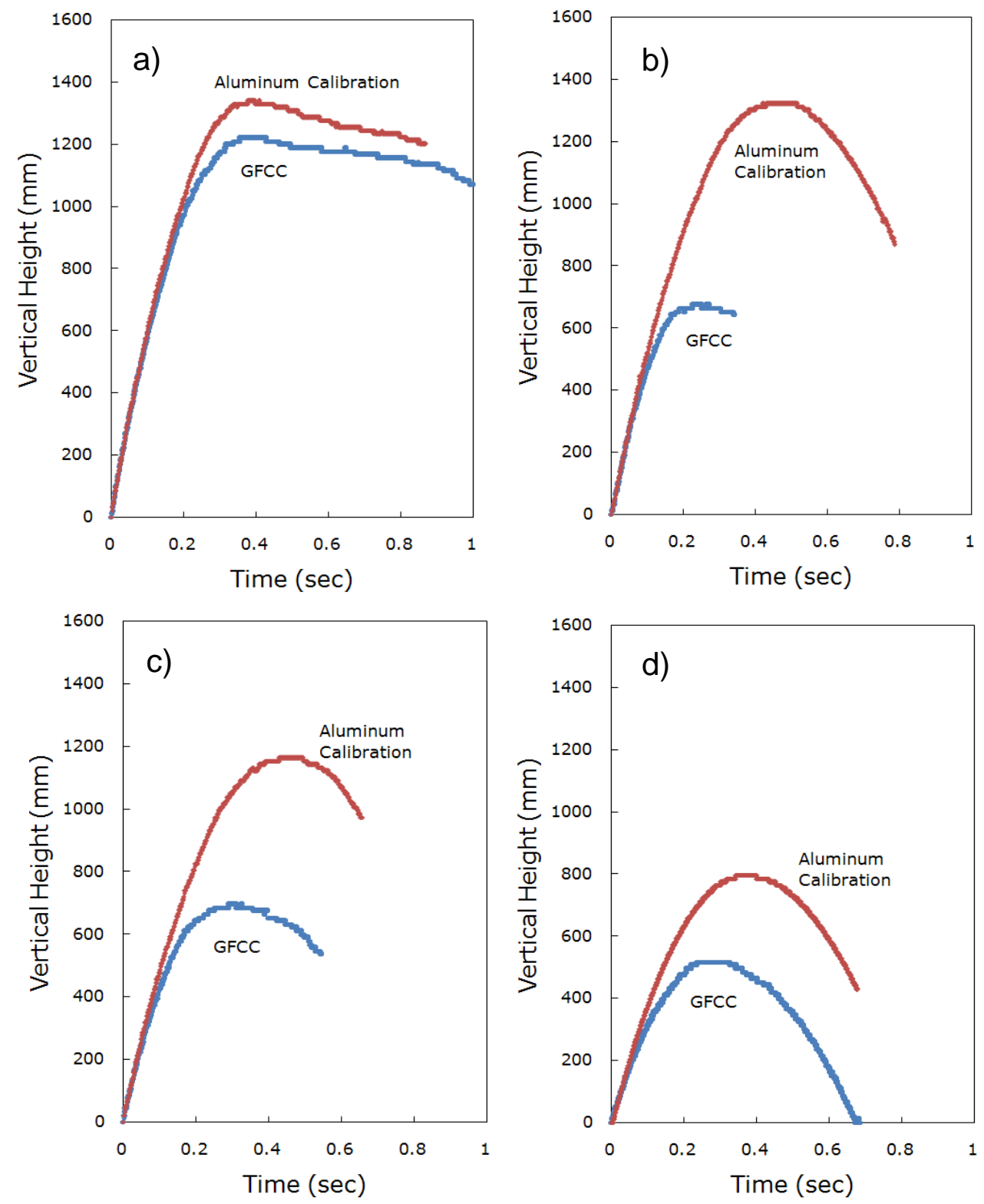

Figure 105: Vertical height displacement comparisons of sliding pendulum for the hybrid glass fiber corrugated core (GFCC), $\sigma_{\mathrm{y}}=12 \mathrm{MPa}$, at standoff distances of a) $\left.14 \mathrm{~cm}, \mathrm{~b}\right) 19 \mathrm{~cm}$, c) $24 \mathrm{~cm}$, and d) $29 \mathrm{~cm}$ standoff distances using a $300 \mathrm{~g}$ Detasheet charge.

The corresponding impulse measurements from the tests shown in Figure 105 are presented in Table 11, illustrating the measured impulse reduction/mitigation that is observed for the three-tier structure of Design 3, $\sigma_{\mathrm{pl}}=12 \mathrm{MPa}$, showing impulse mitigations of up to $30 \%$. At low impulse levels, no impulse mitigation is observed (not shown) and the panel behaviors identical to the calibration block. As applied impulse levels are increased, sample damage is 
observed along with the corresponding impulse mitigation. At high levels of impulse, significant facesheet damage occurs and potentially full core crushing and sample densification (dependent upon the core strength). At high applied impulse levels, the impulse mitigation reduces or even demonstrated an effect of increasing impulse. Increases in impulse are hypothesized to be due the facesheet shape change or facesheet slap.

Table 11: Corresponding impulse measurements and reductions for the vertical pendulum heights for the three-tier corrugated core (Design 3: $\sigma_{\mathrm{pl}}=12 \mathrm{MPa}$ ) and corresponding calibration block along with the corresponding impulse reduction.

\begin{tabular}{|c|c|c|c|}
\hline \multirow[b]{2}{*}{$\begin{array}{l}\text { Standoff } \\
(\mathrm{cm})\end{array}$} & \multicolumn{2}{|c|}{ Impulse (kPa-sec) } & \multirow{2}{*}{$\begin{array}{l}\text { Impulse } \\
\text { Reduction } \\
\quad(\%)\end{array}$} \\
\hline & $\begin{array}{l}\text { Solid Aluminum } \\
\text { Calibration Block }\end{array}$ & $\begin{array}{c}\text { Hybrid Glass Fiber } \\
\text { Corrugated Core (GFCC) } \\
\text { Sandwich Panel }\end{array}$ & \\
\hline 14 & 10.28 & 9.81 & 4.6 \\
\hline 19 & 10.22 & 7.29 & 28.6 \\
\hline 24 & 9.58 & 7.41 & 22.7 \\
\hline 29 & 7.92 & 6.38 & 19.5 \\
\hline
\end{tabular}

\section{Vertical Pendulum - Fixed Mode}

The results from the Kolsky Bar pressure measurement technique, used when the vertical pendulum is placed in a fixed position, provide a method to quantify the pressure transmission mitigation of the panel. A key attribute to a successful vehicle armor is the ability to minimize the transmitted pressures which reduces the peak loads on critical vehicle structural elements. Representative pressure-time responses are shown in Figure 106, for the rigid aluminum calibration block (a), cores with quasi-static yield strengths of $12 \mathrm{MPa}(\mathrm{b}), 4 \mathrm{MPa}$ (c), the Divinycell H130 foam core with a strength of $3 \mathrm{MPa}(\mathrm{d})$ for samples tested at a $14 \mathrm{~cm}$ standoff 
and using a 300 gram Detasheet explosive charge. An indication of the ability for through structure pressure reduction is provided by the initial slope of the pressure-time response. As seen with the calibration block, an immediate spike in pressure is observed, indicating the structure is transmitting the applied pressures through the sample with little energy absorption. The foam-filled corrugated core, Figure 106 (b), has a reduced pressure-time response indicative of energy absorption of the core with a successfully reduction in the peak pressure transmitted. The open core structure, Figure 106 (c), provides improved energy absorption with the lowest peak strength observed at $23 \mathrm{MPa}$, nearly a 30\% reduction from the calibration block. Finally, the Divinycell H130 sample, Figure 106 (d), initially shows the lowest slope in the pressure-time response, most ideal for pressure reduction, but reaches full densification while the structure is still being loaded and then alters the pressure-time response to a similar behavior as the solid aluminum calibration block. The loading being applied after densification was sufficient enough to raise the pressures to be equivalent to the solid calibration block and this structure loses its effectiveness as an armor. 

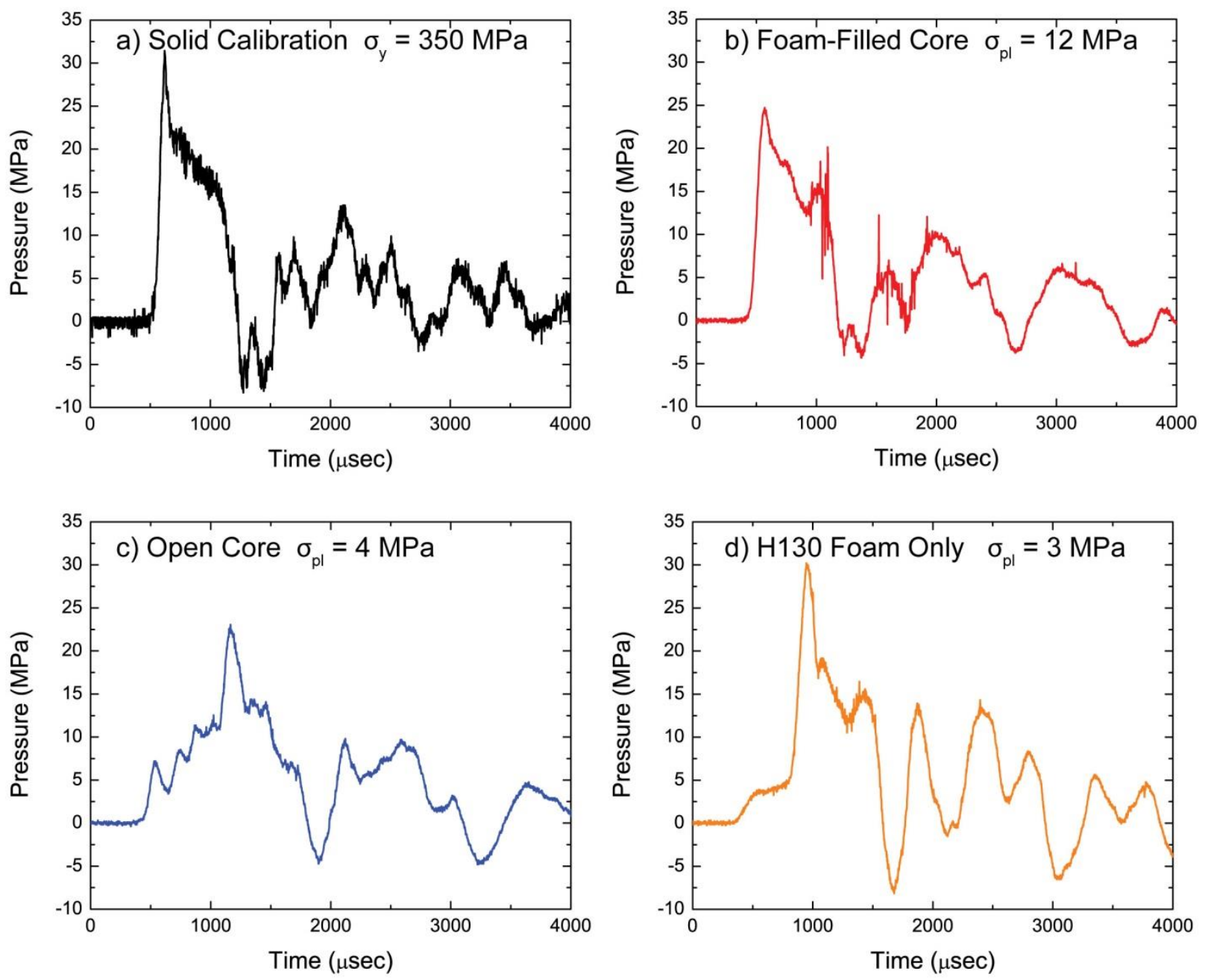

Figure 106: Pressure-time response obtained from the Kolsky Bar measurement technique tested at a $14 \mathrm{~cm}$ standoff with a $300 \mathrm{~g}$ Detasheet charge for a) the solid aluminum calibration block, b) foam-filled core, c) open core, and d) Divinycell H130 foam only samples.

\section{Comparative Results - Foam Analysis}

Ideally, the pressure and impulse measurement made from both methods of operation of the vertical pendulums rig should be in agreement. An analysis using the foam-only panels was conducted to evaluate the results of the experiment. Most important was the response of the panels under sand loading. Figure 107 shows a comparison for tested samples in both the fixed and sliding modes of the vertical pendulum. The results, albeit limited, showed near identical 
responses in core crushing and densification. An analysis of the peak and permanent strains showed less than a 5\% difference between core strains, an acceptable tolerance for this experiment.

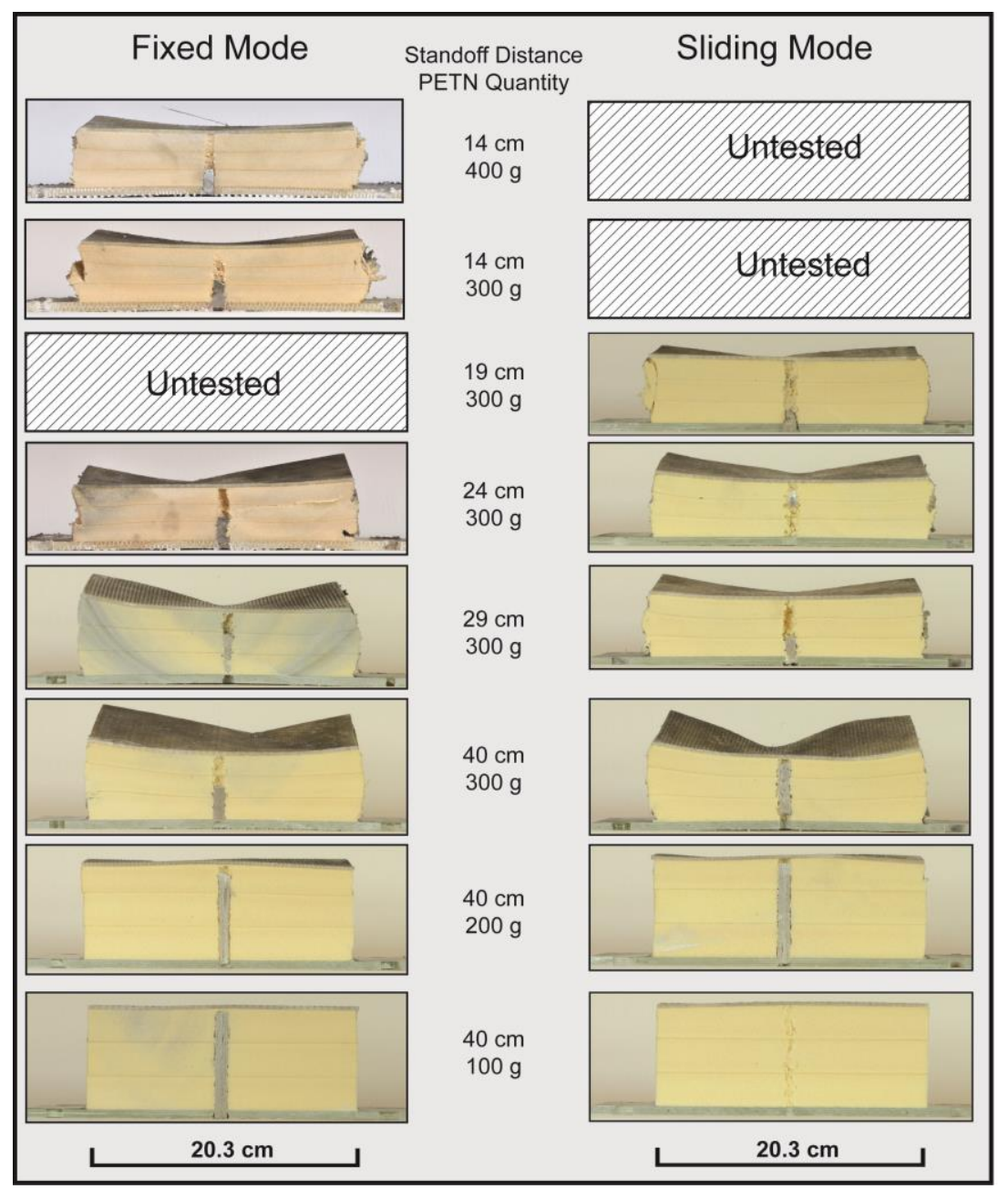

Figure 107: Deformed Divinycell H130 foam samples with composite (non-rigid) front facesheet after being tested at different standoff and explosive charges (i.e. different applied impulse levels).

The comparison between the measured pressures take from the rear-face pressure film used in the sliding mode and the Kolsky Bars in the fixed mode showed significant disagreement. There was an approximate $4 \mathrm{x}$ increase in pressures measured with the pressure 
film over the Kolsky Bars, but a consistent linear relation between the pressures was observed, Figure 108. Modeling efforts by Holloman [128] where used to predict the pressure-time response on this identical Kolsky Bar system and results predicted pressures close to the Kolsky Bar measurements. Three grades of pressure film where used in series to provide a wide pressure range coverage, ranging from 10 to $300 \mathrm{MPa}$. If a lower rated pressure film exceeded the limit then the localize over-limit pressure area was measured by the next higher pressure response film. However, under the high impulse test conditions, localized areas of even the maximum pressure film (300 MPa) exceeded its capability, as seen in Figure 109(d). The pressure film data provided an excellent method to assess load distribution that the Kolsky Bars are unable to provide. As a result of the susceptibility of the pressure film to incomplete data, and limited data, combined with prior successful research with this Kolsky Bar measurement technique, the Kolsky Bar data was chosen to be used for further analysis.

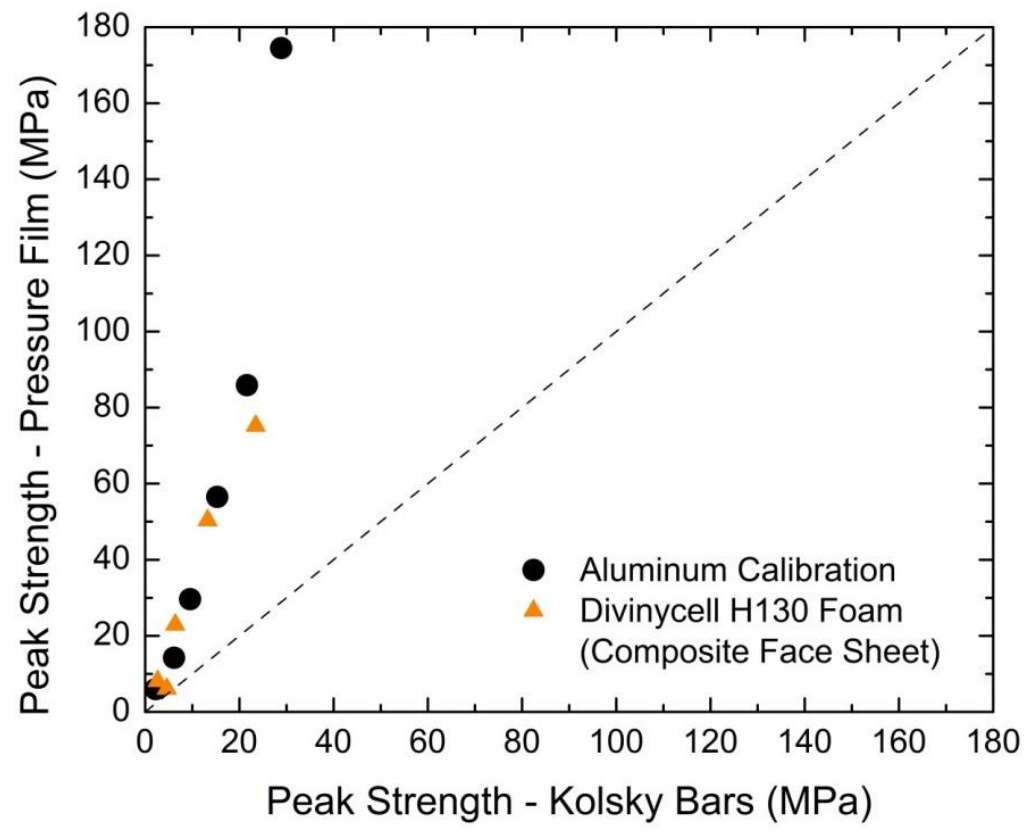

Figure 108: Comparison of peak pressure measurements from the pressure film and Kolsky Bar methods show nearly a 4x difference for all impulse levels. 


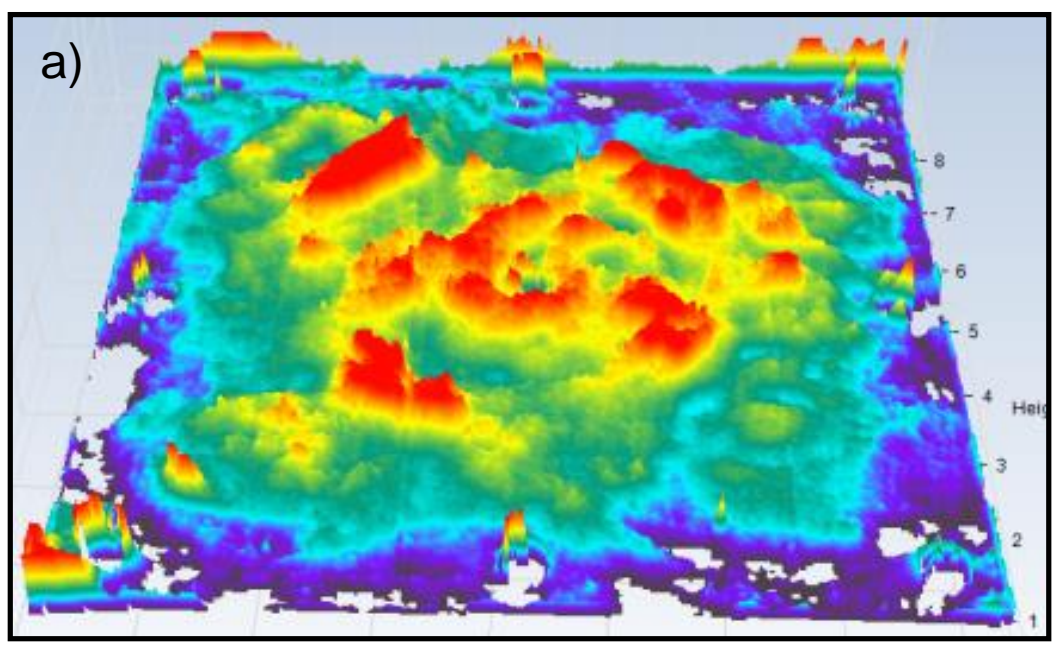

a)

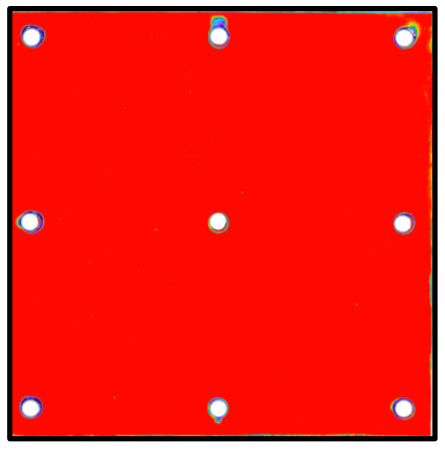

b)

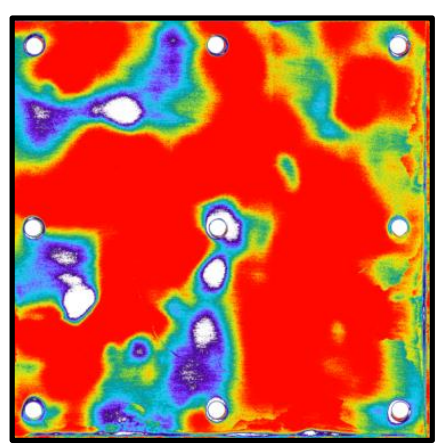

c)

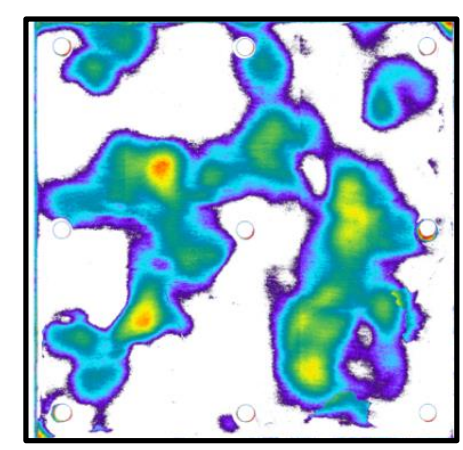

Figure 109: The pressure film provided a method to analyze both the rear-face and frontface pressure distribution. a) The rear-face pressure measurement shows localized pressures in the center of the core. The front-face pressure on the solid calibration block shows the pressure distribution using the b) medium grade (10-50 MPa), c) high grade (50$130 \mathrm{MPa})$, and d) super-high grade (130-300 MPa) pressure films.

\section{Pressure-Impulse Response}

Rear-face pressure measurements where obtained for the hybrid cores (both foam filled and open) through the use of the Kolsky Bar measurement technique. Pressure measurements ranged 2 to $38 \mathrm{MPa}$ for all of the samples and exhibited a critical pressure reduction regime for the each structure. As shown in Figure 110, when initially loaded at low impulses, each core acts similar to the rigid, aluminum calibration block and no permanent deformation occurs. Equally, there is no pressure reduction from the panel at these low impulse levels. As the applied impulse 
levels increase, the panels begin to exhibit damage and permanent deformations. During the impulse loading ranges prior to observed densification, rear-face pressures drop by amounts up to $30-40 \%$. Once impulse levels are increased sufficiently, densification occurs and facesheet slap is observed. Facesheet "slap" is a term used to define full densification of the structure at speeds where the front facesheet is sufficiently accelerated and rapid decelerated by the fully dense structure causing a potential increase in loading. Full densification was observed in only the Divinycell H130 samples due to experimental limitations on the explosive limits that the vertical pendulum can survive. These results show the specific capability to reduce the throughthickness pressure, a key requirement in vehicle armor. Insufficient data exists to draw a conclusive relation between the foam-filled and open core structures. However, both structures so pressure reduction capability up to $40 \%$. The results warrant the future study of this panel at higher impulse levels, unable to be performed with this experimental set-up.

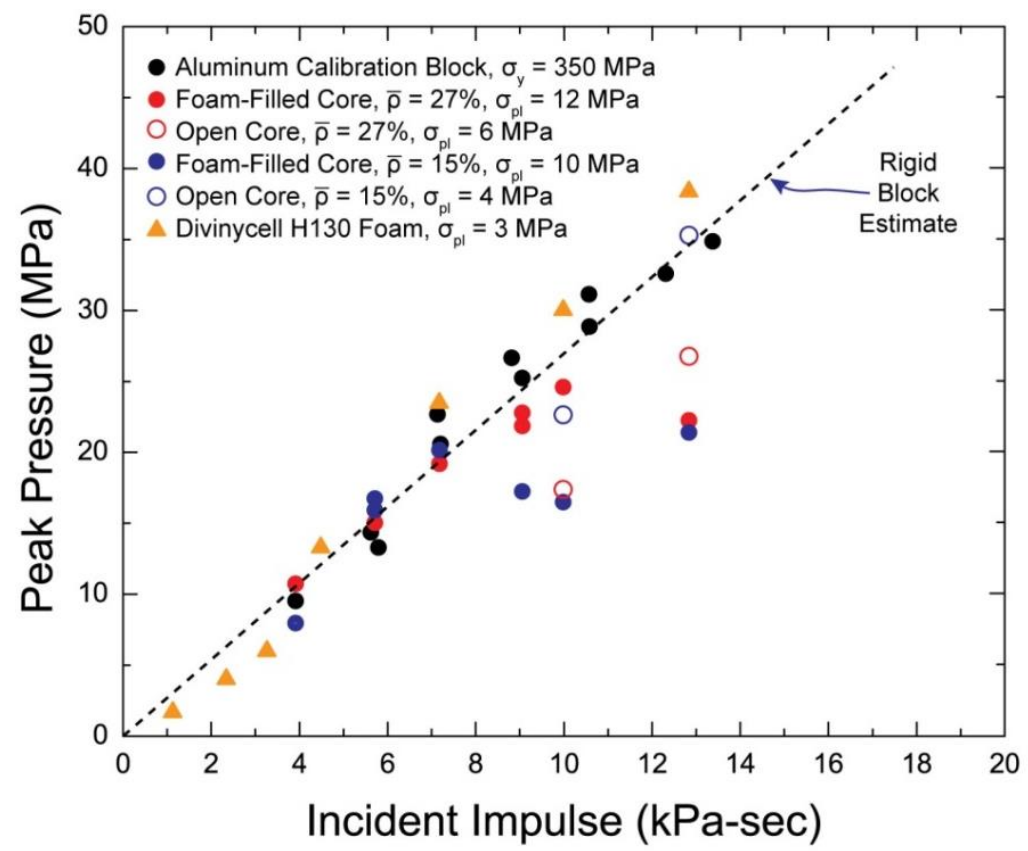

Figure 110: Peak pressures measured by the Kolsky Bars on the vertical pendulum while in fixed mode shows rear-face pressure reductions until full densification is reached during dynamic crushing, at which time pressure spikes are observed. 
Impulse levels could be measured with the Kolsky Bar pressure data by integrating the pressure measurement over the elapsed time. Clean pressure measurements, as seen in Figure 106, are limited by the interference of the stress waves in the Kolsky Bars. The first stress wave reflection is observed approximately $598 \mu \mathrm{sec}$ after initial strain occurs at the strain gauge position. Impulse measurements can only be accurately measured during the clean measurement time limit, as illustrated in Figure 111.

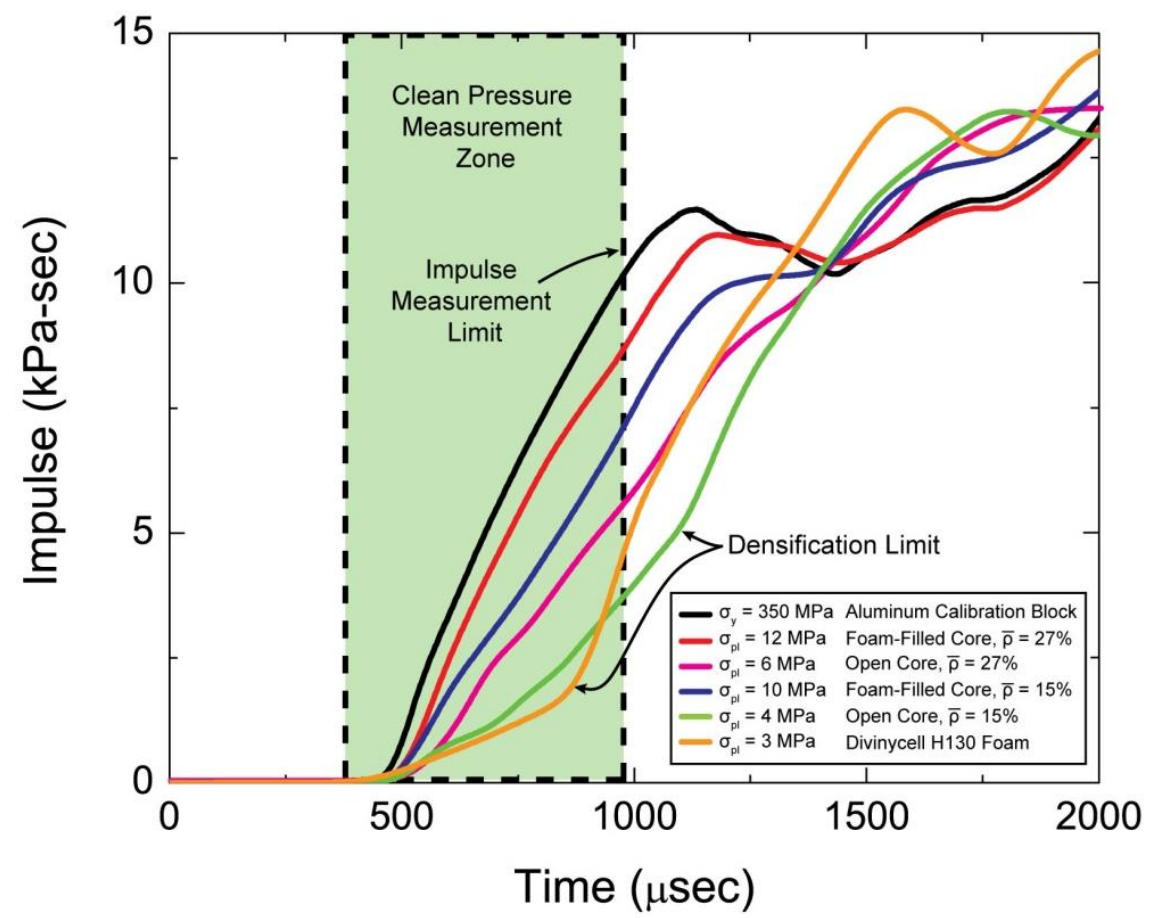

Figure 111: Impulse measurements can be measured in the Kolsky Bar pressure-time response, but clean measurements can only be made before the stress wave reflection in the material returns to the strain gauge position. Results shown are for a $14 \mathrm{~cm}$ standoff and 300g Detasheet charge.

The impulse values normalized with the calibration block response are measured up to the shock wave reflection limit and plotted for comparison in Figure 112 as a function of a normalized strength of the quasi-static core plateau strength, $\sigma_{\mathrm{pl}}$, by the incident peak dynamic pressure of the calibration block as measured by the Kolsky Bars, $\mathrm{P}_{\text {incident }}$. Within this regime, all 
samples show a response either equivalent to the rigid block or exhibit crushing. The crushing of the structure allows energy to be absorbed from the system and transmitted impulse levels are reduced. Impulse levels ranged from 30-90\% of the calibration block. The impulse-time response reveals samples that have reached their densification strain, which is identified by a drastic change in slope, as shown in Figure 111 for the panels with low plateau strength (Divinycell foam and the open core with $15 \%$ relative density). These results can be used as a design tool for future optimization. With a sufficiently thick sample, impulse mitigation could be increased for the cores with low plateau strength. For this experiment, the sand loading persists to approximately $1200 \mu \mathrm{sec}$, shown as the point of inflection on the impulse-time curves in Figure 111. A structure with sufficient energy absorbing ability and that does not fully densify (and exhibit slap) is the ideal structure. Further, the slope of the impulse-time response will provide an average pressure applied to the panel. While this does not indicate the peak pressures transmitted through the structures, it does indicate the pressure reductions that are possible over time. Once the initial shockwave reflection rebounds to the position of the Kolsky Bar gauges, no further conclusive results cannot be drawn on the measured impulse. 


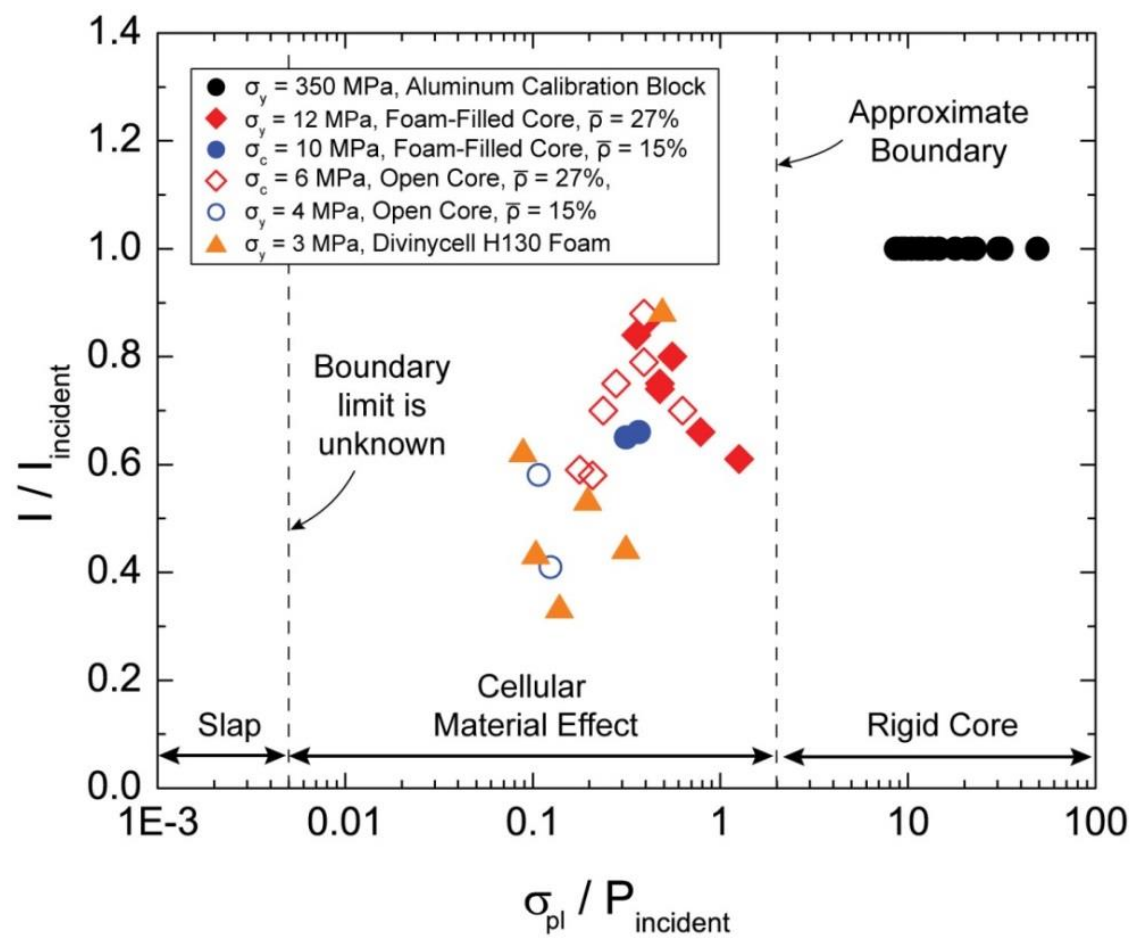

Figure 112: Normalized impulse levels for the sandwich structure cores as measured through use of the pressure measurements from the Kolsky Bars, plotted against the normalized quasi-static core strength over the incident dynamic pressure of the calibration block as measured by the Kolsky Bars. All samples shown have an S2-glass composite facesheet.

Through use of the vertical pendulum, while in sliding mode, full impulse measurements are obtained as described earlier through peak pendulum height measurements. The impulse measurements provided a mixed response of panel performance and illuminated a potential facesheet effect. Plotted in Figure 113, impulse data is normalized with the rigid calibration block, plotted as a function of normalized strength. The samples exhibiting full densification entered the slap regime, and an approximate boundary could be established to predict this transition. When the samples slapped, impulse values were generally observed to be greater than the calibration block by up to a $10 \%$ increase in impulse. Between the rigid core response and slap response, a regime of impulse mitigation was observed by the cellular structures/materials. Corrugated sandwich panels in this region responded with an impulse mitigation up to $35 \%$ of 
the rigid calibration block. The Divinycell foam, however, appeared not to provide any impulse mitigation even though densification/slap did not occur. In fact, increases in impulse were observed for the Divinycell foam samples. Evaluation of all the foam-only structures indicated a significant deformation of the facesheet into a concave shape, Figure 107. It is hypothesized that the facesheet deformation and shape change results in the redirection of the soil ejecta in part back towards the incoming source, thereby increasing transmitted impulses. The hybrid core samples also exhibited localized facesheet failure, but did not exhibit the macroscopic concave deformation. For this reason, it is believe the hybrid corrugated structures were more immune to the facesheet effects. The $30 \%$ impulse mitigation that the hybrid corrugated structure can provide shows significant potential for use as a vehicle armor technology.

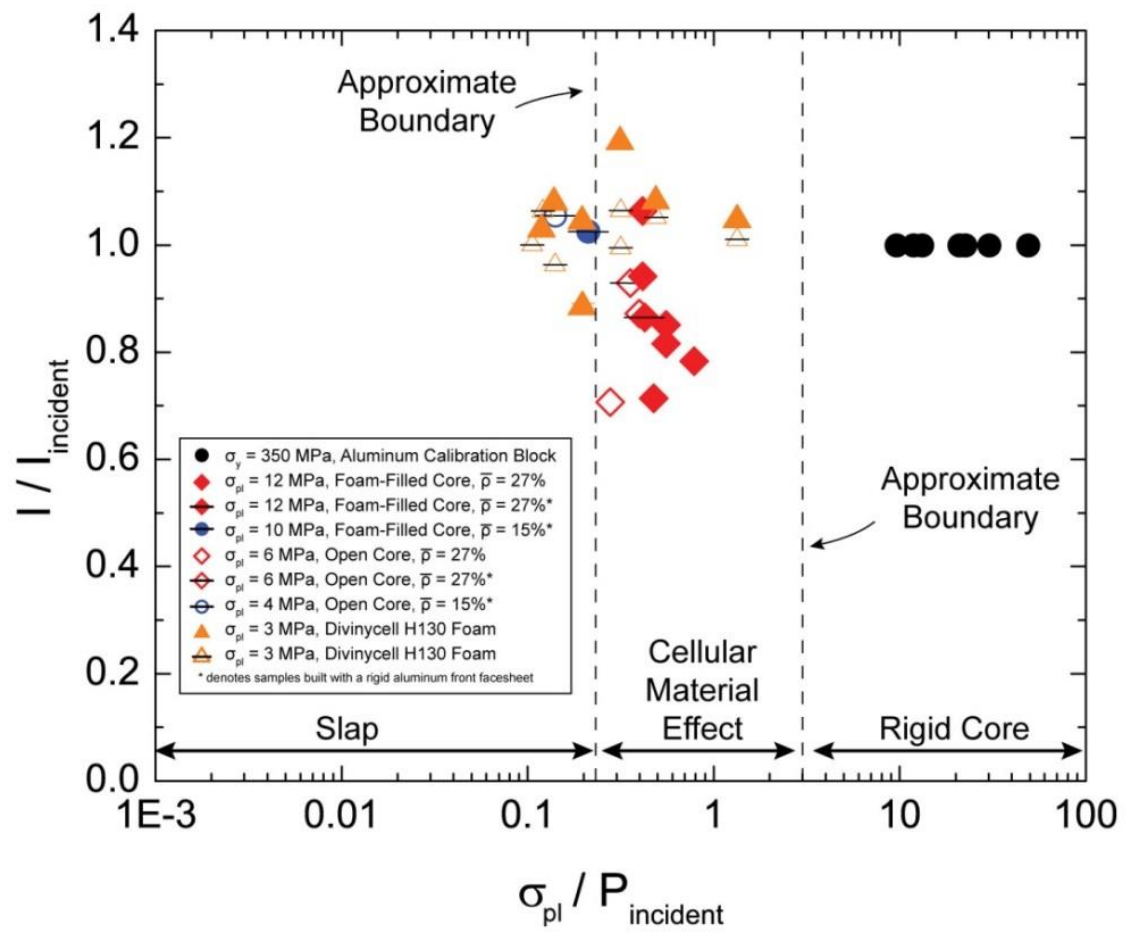

Figure 113: Normalized impulse levels for the sandwich structure cores as measured by the vertical pendulum (sliding mode) and plotted against the normalized quasi-static core strength over the incident dynamic pressure of the calibration block as measured by the Kolsky Bars. Several samples with rigid facesheets were tested to investigate the facesheet effect and are included in this figure and denoted as such. 


\section{Rigid Front Facesheet Effect}

All the cores tested that exhibited damage also exhibited facesheet failure. The hybrid corrugated structures exhibited localized failure between nodes, Figure 103and Figure 104, while the Divinycell H130 foam panels, Figure 107, responds with a macroscopic concave shape. Due to the consistent failure of the facesheet, the overall responses presented above, are indicative of the entire structure response. To isolate the response of the core and remove the effect of the facesheet deformation and shape design, a rigid aluminum front facesheet was investigated for a small number of samples. Shape designs are an important factor to high explosive sand blast loading as they can significantly affect the collision dynamics and total impulse applied to a structure. As illustrated in Figure 115, with the assumption that the impacting soil does not adhere to the sample surface, the approximate transmitted impulse, $\mathrm{I}_{\mathrm{t}}$, relation to a rigid plate shape is given. A rigid plate, Figure 115(b) deflects the impacting soil in a parallel direction with the impact surface and the transmitted impulse due to shape design is equal to the original impulse, $\mathrm{I}_{0}$. If however, a concave shape is formed Figure 115(c), the impacting soil is deflected in a direction towards the original starting position and increases the transmitted impulse greater than the original impulse, $I_{t}>I_{0}$, Successful armor vehicles, such as the Cougar MRAP, use a Vhull design to deflect incoming soil impacts. This shape design has the effect of reducing the transmitted impulse below the original impulse, $\mathrm{I}_{\mathrm{t}}<\mathrm{I}_{\mathrm{o}}$. 
a) Initial geometry

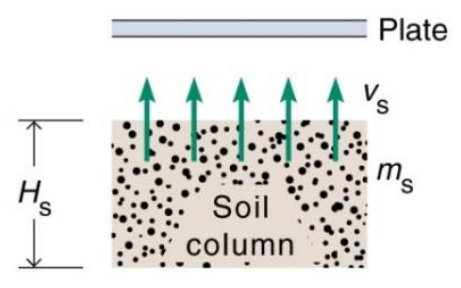

c) Concave deformed

$\left(I_{\mathrm{T}} \gg I_{0}\right)$

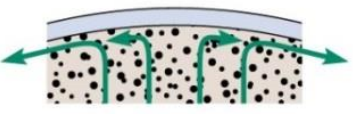

b) Rigid plate $\left(I_{\mathrm{T}}=I_{0}\right)$

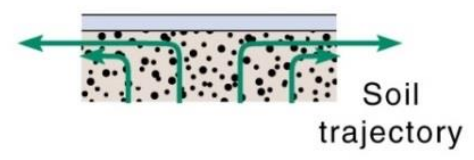

d) Convex deformed

$\left(I_{\mathrm{T}} \ll I_{0}\right)$

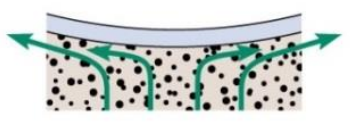

Figure 114: The shape design can affect the transmitted impulse of dynamic soil loading on a structure.

Localized failure of the front-face composite facesheet also failed in a similar manner, creating a concave shape between nodal positions that could either catch or defect back impacting soil. While shape designs are important to the overall response of an armor technology, for the purely compressive response of the core, the effects of shape design complicate the analysis of the core. To investigate the effects of shape design, a rigid front facesheet made from $12.7 \mathrm{~mm}$ thick aluminum replaced the composite sheet on several Divinycell H130 foam samples. Investigating the impulse mitigation of the core with a rigid front-face, the samples were testing on the vertical pendulum in sliding mode. The result yielded an improved response from the Divinycell H130 foam panels. As shown in Figure 115, the concave deflections of the Divinycell H130 foam panels were reduced and an improved out-ofplane compressive loading could be imparted on the sample. The impulse measurements of the rigid facesheet samples, Figure 113, showed a reduction in transmitted impulse from non-rigid front facesheet samples. 


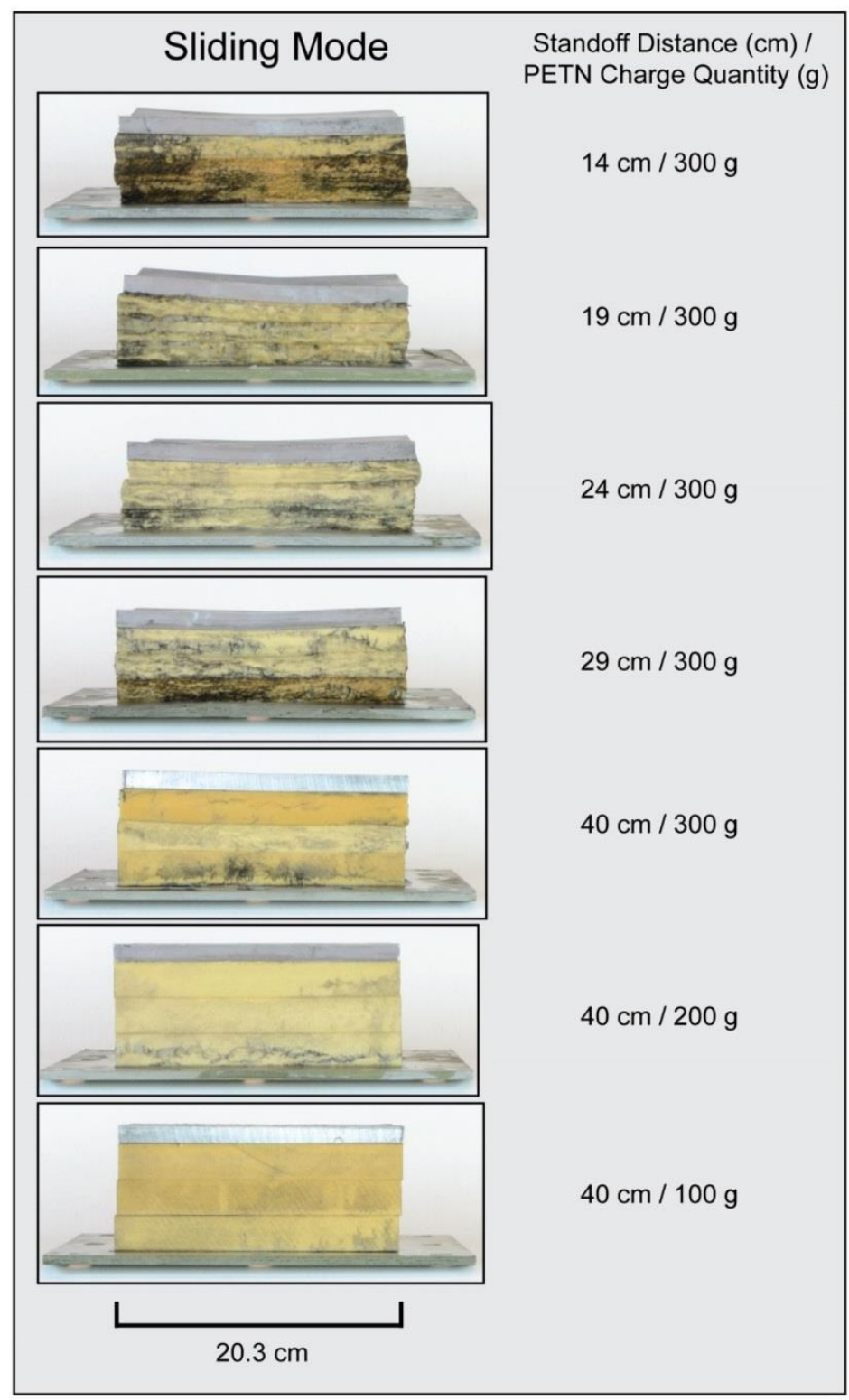

Figure 115: Divinycell H130 foam samples with a $12.7 \mathrm{~mm}$ aluminum (rigid) front facesheet were tested at different standoff distances and explosive charges to vary the applied impulse level. These samples showed a reduced front face deformation by the removal of the concave deformation observed with the composite front facesheet.

The rigid facesheet Divinycell H130 foam-only samples provided a unique opportunity to measure both the peak and permanent strains for high explosive sand blast loading. A nickelfoam core could be added to the center of the Divinycell H130 foam to capture peak and permanent strains, a technique that was not possible with the corrugated cores. Permanent strain 
was measured through the use of a nickel-foam cylindrical core inserted into a cylindrical cavity drilled into the center of the sample through the back face up to the inner edge of the aluminum front facesheet. The nickel-foam was manufactured by Novamet and exhibits less than a $1 \%$ strain recovery and a compressive strength of $0.5 \mathrm{MPa}$, making it an ideal material to measure peak strain with the Divinycell H130 foam panels. After testing, the sample was section cut and the height of the core was measured to determine peak strain. Similarly the permanent deformation of the core in the center was also measured. As shown in Figure 116, the peak strains of the samples with rigid front faces are equivalent to the flexible front faces, and given that the structure has reached full densification during the higher impulse levels, a plateau strain is anticipated. Interestingly, the permanent strain is about half of the peak strain and indicates the foam has a significant spring-back recovery. Spring-back effects can cause increases in impulse similar to shape designs by "throwing" the soil ejecta back in the direction it came to increase the transmitted impulse. This effect would be observed for quick response spring-back and provides a hypothesis for the increased impulses observed for Divinycell foam, a significant parameter that should be investigated in future studies. 


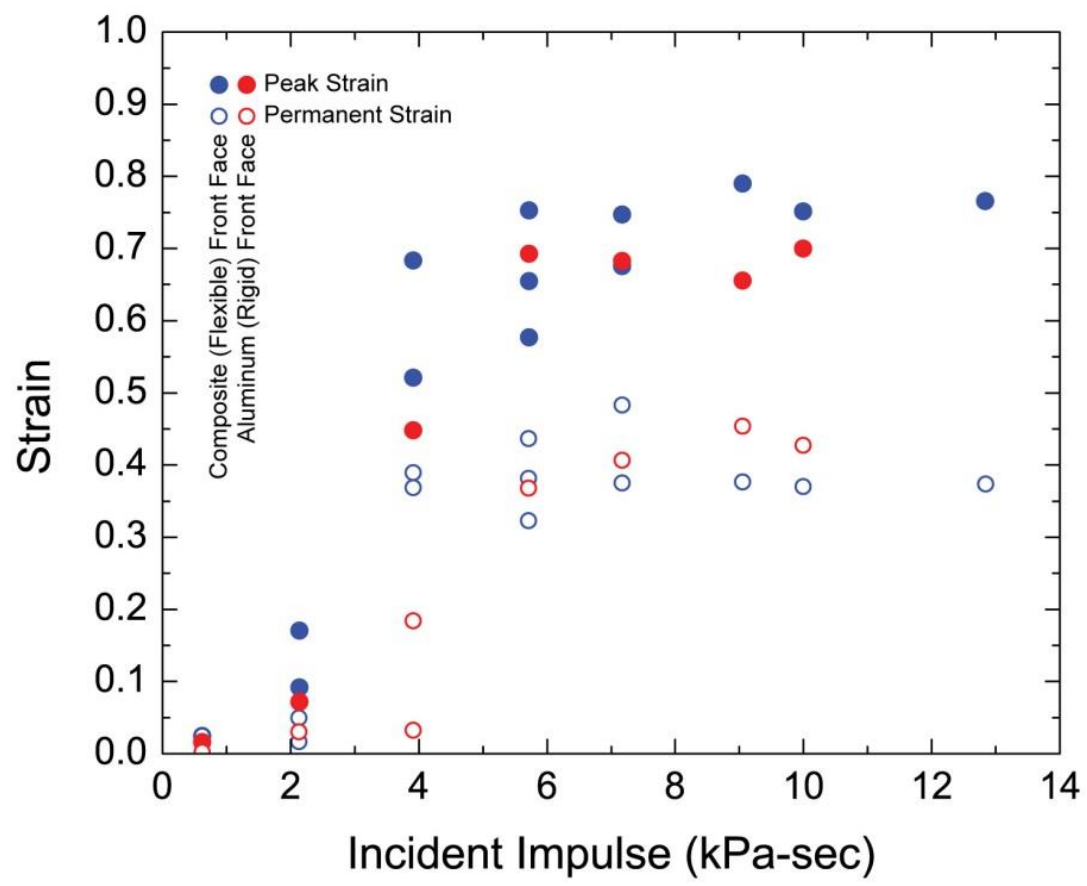

Figure 116: Peak and permanent strains measured for the Divinycell H130 foam. A nickel-foam core inside of the sample was used to measure peak strains.

A limited number of hybrid corrugated samples were manufactured and tested with the rigid front facesheets to investigate the impulse mitigation response. While Divinycell H130 foam-only samples could be manufactured by substituting the composite facesheet with a rigid aluminum facesheet, the hybrid corrugated cores could not easily be modified for this variation. As a result, each corrugated panel was manufacture with an additional rigid aluminum front facesheet attached to the original composite front facesheet, thereby leaving a laminated composite-aluminum front face. The panels were all tested at $14 \mathrm{~cm}$ standoff and 300 gram Detasheet explosive charge. The resulting impulse mitigation response, Figure 117, showed impulse mitigation $10-20 \%$ for samples that entered core crushing but did not reach core densification. The lower relative density samples reached full core densification and impulse increases up to $5 \%$ where observed. The rigid front facesheet samples exhibited a near identical 
impulse mitigation response to the composite facesheet cores at the impulse levels tested. The comparison of the impulse mitigation of all panels is shown in Figure 113.

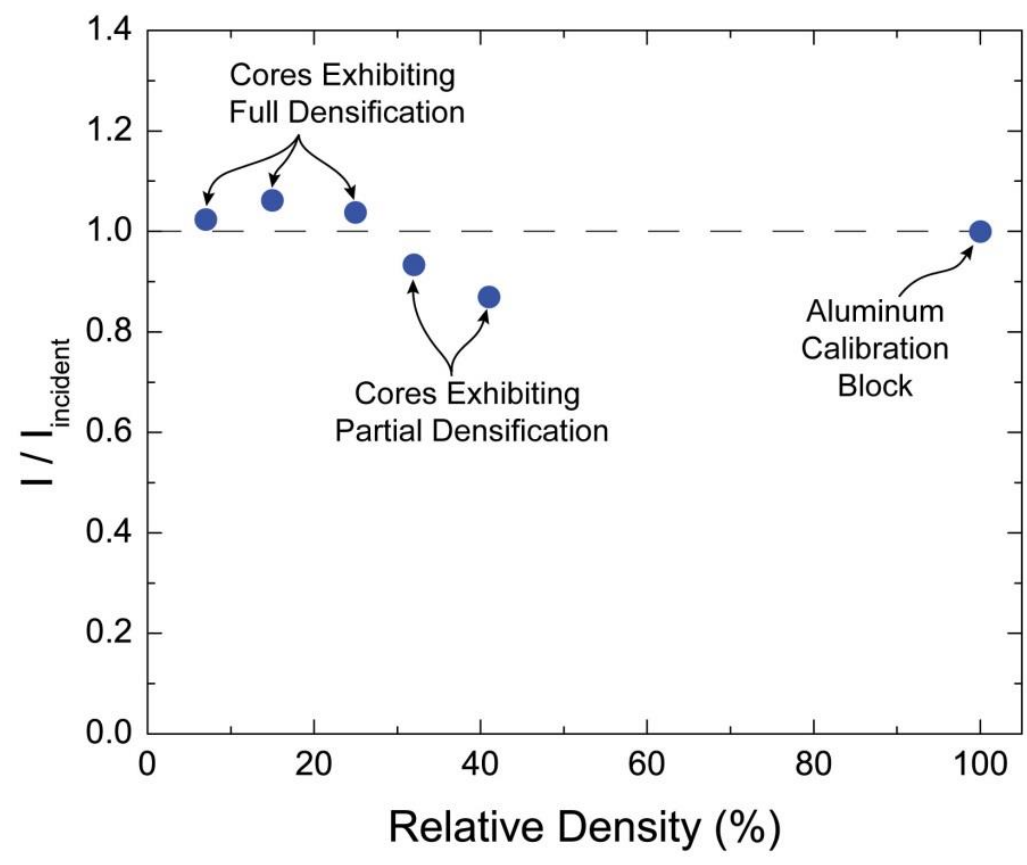

Figure 117: Normalized impulse measurement for the hybrid corrugated cores with rigid aluminum front facesheets added to minimize facesheet effects as measured by the vertical pendulum (sliding mode) and plotted against the relative density.

The hybrid corrugated sandwich panels show potential as a vehicle armor with a $30-40 \%$ through-structure pressure mitigation and up to a $30 \%$ impulse mitigation. The effect of a foamfilled core provides negligible mitigation for either pressure or impulse. Facesheet failure is predominant through all structures and may negate the advantages of the core by artificially increasing the transmitted impulse. Maintaining a rigid facesheet during impact can maintain the core as the predominate method of energy absorption. These results ultimately show that the hybrid corrugated sandwich panel is a potential solution to high explosive sand blast loading that would warrant the energies for future study. 


\section{Chapter 9}

\section{Research Contributions and Conclusions}

The focus of this research project was to investigate the mechanical response of a hybrid foam glass-epoxy composite corrugated sandwich panel structure under quasi-static and dynamic loading. This study chose to focus on only the rear-face supported boundary condition with the intent to assess the structure's mechanical behavior and viability for future analysis and development as underbelly vehicle armor. The research goals outlined for this study were the following:

- Identify and characterize the quasi-static response of this novel hybrid composite corrugated sandwich panel core along with the core struts and associated constitutive materials through empirical testing.

- Develop quasi-static analytic predictions for the strength and modulus of the core and core struts.

- Empirically investigate the dynamic strength and impulse mitigation performance along with the associated dynamic failure mechanisms.

Quasi-static, micromechanical models to predict the strength and stiffness for the 3D woven composite core strut and corrugated core were successfully developed and validated through 
empirical testing. Failure mechanisms for the core strut were identified along with the predominant variables that control strut strength and stiffness. These results will allow for future studies to optimize this (or similar) structures. This core was successfully tested under dynamic compressive loading and high explosive sand blast loading to investigate the high strain rate behavior, through-structure pressure reductions, and impulse mitigation. The successful measurements illuminate a regime of both pressure reduction and impulse mitigation for this panel under the loading levels and provide a basis to preliminary assess this structure's viability as vehicle armor.

\subsection{Contributions}

This research has been a multi-faceted project combining the design of the structure and testing methods, the development of a new manufacturing process, the micro-structure characterization of a complex 3D woven composite, and the mechanical testing of constitutive materials, composite struts, and hybrid structures under both quasi-static and dynamic loading. A broad scope of objectives has been defined and both significant and novel contributions have resulted from this study. The following is a highlight of the significant, novel, and useful contributions that I have made as a result of my efforts on this research project:

- Design of a hybrid foam glass-fiber composite corrugated cellular sandwich panel.

- Development of single-step, all-inclusive infusion and cure process of a dry fabric cellular structure sandwich panel with a pressure differential transfer technique to simultaneously remove air and resin vapor voids. 
- Adaptation and validation of unidirectional composite strength and stiffness (rule-ofmixture) models under tensile loading and the stiffness model under compressive loading for this $3 D$ woven fiber architecture.

- Development of new compressive strength model that accounts for the effect of fiber fraction and initial average fiber tow misalignment angles that is validated experimentally.

- Characterization of this 3D woven fiber architecture using optical microscopy and XCT imagery to investigate fiber orientation and misalignment.

- Determination of the compressive failure mechanisms associated with this $3 D$ woven architecture analysis including Euler buckling for slender struts, plastic microbuckling for thicker struts, and a plateau strength governed by fiber crushing for high strain rates. The transition position from Euler to plastic microbuckling was determined.

- Discovery that a localized maximum fiber tow misalignment angle does not control the maximum failure strength, rather an average of all axially loaded fiber tow misalignments control compressive failure. The initial average fiber tow misalignment is a predominant variable in the compressive strength.

- Discovery that the under compressive loading, the strength of the fiber material is not a critical design parameter. Higher strength (and higher cost) S2-glass fibers do not provide a benefit in compressive loading. 
- $\quad$ First time reporting of empirically determined mechanical behavior for this hybrid core under quasi-static loading, providing stress-strain responses, observed failure modes, peak strengths, stiffness, densification strain, and energy absorption, and comparison of the empirical results to established foam models.

- Development of densification strain model for the corrugated structure based on the development of hinge due initiated by unfractured fiber kinking within the composite.

- Co-design of novel vertical pendulum rig for rear-face blast loading that adapted for measuring impulse mitigation (sliding mode) and through-structure pressure mitigation (fixed/Kolsky Bar mode).

- Co-development of a novel flat sheet explosive blast assembly and characterization of the sand front velocity and position created by this charge technique.

- $\quad$ First time reporting of empirically determined impulse and through-structure pressure mitigation from high explosive sand blast loading on this hybrid corrugated structure and discovery of the associate dynamic failure modes.

- Discovery of an impulse mitigation regime and through-structure pressure capability that exist prior to core densification for this hybrid structure.

- Discovery that the thin, flexible facesheet can provide an increase in the transferred impulse and confirmation that a rigid front facesheet can significantly alter the response by altering the sand velocity and direction post impact. 


\subsection{Conclusion - Quasi-Static Response}

The hybrid foam glass-epoxy composite cellular structure sandwich panel is a novel structure that showed the capability to increase the critical compressive failure strength and energy over the Divinycell H130 foam-only and open core counterparts. The tensile and compressive moduli of the composite strut is well predicted with a rule-of-mixtures approach when approximating the system as a unidirectional laminate and accounting for initial average fiber tow misalignment. However, empirical knockdown parameters are required to quantitatively match the model with experimental results which is attributed to the fiber tow waviness created from the z-yarn impingement on neighboring fiber tows.

The out-of-plane compressive elastic modulus of the corrugated structures varied linearly with the composite corrugation volume fraction for volume fractions of 15 to $40 \%$, and was well predicted by a small-strain, straight-strut, pin-jointed model. The effect of the foam on the elastic modulus could be approximated with a rule-of-mixtures, and was small because the modulus of the foam was much less than that of the composite core. Further increases in elastic modulus of the core were more effectively achieved by increasing the fiber fraction in the strut loading direction or by substituting the mass of the foam core with additional composite material.

The tensile strength of the composite strut was successfully modeled using an iso-strain approach predicting failure by fiber yielding. This showed that in compression the dominant failure was observed by elastic (Euler) buckling for slender struts and plastic microbuckling for thicker struts. The Euler buckling prediction successful predicts the response of the slender struts, with strength being geometrically dependent, while the prediction of plastic microbuckling occurs through an iso-strain analysis of the transverse (warp) and axial (weft) 
fiber tows. The critical strength of the axially loaded tow laminates is approximated through a union of Argon's unidirectional fiber microbuckling prediction with a rule-of-mixtures approach to account for the effect of fiber fraction, while the transversely loaded tows laminates are based on the iso-stress assumption of fiber and matrix. The model provides a simplified approach to predicting the strength of a highly complex 3D woven composite with moderate accuracy. While not accounting for detailed fiber intricacies, variations in geometry, or void space, the model reveals that failure is predicted to primarily occur due to fiber volume fraction, matrix shear strength, and most importantly, the initial average fiber misalignment angle. Average tow misalignment through the thickness and width of a strut provide a representative value of overall initial average fiber misalignment. Good agreement exists between the model and experimental data on the $3 \mathrm{D}$ weave.

The compressive strength of the foam-filled composite core increased from 12 to $37 \mathrm{MPa}$ as the volume fraction of the corrugated material was increased from 15 to $40 \%$. The strength of both the empty and foam-filled structures exhibit an approximately linear dependence upon the volume fraction (or relative density) of the composite corrugation in agreement with predictions. The compressive strengths of the slenderest struts $(\mathrm{t} / \mathrm{l}=0.07)$ was controlled by Euler buckling, and otherwise by plastic microbuckling of the struts. Removal of the $3 \mathrm{MPa}$ plateau strength foam after fabrication of the composite reduced the strength of the core by 3-6 MPa consistent with a small synergistic influence of the foam linked to a delayed Euler buckling of the slenderest strut structure and local densification of the foam beyond its densification strain.

The micromechanical models successfully relate the corrugated structures modulus and strength to the geometry and properties of the composite materials. In general, good agreement was observed between the measurement, and the micromechanical predictions. The 
micromechanical model indicates that stronger, and more energy absorbing cellular structures could be fabricated by increasing the composite matrix shear strength, increasing the fiber volume fraction in the direction of loading, and reducing the misalignment angle of the fibers within the struts. During plastic microbuckling the fiber misalignment angle and the shear strength of the epoxy matrix controlled the strength. In this regime, the compressive strengths of these structures were approximately linearly dependent upon the strength of the struts, the relative density of the core and the strength of the foam. The use of z-yarns to increase resistance to delamination of the struts was the primary reason for the large misalignment angle in these materials.

Densification strain was insufficiently predicted by any known existing model. As a result, a new model was developed to reflect the observed behavior of the core based on empirical observations on the formation of a "hinge" within the core strut. Observations show that after initial microbuckling, fiber kinking continues to occur and propagate down the length of the strut resembling a structural hinge. Eventually, a geometric stability is reached signifying the densification strain, occurring after fractional strut length, $\mathrm{x}$, that has undergone kinking and the remainder of the undamaged strut can then withstand higher loads. Experimental measurements show that the hybrid structure densifies at a fractional strut length of $0.70 \pm 0.03$ of the total strut length, 1 . This value is experimentally observed to coincide with the strain at which continued compression occurs at a stress equal to the initial peak in strength. The mechanical hinge model predicts densification strains ranging from 53-62\% for open core structures and $38-44 \%$ for the foam-filled structures, in good agreement with experimental observations. 
The energy absorbed per volume during core compression to the onset of densification varied from 1.1 to $13 \mathrm{MJ} / \mathrm{m}^{3}$ as the relative density of the core (and its strength) was increased. The hybrid structure absorbed nearly 12 times the amount of energy as compared to the Divinycell H130 foam-only counterparts with an increase in density of only 6 times greater. The energy absorption of the hybrid structure was higher, on average, than that of the sum of the foam and empty core due to compression of the foam beyond its densification strain and stabilization of slender struts that failed by Euler (elastic) buckling. The hybrid foam corrugated panels showed a slight synergy at higher relative densities up to $3 \mathrm{MJ} / \mathrm{m}^{3}$ as compared with the open core counterparts.

Overall, while the use of higher strength S2 fibers increases the critical failure strength of 3D woven composites in tension, there is no evidence to the advantage of high strength fibers when used in compression. For this reason, the lower strength (lower cost) fibers are sufficient for strut manufacture in compressive applications.

\subsection{Conclusion - Dynamic Response}

Under dynamic loading, the deformation responses of the hybrid corrugated sandwich panels were reasonably uniform through the thickness of the core for impact velocities less than about $150 \mathrm{~ms}^{-1}$. At higher impact velocities deformation was observed localized near the impacted face, suggesting that the specimens were not in axial equilibrium. The dynamic compressive response of the hybrid corrugated sandwich panels showed the ability to provide a three to four times increase in the compressive strength of the core at high strain rates. An approximate linear relationship between compressive strength and strain rate was observed with a strength plateau reached at strain rates above $4000 \mathrm{~s}^{-1}$. Above this strain rate, fiber 
crushing/yielding of the glass fibers was observed and microbuckling was no longer the primary method of failure. The foam filling of the cores showed nearly no effect on the measured dynamic properties of the corrugated cores with the peak stresses (for either the composite strut or the corrugated cores). The increase in strength was attributed to the strain rate sensitivity of the composite matrix that stabilized the micro-buckling failure mode of the E-glass composite. It is anticipated that at higher applied strain rates than tested, no further increase in compressive strength would be observed and the glass-fiber crushing/yielding will remain the dominant failure mode.

The high-explosive sand blast loading on the three-tier variants of the hybrid corrugated sandwich structures provides an approximate linear sand front to evaluate the compressive loading response of the core. Sand velocities ranged from 100 to $400 \mathrm{~ms}^{-1}$ providing impulses (per area) ranging from 1 to $14 \mathrm{kPa}-\mathrm{sec}$. The vertical pendulum rig designed and developed for this study allowed for impulse mitigation (sliding mode) and rear-face pressure measurements (fixed mode) to be obtained. The hybrid cellular core could reduce the rear-face pressures by up to $40 \%$, as compared with a rigid, aluminum calibration block. Rig limitations restricted the full analysis of the higher strength structures to full densification. The response of Divinycell H130 foam only samples were investigated through regions of full densification and the effect of slap was observed which increases both the rear-face pressures and transmitted impulses to the structure. The hybrid structures were observed to reduce transmitted impulses by up to $30 \%$ if densification limits were not reached. The core was discovered to only be effective within a finite impulse range, acting as a rigid block at low applied impulse and exhibiting facesheet slap at higher impulses. 
Failure in the core was observed to occur by strut buckling, nodal rotations (which then initiated strut buckling), and strut shear. Failure by either Euler bucking could not be detected and failure by fiber crushing/yielding was not distinctly observed. The predominant failure method observed was facesheet failure. While this failure mode still provided validation of the overall structure performance, it could not solely provide core performance behavior. As a result, a rigid, aluminum facesheet was added to a select number of panels and further impulse mitigation ability was observed by the redirection of the soil ejecta in a lateral direction. Overall, this structure showed potential for impulse mitigation and pressure reductions under high explosive sand blast loading. 


\section{Chapter 10}

\section{Future Recommendation and Research Evaluation}

\subsection{Finite Element Modeling}

Advances in finite element analysis (FEA) over the past decade have significantly advanced the capabilities of modeling the dynamic behavior of a structure when subjected to a high explosive sand blast load. Historically, the computational requirements needed to model the high explosives, soil, air, and structural elements has limited the analysis of structural blast events to a select number of companies or government labs. Successful modeling capability is a valuable resource when investigating blast armor technologies. The huge costs associated with manufacturing and testing along with safety and regulation concerns when working with high explosives limit the capability for rigorous analysis for every designer, but especially research institutions and small companies.

Partnering with IMPETUS AFEA and CertaSIM LLC, a FEA model was developed using the IMEPETUS AFEA solver designed to investigate mine blast simulations. A unique particle method was developed by IMPETUS to compute the behavior of high explosives and the effects on soil, air, and structural elements. This code uses the parallel processing capabilities in advanced Graphics Processing Units (GPU) to run some of the most computationally difficult 
problems on a single desktop computer equipped with the proper technology. The modeling efforts released by IMPETUS and CertaSIM have consistently shown agreement with high explosive blast experiments. I pursued modeling efforts in this study, developing a corrugated sandwich structure to mirror the three-tier dynamic panels when subjected to high explosive sand blast loading with a rear-face supported boundary condition to predict the core response. While this FEA study is only in its infancy stage, the initial results have highlighted key failure mechanisms that were not originally considered primary failure mechanisms in the structures design. Analysis of a three-tier corrugated sandwich panel using the material properties of the S2-glass epoxy composite, used for the facesheets of the hybrid core, show facesheet failure as a primary failure mechanism. Further, strut buckling and nodal rotations are also predicted, particularly in a panel designed with nodal misalignments. Model validations of the IMPETUS AFEA code with researchers at UVA $[15,19,128]$ have shown an excellent ability to predict dynamic sand blast loading on cellular structures.

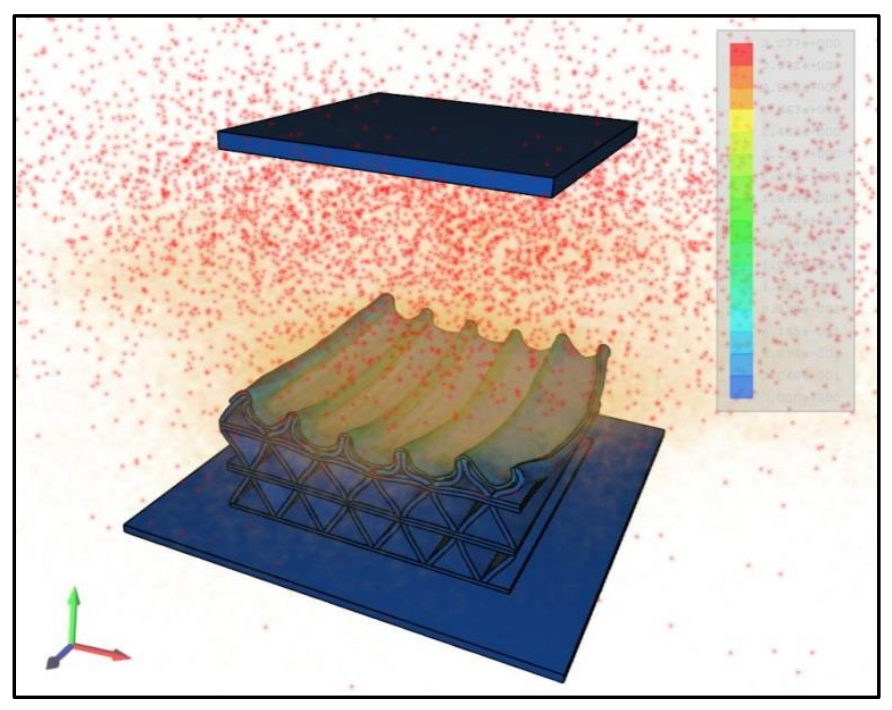

Figure 118: Advanced Finite Element Analysis (AFEA) can be used to predict the high explosive blast interaction with air, soil and structural elements. Primary failure mechanisms can be estimated before testing even begins. 
Future research routes with high explosive blast loading should evaluate the potential of using IMPETUS AFEA for design considerations, exploratory studies, detailed structural analysis and response, and model verification.

\subsection{Potential Improvements}

This research study on the hybrid foam glass-epoxy corrugated cellular structure sandwich panel has discovered many failure mechanism associated with the structure design and manufacture when subjected to compressive loading. Minimizing these imperfections will improve the structures peak strength capability and energy absorption ability. The primary improvement to improve the structures response and repeatability would be to maintain the core struts in precise alignment. The foam cores and hand manufacturing methods created struts with slight waviness from the beginning of manufacture. Further, the manufacture process applied sufficient pressures during infusion to further compress the structure and provide additional strut waviness while curing. Using rigid inserts during construction and a secure method to form and hold the facesheets in place during manufacture would improve the core strut considerably. Further, if the core struts could be placed in tension, Figure 119, during manufacture when folded over the rigid inserts, the struts could maintain a straighter alignment and increased fiber fractions, ideal for stretch-bending failure and increased peak strengths. Using an unbalanced 3D woven will further allow a larger fraction of the fibers to be oriented in the direction of axial compressive loading. Pre-tensioned fibers and unbalanced fabrics will allow for an increase in the fiber fraction and increase the compressive strength. Finally, increasing the matrix shear 
strength or using hybrid woven material, to incorporate the material benefits of multiple advanced fibers, are variations for potential improvement.

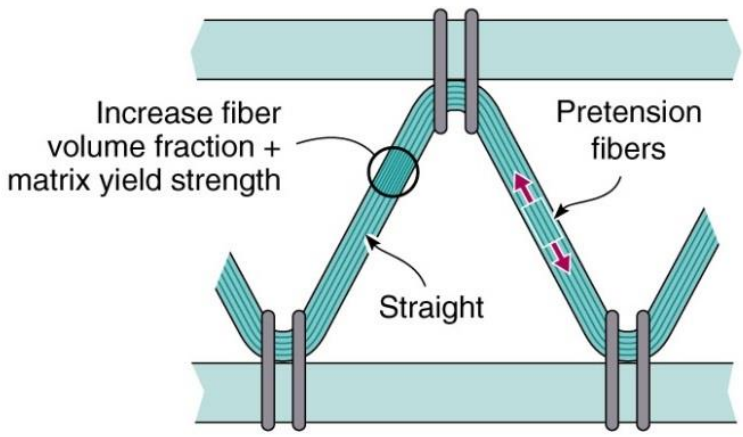

Figure 119: Pretension placed on the fibers could straighten the core struts and increase the fiber volume fraction, creating a much stronger corrugated cellular structure.

Energy absorption could easily be improved by the use of an alternate foam support. The development of extremely high compressive strength syntactic foams (foams that combine hollow microspheres with a matrix) provide a strong, lightweight option for the corrugated foam inserts. Increasing the strength of the foam within the cellular structure would provide a further stiffening effect on the strut. With sufficient foam strength, buckling failure could be delayed through strut stabilization, thereby providing synergism between the foam and strut.

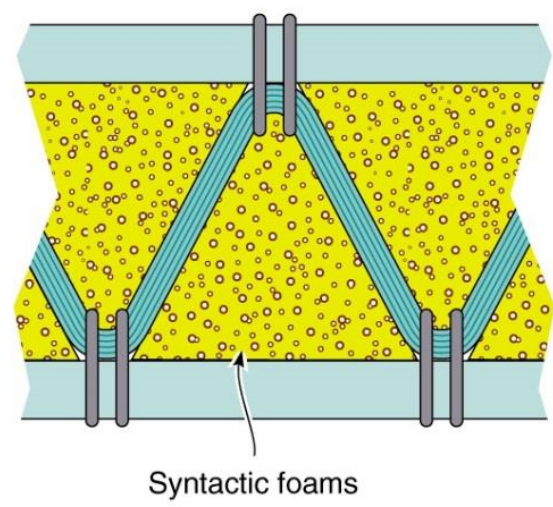

Figure 120: Syntactic foams used within the core could provide increased strength and energy absorption while maintaining a lightweight structure as well as provide a synergetic effect to strut buckling stabilization. 
Future designs should consider the adaptation of a multi-tier, multi-strength core. A core can be made sufficiently strong to survive a blast event, but if a structure does not deform it will not absorb energy and provide the needed use as an armor to mitigate impulse. If the structure is built to a smaller strength requirement, impulse mitigation will be successful at those smaller loading events, but catastrophic when a large event arises. The solution to consider would be a multi-tier, multi-strength core that would be adaptable to multiple levels of protection. Using a lower strength core as the first line of defense and increasing core strengths closer to the desired area of protection, a structure with sufficient strength is created to prohibit slap failure and maximize energy absorption through maximum panel deformation and damage.

\subsection{Overall Research Assessment}

The goal of this research was to design and evaluate a novel structure for potential use as a blast armor technology. A novel hybrid glass-epoxy corrugated cellular structure sandwich panel was designed to study the potential through-thickness pressure reduction and impulse mitigation, along with the overall quasi-static mechanical response. This development of this structure also created a new and unique method of manufacture, providing an all-inclusive, single-step infusion of the entire dry fabric sandwich panel and simultaneous resin vapor removal through a pressure differential translation technique. The dry fabric structure proved tedious to manufacture by hand, with unintentional imperfections created through the lack of manufacture consistency that is inherent to hand-built techniques. Difficulties in the analysis of performance data developed in this study due to an overwhelming number of variables created by the complexity of the structure and manufacture process. Regardless, the structures showed the capability to provide specific strengths comparable to steel while providing impulse 
mitigations up to $30 \%$ and through-structure pressure reductions up to $40 \%$. Undeniably, further design improvements in the fiber architecture, structure parameters, and manufacturing methods would be needed to further evaluate the successful use of this technology as an armor. The initial results from this study show the benefit of this structures potential use as a vehicle armor. The most promising feature of this structure is the refinement capability allowing the structure to be easily tailored for an alternate configuration during manufacture. As compared with metal cellular sandwich panels, this manufacturing process can be changed within minutes to create an alternate structure. Overall, this structure showed a finite impulse range in which successful impulse mitigation could occur. For real-world armor, a panel capable of significantly increased impulsive loads along with the range in size of potential threats would need to be developed. This hybrid structure shows the capability to be adapted to meet the demands for armor applications. 


\section{Reference}

[1] (2014). www.iCasualties.org/oef.

[2] A. B. Strong, Fundamentals of Composites Manufacturing: Materials, Methods, and Applications., 2 ed.: Society of Manufacturing Engineers, 2007.

[3] B. D. Craig, "High Performance Fibers for Lightweight Armor," AMPTIAC Quarterly, vol. 9, 2005 .

[4] B. A. Cheeseman and T. A. Bogetti, "Ballistic impact into fabric and compliant composite laminates," Composite Structures, vol. 61, pp. 161-173, 2003.

[5] R. B. Deo, J. H. J. Starnes, and Holzwarth, "Low-cost composite materials and structures for aircraft applications," presented at the RTO AVT Specialists Meeting on "Low Cost Composite Structures", Loen, Norway, 2001.

[6] S. D. L. M. U.S. Marine Corp. (2000). The USS Cole is towed away from the port city of Aden, Yemen. 001029-M-0557M-011

[7] D. I. D. s. member. (2011). Force Protection's MRAPs to Stalk Mines on the Battlefield.

[8] D. D. Radford, G. J. McShane, V. S. Deshpande, and N. A. Fleck, "The response of clamped sandwich plates with metallic foam cores to simulated blast loading," International Journal of Solids and Structures, vol. 43, pp. 2243-2259, 2006.

[9] K. P. Dharmasena, H. N. G. Wadley, Z. Y. Xue, and J. W. Hutchinson, "Mechanical response of metallic honeycomb sandwich panel structures to high-intensity dynamic loading," International Journal of Impact Engineering, vol. 35, pp. 1063-1074, Sep 2008.

[10] L. C. Taylor, R. R. Skaggs, and W. Gault, "Vertical impulse measurements of mines buried in saturated sand," Fragblast, vol. 9, pp. 19-28, 2005.

[11] F. Latourte, D. Grégoire, D. Zenkert, X. Wei, and H. D. Espinosa, "Failure mechanisms in composite panels subjected to underwater impulsive loads," Journal of the Mechanics and Physics of Solids, vol. 59, pp. 1623-1646, 2011.

[12] D. Zenkert, A. Shipsha, P. Bull, and B. Hayman, "Damage tolerance assessment of composite sandwich panels with localised damage," Composites Science and Technology, vol. 65, pp. 2597 2611, 2005.

[13] K. P. Dharmasena, D. T. Queheillalt, H. N. G. Wadley, P. Dudt, Y. Chen, D. Knight, et al., "Dynamic compression of metallic sandwich structures during planar impulsive loading in water," European Journal of Mechanics a-Solids, vol. 29, pp. 56-67, Jan-Feb 2010. 
[14] K. P. Dharmasena, H. N. G. Wadley, K. Williams, Z. Xue, and J. W. Hutchinson, "Response of metallic pyramidal lattice core sandwich panels to high intensity impulsive loading in air," International Journal of Impact Engineering, vol. 38, pp. 275-289, 2011.

[15] J. J. Rimoli, B. Talamini, J. J. Wetzel, K. P. Dharmasena, R. Radovitzky, and H. N. G. Wadley, "Wet-sand impulse loading of metallic plates and corrugated core sandwich panels," International Journal of Impact Engineering, vol. 38, pp. 837-848, 2011.

[16] D. Zenkert, The Handbook of Sandwich Construction. Worcestershire, United Kingdom: EMAS Publishing, 1997.

[17] M. F. Ashby, "The properties of foams and lattices," Philos Trans A Math Phys Eng Sci, vol. 364, pp. 15-30, 2006.

[18] T. Liu, N. A. Fleck, H. N. G. Wadley, and V. S. Deshpande, "The impact of sand slugs against beams and plates: Coupled discrete particle/finite element simulations," Journal of the Mechanics and Physics of Solids, vol. 61, pp. 1798-1821, 2013.

[19] H. N. G. Wadley, T. Børvik, L. Olovsson, J. J. Wetzel, K. P. Dharmasena, O. S. Hopperstad, et al., "Deformation and fracture of impulsively loaded sandwich panels," Journal of the Mechanics and Physics of Solids, vol. 61, pp. 674-699, 2013.

[20] K. P. Dharmasena, H. N. G. Wadley, T. Liu, and V. S. Deshpande, "The dynamic response of edge clamped plates loaded by spherically expanding sand shells," International Journal of Impact Engineering, vol. 62, pp. 182-195, 2013.

[21] M. F. Ashby, Materials Selection in Mechanical Design, 4th ed. Elsevier: ButterworthHeinemann, 2011.

[22] B. D. Agarwal, L. J. Broutman, and K. Chandrashekhara, Analysis and Performance of Fiber Composites, 3 ed.: John Wiley \& Sons, 2006.

[23] Greene, Marine Composites, 2 ed. Annapolis, Maryland: Eric Greene Associates, Inc., 1999.

[24] A. Miravete, 3-D textile reinforcements in composite materials: CRC Press, 1999.

[25] A. Mortensen, Concise Encyclopedia of Composite Materials, 2 ed.: Elsevier, 2007.

[26] L. J. Gibson and M. F. Ashby, Cellular Solids: Structures and Properties, 2 ed.: Cambridge University Press, 1999.

[27] M. F. Ashby, A. G. Evan, N. A. Fleck, L. J. Gibson, J. W. Hutchison, and H. N. G. Wadley, Metal foams: a design guide. Boston: Butterworth Heinemann, 2000.

[28] P. Moongkhamklang, "Titanium-Silicon Carbide Composite Lattice Structures."

[29] N. Bourne, Materials in Mechanical Extremes: Cambridge University Press, 2013.

[30] L. J. Gibson, M. F. Ashby, and B. A. Harley, Cellular Materials in Nature and Medicine: Cambridge University Press, 2010. 
[31] N. Mills, Polymer Foams Handbook: Engineering and Biomechanics Applications and Design Guide: Butterworth-Heinemann, 2007.

[32] H. N. G. Wadley, "Cellular Metals Manufacturing," Advanced Engineering Materials, vol. 4, pp. 726-733, 2002.

[33] J. Banhart, J. Baumeister, and M. Weber, "Damping Properties of Aluminum Foams," Materials Science and Engineering, vol. A205, pp. 221-228, 1996.

[34] D. T. Queheillalt, B. W. Choi, D. S. Schwartz, and H. N. G. Wadley, "Creep Expansion of Porus Ti-6Al-4V Sandwich Structures," Metallurgical and Materials Transactions A, vol. 31A, pp. 261$273,2000$.

[35] D. M. Elzey and H. N. G. Wadley, "The Limits of Solid State Foaming," Acta Materialia, vol. 49, pp. 849-859, 2001.

[36] M. Scheffler, P. Colombo, and J. Binner, Cellular Ceramics: Structure, Manufacturing, Properties, and Applications, Chapter 2.1: Ceramics Foams: Wiley-VCH, 2005.

[37] B. P. Russell, A. J. Malcom, H. N. G. Wadley, and V. S. Deshpande, "Dynamic Compressive Response of Composite Corrugated Cores," Journal of Mechanics of Materials and Structures, vol. 5, pp. 477-493, 2010.

[38] M. F. Ashby, A. G. Evans, N. A. Fleck, L. J. Gibson, J. W. Hutchinson, and H. N. G. Wadley, Metal Foams: A Design Guide. Boston: Butterworth \& Heinemann, 2000.

[39] K. W. Suh, C. P. Park, M. J. Maurer, M. H. Tusim, R. D. Genova, R. Broos, et al., "Lightweight Cellular Plastics," Advanced Materials, vol. 12, pp. 1779-1789, December 12000.

[40] M. Scheffler and P. Colombo, Eds., Cellular Ceramics: Structure, Manufacturing, Properties and Applications. John Wiley and Sons Inc, 2005, p.^pp. Pages.

[41] J. Lim and K. Kang, "Mechanical behavior of sandwich panels with tetrahedral and Kagome truss cores fabricated from wires," International Journal of Solids and Structures vol. 43, pp. 52285246, 2006.

[42] D. T. Queheillalt and H. N. G. Wadley, "Pyramidal lattice truss structures with hollow trusses," Materials Science and Engineering A vol. 397 pp. 132-137, 2005.

[43] G. W. Kooistra and H. N. G. Wadley, "Lattice truss structures from expanded metal sheet," Materials and Design vol. 28, pp. 507-514, 2007.

[44] D. T. Queheillalt and H. N. G. Wadley, "Titanium alloy pyramidal lattice truss structures," Materials and Design, In press.

[45] J. Wang, A. G. Evans, K. Dharmasena, and H. N. G. Wadley, "On the performance of truss panels with Kagomé cores," International Journal of Solids and Structures, vol. 40, pp. 69816988, 2003. 
[46] D. J. Sypeck and H. N. G. Wadley, "Multifunctional microtruss laminates: textile synthesis and properties," Journal of Materials Research, vol. 16, pp. 890-897, 2001.

[47] J. Tian, T. Kim, T. J. Lu, H. P. Hodson, D. T. Queheillalt, and H. N. G. Wadley, "The effects of topology upon fluid-flow and heat-transfer within cellular copper structures," International Journal of Heat and Mass Transfer, vol. 47 pp. 3171-3186, 2004.

[48] M. Zupan, V. S. Deshpande, and N. A. Fleck, "The out-of-plane compressive behavior of wovencore sandwich plates," European Journal of Mechanics A- Solids, vol. 23, pp. 411-421, 2004.

[49] D. T. Queheillalt and H. N. G. Wadley, "Cellular metal lattices with hollow trusses," Acta Materialia vol. 53, pp. 303-313, 2005.

[50] V. S. Deshpande and N. A. Fleck, "Collapse of truss core sandwich beams in 3-point bending," International Journal of Solids and Structures, vol. 38, pp. 6275-6305, 2001.

[51] S. Chiras, D. R. Mumm, A. G. Evans, N. Wicks, J. W. Hutchinson, K. Dharmasena, et al., "The Structural performance of near-optimized truss core panels " International Journal of Solids and Structures, vol. 39, pp. 4093-4115, 2002.

[52] H. N. G. Wadley, N. A. Fleck, and A. G. Evans, "Fabrication and structural performance of periodic cellular metal sandwich structures " Composites Science and Technology, vol. 63, pp. 2331-2343, 2003.

[53] G. W. Kooistra, V. S. Deshpande, and H. N. G. Wadley, "Compressive behavior of age hardenable tetrahedral lattice truss structures made from aluminium," Acta Matertialia, vol. 52, pp. 4229-4237, 2004.

[54] G. W. Kooistra and H. N. G. Wadley, "Lattice truss structures from expanded metal sheet," Materials \& Design, vol. 28, pp. 507-514, 2007.

[55] K. Finnegan, G. Kooistra, H. N. G. Wadley, and V. S. Deshpande, "The Compressive Response of Carbon Fiber Composite Pyramidal Truss Sandwich Cores," International Journal of Materials Research, vol. 98, pp. 1-9, 2007.

[56] B. P. Russell, V. S. Deshpande, and H. N. G. Wadley, "Quasistatic deformation and failure modes of composite square honeycombs," Journal of Mechanics of Materials and Structures, vol. 3, pp. 1315-1335, 2008.

[57] T. George and H. Wadley, "Shear behavior of carbon fiber composite pyramidal truss sandwich cores," In preparation.

[58] S. K. Maiti, L. J. Gibson, and M. F. Ashby, "Deformation and Energy Absorption Diagrams for Cellular Solids," Acta Materialia, vol. 32, pp. 1963-1975, 1984.

[59] H. N. G. Wadley, "Multifunctional periodic cellular metals," Phil. Trans. Royal Society, vol. 364, pp. 31-68, Jan 152006. 
[60] H. G. Allen, "Analysis and design of structural sandwich panels," ed Oxford: Pergamon Press, 1969.

[61] D. Zenkert, An introduction to sandwich construction. London: Engineering Materials Advisory Service Ltd., 1995.

[62] AVStop.com. (2014, July 1). Laminated Structures.

[63] T. Bitzer, Honeycomb technology: materials, design, manufacturing, applications and testing: Chapman \& Hall, 1997.

[64] C. C. Foo, G. B. Chai, and L. K. Seah, "Mechanical properties of Nomex material and Nomex honeycomb structure," Composite Structures, vol. 80, pp. 588-594, 2007.

[65] M. Kintscher, L. Kärger, A. Wetzel, and D. Hartung, "Stiffness and failure behaviour of folded sandwich cores under combined transverse shear and compression," Composites part A: Applied science and manufacturing vol. 38, pp. 1288-1295, 2007.

[66] W.-Y. Jung and A. J. Aref, "A combined honeycomb and solid viscoelastic material for structural damping applications," Mechanics of Materials vol. 35, pp. 831-844, 2003.

[67] J. K. Paik, A. K. Thayamballi, and G. S. Kim, "The strength characteristics of aluminum honeycomb sandwich panels," Thin-Walled Structures vol. 35, pp. 205-231, 1999.

[68] M. Doyoyo and D. Mohr, "Microstructural response of aluminum honeycomb to combined outof-plane loading," Mechanics of Materials vol. 35, pp. 865-876, 2003.

[69] H. Wenbo, Z. Kaifeng, and W. Guofeng, "Superplastic forming and diffusion bonding for honeycomb structure of Ti-6Al-4V alloy," Journal of Materials Processing Technology vol. 183, pp. 450-454, 2007.

[70] J. M. Williams and R. G. Kelly, "An analysis of corrosive species ingress into IM7/PETI-5 Ti honeycomb composites," Composites Science and Technology vol. 64, pp. 1875-1883, 2004.

[71] F. Côté, V. S. Deshpande, N. A. Fleck, and A. G. Evans, "The compressive and shear responses of corrugated and diamond lattice materials," International Journal of Solids and Structures vol. 43, pp. 6220-6242, 2006.

[72] F. W. Zok, H. J. Rathbun, Z. Wei, and A. G. Evans, "Design of metallic textile core sandwich panels," International Journal of Solids and Structures, vol. 40, pp. 5707-5722, 2003.

[73] A. J. Malcom, M. T. Aronson, V. S. Deshpande, and H. N. G. Wadley, "Compressive Response of Glass Fiber Composite Sandwich Structures," Composites Part A: Applied Science and Manufacturing, vol. 54, pp. 88-97, 2013.

[74] N. Wicks and J. W. Hutchinson, "Opitmal truss plates," International Journal of Solids and Structures, vol. 38, pp. 5165-5183, 2001. 
[75] J. C. Wallach and L. J. Gibson, "Mechanical behavior of a three-dimensional truss material," International Journal of Solids and Structures, vol. 38, pp. 7181-7196, 2001.

[76] T. J. Lu, H. A. Stone, and M. F. Ashby, "Heat transfer in open-cell metal foams," Acta Materialia vol. 46, pp. 3619-3635, 1998.

[77] K. Boomsma and D. Poulikakos, "On the effective thermal conductivity of a three-dimensionally structured fluid-saturated metal foam," International Journal of Heat and Mass Transfer vol. 44, pp. 827-836, 2001.

[78] K.-H. Ko and N. K. Anand, "Use of porous baffles to enhance heat transfer in a rectangular channel," International Journal of heat and mass transfer, vol. 46, pp. 4191-4199, 2003.

[79] V. S. Deshpande and N. A. Fleck, "High strain rate compressive behavior of aluminium alloy foams," International Journal of Impact Engineering, vol. 24, pp. 277-298, 2000.

[80] I. W. Hall, M. Guden, and C.-J. Yu, "Crushing of aluminum closed cell foams: density and strain rate effects," Scripta Materialia vol. 43, pp. 515-521, 2000.

[81] A. Paul and U. Ramamurty, "Strain rate sensitivity of a closed-cell aluminum foam," Materials Science and Engineering: A, vol. 281, pp. 1-7, 2000.

[82] T. J. Lu, A. Hess, and M. F. Ashby, "Sound absorption in metallic foams," Journal of Applied Physics, vol. 85, pp. 7528-7539, 1999.

[83] X. Wang and T. J. Lu, "Optimized acoustic properties of cellular solids," Journal of the Acoustical Society of America, vol. 106, pp. 756-765, 1999.

[84] W. Jiejun, L. Chenggong, W. Dianbin, and G. Manchang, "Damping and sound absorption properties of particle reinforced Al matrix composite foams," Composites Science and Technology, vol. 63, pp. 569-574, 2003.

[85] A. G. Hanssen, "Close-range blast loading of aluminum foam panels."

[86] D. D. Radford, G. J. McShane, V. S. Deshpande, and N. A. Fleck, "Dynamic Compressive Response of Stainless-Steel Square Honeycombs," Journal of Applied Mechanics, vol. 74, p. 658, 2007.

[87] V. Rubino, V. S. Deshpande, and N. A. Fleck, "The dynamic response of end-clamped sandwich beams with a Y-frame or corrugated core," International Journal of Impact Engineering, vol. 35, pp. 829-844, 2008.

[88] V. L. Tagarielli, V. S. Deshpande, and N. A. Fleck, "Prediction of the dynamic response of composite sandwich beams under shock loading," International Journal of Impact Engineering, vol. 37, pp. 854-864, 2010. 
[89] H. Wadley, K. Dharmasena, Y. Chen, P. Dudt, D. Knight, R. Charette, et al., "Compressive response of multilayered pyramidal lattices during underwater shock loading," International Journal of Impact Engineering, vol. 35, pp. 1102-1114, 2008.

[90] R. R. Skaggs, W. N. Roy, B. A. Cheeseman, Y. Huang, B. Pandurangan, and M. Grujicic, "Impulse loading resulting from shallow buried explosives in water-saturated sand," Proceedings of the Institution of Mechanical Engineers, Part L: Journal of Materials: Design and Applications, vol. 221, pp. 21-35, 2007.

[91] A. Vaziri, Z. Xue, and J. W. Hutchinson, "Performance and failure of metal sandwich plates subjected to shock loading," Journal of Mechanics of Materials and Structures, vol. 2, 2007.

[92] H. J. Rathbun, Z. Wei, M. Y. He, F. W. Zok, A. G. Evans, D. J. Sypeck, et al., "Measurement and Simulation of the Performance of a Lightweight Metallic Sandwich Structure With a Tetrahedral Truss Core," Journal of Applied Mechanics, vol. 71, p. 368, 2004.

[93] G. Kooistra, V. S. Deshpande, and H. N. G. Wadley, "Compressive behavior of age hardenable tetrahedral lattice truss structures made from aluminium," Acta Materialia, vol. 52, pp. 42294237, 2004.

[94] N. J. Mills and A. Gilchrist, "The Effectiveness of Foams in Bicycle and Motorcycle Helments," Accident Analysis and Prevention, vol. 23, pp. 153-163, 1991.

[95] Z. Wei, K. P. Dharmasena, H. N. G. Wadley, and A. G. Evans, "Analysis and interpretation of a test for characterizing the response of sandwich panels to water blast," International Journal of Impact Engineering, vol. 34, pp. 1602-1618, 2007.

[96] H. N. G. Wadley, K. P. Dharmasena, M. Y. He, R. M. McMeeking, A. G. Evans, T. Bui-Thanh, et al., "An active concept for limiting injuries caused by air blasts," International Journal of Impact Engineering, vol. 37, pp. 317-323, 2010.

[97] T. Børvik, L. Olovsson, A. G. Hanssen, K. P. Dharmasena, H. Hansson, and H. N. G. Wadley, "A discrete particle approach to simulate the combined effect of blast and sand impact loading of steel plates," Journal of the Mechanics and Physics of Solids, vol. 59, pp. 940-958, 2011.

[98] J. LeBlanc, A. Shukla, C. Rousseau, and A. Bogdanovich, "Shock loading of three-dimensional woven composite materials," Composite Structures, vol. 79, pp. 344-355, 2007.

[99] J. Grogan, S. A. Tekalur, A. Shukla, A. Bogdanovich, and R. A. Coffelt, "Ballistic Resistance of 2D and 3D Woven Sandwich Composites," Journal of Sandwich Structures and Materials, vol. 9, pp. 283-302, 2007.

[100] B. P. Russell, V. S. Deshpande, and H. N. G. Wadley, "Quasi-static Deformation and Failure Modes of Composite Square Honeycombs," Journal of Mechanics of Materials and Structures, vol. 3, pp. 1315-1339, 2008. 
[101] Granta Design Limited CES EduPack 2011 [Online].

[102] DuPont. (2014, 07-01-2014). Kevlar Brand Overview - Website http://www.dupont.com/products-and-services/fabrics-fibersnonwovens/fibers/brands/kevlar.html.

[103] DuPont, "Technical Guide - Kevlar - Aramid Fiber," in DuPont Material Guide Publication vol. H-77848 4/00, ed, 2000.

[104] A. Poleramic. (2007, 07-02-2014). Applied Poleramic - VARTM Resin/RTM Resin - Web Site http://www.appliedpoleramic.com/index.php?q=infusion_epoxy.html.

[105] D. Group, "DIAB - Divinycell H Series Techincal Data Pamphlet," vol. H.ds-u/04_2007.rev1, DIAB, Ed., ed, 2007.

[106] C. R. Calladine and R. W. English, "Strain-rate and inertia effects in the collapse of two types of energy-absorbing structure " International Journal of Mechanical Sciences, vol. 26, pp. 689-701, 1984.

[107] L. Tong, A. P. Mouritz, and M. K. Bannister, 3D Fibre Reinforced Polymer Composites. Oxford: Elsevier, 2002.

[108] K. Pochiraju and T.-W. Chou, "Three-Dimensionally Woven and Braided Composites. II: An Experimental Characterization," Polymer Composites, vol. 20, pp. 733-747, 1999.

[109] B. N. Cox, M. S. Dadkhah, W. L. Morris, and J. G. Flintoff, "Failure Mechanisms of 3D Woven Composites in Tension, Compression, and Bending," Acta metall. mater, vol. 42, pp. 3967-3984, 1994.

[110] B. N. Cox, M. S. Dadkhah, and W. L. Morris, "On the Tensile Failure of 3D Woven Composites," Composites Part A, vol. 27A, pp. 447-458, 1996.

[111] K. K. Chawla, Fibrous Materials: Cambridge Unviersity Press, 1998.

[112] C. R. Calladine, "Understanding Imperfection-Sensitivity in the Buckling of Thin-Walled Shells," Thin-Walled Structures, vol. 23, pp. 215-235, 1995.

[113] J. Xiong, L. Ma, L. Wu, B. Wang, and A. Vaziri, "Fabrication and Crushing Behavior of Low Density Carbon Fiber Composite Pyramidal Truss Structures," Composite Structures, vol. 92, pp. 2695-2702, 2010.

[114] N. A. Fleck, "Compressive Failure of Fiber Composites," Advances in Applied Mechanics, vol. 33, pp. 43-119, 1997.

[115] L. Bartosiewics and Z. Mencik, "An Etching Technique to Reveal the Supermolecular Structure of Crystalline Polymers," Journal of Polymer Science, vol. 12, pp. 1163-1175, 1974.

[116] L. C. Sawyer and D. T. Grubb, Polymer Microscopy, 2 ed. London: Chapman \& Hall, 1996.

[117] A. K. Kaw, Mechanics of Composite Materials: CRC Press, 1997. 
[118] A. S. Argon, Fracture of Composites vol. 1. New York: Academic Press, 1972.

[119] N. A. Fleck, P. M. Jelf, and P. T. Curtis, "Compressive Failure of Laminated and Woven Composites," Journal of Composites Technology \& Research, vol. 17, pp. 212-220, 1995.

[120] B. Budiansky and N. A. Fleck, "Compressive Failure of Fibre Composites," Journal of Mechanics and Physics of Solids, vol. 41, pp. 183-211, 1993.

[121] B. Budiansky and N. A. Fleck, "Compressive kinking of Fiber composites: A Topical Review," Appl Mech Rev, vol. 47, 1994.

[122] T. George, "Private Communication: Quasi-Static Testing of Divinycell Foam Materials: Unpublished Research Material," in University of Virginia, A. J. Malcom, Ed., ed, 2012.

[123] K. Dharmasena, D. Queheillalt, H. Wadley, Y. Chen, P. Dudt, D. Knight, et al., "Dynamic response of a multilayer prismatic structure to impulsive loads incident from water," International Journal of Impact Engineering, vol. 36, pp. 632-643, Apr 2009.

[124] W. Chen and B. Song, Split Hopkinson (Kolsky) Bar: Design, Testing, and Applications: Springer, 2011.

[125] J. Fan and W. S. Slaughter, "High strain rate compression of fiber composites," Journal of Mechanics and Physics of Solids, vol. 45, pp. 731-751, 1997.

[126] M. T. Tilbrook, D. D. Radford, V. S. Deshpande, and N. A. Fleck, "Dynamic crushing of sandwich panels with prismatic lattice cores," International Journal of Solids and Structures, vol. 44, pp. 6101-6123, 2007.

[127] J. Banhart, "Manufacture, characterisation and application of cellular metals and metal foams," Progress in Materials Science, vol. 46, pp. 559-632, 2001.

[128] R. L. Holloman, "Impulse Loading of 3D Prismatic Cellular Structures," Doctor of Philosophy, Materials Science and Engineering, University of Virginia, 2014.

[129] G. J. McShane, V. S. Deshpande, and N. A. Fleck, "The underwater blast resistance of metallic sandwich beams with prismatic lattice cores," Journal of Applied Mechanics-Transactions of the Asme, vol. 74, pp. 352-364, Mar 2007.

[130] H. N. G. Wadley, K. P. Dharmasena, M. R. O'Masta, and J. J. Wetzel, "Impact response of aluminum corrugated core sandwich panels," International Journal of Impact Engineering, vol. 62, pp. 114-128, 2013.

[131] Z. Wei, V. S. Deshpande, A. G. Evans, K. P. Dharmasena, D. T. Queheillalt, H. N. G. Wadley, et al., "The resistance of metallic plates to localized impulse," Journal of the Mechanics and Physics of Solids, vol. 56, pp. 2074-2091, 2008. 
[132] L. F. Mori, S. Lee, Z. Y. Xue, A. Vaziri, D. T. Queheillalt, K. P. Dharmasena, et al., "Deformation and Fracture Modes of Sandwich Structures Subjected to Underwater Impulsive Loads," Journal of Mechanics of Materials and Structures, vol. 2, pp. 1981-2006, 2007.

[133] W. L. Fourney, U. Leiste, R. Bonenberger, and D. Goodings, "Explosive impulse on plates," Fragblast, vol. 9, pp. 1-17, 2005.

[134] T. Liu, H. N. G. Wadley, and V. S. Deshpande, "Dynamic compression of foam supported plates impacted by high velocity soil," International Journal of Impact Engineering, vol. 63, pp. 88-105, 2014. 